\title{
The QUEST Questionnaire System
}

Kelvin Morton BE (Hons)

A thesis submitted in partial fulfilment

of the requirements for the degree of

Master of Engineering

in

Electrical and Electronic Engineering

at the

University of Canterbury,

Christchurch, New Zealand.

25 June 1996 



\begin{abstract}
The thesis looks at the implementation of a computer-based questionnaire system, QUEST. The motivation to develop such a system arose from the lack of lifestyle questionnaires for general practitioners to use in health screening. In the course of producing a lifestyle questionnaire, the process of gathering information by questionnaire or survey was examined. From this a set of requirements for a computer-based questionnaire system were developed and two questionnaire models to meet these requirements were designed.

The Question Order flow model and the Required Information flow model are presented and their advantages and disadvantages are discussed. The Question Order flow model proved to be an unreliable way of representing a questionnaire as it focused only on question ordering within the questionnaire structure. The Required Information flow model was developed to overcome the main deficiencies of Question Order flow model by focusing on the information relationships within the questionnaire structure.

The strengths and weaknesses of the implemented questionnaire systems are discussed. Several actual paper questionnaires implemented with QUEST are then presented. From this a set of future recommendations for QUEST are made based on the feedback of users of the system.
\end{abstract}





\section{ACKNOWLEDGEMENTS}

There are a number of people I wish to thank for their help during the preparation of this thesis and the work which preceded it. Firstly I would like to thank Dr Chris Carey-Smith for his input, patience and guidance over the last three years. I also thank Dr Keith Carey-Smith for his enthusiasm in starting this project and in continuing with the development and testing aspects of producing software that works, Tait Electronics for their contribution towards the development of this project in the first year of my degree, Geoff Scarr for his support and understanding during a difficult time, Dr Phil Bones for getting me over the final hurdle and being so easy to work with. I would like to thank my father for making this thesis firstly possible and then finally a reality. I will miss you. Finally I would like to thank my family and friends for ensuring that you are now reading this thesis (a little late maybe, but that's fine by me).

"Of course, it is very important to be sober when you take an exam. Many worthwhile careers in the street-cleansing, fruit-picking and subway-guitarplaying industries have been founded on a lack of understanding of this simple fact." - (Terry Pratchett, Moving Pictures)

"The only thing known to go faster than ordinary light is monarchy, according to the philosopher Ly Tin Weedle. He reasoned like this: you can't have more than one king, and tradition demands that there is no gap between kings, so when a king dies the succession must therefore pass to the heir instantaneously. Presumably, he said, there must be some elementary particles - kingons, or possibly queons - that do this job, but of course succession sometimes fails if, in mid-flight, they strike an anti-particle, or republicon. His ambitious plans to use his discovery to send messages, involving the careful torturing of a small king in order to modulate the signal, were never fully expanded because, at that point, the bar closed." - (Terry Pratchett, Mort)

"Ankh-Morpork had dallied with many forms of government and had ended up with that form of democracy known as One Man, One Vote. The Patrician was the Man; he had the Vote." - Discworld politics explained (Terry Pratchett, Mort) 



\section{CONTENTS}

$\begin{array}{ll}\text { ABSTRACT } & \text { iii }\end{array}$

ACKNOWLEDGEMENTS

CHAPTER 1 INTRODUCTION 1

CHAPTER 2 RELATED FIELDS OF RESEARCH 5

2.1 Questionnaire and Survey Design 5

2.1.1 Questionnaire Types 8

2.1.2 Quality of Information 13

2.1.3 Question Types 15

2.2 Expert Systems 19

2.2.1 Motivations for making an Expert System 20

2.2.2 Expert System Structure $\quad 22$

2.2.3 Knowledge Representation 23

2.2.4 Inference Techniques 25

CHAPTER 3 QUEST DESIGN AND MODELING 35

3.1 The Questionnaire Process 35

3.2 Computer Based Questionnaire Requirements 38

3.3 Question Order Flow Approach 42

3.3.1 The Questionnaire Structure Model 43

3.3.2 Implications for the Author 50

3.3.3 Implications for the Respondent 52

3.3.4 Implementations $\quad 53$

3.3.5 Summary 60

3.4 Required Information Flow Approach 62

3.4.1 The Questionnaire Structure Model . 63

3.4.2 Implications for the Author 66

3.4.3 Implications for the Respondent 67

3.4.4 Implementation $\quad 67$

$\begin{array}{lll}\text { CHAPTER } 4 & \text { QUESTEX IMPLEMENTATION } & 69\end{array}$

4.1 Initialisation $\quad 70$

4.2 Database Management $\quad 73$ 
4.3 Inference Engine $\quad 76$

4.3.1 The ENG_SubEngine Predicate $\quad 76$

4.3.2 Back and Quit Functions $\quad 80$

4.4 Calculations and Conditions $\quad 82$

4.5 User Interface $\quad 86$

4.5.1 Template Manager $\quad 86$

4.5.2 Template Initialisation $\quad 90$

4.5.3 Template Rendering $\quad 90$

4.5.4 Queuing Multimedia Events 93

4.5.5 Display Template $\quad 96$

4.5.6 Closing the Template $\quad 97$

4.6 Questionnaire Summaries $\quad 99$

4.6.1 Summary Processing 100

4.6.2 Summary Generation 101

4.6.3 Summary Printing 102

4.7 Summary 104

$\begin{array}{lll}\text { CHAPTER } 5 & \text { QUESTED IMPLEMENTATION } & 105\end{array}$

5.1 Initialisation 106

5.2 Database Management _ 107

5.3 Questionnaire Editor 109

$\begin{array}{ll}\text { 5.3.1 Tree View } & 109\end{array}$

5.3.2 Property Panels 112

5.3.3 Questionnaire operations 116

5.3.4 Calculations and Conditions 119

5.4 Template Editor $\quad 120$

5.4.1 Template Manager 121

5.4.2 Creating New Templates 122

5.4.3 Template Editor View 124

5.4.3.1 Object View 124

5.4.3.2 Object Selection $\quad 127$

5.4.3.3 Object Operations 127

5.4.3.4 Object Properties 130

5.5 Summary Editor 132

5.5.1 Entering a Summary 132

5.5.2 Summary Entries 134

5.6 QuestView Implementation 135

5.7 Summary 136

$\begin{array}{lll}\text { CHAPTER } 6 & \text { PRELIMINARY EXPERIENCE } & 139\end{array}$

6.1 Paper Questionnaire Implementations 139

$\begin{array}{lll}6.1 .1 & \text { LifeQuest } & 139\end{array}$

6.1.2 Stroke Questionnaire 140

6.1.3 Smoking Questionnaires 140

6.1.4 Readiness to Change Questionnaire 141 
6.1.5 AUDIT Questionnaire 141

6.1.6 HealthCheck Staff Questionnaire 141

6.1.7 Teenage Health and Fitness Questionnaire 142

6.2 The User Guides 142

6.3 The Trials Programme 143

6.4 Future Recommendations 147

$\begin{array}{lll}\text { CHAPTER } 7 & \text { CONCLUSION } & 151\end{array}$

$\begin{array}{ll}\text { REFERENCES } & 153\end{array}$

$\begin{array}{ll}\text { APPENDIX A DOMAIN DECLARATIONS } & 157\end{array}$

$\begin{array}{ll}\text { APPENDIX B GLOSSARY OF TERMS } & 159\end{array}$

APPENDIX C EXAMPLE QUESTIONNAIRES 163

C.1 HealthCheck Questionnaire 163

C.2 Stroke Questionnaire 168

C.3 Smoking Questionnaire 169

C.4 Readiness to Change Questionnaire 170

C.5 Audit Questionnaire 172

C.6 HealthCheck Staff Questionnaire 173

C.7 Teenage Health and Fitness Questionnaire 178

$\begin{array}{ll}\text { APPENDIX D THE USER GUIDES } & 181\end{array}$

D.1 QuestED User Guide 181

D.2 QuestEX User Guide 201

D.3 QuestED Tutorial 207 



\section{LIST OF FIGURES}

2.1 The questionnaire process.

2.2 This figure represents the information flow between the respondent and the author.

$\begin{array}{lll}2.3 & \text { Semantic differential ratings for three different questionnaire types. } & 12\end{array}$

2.4 A simple representation of an Expert System. 22

2.5 An expanded representation of an Expert System. 23

2.6 The Forward chaining inference process. 28

2.7 The Backward chaining process. 29

2.8 An example problem tree with two solution nodes. 31

2.9 The tree example showing the depth-first search path. 32

2.10 The tree example showing the breadth-first search path. 33

3.1 Stages in the questionnaire design process with a computer based tool. $\quad 37$

3.2 Example of the Block and Link concepts. 45

3.3 Example of the Section concept. 46

$\begin{array}{ll}3.4 & \text { The basic depth-first search algorithm. }\end{array}$

3.5 Example of the extended 'AND' branch. 49

3.6 A simplified extended 'AND' branch example implemented using 'OR' $\begin{array}{ll}\text { branches. } & 50\end{array}$

3.7 The standard depth-first search algorithm. 54

3.8 The dialog box for the single select list block type. 56

3.9 Dialog box of the summary display output. 56

3.10 The QuestED tree graph representation of the standard question order flow model. $\quad 57$

3.11 This example shows the operation of a condition goal. 64

3.12 This example shows the operation of the Info goal type. 65

3.13 The pseudo-code approach for the depth-first search algorithm. 66

4.1 The structure of QuestEX's modules 70 
4.2 The QuestEX open questionnaire file dialog box. 71

4.3 Top level Data Flow Diagram for QuestEX. 73

4.4 The basic implementation of the questionnaire inference engine. $\quad 76$

4.5 The method the inference engine uses to deal with goal references $\quad 77$

4.6 A flowchart description of the Back and Quit functions within the inference engine. $\quad 82$

4.7 An example of a Introduction goal group template 93

$\begin{array}{ll}\text { 4.8 The exit password dialog box is QuestEX. } & 99\end{array}$

5.1 The Tree View showing the important features. 110

5.2 The horizontal tree orientation. 112

5.3 The Questionnaire Editor Options dialog box. 112

$\begin{array}{ll}5.4 \text { Two property panel examples. } & 115\end{array}$

5.5 Examples of the DeleteGoal rules 118

5.6 The calculation and condition parsing algorithm 120

5.7 The View Template dialog box 122

5.8 The Create New Template dialog box. 122

5.9 The difference between the three types of template is shown by the $\begin{array}{ll}\text { objects with a dark border. } & 123\end{array}$

5.10 The process of creating a Text Entry template from a Single Select List template. 124

5.11 The Template Editor View 125

5.12 The Template Object floating dialog box. 125

5.13 The New Template Object dialog box. 128

5.14 The Template Align objects dialog box. $\quad 130$

5.15 The property dialog boxes for four types of template object. 131

5.16 The View Summaries dialog box. 132

5.17 The Summary Edit dialog box. 133

5.18 The Summary Entry dialog box. 134 


\section{Chapter 1}

\section{INTRODUCTION}

The QUEST system can be described as an adaptable questionnaire system. Its main purpose is to aid the author of a questionnaire in gaining relevant information from the target population of respondents. This document examines the concepts on which questionnaires and expert systems are based. The models and concepts behind the design of QUEST are then presented as is the implementation of these models. Finally, preliminary results of the project are presented along with possible future directions and improvements.

This chapter briefly outlines the history of the project since its start in 1992 and the motivation for such a project. Finally an outline of the remaining chapters is given.

In 1992 the LifeQuest project was instigated by Dr Chris Carey-Smith of the Department of Electrical and Electronic Engineering at the University of Canterbury and Dr Keith Carey-Smith of the Avon Medical Center. The aim of the project was to implement a Medical Lifestyle Screening Questionnaire from an expert system point of view. The declarative computer language Turbo Prolog was chosen as the development environment. Three 3rd Professional year students, Scott Marshall, Alan Simmons and Steve Penno, developed the LifeQuest program as part of a Knowledge Engineering class assignment. Another student, David Powley, developed the LifeQuest Editor program as a final year design project (Carey Smith et al., 1993).

The main aims for the project were:

1. To produce a questionnaire system that asks only those questions which are relevant.

2. To avoid the transfer of results from a paper questionnaire to computer for analysis.

3. To produce a summary for each set of questionnaire results.

The LifeQuest project was developed to run on an IBM-compatible computer in the DOS environment. The choice of this platform, along with the emphasis on functional 
aspects of the project, lead to the achievement of only limited success for LifeQuest. In particular:

1. As Prolog is a memory-hungry language by nature, the project ran into the $640 \mathrm{~K}$ DOS memory limit. This limited the complexity of the LifeQuest program and the size of the actual questionnaire data.

2. A large number of database files were required to store the complete questionnaire description. This was partly required to overcome possible memory limitations. Each part of the questionnaire could be read in, used and then freed from memory when finished. The number of files essentially limited the questionnaire editing process to one application only, i.e. the LifeQuest questionnaire.

3. A Basic Text mode user interface was used. This limited the appeal and useability of the program. While the program did what was functionally required, the appearance of the display was not particularly aesthetically pleasing.

Despite these limitations, overall the LifeQuest project was able to meet its aims.

In 1993 the project was continued as two final year projects by Tim Russ and the author. The initial aims for this version of the project were:

1. To develop LifeQuest as a Microsoft Windows application.

2. To generalise the questionnaire structure to enable its application to other questionnaire topics.

3. To expand on LifeQuest's original functionality and user friendliness.

The main advantages seen in developing the project for the Windows environment were the increased memory and user interface functions Windows provides. By operating in Enhanced mode, Windows has a far larger memory heap than DOS. This overcomes any problem with the size of a questionnaire. Being graphically based, Windows does much of the work of presenting the user interface. An attractive user interface can be quickly realised using Windows development tools. This was seen as especially advantageous for the questionnaire editor as it would behave in a similar manner to other Windows programs. A third program was added to the system, a Viewer that allows the questionnaire administrator to view the response files. This program provided means for displaying and printing questionnaire results.

After the completion of the project, feedback from questionnaire authors indicated a number of useability problems. These involved inadequate instructions in the questionnaire display program and insufficient authoring feedback in the editor. These problems were addressed by adding 'Context Sensitive Help Panels' in the display programs, a Windows help file, enhanced printing and a graphical questionnaire structure display in the editor. 
In 1994 the author continued the project as a research project. The aims of the research included:

1. To examine the questionnaire questioning process and develop a model of this process.

2. To improve the useability of the Questionnaire Editor and the Questionnaire Player

This project was instigated after the need for an adaptable health screening questionnaire was identified by Dr Keith Carey-Smith in 1992. At that time only one such system existed in New Zealand. This was the HealthCheck system developed by Dr John Litt, Senior Lecturer in General Practice, Flinders University Medical School, Australia. This questionnaire system had been designed to be used by patients whilst visiting their general practice. By means of questions and feedback on a variety of lifestyle issues, it provides patients with information on their current health status. In Dr Carey-Smith's view, the HealthCheck system was of limited use because of following factors:

1. The questionnaire content was fixed within the HealthCheck program.

2. The questionnaire was designed for a specific task. General practitioners would be limited to the health categories included in the package.

3. It is not possible for a general practitioner was make changes to the questionnaire context to better suit their own practice situation or to other types of study.

What was envisaged was a system with a set of generic questionnaire examples. These examples could then be modified to suit the practice's particular situation. For a more adventurous general practitioner, a new questionnaire could be designed and implemented in order to study different topics, e.g. a teenage lifestyle questionnaire and alcohol consumption questionnaire. The advantage here is that the system is no longer tied to any particular context. It can be used for any type of questionnaire and not just limited to lifestyle questionnaires. The disadvantage of this approach is that the information gathered by the questionnaire process is not as specific as the information gathered by a package such as HealthCheck.

Chapter two presents information from fields of research related to this project. The two main fields presented are Questionnaire Design and Expert Systems. Areas within these fields such as questionnaire types, quality of information, knowledge representation and inference techniques that are particularly pertinent are discussed.

Chapter three looks at the design of the questionnaire models involved in the project. The questionnaire process describes how all the involved parties interact in 
the system. A set of requirements for a computer based questionnaire are established and the next two sections describe two different methods for modeling the actual questionnaire process based on the requirements. The Question Order Flow model takes a flow chart style approach and the Required Information Flow model takes an information directed approach. The advantages and disadvantages of both approaches are outlined from an information processing view point.

Chapter four and five outline the implementation details of the main programs in the QUEST project. First the Questionnaire administration program QuestEX, is presented. The name QuestEX comes from the process of Questionnaire EXecution or administration. This followed by the implementation of Questionnaire editor program QuestED. This name stands for Questionnaire EDitor. Chapter five also includes a description of the QuestView program which is used to view the summarised replies of each questionnaire respondent. Chapter six presents a number of example questionnaires and their implementation. The preliminary experiences of a number of users of QUEST are discussed. From this a series of recommendations are made on how to improve the system in the future. Chapter seven finally draws a number of conclusions about the project. 


\section{Chapter 2}

\section{RELATED FIELDS OF RESEARCH}

This chapter describes a number of fields of research which have had an impact on this project. The Questionnaire and Survey Design section looks at the actual process of implementing a questionnaire or survey. It then describes a number of questionnaire types and then the types of questions that can be used within a questionnaire. The Expert Systems section looks at the definition of an expert system and how one may be implemented. The inference engine and knowledge acquisition processes are examined in greater depth.

\subsection{QUESTIONNAIRE AND SURVEY DESIGN}

Questionnaires and surveys often come in many different forms, however most go through the same stages or cycle of stages. These stages may be described as, (Oppenheim, 1966, Ch.1):

1. Decide the aims of the study and the hypotheses to be investigated.

2. Review questionnaire background information and identify target population.

3. Questionnaire design.

4. Pre-testing and revision.

5. Present the questionnaire to the target sample.

6. Analyse the replies and assemble the results.

7. Draw conclusions about the study aims and hypotheses.

The third and fourth stages often need to be repeated a number of times before a suitable questionnaire structure is obtained. The questionnaire process is described in figure 2.1 .

A number of roles can be identified within this process. For practical purposes a single person may perform a number of these roles. The roles are: 


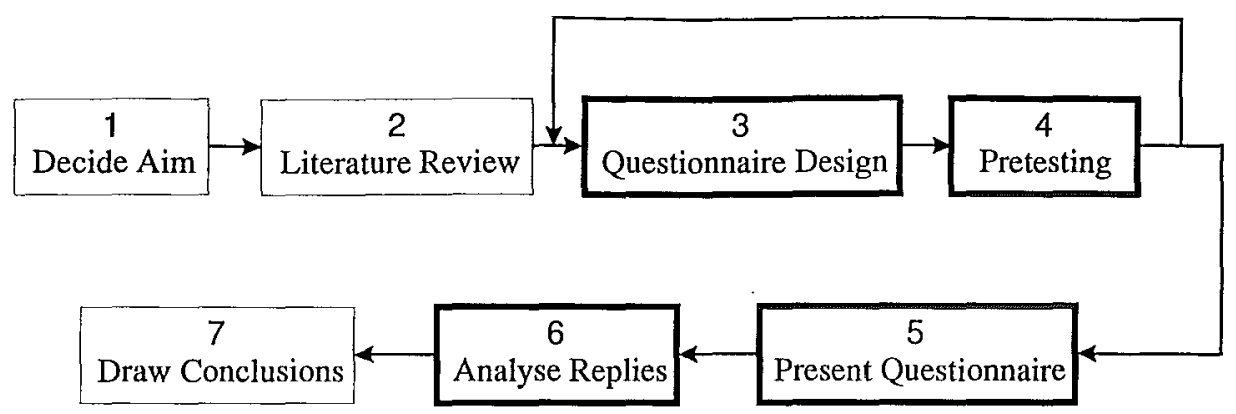

Figure 2.1 The questionnaire process.

1. Researcher. This is the instigator of the questionnaire process. This person wishes to learn information about a certain target population. The researcher will generally perform the first, second and last tasks in the questionnaire process.

2. Author. The author is responsible for designing the questionnaire's structure and questions. The second, third and fourth tasks are performed by the questionnaire author.

3. Supervisor. The role of the supervisor is to administer to the questionnaire to the questionnaire respondent. As seen in section 2.1.1 the supervisor can take an active role in presenting the question in the case of the interview questionnaire through to a completely passive role in the mail questionnaire. The supervisor plays a part in the fifth task.

4. Respondent. The respondent is a member of the sample population that the research wishes to target. The respondent also plays a part in the fifth task.

5. Analyst. The questionnaire analyst processes the respondent's raw replies into a meaningful form from which the researcher can then draw conclusions with respect to the aims of the questionnaire. The analyst performs the sixth task in the questionnaire process.

The tasks of the researcher would undoubtedly be best performed by a human expert. It is the researcher who decides the aim and direction of the questionnaire. Gathering the information relevant to the questionnaire's purpose is also best performed by a human, although computer based library catalogues can be of assistance. The final conclusion is again best drawn by the researcher who constructed the aim and hypotheses of the questionnaire.

The pre-testing task is essential when creating a balanced questionnaire. Pretesting helps reduce the bias in a questionnaire by gathering replies from a subsample of the target population. The pre-tested replies can give an indication of how the target sample may respond. Thus it gives a check on the validity and balance of the questions before actually presenting the questionnaire to the target population. If the 
questions are not satisfactory then the questionnaire design stage can be reiterated until a satisfactory result is obtained. As the pre-testing task is a combination of questionnaire design and presentation of the questionnaire to a subsample of the target population it will not be considered separately. The subsample of the population maybe chosen at random from the target population. However once this subsample has been used to pre-test the questionnaire, it may not longer be used as part of the target population when presenting the finalised questionnaire. This is because the subsample have been previously exposed to the questionnaire content during the pre-testing phase.

It is important that a questionnaire not only gathers information from the respondents, it should also provide some sort of feedback to each respondent. Feedback is important as it can maintain the interest of the respondent. An analogy would be a questionnaire administered to a respondent by an interviewer who only asked the questions. In this case the respondent would get as much information feedback from the questionnaire as if they answered the questionnaire by themselves. As soon as the questionnaire administrator provides more information than just the questionnaires, the respondent is rewarded for supplying the desired information.

The information provided by the administrator falls into two categories:

1. Comprehension Related. This type of information relates to the understanding of the questions by the respondent. To gain an accurate reply, the author must ensure that the questions are clear and unambiguous. An administrator can help here by rephrasing the question so the meaning is the same but the words are more understandable. This includes the explanation of the meaning of phrases and words.

2. Context Related. Contextual information relates directly to the questionnaire. For example, a section introduction will contain general information about a group of following questions. This falls into two further categories:

- Pre-response information. This information will be given prior to the questions being asked. It will provide the justification for asking such questions. The most fundamental example of this type of information is an actual question.

- Solution or Advice information. This is normally given once a number of questions have been answered.' The questionnaire administrator can then provide further context related information depending on the replies to the earlier questions. For example, in a sales questionnaire, once the administrator has determined the respondent is interested in a certain product, extra information can be given on that product.

It is obvious that the pre-reponse context related information must always be given. The comprehension related information is only given when the respondent fails 
to understand the context related information. An administrator can dynamically adjust the comprehension information to the respondent if there is a problem.

The concepts of information gathering from the author's point of view and information feedback from the respondent's point of view produces a two way flow of information. This information flow is shown in figure 2.2. As the questionnaire proceeds, comprehension information is given to the respondent who replies with a response. The administrator can then provide additional feedback information to the respodent.

Respondent

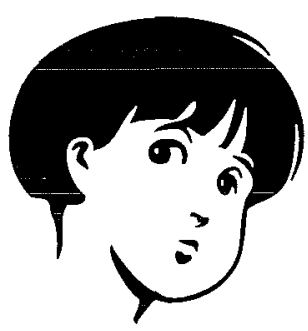

Comprehension Information

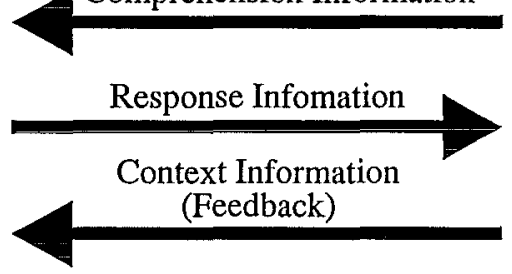

Proceeding Questionnaire
Researcher

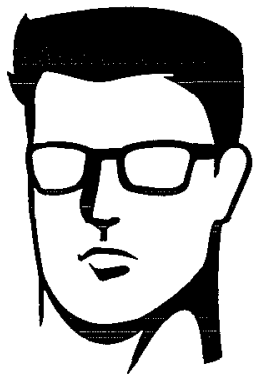

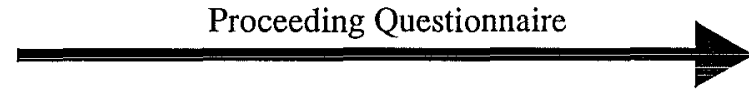

Figure 2.2 This figure represents the information flow between the respondent and the author.

\subsubsection{Questionnaire Types}

There are four main methods of data collection using a questionnaire. These are the questionnaire interview method, mail questionnaire, self-administered and group administered questionnaires. Each method has advantages and disadvantages for obtaining high quality information from the questionnaire respondent. Each method requires three components: the respondent, the supervisor and the questionnaire. Depending on the method, the supervisor players a greater or lesser role.

\section{The Interview Questionnaire}

The interview is used in many different situations for obtaining information from an interviewee. Examples of this are employment, medical and television interviews. However only data gathering interviews are described here. The questionnaire supervisor here functions as the interviewer, it is their job to present the questions to the respondents and to record the answers and replies. The interview questionnaire has a number strong advantages, however it also has a number of equally strong disadvantages. The main advantage is in the flexibility a skilled interviewer can bring to the interview. The interviewer can build and maintain rapport with the respondent during the interview and this will maintain the respondent's interest and motivation in the interview at hand. The interviewer can ensure the respondent has understood the questions and 
can perform initial classification of the replies. This is called field coding. The interviewer can probe the respondent further when certain replies are given. Above all the interviewer can interact with the respondent to achieve a high quality of data for each respondent.

The interview questionnaire is very open to the possibilities of bias even though each respondent hears the same questionnaire and the interviewing procedure is standardised. The following list describes some situations where bias may occur in a interview questionnaire:

- The interviewer may give a hint of their own views or expectations by their tone of voice or appearance. Agreeing with the respondent or showing surprise also reveal the interviewer's own attitudes.

- The manner in which the questions are presented in terms of inflection and pauses may influence the respondent.

- Leading questions and selective probing can effect balance of the original questions.

- The interviewer may misinterpret the respondent's replies or may fail to follow the questionnaire instructions.

- The interviewer may simply react differently to different respondents which consequently may unconsciously change their questionnaire delivery.

Other disadvantages are in the cost of the interviews. The interviewers must be sufficiently trained, briefed, organised and probably paid. As an incentive, the respondents may also need to be paid. The test sample of respondents maybe spread over a large area and for a large sample group this may take a long period of time.

\section{The Mail Questionnaire}

The supervisor of a mail questionnaire plays far less of a role than the interviewer in the questionnaire interview. To a large extent the respondent must supervise themselves. This means there will be no interviewer bias, however the questionnaire must be much simpler because there is no interviewer to provide further explanations. Because of this simplicity, the later processing and analysis is usually easier to do than for an interview type questionnaire. The main advantage of the mail questionnaire is its low cost. It does not require a trained interviewer to visit every member of the target population. Most of the cost of a mail questionnaire is mainly in the planning, printing and postage of the questionnaire. So for a lower cost, a mail questionnaire can reach a far greater target population than the interview questionnaire. This can result in greater sampling accuracy of the population. 
There are a number of disadvantages because there is no supervisor or interviewer present. It lacks the personal touch an interviewer, so it can be difficult to build a rapport with the respondent. There is nothing stopping the respondent from reading the mail questionnaire before they attempt to answer it. This may lead to a biased result if the respondent develops a series of replies before actually answering the questionnaire as it was intended. Respondent may come back to questions left earlier because they were too difficult or required further thought. This may bias the replies especially if later questions follow a particular line of questioning. The sampling of the population may be distorted if the intended questionnaire respondent passes the questionnaire onto someone else. While the other person maybe clearly more appropriate, this may effect the sampling especially if the sample population was chosen specially.

A major problem mail questionnaire suffer is response rate. Without a supervisor to ensure completion of the questionnaire a large proportion of the sample population will fail to return a reply. This will further introduce bias into the final analysis because the questionnaires which are returned no longer represent the target population as it was first chosen. This is because non-response is not a random process (Oppenheim, 1966, Ch. 2).

\section{The Self-Administered Questionnaire}

A self administered questionnaire combines many of the advantages of both the mail questionnaire and the interview questionnaire. The role of the supervisor in this case is basically the supervising of the respondent while answering the questionnaire. This gives the benefit of personal contact and allows the explanation of questions. Note that this does not include the interpretation of questions. This contact ensures a high response rate, accurate sampling and a minimum of interviewer bias. The supervisor does not need to be a trained interviewer, however the supervisor must not introduce bias unwittingly. The questionnaire would have a similar complexity to the mail questionnaires, as on the whole the respondent has to attempt it by themselves. However the possibility still exists that the respondent may misinterpret questions and make mistakes. The respondent can still read the questionnaire through before attempting to answer the questions which may bias the replies.

\section{The Group-Administered Questionnaire}

Group administered questionnaires combine many of the advantages of the self administered questionnaires and the questionnaire interview method. It is also useful as a large group of people can take the questionnaire at one time. More than one supervisor maybe required to help with question explanations and to check completed questionnaires. One possible advantage is that extra background information can be presented to the group in the form of a presentation. The questions may be read out to the 
respondents one at a time. This will ensure that every one in the group has the same amount of time for each question and that they do them in the same order. The main disadvantage is the risk of bias through interacting with other individuals in the sample group. This may occur through asking questions, talking and copying.

\section{Computer Questionnaires}

Computer questionnaires are normally administered by a computer program. This makes them most like interview questionnaires as the respondent answers a questionnaire completely administered by the computer 'interviewer'. The computer questionnaire has the interview questionnaire disadvantage of high cost, however this is normally the one off capital cost of purchasing the computer equipment. On the other hand it does not have the interviewer problems of turnover, error and bias.

Importantly the computer questionnaire can provide a consistent interface to all the respondents, thus reducing interviewer error and respondent interpretation error (Slack et al., 1966) . Respondent interpretation error and bias are still a problem as the flexibility provided by a human interviewer is lacking. The computer questionnaire can still probe for more information and give feedback to the respondent. For example it can be used to educate and inform the respondent as well as gather information. However this probing and feedback is programmed and not spontaneous as a human expert could provide.

The computer questionnaire lacks the same rapport the human expert can have with the respondent. However it benefits from the 'game effect' and anonymity. Although many people do not react positively to computers, many find them interesting, challenging and fun to use. Computer games are popular partly due to the use of colourful graphic displays and interactive feedback of information to given prompts and actions from the user. These sort of effects can provide a motivation or rapport factor not present in the other questionnaire types. Interactive feedback in a computer questionnaire is useful in a education role (Allen and Skinner, 1987). These types of advantages are shown in figure 2.3 which is a rating graph of a number of sematic differential items (cold-friendly, short-long etc. etc.) from a alcohol, drug and tobacco use study which used a computer questionnaire as the data gathering tool (Skinner and Allen, 1983).

One area in which computer questionnaires have been successfully used is health screening and risk assessment. Computer screening programs have been used to identify various at risk groups within a medical practice (Moughan, 1988). Many respondents react more positively towards a computer questionnaire when faced with difficult or possibility embarrassing situations or topics. This agrees with tests using MediaMediated interview (computer and/or video interfaces) techniques. This suggests that 


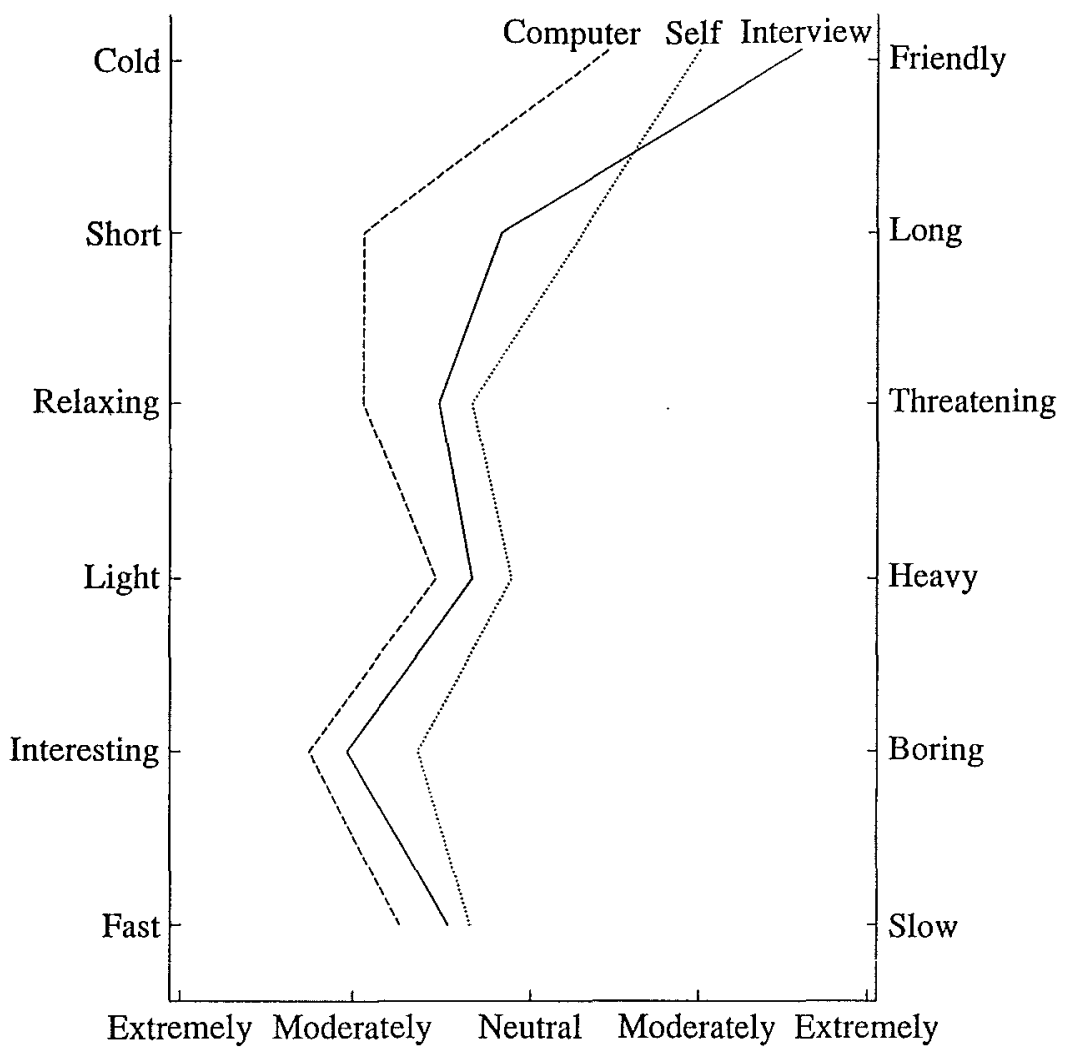

Figure 2.3 Semantic differential ratings for three different questionnaire types.

such interviews induced talkativeness in the respondent when compared with face-toface interviews (Yoshida et al., 1993).

A further advantage of computer questionnaires comes during the collection of the respondent's replies. With a paper questionnaire, the replies are normally entered in a database before being further processed and analysed. This requires skilled data entry workers who can rapidly and quickly enter the respondent data. A computer questionnaire on the other hand can save the respondent's replies immediately after then questionnaire has been completed and without data entry errors. These replies are then available for corrections, analysis and report building.

In summary some of the main advantages and disadvantages of these five types of questionnaire are given in table 2.1. Each of these questionnaire types is suited to different situations:

- Interview. Best with experienced interviewers and when detailed and thorough information is required from the respondents.

- Mail. Good when low cost is a factor, a large sample population is reachable by mail, respondent error is acceptable but response rates are often poor.

- Self. Good when the respondents can be gathered together and have sufficient literacy and comprehension skills to complete the questionnaire. 
- Group. Good when a large group of respondents are gathered in one place and when a controlled delivery of the questionnaire is possible.

- Computer. Best questionnaire type when respondents answer the questionnaire individually. Ensures a controlled and consistent delivery of the questionnaire.

Table 2.1 Advantages and disadvantages of five types of questionnaire.

\begin{tabular}{|c|c|c|}
\hline Type & Advantages & Disadvantages \\
\hline Interview & $\begin{array}{l}\text { - Flexibility } \\
\text { - Explanation } \\
\text { - Probe } \\
\text { - Rapport } \\
\text { - Motivation }\end{array}$ & $\begin{array}{l}\text { - Interviewer bias } \\
\text { - Interviewer error } \\
\text { - Interviewer turnover } \\
\text { - High costs }\end{array}$ \\
\hline Mail & $\begin{array}{l}\text { - Low Cost } \\
\text { - Large Sample } \\
\text { - Accurate Sampling } \\
\text { - No Interviewer Bias }\end{array}$ & $\begin{array}{l}\text { - Poor response rate } \\
\text { - Respondent error } \\
\text { - Respondent bias } \\
\text { - Lacking personal touch } \\
\text { - Respondent }\end{array}$ \\
\hline Self & $\begin{array}{l}\text { - High response rate } \\
\text { - High accuracy } \\
\text { - Minimal interview bias } \\
\text { - Don't need skilled interviewer } \\
\text { - Supervisor provides explanations }\end{array}$ & $\begin{array}{l}\text { - Unskilled supervisor bias } \\
\text { - Respondent bias } \\
\text { - Respondent interpretation error }\end{array}$ \\
\hline Group & $\begin{array}{l}\text { - Sample of respondents can be } \\
\text { controlled together } \\
\text { - Similar benefits as Self }\end{array}$ & $\begin{array}{l}\text { - Tainted replies through talking, } \\
\text { copying and asking questions }\end{array}$ \\
\hline Computer & $\begin{array}{l}\text { - Anonymity for respondent } \\
\text { - Complex skipping patterns } \\
\text { - 'Game' effect } \\
\text { - Programmed probe ability } \\
\text { - Programmed explanation } \\
\text { - No interviewer bias } \\
\text { - Convenient analysis }\end{array}$ & $\begin{array}{l}\text { - Fear of Computers } \\
\text { - Author bias } \\
\text { - Setup costs } \\
\text { - Computer illiteracy } \\
\text { - Lack of rapport, impersonal }\end{array}$ \\
\hline
\end{tabular}

\subsubsection{Quality of Information}

Loss of information is always going to occur in a questionnaire. This loss of information will lower the quality of the final information produced during the questionnaire analysis phase. The best that can be done is to minimise the loss. This can be done by understanding how the loss occurs and accounting for it. There are three important factors which affect the quality of the information gathered by a questionnaire:

1. Respondent Bias and Error. Respondent bias arises through a number of factors. Background factors influence how the respondent will approach the interview. Each respondent will have fixed attitudes, personality characteristics, motives 
and goals. These may come from various group memberships and loyalties such as age, sex, race, religion, income and education. Psychological factors such as the respondent's sensitivity to the interviewer's attitudes, and in particular the interviewer's attitude to them is likely to be a great source of potential bias. The respondent's behaviour which may cause bias is almost entirely due to their reaction to the interviewer's behaviour.

2. Interviewer Bias and Error. Interviewer bias is a problem which consists of two parts: the first part relates to the question, and the second to the question delivery. In the design of the questions bias can be introduced simply through the choice of words in the question. For example a question on the state of the economy can be biased towards well educated people if it used complex language and jargon. In a closed question, the choices of the predetermined replies influence how respondents answers the question. By not including a particular relevant reply, the respondent may be forced into choosing a reply they normally would not (Labaw, 1980, Ch. 13). It has even been suggested that even asking a question may motivate a respondent to form new opinions or change existing opinions (Bridge and $\mathrm{Al}, 1977)$. The Interviewer can also bias the respondent's reply through the question delivery. Interviewer bias can arise from errors in asking the question, incorrectly probing the respondent for further information and errors in recording the respondents replies. Motivation of the respondent by the interviewer can lead to bias as the interviewer may under-motivate the respondent to provide worthwhile answers. Alternatively the interview may over-motivate or inappropriately motivate the respondent to give replies other than they normally would (Kahn and Cannell, 1957, Ch. 7). The interviewer may bias the results simply by not being able to accurately record all the details of the respondent's answer on paper. For example, once recorded on paper, the respondent's expressions and emphasis are lost, which may lead to a misrepresentation of what the respondent actually meant.

3. Invalid and Dirty Data. Mistakes in recording the respondent's replies lead to invalid and dirty data. These mistakes may occur initially as the interviewer records the responses or at a later stage when the responses are entered into a database. If this data is not corrected before the analysis is carried out, then erroneous conclusions will result. Some of this data is obviously incorrect as it lies outside of an acceptable range. This is called dirty data and it often can only be corrected by re-asking the question to the respondent. Data that is not obviously incorrect may never be detected and thus will affect the final results.

The quality of the respondent's replies can also be effected by their attitude to computers. While some people find computers interesting and fun, other people do not 
react well to computers. This can effect the quality of the final questionnaire analysis and will result in a bias towards these people more computer literate. A number of factors may contribute to this problem:

1. Lack of Motivation. This may arise from a number of sources, for example:

(a) Boredom. A person who is not interested in the task at hand will not put the effort to their replies, thus resulting in a loss of quality. A boring computer questionnaire will be as bad as a boring paper questionnaire in this respect. However a multimedia capable computer questionnaire should able to interest most people when the questionnaire is well designed.

(b) Apathy. Again a problem for all forms of questionnaires and a more difficult problem to solve than boredom as it requires a change in the respondent's attitude.

2. Fear of Computers. Many people have an unwillingness towards things they know little about. This can cause a reluctance to use an unfamiliar appliance. Computers appear to raise this reluctance more than most other products of new technology. Although computers have been in use in various forms for the last fifty years, only now are they becoming widely used and accepted. Initially most people saw computers as machines only used by a small sector of society. This was compounded by the amount of knowledge and training that was required to operate the early computers. In the last ten years, the miniaturisation of computers has lead to their proliferation into the 'home computer' market. With people starting to use and see computers in the home environment they are becoming less reluctant to use them. However a large number in the population who haven't grown up with computers still fear this 'new' technology.

3. Confidentiality. It seems a fair assumption that if you can't always trust a person with your confidential details, you can't trust a computer with the same information. As the issue of privacy has arisen in recent times it is important to safeguard any sensitive information from the possibility of illegal access. Unfortunately it isn't possible to give the Hippocratic oath to a computer. In the end it is the computer operator's responsibility for ensuring confidentiality and using any available measures to achieve this.

\subsubsection{Question Types}

There are two categories of question types with respect to the question wording. These are open questions and closed questions. In general, all questions are either open or closed. An open question requires the respondent to give a free response which is not governed by any sort of choice. The main advantage of the open question is 
the freedom the respondent has while answering the question. Once the question has been fully understood, the respondent can answer in their own words to express their feelings in relation to the question. In has been shown that open questions have an advantage over closed questions in certain subjects. For example it has been shown that estimates for drinking and sexual activity were more accurately reported when using open questions (Bradburn et al., 1979, Ch. 2). Closed questions included response categories such as "never, once a year, ever few months, monthly, once a week, several times a week and daily". It appeared that people were less likely to admit to the high frequency categories in the presence of the low frequency categories. The open form is also preferred when not enough information is known to make appropriate response categories (Converse and Presser, 1986, Ch. 2). In this situation poorly chosen response categories for a closed question will produce biased results from the respondent.

There are a number of disadvantages for the open question form. Open questions are easy to design and ask, but due to their imprecise nature, they can be very difficult to use in the final analysis. The open question responses are generally classified or 'coded'. This can result in a loss of information and in the richness of the respondent's replies. This step can be quite involved and requires traincd staff in an attempt to minimise the amount of information lost. Another disadvantage is the risk that the respondent will answer what happens to be uppermost in their mind. So if the respondent is influenced by the interviewer or the questions then the replies can be biased. The respondent may not provide full answers as they may be put off by having to write long written responses. The open question appears more appropriate when the objective is to learn the respondent's attitude, their level of information on which the attitude or opinion is formed, the frame of reference within which the question, and the intensity of their feeling (Kahn and Cannell, 1957, Ch. 6).

Closed questions give the respondent a choice from a list of alternative replies. The respondent may select one or any number of the replies. Typically a closed question may be as simple as a Yes/No question or as complex as a graduated scale for measuring an intensity response. The respondent is expected to choose the alternative reply which comes closest to or best represents their feelings, attitudes or knowledge of a situation.

The main advantage of closed questions is in their simplicity. A closed question will generally only be interested in a single concept while a open question maybe interested in a number of concepts. This has the benefits that the question is quicker and easier to answer for the respondent. More questions can be answered in a shorter period of time and the replies can be analysed more readily and at a cheaper cost. Closed questions being more specific than open questions are more likely to communicate the same frame of reference, or put the all respondents in the same frame of mind. This leads to more consistent replies across the sample population. Compared to an open question, the closed question maybe more difficult to formulate as a balanced set of replies must be found the question. Finding these replies maybe require several iterations of pre- 
testing or pilot testing on a group of test respondents. The different replies from the test respondent can be used to define the different replies. However this approach will reduce any ambiguous respondent replies that may occur with open questions. For example the question "People look for different things in a job. What would you most prefer in a job?". If the question was open, and a reply was "the pay", then this is ambiguous as it could mean either "high pay" or "steady pay". A closed question that includes these categories amongst others, would solve this problem as the more specific replies would give the respondent a better idea of the nature of the question.

However the closed question also has disadvantages (Converse and Presser, 1986, Ch. 2). The respondent is forced to answer a question with a fixed number of choices. The predefined replies may irritate the respondent if they feel the replies fail to do justice to their own ideas (Oppenheim, 1966, Ch. 2). These choices may introduce bias if the predefined replies are not carefully chosen. The replies may lead to respondent into a certain frame of mind which may then influence the way later questions are answered.

The closed question is appropriate when our objective is limited to the classification of the respondent with respect to some simple attitude or perception. The more complex and ambitious the objectives the harder and less satisfactory it is to formulate simple and effective closed questions when a single open question may suffice feeling (Kahn and Cannell, 1957, Ch. 6).

It is also often advantageous to ask open and closed questions together. When used in this manner the closed questions provide a statistical count and the open questions provide the meaning of the statistical count (Labaw, 1980, Ch. 13). Using an open follow up question to a closed question can reveal flaws in the in choice of replies in the closed question. The open question can indicate if the respondent did not completely comprehend the either closed question or the predefined replies. On the other hand by asking an open question first will give a response in the respondent's own language and containing their own ideas. Although this is useful, it is difficult to compare with answers from other respondents. Also there is no certainty that the answer contains all the factors important to the respondent. So a similar closed question can then be asked which may contain predefined answers that did not earlier occur to the respondent. This approach ensures that the results of several respondents can be readily be compared and that all respondents have considered the 'universe of content' before giving their replies (Oppenheim, 1966, Ch. 2).

\section{Closed Question Types}

There are a number of types of closed questions. Their main feature is that they do not ask the respondent to write anything, although they may require the respondent to read and think about the question. The question usually contains a list of checks or 
choices, from which it is possible to calculate a score. These are more suitable for mail, group and computer questionnaire types. for A number of different question types are listed here (Oppenheim, 1966, Ch. 4) :

- Checklists. These contain terms which the respondent can easily understand and which more briefly and compactly expresses their views than their answer to an equivalent open-ended question. They are best used when testing specific hypothesises as they can be difficult to interpret. For example,

Please give your opinion on these attributes of a prospective husband:

\begin{tabular}{l|l|l|l|l|} 
& V. Important & Important & Indifferent & Undesirable \\
\hline 1. Good looks & & & & \\
\hline 2. Kindness & & & & \\
\hline 3. Money & & & & \\
\hline 4. Dependability & & & & \\
\hline 5. Sense of humor & & & & \\
\hline 6. Ambition & & & & \\
\hline 7. Moral character & & & & \\
\hline 8. Domesticity & & & & \\
\hline 9. Intelligence & & & & \\
\hline 10. Good health & & & & \\
\hline
\end{tabular}

- Ratings. Similar to checklists but they are designed to give a numerical value to some kind of judgement. Ratings can be used as: an objective assessment of a particular item of interest, an indication of the respondent's attitudes or understanding, or as a self-rating of the respondent themselves. For example,

Out of 5 , how would you rate the last film you saw:

\begin{tabular}{cc}
1. & Outstanding \\
2. & Above Average \\
3. & Average \\
4. & Below Average \\
5. & Disappointing \\
\hline
\end{tabular}

- Rankings. This means to arrange a list in order with respect to some common aspect. Ranking involves a comparison between the items in the list to decide the order of the ranked list. Rankings are more useful when telling us something about the respondent rather than the list being ranked. This is because ranking tells nothing of the difference between ranks which is as important as the rank sequence. Also the actual comparison maybe difficult especially when the list has a large number of items. For example tennis players are 'ranked' according to their performances over a year of tournaments. 
- Inventories. An inventory of a list of items which the respondent is asked to check in a particular way. Often the respondent is asked to indicate which items in the list apply to them. They are relatively crude, as if can be difficult to make a balanced list of items which apply well to a large category of the respondent population. However it can be useful way of gathering broad information on a wide series of topics quickly. For example,

Does any member of you family have any of the following problems either now, or in the past?

\begin{tabular}{ll}
\hline 1. & Heart attack or angina \\
2. & High blood pressure \\
3. & Bowel cancer \\
4. & Diabetes \\
5. & Asthma \\
6. & Melanoma \\
7. & Alcohol problems \\
\hline
\end{tabular}

- Grids. A grid can be though of as a two dimensional inventory. It is a simple method of quickly gather information without having to ask a lot of questions. For examples a grid might have a number of illnesses and complaints on one axis and common remedies on the other axis. With each respondent checking those remedies they would use for each illness a picture can be built up of the preferred treatments and situation they are applied under.

\subsection{EXPERT SYSTEMS}

The field of Expert Systems is a part of the larger field of Artificial Intelligence (ÁA). "In the simplest sense, AI is the study of developing computer programs that exhibit human-like intelligence" (Durkin, 1994, Ch. 1). The initial work done in the field of AI in the 1950's was based on computational logic. From this attempts were made to develop general purpose systems which could solve a variety of problems. However this general purpose approach failed to deliver the promised results because of two main reasons (Durkin, 1994, Ch. 1):

1. The limited performance of computers in the 1960's.

2. The general-purpose problem-solving strategies of the time were too weak for complex problems.

After a low period in the 1970's, research began to pick up in the field of AI when researchers began to realise that intelligent behaviour is dependent on the knowledge one has to reason with rather than the methods of reasoning. This led to the concept of knowledge-based or expert systems (Durkin, 1994, Ch. 1). 
Two lessons were learnt from research into the field of AI:

1. General-purpose reasoning techniques were too limited to solve real problems.

2. Systems which were focused on simple issues performed better than generalpurpose systems.

These led to the definition of an Expert System: 'Solves problems using a computer model of expert human reasoning, reaching the same conclusions that a human expert would reach if faced with a comparable problem' (Weiss and Kulikowski, 1984, Ch. 1).

\subsubsection{Motivations for making an Expert System}

The definitions indicate that an expert system somehow incorporates the knowledge of a human expert. The expert system is also able to apply this knowledge to a particular situation to produce a satisfactory solution. Given that a human expert can also produce these results, why use expert systems? There are two general reasons why an expert system might be built (Durkin, 1994, Ch. 1):

1. Replacement of a Human Expert. While this is not a particularly good option for the human expert, there are a number of advantageous reasons for this:

- Make available expertise after hours or in other locations. An expert system can operate all hours of the day. It can be cheaply duplicated and distributed to areas where there is no human expert.

- Automate a routine task requiring an expert. An expert system will produce the same quality of results no matter how many times the task is performed.

- Record expertisc. If a human expert has retired, taken a new job or has died, then their expertise is no longer available. Recording the expert's knowledge allows their expertise to be used further or passed on.

- Reduce costs. Human experts are expensive to train and to retain. An expert system's greatest expense is in development. The subsequent maintenance costs of the expert system will be much less than the salary of the human expert.

- Provide expertise in hostile environments. An example of this is the DENDRAL expert system. This was developed for the NASA unmanned missions to Mars. DENDRAL was able to provide expert chemical analysis of the Martian soil.

2. Assisting a Human Expert. Assisting a human expert is a more common application for an expert system. This situation is desirable when the human expert is capable of performing the task, but gets support from the expert system. The reasons for doing this are: 
- Improving productivity. A human expert would be aided by letting an expert system initially perform the task at hand. The expert system would be able to provide an initial attempt at a solution which the human expert can review. The human expert is fully able to perform the task, but an expert system would provide additional support.

- Provide effective management of complex problems. A human expert may have difficulties in keeping all the variables from a complex problem in mind, for example, in project management. An expert system has no limit other than program memory on the number of variables it can keep track of.

- Consistent recall of information and knowledge. A human expert would tend to forget information and knowledge used infrequently. A expert system remembers all information equally, so would have no difficulty applying knowledge from extreme or exceptionally cases.

While these reasons aid the productivity of a certain task there are more motives for building an expert system. These go beyond the productivity considerations (Weiss and Kulikowski, 1984, Ch. 1):

1. Disseminating rare and costly expertise. With the increasing use of low cost microcomputers, the development of expert systems enables expertise to be shared amongst a larger population. Human experts are often in short supply and when available have little time available. The human expert's advantages lie in the flexibility of human response and the ability of sensory pattern recognition. While an expert system can not even attempt to match a human in these areas, an expert system can record the systematic approach of a human expert. A situation where a human non-expert provides the perceptual input and an expert system processes a solution is better than not having any form of expert assistance.

2. Formalising expert knowledge. Human experts often work in areas where scientific knowledge lags behind practical knowledge. These experts attempt to solve problems without a detailed scientific understanding of the problem. The expert may suggest experiments that will support or reject ideas about the problem. These ideas maybe developed with further experiments on other instances of the problem. By formalising the expert's knowledge, an expert system can reproduce the human expert's reasoning producing further alternatives for experimentation. The results of these experiments can then further add to the practical knowledge of the problem.

3. Integrating diverse sources of knowledge. Due to different backgrounds, different human experts often have differing opinions with different approaches when solving problems. Each expert's approach may be just as valid as the next expert. By incorporating different expert's opinions on a problem into an expert system the 
quality of the expert system's knowledge base is increased. This is because the expert system can then provide more alternative solutions. While some solutions may not as appropriate given a certain situation, the knowledge the solution is based on is not irrelevant. This knowledge however may be more appropriate in another situation. So by integrating diverse sources of knowledge the expert system can better represent the state of human knowledge.

\subsubsection{Expert System Structure}

It is apparent that an expert system contains at least:

1. Knowledge Database.

2. Reasoning or Inference mechanism.

3. User.

The relationship between these components is shown in Figure 2.4

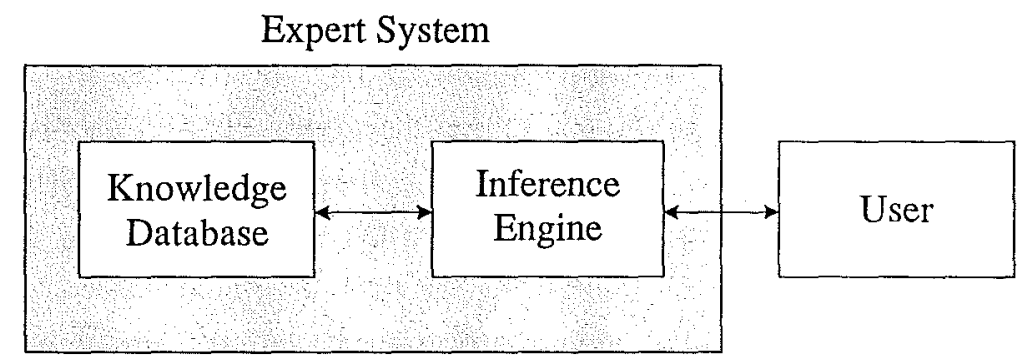

Figure 2.4 A simple representation of an Expert System.

The Knowledge database holds the two types of information. These can be termed Knowledge Base and the Working Memory. The Knowledge base can be defined as "Part of an expert system that contains the domain knowledge" (Durkin, 1994, Ch. 2). This is analogous to the long term memory of a human expert. The Working Memory can be defined as "Part of an expert system that contains problem facts that are discovered during the session" (Durkin, 1994, Ch. 2). This is analogous to the human expert's short term memory. The facts in the working memory typically are either information entered by the user of the system or information inferred by the expert system's inference engine. The inference mechanism is usually called an Inference Engine. It can be defined as "The processor in an expert system that matches facts contained in the working memory with the domain knowledge contained in the knowledge base, to draw conclusions about the problem" (Durkin, 1994, Ch. 2). The user of an expert system provides the initial set of conditions for the solution by inserting a set of facts into the working memory. The inference engine uses these facts and the knowledge in the knowledge base to produce a new set of facts. This inference process 
may also require extra information from the user, which once obtained can result in further facts. This process of obtaining facts and inferring new facts continues until a goal state is reached. This goal state is the inferred solution to the problem.

This simple representation of an expert system can be further expanded as shown in Figure 2.5.

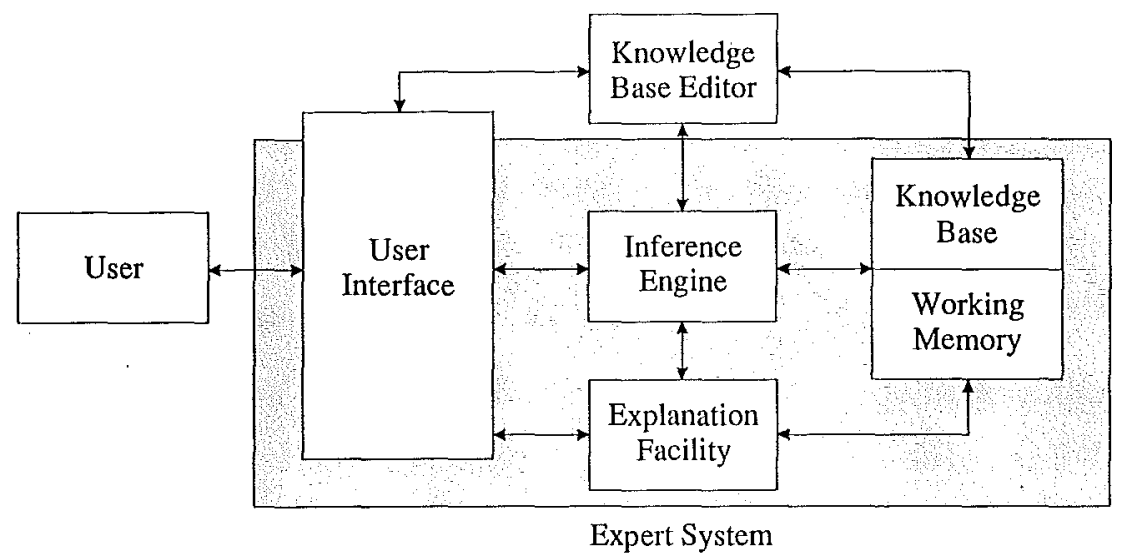

Figure 2.5 An expanded representation of an Expert System.

This introduces three more components, these are:

1. User interface.

2. Explanation facility.

3. Knowledge Base Editor.

The User Interface provides the mechanism which allows the inference engine to obtain facts from the user. This provides either a natural language, graphical, menu driven or question-and-answer interface to the user. Feedback of information also occurs through the user interface. The Explanation Facility generally can indicate to the user why and how a particular question is being asked. A why explanation can make the user more comfortable with the line of questioning and can provide insight into what issues the expert believes are important. The why explanation attempts to indicate the conclusion the system is working towards. A how explanation provides justification for a particular conclusion. It displays the facts and rules used to arrive at the conclusion. The Knowledge Base Editor allows the human expert to modify the knowledge base. This allows the addition of new knowledge, helps to maintain correct knowledge syntax and performs consistency checks on the new knowledge information.

\subsubsection{Knowledge Representation}

The Knowledge base contains the knowledge or information relevant to the task of the expert system. In this sense knowledge can be defined as an 'Understanding of a 
subject area' (Durkin, 1994, Ch. 3). In order to use this knowledge in a computer program, a way of structuring the knowledge must be found. This structure must allow the computer program to apply this knowledge in a similar way to how a human expert would. This structuring of the knowledge is known as knowledge representation. It has been defined as 'The method used to encode knowledge in an expert system's knowledge base' (Durkin, 1994, Ch. 3).

Just as there are a large number of potential applications for expert systems, there are also a large number of ways to structuring the knowledge for each application. Although the type of knowledge for each application may be different, it has been found that many of the knowledge types are similar and can be categorised. These categories are (Durkin, 1994, Ch. 3):

1. Procedural knowledge. This describes how to solve a problem. Rules, strategies, agendas and procedures are used to provide direction on how to do something.

2. Declarative knowledge. This describes what is known about a problem. A list of true or false statements are used to describe a concept or object.

3. Meta-knowledge. This is knowledge about knowledge. This describes how to represent knowledge in an attempt to efficiently use that knowledge. Properly described and structured knowledge allows the inference or reasoning process to proceed more efficiently.

4. Heuristic knowledge. This describes a rule-of-thumb that is used to guide the reasoning process. Generally a human expert will formulate heuristic knowledge from past experience of solving similar problems.

5. Structural knowledge. This describes how knowledge siructure which often represent a human expert's model of the problem. The human expert's model of concepts, sub-concepts and objects is typical of this type of knowledge.

Given these types of knowledge different methods of representing the knowledge have evolved. Mylopoulos and Levesque (1984) have classified four types of representation schemes (Brodie et al., 1984):

1. Logical Representation schemes. This class of representations uses expressions in formal logic to represent a knowledge base. Inference rules and proof procedures apply this knowledge to problem instances. Examples of this are first order predicate calculus, propositional logic and reasoning with logic.

2. Procedural representation schemes. Procedural schemes represent knowledge as a set of instructions for solving a problem. This contrasts with the declarative representations provided by logic and semantic networks. Rule based systems and production systems are examples of procedural representation schemes. 
3. Network representation schemes. Network representations capture knowledge as a graph in which the nodes represent objects or concepts in the problem domain and the arcs represent relations or associations between them. Examples of this are sematic networks, conceptual dependencies, conceptual graphs and inheritance networks.

4. Structured representation schemes. Structured representation languages extend networks by allowing each node to be a complex data structure consisting of named slots with attached values. Examples of this are scripts, frames and objects.

\subsubsection{Inference Techniques}

An inference mechanism or engine in an Expert system is used to combine the knowledge in the knowledge base with the facts from the working memory. The result of this combining in new knowledge or facts which can be placed back into the working memory. This process starts normally with a well defined goal in mind which the process must be able to recognise. The process then repeats until this goal has been achieved. This inference process must be controlled in order to produce an efficient solution (Durkin, 1994; Parsaye and Chignell, 1988). Various control strategies have been established to identify the goals of the system and to guide the system's reasoning towards the goals.

The term 'inference' is the name of the technique used in expert systems to model the reasoning process human experts use to solve a problem. A number of forms of human reasoning are presented in the next section. The following sections then describe a number of techniques used in the inference engine of an expert system.

\section{Reasoning}

Human reasoning is 'the process of working with knowledge, facts, and problem solving strategies to draw conclusions'. Understanding how humans reason provides insight into how to guide knowledge processing in an expert system. There are five main forms of human reasoning (Durkin, 1994, Ch. 4):

- Deductive reasoning. This the deduction of new information from logically related known information. The basic form of deductive inference is the modus ponens rule. For example,

If $A$ is true and if $A$ implies $B$ is true, then $B$ is true.

- Inductive reasoning. This is used to arrive at a general conclusion from a limited set of facts through the process of generalisation. A generalisation is formed 
which we believe applies to all cases of a certain type, on the basis of a smaller number of cases. For example,

For a set of objects, $X=\{a, b, c, d, \ldots\}$, if property $P$ is true for $a, P$ is true for $b$, and if $P$ is true for $c, \ldots$ then $P$ is true for all $X$.

- Abductive reasoning. Abduction is a form of deduction that forms conclusions which might follow from the available information, but it might also be wrong. For example, If $B$ is true and if $A$ implies $B$ is true, then $A$ is true?

- Analogical reasoning. In this form of reasoning, humans draw from the differences and similarities in their knowledge and experiences to guide their reasoning about some new knowledge. For example if we know information about a certain object $A$, if we are then told that object $B$ is like object $A$ then we can draw analogies between $A$ and $B$ to form new information about object $B$.

- Common-sense reasoning. This form of reasoning replies more on good judgments than on exact logic. The good judgements are also referred to as heuristics or rules of thumb. These rules of thumb are usually formed from a human experiences. The heuristic provides a reasonable indication of where a solution may be found. However this does not guarantee that a solution will be found, only that the direction indicated is a reasonable place to start looking for a solution.

\section{Logical Inference}

Logical inference forms a basis of two most common inference techniques, backward chaining and forward chaining. These two techniques are presented in the following two sections. Logical inference is the oldest form of knowledge representation in computers. Propositional logic and predicate calculus are two forms of logic which have been well used in the artificial intelligence field. Both techniques use symbols to represent knowledge and operators applied to the symbols to produce logical reasoning (Durkin, 1994, Ch. 4).

- Modus Ponens. The basis of modus ponens is the definition, 'Rule of logic that asserts that if we know $A$ is true and that $A$ imples $B$ is true, then we assume that $B$ is true.' Working with set of implications or rules and initial data, modus ponens forms a series of logical assertions. Thus the inference process is driven by the asserted information. This is the basis of data-driven or forward chaining expert systems. It is suited to applications where it is important to learn as much information as possible from available information.

- Resolution. Resolution is defined as 'Inference strategy used in logical systems to determine the truth of an assertion' (Durkin, 1994, Ch. 4). It was first 
introduced in 1965 by Robinson. It is based on a technique called proof by refutation which attempts to prove a proposition $\mathrm{P}$ is true by proving that the negation of $\mathrm{P}, \neg \mathrm{P}$, cannot be true. Resolution can be performed using either propositional logic or predicate calculus, although the predicate calculus form is more complex. Resolution focuses on the goal it is attempting to establish, and only considers information relevant to the proving the goal.

- Non-resolution. The process of resolution makes no distinction between goals, premises or rules. This can be confusing when attempting to prove a goal, as the goal can be lost within the resolution as easily as the premises and rules. The nonresolution or natural-deduction technique on the other hand attempts to prove a statement in a goal-directed fashion. Natural-deduction uses a back-chain rule of inference, for example,

Rule:

Lives_In(John,New Zealand) $\wedge$ Lives_In(X.New Zealand $) \rightarrow$ Likes(X,All Blacks)

Goal: $\rightarrow$ Likes(John,All Blacks)

To prove the goal, the rules are scanned for a conclusion which matches Likes( John, All Blacks). If found the conclusion becomes a new sub goal of the process. If Lives_In(X, New Zealand) implies Likes(John, All Blacks) then a new sub goal to prove is Lives_In(John, New Zealand). Natural resolution shares some of the same features as goal-driven or backward-chaining expert systems.

\section{Forward chaining}

Simply put, forward chaining attempts to find a solution to a problem by examining available facts. An obvious example is a diagnosis. Here an expert will attempt to find a set of initial general symptoms or facts. This information is then used by the expert to infer a initial conclusion or hypothesis which can be explored further. In this case the data drives the expert's reasoning process to a conclusion.

Forward chaining has been defined by Durkin as an 'Inference strategy that begins with a set of known facts, derives new facts using rules whose premise match the known facts, and continues this process until a goal state is reached or until no further rule has premises that match the known or derived facts.' This definition is shown in the flow-chart in Figure 2.6.

In this flow-chart the inference process starts with the adding of the known information into the working memory. The first rule in the knowledge base is matched against the facts in the working memory. If at least one matching rule is found then that rule is 'fired' by adding its conclusion into the working memory. If there is more than one rule match, then the conflict resolution stage determines which rule is fired. 


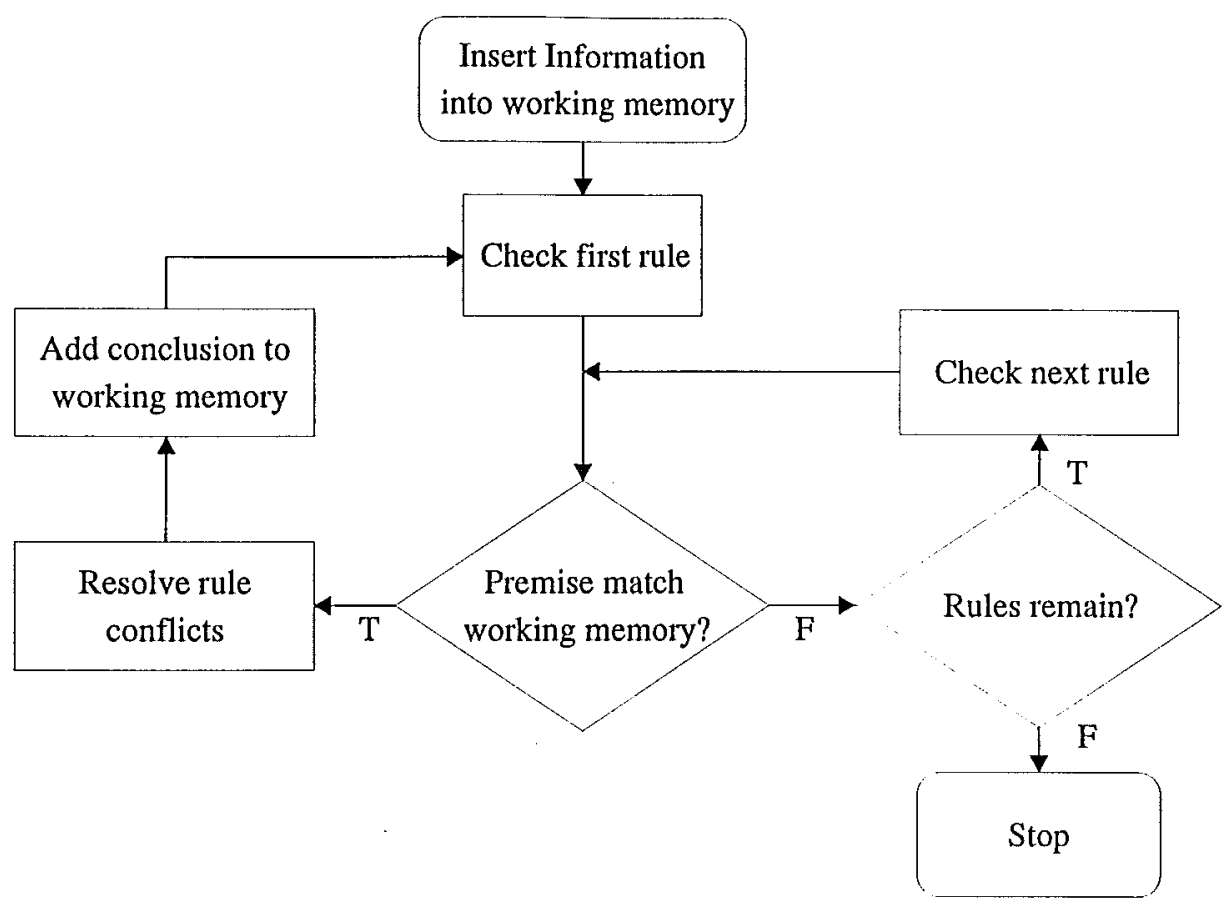

Figure 2.6 The Forward chaining inference process.

The process then repeats back to checking the first rule for a match given the new information in the working memory. If a match is not found at any stage then the next rule is checked for a match. If cycle repeats until there are no more rules left to fire. Using this method, a forward chaining process concludes everything possible from available information. This may not always be appropriate as unnecessary conclusions maybe drawn, however the inference engine has no way of knowing whether the information is useful or not.

Forward chaining can be viewed as a three step process:

1. Match or Recognise. Match all the rules against the working memory and determine which rules can fire.

2. Resolve Conflicts. If more than one rule can fire, choose the one to fire according to some strategy.

3. Act. Fire the rule and add the conclusion to the working memory.

When multiple rules are matched from the knowledge base, a strategy is needed to decide which rule to fire. Various strategies have been proposed. Some of these are:

- Fire the first rule that matches.

- Don't fire a rule which has already been fired. This can be used to prevent possible looping the inference process. This strategy may be used by other strategies. 
- Fire all matching rules then add all the new facts into the working memory.

- Fire the matching rules in sequence so that a new fact can be used when matching rules further down the sequence.

- Fire the most specific rule. That is fire the rule which has the least number of arguments or premises.

- Fire the highest priority rule. This can be used to place emphasis on more important rules.

\section{Backward chaining}

Backward chaining approaches the inference process from a completely opposite direction to that of forward chaining. Whereas forward chaining starts with information which is used to infer a solution or goal, backward chaining starts with a goal and then tries to find information to prove or support the goal. Durkin's definition is 'Inference strategy that attempts to prove a hypothesis by gathering supporting information' (Durkin, 1994, Ch. 4). The backward chaining approach is described in Figure 2.7.

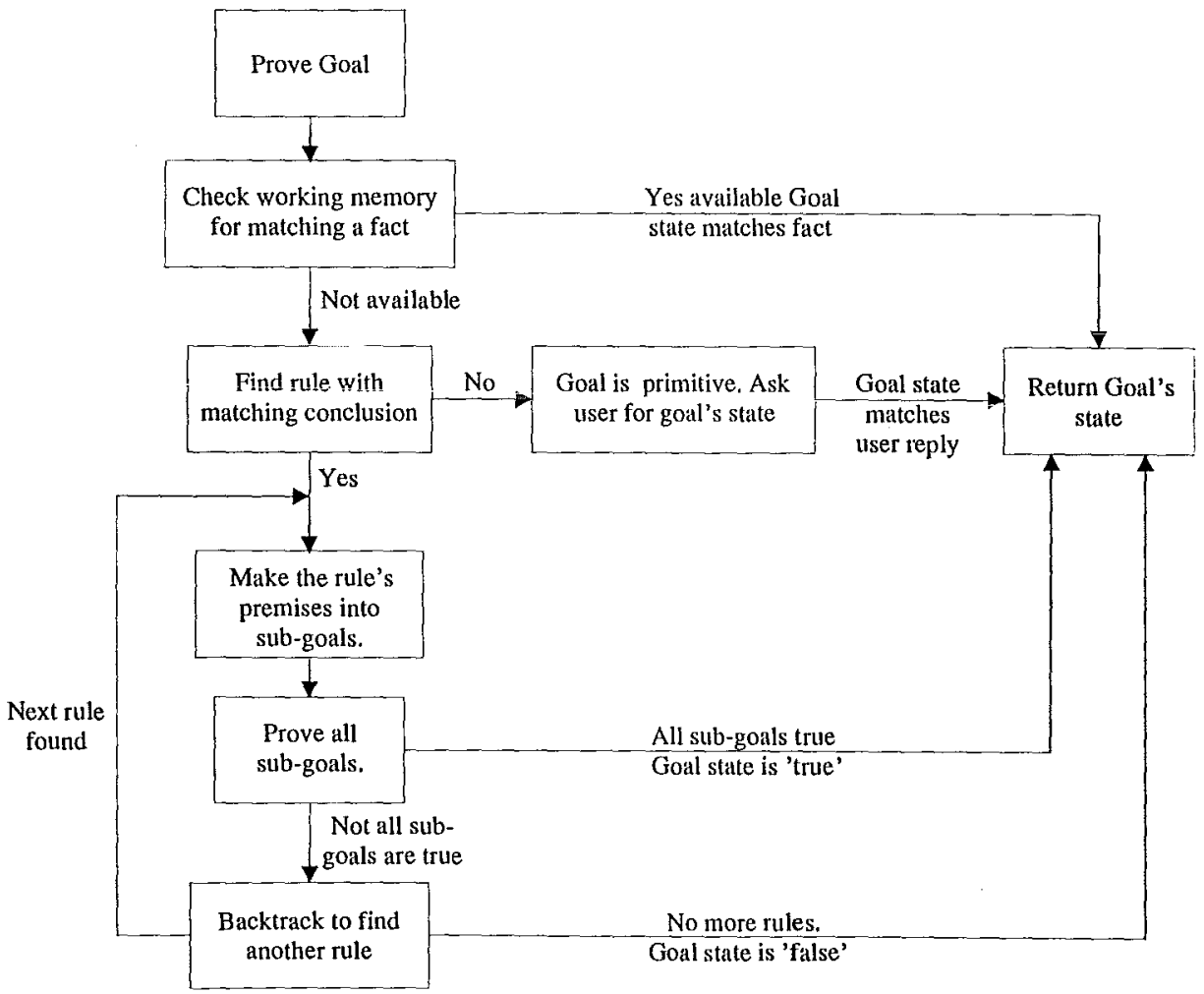

Figure 2.7 The Backward chaining process.

This process operates recursively in order to prove the goal in question. The steps involved in the process are: 
- The working memory is checked to see if there is a fact which matches the goal. The existence of such a fact immediately proves or disproves the goal and the process returns.

- The knowledge base is then searched for a rule with a matching conclusion. If no such rule exists then the goal is termed 'primitive'. This simply means that the solution to the goal is not determined by a rule, rather the solution is obtained by asking the user for the state of the goal. Once the user has answered then the process returns.

- If a matching rule is found then proving each premise in the rule will prove the rule. The rule's premises then become sub-goals of the current goal.

- The sub goals of the current goal are then proved in the same fashion as the current goal. This is performed by calling the backward chaining process for each sub goal. If all the sub-goals are proven 'true' then the rule and consequently the goal are also proven true, and the process returns.

- If not all the sub-goals are proven 'true', then the process backtracks to find another rule from the knowledge base. This next rule will be used to try and prove the goal. If there are no more rules in the knowledge base then the goal can not be proven, so the process returns with a false state.

The process does not end until the goal at the top level of the recursion is processed.

\section{Combined Approach}

Both of the forward and backward chaining techniques have their advantages and disadvantages. These are outlined in Table 2.2

As both methods have there advantages and disadvantages, the use of which method depends mainly on the problem to be solved. The simplest way to choose which method to use is to think of how a human expert would solve the problem.

Another alternative is to use both methods to solve the problem. This is also a common approach used by human experts. For example in a diagnosis problem, the human expert initially gathers information and infers a set of possible hypothesis. Each hypothesis is then tested by gathering supporting information. So this combines forward chaining for the first task with backward chaining for the second. This is called the separate system approach. It can be further expanded by using meta-rules in the forward chaining process which fire on a certain conditions. The meta-rules can then start a backwards chaining process to verify the condition. Once the condition has been verified, then forward chaining process can resume. 


\begin{tabular}{|c|c|c|}
\hline Method & Advantages & Disadvantages \\
\hline Forward & $\begin{array}{l}\text { - Good for problems which begin } \\
\text { with data gathering and then infer } \\
\text { from the data. } \\
\text { - Can provide a large amount of } \\
\text { new data from a small initial set } \\
\text { of data. } \\
\text { - Good for planning, monitoring, } \\
\text { control and interpretation tasks. }\end{array}$ & $\begin{array}{l}\text { - No means for differentiating im- } \\
\text { portant data from less important } \\
\text { data. } \\
\text { - The system will ask all possi- } \\
\text { ble questions even when only a few } \\
\text { questions are needed for a conclu- } \\
\text { sion. } \\
\text { - Totally unrelated questions may } \\
\text { be asked. }\end{array}$ \\
\hline Backward & $\begin{array}{l}\text { - Good for problems which proves } \\
\text { a hypothesis. } \\
\text { - Questioning remains focused on } \\
\text { a given goal. } \\
\text { - Only searches for data relevant } \\
\text { to the current problem. } \\
\text { - Good for diagnostic, prescrip- } \\
\text { tion and debugging tasks. }\end{array}$ & $\begin{array}{l}\text { - Often will continue to follow a } \\
\text { given line of reasoning, even when } \\
\text { it is no longer valid. }\end{array}$ \\
\hline
\end{tabular}

\section{Searching}

Searching is an important area of problem solving using expert systems. Typically a backward chaining problem can be represented graphically by a tree structure. This is normally known as the inference tree or the problem space. Within this inference tree may exist any number of solution or goal nodes. To solve the problem represented by the tree, one of these solution nodes must be found by searching the tree. Further solution nodes can be found by backtracking within the tree until all the solutions are found or the tree has been exhaustively searched. For example, say that Figure 2.8 contains a problem tree with a number of nodes labelled from a to 1 and two solution nodes $f$ and $j$.

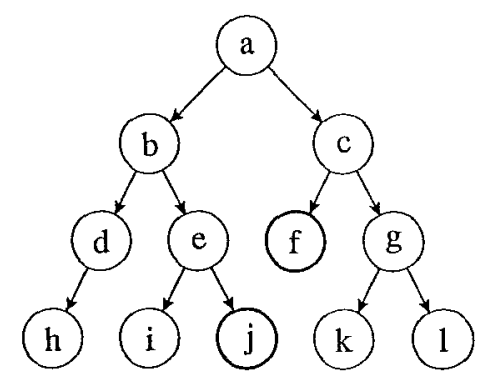

Figure 2.8 An example problem tree with two solution nodes.

There are a number of methods for searching a problem tree. Two of these are the 
depth-first and breadth-first. A depth first search looks for a solution down a path until a solution or a dead end is found. If the dead-end is found then the search backtracks and goes down another path. This continues until a solution node is found or the tree has been exhaustively searched. The depth first search lends itself to a recursive approach. This is shown by the following psuedo-Prolog description of a recursive depth-first procedure (Bratko, 1990, Ch. 11),

To find a solution path, Sol, from a given node, $\mathbf{N}$, to some goal node:

1. if $\mathrm{N}$ is a goal node then $\mathrm{Sol}=[\mathrm{N}]$

2. if there is a child node, $N 1$, of $N$, such that there is a path Sol1 from N1 to a goal node, then Sol $=[\mathbf{N}-\mathrm{Sol}]$

Figure 2.9 shows how the depth-first search visits the nodes in the example.

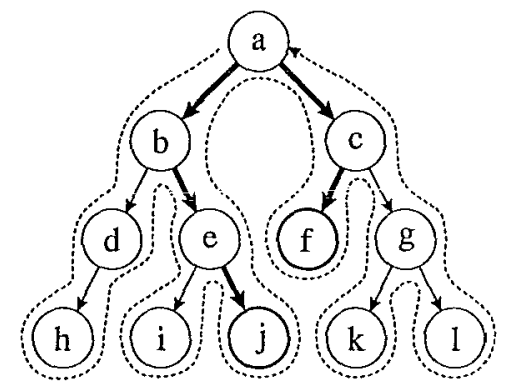

Figure 2.9 The tree example showing the depth-first search path.

In this example, the solution $[\mathbf{a}, \mathbf{b}, \mathbf{e}, \mathbf{j}]$ would be found first. Further backtracking would find the second solution $[\mathbf{a}, \mathbf{c}, \mathbf{f}]$.

A breadth-first looks for a solution of along all the nodes on one level of the problem tree before processing those nodes at a lower level. The description of the search method is (Bratko, 1990, Ch. 11),

To do the breadth-first search when given a set of candidate paths:

1. If the first path contains a goal node as its head then this is a solution of the problem.

2. Otherwise remove the first path from the candidate set and generate the set of all possible one-step extensions of this path, adding this set of extensions at the end of the candidate set, and execute breadth-first search on this updated set.

This is shown in Figure 2.10. This shows that the shorter solution path, $[\mathbf{a}, \mathbf{c}, \mathbf{f}]$, is found before the longer solution, $[\mathbf{a}, \mathbf{b}, \mathbf{e}, \mathbf{j}]$.

Table 2.3 (Durkin, 1994, Ch. 4) describes some of the advantages and disadvantages between the depth-first and breadth-first methods. Both methods are guaranteed to 


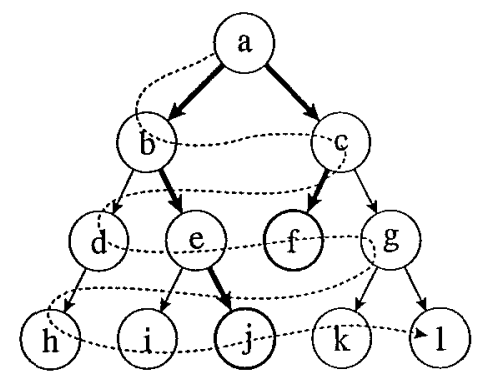

Figure 2.10 The tree example showing the breadth-first search path.

find a solution as they exhaustively search the problem space. However this makes them both inefficient when dealing with large problem trees.

Table 2.3 The advantages and disadvantages of the depth-first and breadth-first search techniques.

\begin{tabular}{|c|c|c|}
\hline Method & Advantages & Disadvantages \\
\hline Depth & $\begin{array}{l}\text { - If the solution is known to be } \\
\text { deep within the problem tree, this } \\
\text { will get to the solution without } \\
\text { wasting time looking for shallow } \\
\text { solutions. } \\
\text { - Maintains focus on the current } \\
\text { line of reasoning within the prob- } \\
\text { lem tree. In an expert system, this } \\
\text { would mean a sequence of ques- } \\
\text { tions would be related to a certain } \\
\text { issue. }\end{array}$ & $\begin{array}{l}\text { - Inefficient for shallow solutions. } \\
\text { May take a long time to find a shal- } \\
\text { low solution. It may unsuccessfully } \\
\text { search exhaustively a branch of the } \\
\text { tree before searching a branch with } \\
\text { a shallow solution. }\end{array}$ \\
\hline Breadth & $\begin{array}{l}\text { Does not miss easy solutions. } \\
\text { Shallow solutions within the prob- } \\
\text { lem tree will be found quickly and } \\
\text { efficiently. }\end{array}$ & $\begin{array}{l}\text { - Inefficient for deep solutions. For } \\
\text { solution located deep in the prob- } \\
\text { lem tree, this will take a long time } \\
\text { to find it. } \\
\text { - Provides poor focus on a line of } \\
\text { reasoning. Nodes are processed in } \\
\text { a seemingly unrelated order. }\end{array}$ \\
\hline
\end{tabular}

There are a number of methods to overcome some of these disadvantages. Depthfirst with iterative deepening combines the advantages of the depth-first and breadthfirst searches. A depth-bound is used to limit the depth of the search. Initially this bound is set to 1 , so the all the nodes to a depth of 1 are searched, as in the breadthfirst case. The bound is then increased, and the next two levels are searched and so on. This guarantees the that shallow solutions are found but is also efficient when the solutions are deep in the problem tree. The best-first search is known as an informed search unlike the three earlier uninformed searches. This method uses knowledge about the problem to guide the search. A heuristic is used to choose which goals are likely 
to lead to a solution. This approach mimics human reasoning closely, and when the heuristics are chosen well can lead to a very efficient search. However the method does not search the problem tree exhaustively, so there is no guarantee of finding a solution. 


\section{Chapter 3}

\section{QUEST DESIGN AND MODELING}

This chapter outlines the design concepts and justification used in the Quest project. The first section examines the questionnaire process and discusses the advantages and disadvantages of a computer-based approach. Section 3.2 examines the requirements of such an approach. Section 3.3 then presents a questionnaire structure model based around the explicit ordering of questions within a questionnaire. This approach is called the Question Order Flow model. Two variations of this model are presented along with the implementation and consequent conclusions about the approach. Finally section 3.4 presents the Required Information flow model which focuses on the information requirements of the questionnaire process.

\subsection{THE QUESTIONNAIRE PROCESS}

In chapter 2 , the steps in questionnaire development were identified. The sequence of four tasks in figure 2.1 with dark borders are the most suitable for computer aided assistance of some form:

1. Questionnaire construction. This task can benefit from a questionnaire editor program. Such a tool allows the author to design a questionnaire in a consistent manner.

2. Questionnaire pre-testing. This task will gain the least from direct computer assistance as it requires a group of respondents to trial the questionnaire. The respondent's replies are then analysed to determine if the questionnaire includes a balanced set of questions. If it does not then design phase must be reiterated in order to produce a balanced questionnaire. As the pre-testing is mini-questionnaire in itself, the tools used in the other three tasks can be of benefit here.

3. Questionnaire presentation. A computer based questionnaire presentation program is able to present a questionnaire in the same manner every time. This helps reduce any bias normally introduced by a human questionnaire supervisor. 
4. Questionnaire replies analysis. A computer tool is normally used to process the questionnaire replies once the replies were manually entered. A computer based analysis tool that has knowledge of the questionnaire format is able to directly access the saved replies from the questionnaire presentation program.

The prime function of a questionnaire is to retrieve meaningful information from a sample population. In order to achieve this, the questionnaire author first decides what information is required. Then the questions necessary to meet these requirements must be formulated. These questions can then be presented to the respondent. The respondent answers the questions as fully as possible. The information in the respondent's replies are then collated by the author. If the right questions were chosen by the author, the author now has the information required.

In general the author formulates the questionnaire by hand. The questionnaire is then prepared for the respondent to reply to the questions. This is done by clearly displaying the questionnaire on paper usually with the aid of a typewriter or word processor. The respondent will then take the formatted questionnaire and reply by answering either directly onto the questionnaire sheet or a specially prepared answer form. Once finished, the author then collates the replies by hand. The raw questionnaire data can then be interpreted by hand or computer to produce an overall result for each respondent's replies.

The actual processes of creating and preparing the questionnaire and then collating and manipulating the data can be a complicated task for the author. The respondent may find answering a detailed questionnaire difficult especially if the instructions are difficult. Often a questionnaire or survey needs a trained administrator to go through the questionnaire with the respondent. The tasks then of the author and the respondent both can involve large amounts of work.

A computer based tool can reduce the amount of work required by the author. This allows the author to more thoroughly test the questionnaire before presenting it to the target respondents. Alternatively the author could put more effort into producing an interactive questionnaire. An interactive questionnaire that provides feedback to the respondent may be more motivating than a plain paper questionnaire for the respondent.

Figure 3.1 shows a diagram of the questionnaire system with the use of a computer based tool. A computer based tool can aid the author with the questionnaire design and preparation processes. A computer program can then be used to display to questionnaire to the respondent and collate and store the respondent's replies. Finally another computer program can process and manipulate the replies from a number of respondents according to the author's wishes. The author can retrieve these processed results and draw conclusions from them. 


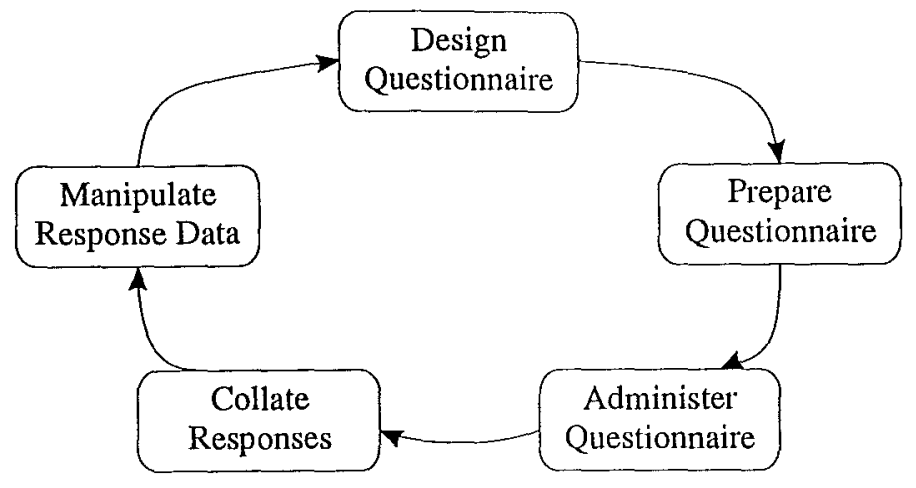

Figure 3.1 Stages in the questionnaire design process with a computer based tool.

Every questionnaire is made up of questions. An important problem is how the questions relate to each other. Related to this problem are decisions of when and why a question should be asked. When an author is obtaining information from a respondent, the respondent will be able to follow the author's line of questioning better if the questions asked are related in some way. For example, a number of random unrelated questions are likely to confuse the respondent. If a number of related questions are asked together, then it follows that those questions form a group or section of questions. Within each group of questions, there may be a number of questions which are not appropriate to ask depending on certain conditions. For example, if the reply to question A was 'yes', then ask question B otherwise skip forward to question C. Questions such as $B$ and $C$ are conditionally dependent on the replies of other questions. Obviously the conditionally dependent questions should be asked only if the necessary replics are available. Therefore an ordering is defined for groups of questions with these properties.

For each of the three main functions identified previously that a computer based system must handle (questionnaire preparation, questionnaire administration and summarising the results) a separate application would be logical. Each application would then communicate with each other through the use of files. A questionnaire file would contain a complete description of a questionnaire. The questionnaire file would be produced by the questionnaire editor application and be read by the questionnaire administrator application. The administrator application would produce a reply file which would be read by the summary application. Finally the summary application would store a number of reply files in a questionnaire database file. The questionnaire database file would hold a number of reply files from a specific questionnaire. The following section takes these observations of the questionnaire process and produces a list of requirements which a computer based questionnaire system should follow. 


\subsection{COMPUTER BASED QUESTIONNAIRE REQUIREMENTS}

The following two statements concisely describe the questionnaire process:

"A series of predefined questions are presented to a respondent. The respondent's replies are retrieved and stored. Once all the relevant questions have been presented, the replies are then summarised for further analysis."

"The major aim of a computer based questionnaire system is to maximise the information gained from a respondent population while minimising errors and bias."

These two statements imply a number of requirements for a computer based questionnaire system:

- The questionnaire author must be able to specify a series of questions and the ordering relationship between the questions.

- The author must be provided with a sufficient set of question types to be able to ask most questions.

- The ordering relationship between questions must allow the author to create complex questionnaires. This includes the ability to structure questions around the answers to other questions.

- The author must be able to specify a summary format which can be automatically generated from the respondent's replies to the questions.

- When presenting the questionnaire to the respondent, the system must be able to interpret the questionnaire structure utilising the respondent's replies when necessary.

- Each question must be presented to the respondent in a simple and obvious manner, and provide a mechanism to receive the respondent's reply.

- A replies storage facility is required to hold all the respondent's replies while the questionnaire is being administered to the respondent. Once the questionnaire has been presented to the respondent, a further means of permanently storing the respondent's replies is necessary.

- A facility must be available to analyse a series of summaries of the respondents' replies in order to provide statistics over the whole respondent population. 
An expert systems approach would seem appropriate for a computer based questionnaire system. If the problem in this case is considered as questionnaire administration, then the definition of an expert system from section 2.2 can be paraphrased as "a system which is capable of administering a questionnaire using a computer model of a questionnaire process, then reaching the same conclusions that a human expert would reach given the same questionnaire and respondent". Section 2.2.1 gives two general reasons why expert systems are used. In terms of replacing a human expert, a computer based questionnaire system would be beneficial for the following reasons:

1. The expertise encoded into the questionnaire's structure would be available at all times of the day and at different locations. This is possible because the questionnaire file could be cheaply copied and distributed to various locations.

2. It would automate the task of administering the questionnaire. This would guarantee consistent quality of the questionnaire presentation every time the questionnaire was administered.

3. The cost of training a new computer operator for the system would be minimal compared to the training cost for an adequately trained human questionnaire administrator.

In the case of a general medical practice, a computer based health screening questionnaire could provide the general practitioner with useful information about the patient before the patient sees the doctor. The computer based system can assist and improve the productivity of the human expert, namely the doctor, by performing an initial assessment of the patient. The doctor is able to quickly assess the accuracy of the assessment and make a decision once the recommendations have been verified.

The most important factor in a computer based questionnaire system is the method used to represent the questionnaire structure. The method used will dictate the strategy used to interpret the questionnaire structure. Section 2.2.4 indicates that a forward chaining approach would not be useful because of the following disadvantages:

- Related questions would have to be placed on the same level within the questionnaire tree structure. This is counter-intuitive as people tend to group related questions and information down the same branch in the tree. In other words, people form hierarchical trees of information. With a forward chaining system, organising conditional dependencies between questions on the same level within the questionnaire tree structure would be difficult. This would also affect questions on the lower levels of the structure which would be subject to the same dependencies as those in higher levels.

- Important questions should have the greatest significance when deciding which questions are asked next. So the important questions should be asked first. In 
a forward chaining system, asking the questions in any order will produce the same result. This is because the rules in such a system are only fired after the questioning process has finished.

- All questions within the questionnaire will be asked. From this the inference process would form conclusions about the respondent. This is a very general approach which is not suitable for proving or disproving a hypothesis. The first item in the list in section 2.1 mentions that one of the first tasks in questionnaire design is to decide on the hypothesis to be investigated. The hypothesis may only take one question to be answered or it may require all the questions, either way only the relevant questions should be asked.

A backward chaining system would not suffer these three deficiencies primarily because it uses a hierarchical tree style arrangement for the questionnaire tree structure. Firstly with a hierarchical tree structure, related questions would be arranged into the same branch within the tree. Secondly the most important questions can be placed towards the top of the hierarchy, and so would be asked before questions lower down. Thirdly, the more important questions could be used to dictate which questions further down the branch are asked or skipped.

The main disadvantage of a backward chaining system is that it may continue to follow a line of reason which is no longer valid. In the case of a questionnaire, this is not acceptable. Consider a branch in which the first question is "Are you female?". Questions lower in the branch may ask questions about feminine issues which would not be appropriate for a male respondent. Obviously some control over this situation is necessary, and as these types of question-skipping patterns are an integral part of the questionnaire design it should be the questionnaire author who controls them. So a facility for implementing skipping patterns must be built into the questionnaire representation structure.

A computer based questionnaire authoring tool at the most basic level will facilitate in the preparation of a questionnaire. It will be able to provide a set of primitive questionnaire manipulation functions. Examples of these functions may be Create Question, Delete Question, Insert Question, Move Question, Edit Question. Using these functions, an author will be able to prepare a questionnaire. However the whole task of producing a questionnaire is a case of Knowledge Acquisition. The information needed to build a questionnaire must be acquired from the author. An authoring tool must be intuitive for the author to use as it is effectively taking the place of a knowledge engineer. Normally a knowledge engineer would obtain the information from the human expert and incorporate the knowledge into the expert system. However in this case the questionnaire author and the questionnaire authoring tool must both make up for the absence of the knowledge engineer. Such a tool needs to be capable of editing all facets 
of a questionnaire. This includes the questionnaire structure, content, appearance and output.

Section 2.1.1 indicates that two of the disadvantages of computer based questionnaires are computer illiteracy and a lack of rapport between the respondent and the computer questionnaire administrator. These problems reflect on the quality of information the respondent gives in response to the questions because of possible respondent bias and/or errors. As one of the main requirements is to minimise error and maximise information during questionnaire administration, it is important to provide a suitable environment for the respondent. The computer based questionnaire's user interface must be simple, intuitive and easy to use. The lack of rapport can be compensated for by utilising multimedia displays and feeding extra information back to the respondent.

It is most important that the results of the questionnaire session can be easily extracted from the respondent's replies. Gaining suitable information from the respondent's replies is the whole purpose of implementing a questionnaire. However the raw replies will not always tell enough to evaluate the questionnaire's hypothesis. The replies must be collated, verified and analysed before any judgements can be made. This type of work can be performed very well by a number of computer applications; however the data often has to be entered manually. A computer based questionnaire system has the advantage that the respondent's replies have already been stored. However, the raw responses are not always stored in a convenient form. The replies would be much more useful if they could be summarised by the questionnaire program. This summary would be a report of the respondent's replies and any useful information derived from them. As the author has designed the questionnaire they should be able to design suitable summaries, one for informing the respondent, and one for the author.

Summaries are useful for processing each respondent's replies, however it would be advantageous to analyse a series of respondent's replies statistically. While there are a number of well established statistical programs available it would be worthwhile including a simple statistical capability which is tuned to questionnaire analysis. For further processing the respondent's replies could be exported to a dedicated statistics package.

To sum up, the following list outlines the requirements for a computer based questionnaire system:

- Flexible questionnaire structure model.

- Scheme for interpreting the questionnaire structure model.

- An questionnaire editing interface which allow manipulation of the questionnaire structure, appearance and summary facilities.

- Simple and flexible user interface between the questionnaire administrator and the respondent. 
- Scheme for summarising and storing the respondent's replies.

- Facility for analysing the respondent's replies and summary in order to produce statistics useful in evaluating the survey hypothesis.

The following two sections outline two different methods for representing a questionnaire in terms of the above requirements. The Question Flow Model defines the questionnaire as a hierarchical flow chart type structure. The Required Information Model approaches the questionnaire as a tree of information driven goals.

\subsection{QUESTION ORDER FLOW APPROACH}

The Question Flow Model was the model originally designed for the questionnaire system. It is based on the idea that a questionnaire is a group of ordered questions. These questions are arranged in a flow chart style graph pattern. This style of questionnaire representation went through three revisions before a number of limitations in the model made it necessary to try another approach. The three revisions are:

1. The LifeQuest medical questionnaire. This is the original questionnaire model developed by Dave Powley, Steve Penno, Scott Marshall, and Alan Simmons in 1992 under the supervision of Dr Chris Carey-Smith. This model described the questionnaire using a series of Prolog internal databases, each database residing in its own file. The questionnaire content was fixed as a health screening questionnaire due to the requirements of the project and the format of the internal databases.

2. The QUEST Qucstionnaire System Version 1. This was the system developed by Tim Russ and the author in 1993. The questionnaire model was largely refined by incorporating all the internal database files into a single database file. The database formats were expanded to store extra information about the actual questionnaire and to accommodate new facilities such as multimedia. This generalised the model and allowed new questionnaires to be implemented on the system.

3. The QUEST Questionnaire System Version 2. This system was the last revision of this approach. Again it expanded the scope of the questionnaire model to improve the model's flexibility and useability. This model also included provision for programmable summary and user interface capabilities. A review of the model was carried out in 1994 and a number of limitations of the model were outlined. After these limitations were examined, a number of the limitations proved prohibitively difficult to resolve satisfactorily. Consequently the model was abandoned for a more flexible approach (see section 3.4). 
The following sub sections outline the questionnaire model, the interpretation of the model, the implications of the model for the author and respondent, the implementations of the model and the conclusion drawn about the model.

\subsubsection{The Questionnaire Structure Model}

Two models for the question order flow approach are outlined here. The first is from version 1 of the Quest Questionnaire System and is referred to as the standard question order flow model. The second model is from the second version of the Quest system and is referred to as the extended question order flow model. In both cases the information encoded by each model can be though of as structural knowledge of the questionnaire as described in section 2.2.3. The actual knowledge representation scheme used is a combination of both the network and structured methods. This is because the questionnaire structure is stored as a tree graph with the arcs between the tree nodes indicating an ordering and information about each node is stored as attribute slots within an object.

\section{Standard Question Order Flow Model}

As the name of this model implies it is structured around the ordering of a set of questions. As one of the requirements mentioned in section 3.2 , this model must be able to implement question skipping patterns. This is implemented with the concepts of blocks and links.

- Blocks. A block is the main functional element in this model. For example each question in the questionnaire is a question block. Each block has the following fields:

- BlockId. Uniquely identifies the block.

- Question Text. The actual question to ask the respondent.

- Replies List. A list of text strings. The number of list items determines what kind of question is asked.

- Link Conditions. A list of link condition items. Each item in the list must prove true before the following block can be executed.

- Picture. A filename of a picture file to display with the question.

- Help Text. Text string which gives information on how to answer the question.

- Why Text. Text string which describes why the question is being asked.

- Links. A link is used to describe the ordering of the blocks. The ordering of the blocks is done by specifying a parent block and a child block. Once the parent 
block has been executed, each link with a matching parent block field indicates one possible child block to process.

When a block is processed, the question it represents is shown to the respondent using the information in the block's fields. When the respondent has answered the question, the reply is stored along with the associated BlockId field in a replies database. These replies can be used when the questionnaire inference engine processes the questionnaire structure.

In order to facilitate a wide range of questionnaires, a suitable range of question types are necessary. To meet these requirements the following question types were devised (section 2.1.3 describes the open and closed question types):

- Text Entry. This is an open question type. A question is asked of the respondent, and the respondent replies by entering a text response using the keyboard.

- Single Select List. This is a rating type of closed question. The respondent replies to a question by selecting a single item from a predefined list of responses.

- Multiple Select List. This is an inventory type of closed question. The respondent replies to a question by selecting any number of items from a predefined list of responses.

- Information. This is not a question but a means of providing feedback to the respondent.

Each of the blocks could potentially evoke a response from the respondent. This reply is in the form of a text list. Consequently the link conditions were based around a text string reply to a previous block in the questionnaire. This is discussed further below.

Figure 3.2 shows a simple example that includes a number of blocks and links. Table 3.1 lists the blocks and links involved in this example. Each block entry in the table lists the BlockId, Link Condition fields and the block type.

Table 3.1 The list of blocks and links within the example.

\begin{tabular}{l|l}
$\operatorname{Blocks}$ & Links \\
\hline $\operatorname{block}(1,[])$ & question_space $(1,100)$ \\
$\operatorname{block}(100,[])$ & question_space $(1,200)$ \\
$\operatorname{block}(101,[])$ & question_space $(100,101)$ \\
$\operatorname{block}(102,[])$ & question_space $(101,102)$ \\
$\operatorname{block}(103,[])$ & question_space $(102,103)$ \\
$\operatorname{block}(200,[])$ & question_space $(200,201)$ \\
$\operatorname{block}(201,[])$ & question_space $(201,202)$ \\
$\operatorname{block}(202,[1 \operatorname{link}(201$, "Yes")]) & question_space $(201,204)$ \\
$\operatorname{block}(203,[])$ & question_space $(202,203)$ \\
$\operatorname{block}(204,[\operatorname{link}(201$, "No")]) &
\end{tabular}




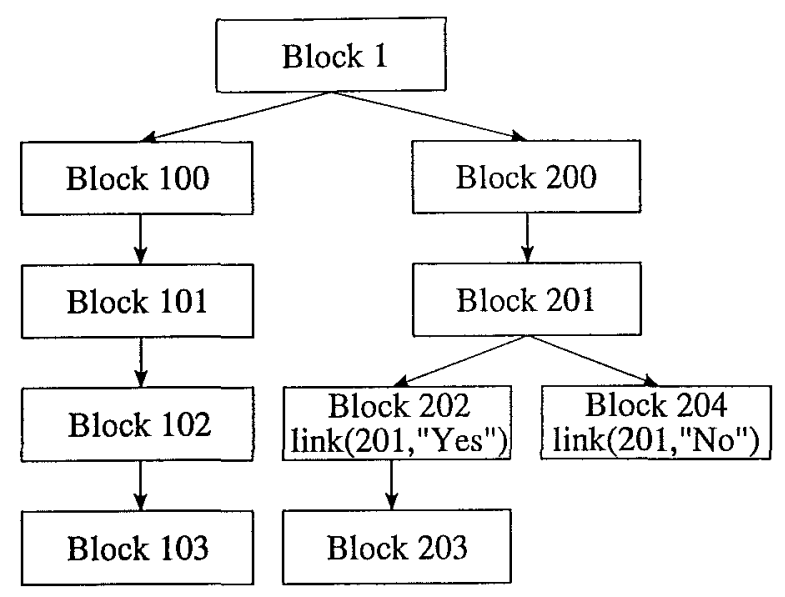

Figure 3.2 Example of the Block and Link concepts.

The figure also introduces the concept of sections of questions and section introductions. It is obvious that there are two main branches within the tree structure and the numbering of the blocks reflects this. Blocks 100 to 103 form one section of questions and Blocks 200 to 204 form another. Since, when asking a group of questions, it makes sense to introduce the questions to the respondent, an introduction block type is defined to provide such an introduction. Blocks 1, 100 and 200 would all be introduction blocks. By definition block 1 is always the questionnaire introduction block, block 100 is the section introduction block for blocks 101 to 103 . Likewise block 200 is the section introduction block for blocks 201 to 204 .

The use of link conditions introduces the concept of 'OR' branching within the questionnaire structure. Question skipping patterns are implemented through the use of these OR branches. The split between blocks 202 and 204 represent an OR branch. This is because the flow of the questionnaire would either go down one branch or the other branch. The link conditions for each question determines which branch is followed. This is discussed further in section 3.3.1.

In general the flow of a questionnaire is from top to the bottom. If a branch is taken, then the flow continues down until the bottom of that branch is found, at which time the questionnaire has ended. However this does not work well for sections of questions. Each section of questions should be asked one after another without any conditions attached. So the sections of questions are separated into 'sub-questionnaires'. The sections are then arranged in order in a separate but simpler tree. Within this top level questionnaire tree, section blocks are arranged through 'AND' branching. This is because the first section and the second section and the third section and so on, are processed. Figure 3.3 shows this situation. So a two-level hierarchy defines the separation between the questionnaire level which contains the sections and the section level which contains the questions. 


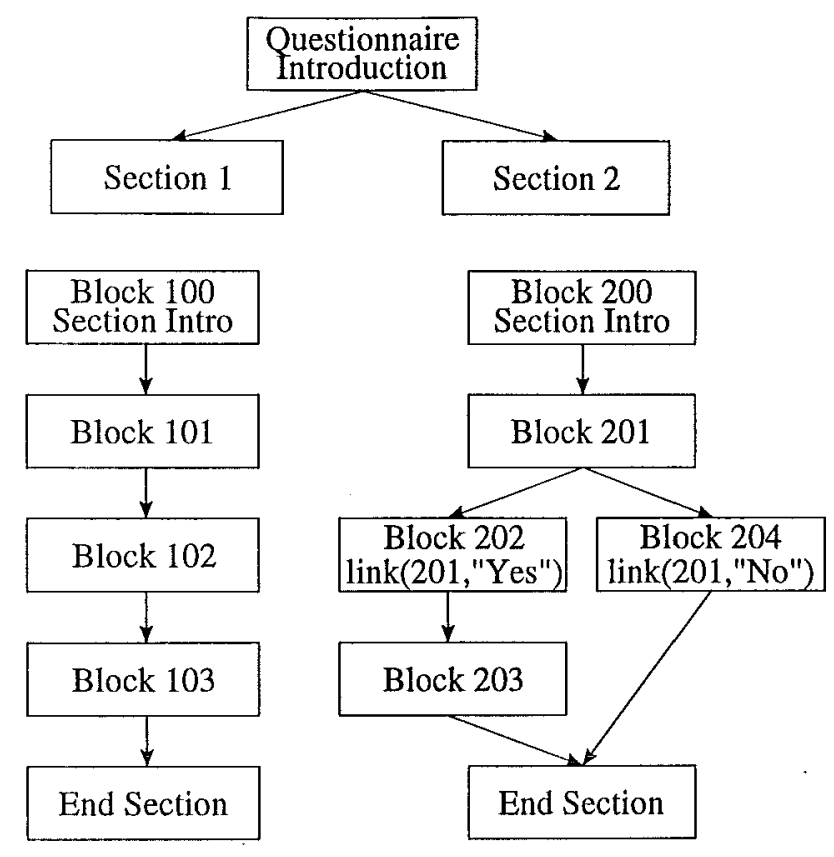

Figure 3.3 Example of the Section concept.

The summary and summary analysis requirements outlined in the previous section were not explored at this stage. However a scoring system for the LifeQuest program was designed by Scott Marshall in 1992. This scoring system was strongly linked to the LifeQuest questionnaire content, and no effort was made to generalise it to the same level as the authoring and administering aspects of the updated Quest system.

\section{Questionnaire Model Interpretation}

Given the questionnaire structure model, the means for interpretation is basically a modified depth-first search. This search is based around three predicate databases: the question_space database which describes the question order relationships, the blocks database which contains the details of each block, and the replies database which stores the respondent's replies to each question.

The questionnaire structure is interpreted using a backward chaining algorithm to implement a depth-first search. The goal in this search is simply the last block in a branch. Once this block has been reached then that section of questionnaires has been processed. For example in figure 2.8 (see page 31) node a would be the start of the section and nodes $\mathrm{h}, \mathrm{i}, \mathrm{j}, \mathrm{k}$ and $\mathrm{l}$ are all valid goals of the depth-first search. Figure 3.4 contains a simple version of this algorithm which is very similar to the algorithm which appeared in the LifeQuest version of this model.

Line 1 is the head of the predicate and takes the argument BlockId. In line 2 a match is sort for BlockId in the question_space database, if no match is found then there are no more questions to ask within this section. This predicate will fail and will 
Figure 3.4 The basic depth-first search algorithm.

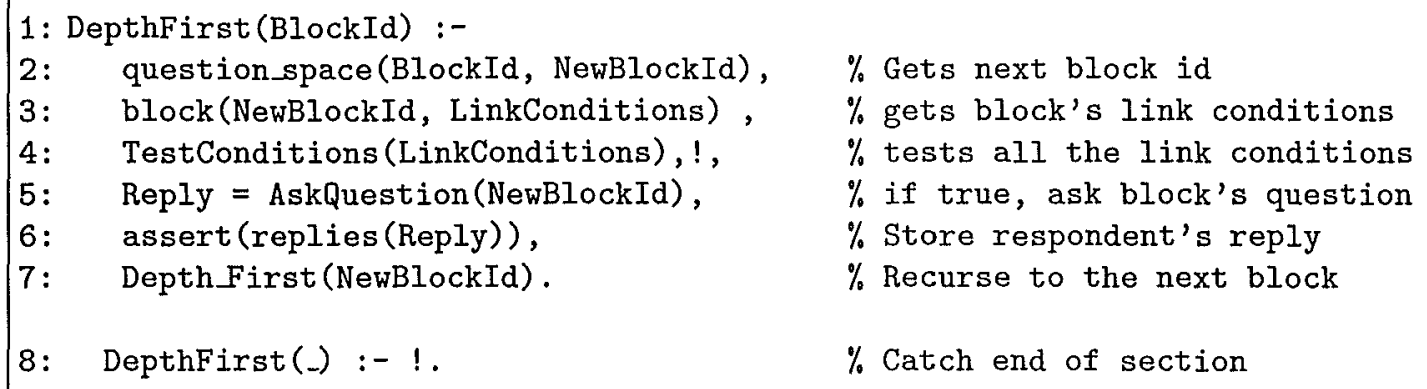

backtrack to the next clause which is in line 8. This clause catches the fail and succeeds at the end of a section. Otherwise the match will have obtained the block identifier of the next block in the section NewBlockId. In line 3 the link conditions for this block are obtained from the block database. These link conditions are tested in line 4 by the TestConditions predicate. This predicate will fail if none of the conditions evaluate true and the algorithm will backtrack to line 2 in order to obtain the next block which follows BIockId. If the predicate succeeds then the question associated with that block is asked of the respondent, and the reply is saved in lines 5 and 6 . Line 7 then calls the head of the predicate with the next block identifier as the parameter in a recursive fashion. This continues until the end of a branch is reached.

The TestConditions predicate makes use of the replies database to check the state of the conditions. The LinkConditions list consists of links or references to the replies of other blocks. Each link is checked against the corresponding reply in the replies database. If the corresponding reply matches then link reply the TestLinkConditions predicate succeeds.

The Askquestion(BlockId) predicate is responsible for displaying the question to the respondent. The respondent's reply is also retrieved by this predicate and is returned so it can be saved in the replies database.

Typically an even simpler version of this algorithm will be used to implement the simple AND branching that controls the order in which the sections are presented.

\section{Extended Question Order Flow Model}

The Extended Question Order Flow model is an extended version of the standard question order flow model. The first main extension addresses the information that each block can return from the respondent after a question is asked. The previous model simply returned a text list. This has been expanded to returning three different reply list types, these are:

1. Text List. Returns a list of text strings. 
2. Index List. Returns a list of indices which correspondent to the index to a list based question type.

3. Variable List. Returns a list of real numbers.

The relevance of these becomes apparent with the new block types. There are two new block types, however each of the existing block types benefit from the enhanced reply types. All of the blocks are described here:

1. Text Entry. As before, simply returns the text reply.

2. Numeric Entry. The respondent must enter a number as a response. The text representation of the reply is stored in the text list, the integer representation in the index list and the real representation in the variable list.

3. Single Select List. A real value may be associated with each item in the predefined list of responses. The respondent does not see this value, only the predefined items. When a selection has been made, the text representation of the chosen item is stored in the text list, the index of the item in the index list and the associated value in the variable list.

4. Multiple Select List. As for the Single Select List question, except that the respondent can select any number of the predefined items. Note that this is the only question block type that can generate multiple entries in the reply lists. All the other question blocks generate single entries in the reply lists.

5. Information. Provides some feedback to the respondent. This block generates an empty list for each of the reply lists.

6. Calculation. This block can evaluate an arithmetic, relational or logical expression. The results of the evaluation are stored within the reply list in the same manner as the other questions. It is possible to reference any other block reply within the expression in order to perform a calculation on a number of respondent entries. Each reference contains an indicator to the type of referenced reply. For example, the calculation ' $\mathrm{g}(4, \mathrm{v})+\mathrm{g}(5, \mathrm{v})$ ' means add the variable list of block 4 to the variable list of block 5 .

The link conditions in the standard model were a property of the block. The link just provided a simple connection between the blocks. However in terms of a flow orientated model, the link condition property made better sense as a property of the link itself. In this way the questionnaire flows down a path or link if the conditions for that link are meet. Also since the reply type has been expanded to three types, each link condition should explicitly refer to the type of the referenced block in the same manner as the Calculation block. 
The standard model provides a two level hierarchy for the questionnaire level, which contains the ordered sections, and the section level which contains the questions. The extended model retains this two level structure, however with a couple of modifications. Instead of the limited AND branching in the questionnaire level, this is expanded to allow OR branching in the same fashion as the section level. This allows conditional links within this top level. For example a section or group of sections can now be bypassed or skipped through the use of an OR branch.

A further extension of the AND branching was considered for the section level. This form of AND branch is used to implement branching based on a reply to a multiple select list question. Currently a link condition can contain a reference to a multiple select list reply. However if a multiple select question contains a number of predefined items, it is difficult to create a tree structure which has a link associated for each item. For example, say a question is asked about family medical history "Have any of the following problems worried your family in the last year?" and the predefined replies are: Chest pain, Depression, Excessive thirst, and Wheeziness. For each of these replies a information block should be displayed giving more information on the problem. The extended AND branch allows this type of structure to be easily set up. This situation is shown in Figure 3.5 .

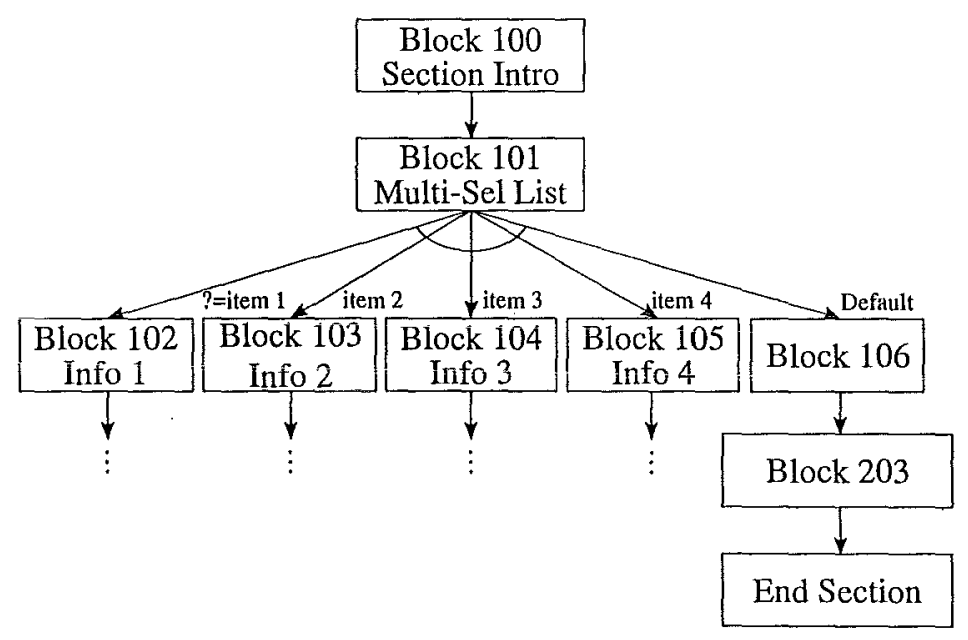

Figure 3.5 Example of the extended 'AND' branch.

Figure 3.6 shows the complexity involved in implementing this type of structure using the standard model. This figure does not show any of the link conditions and only includes three information blocks as opposed to the four in the above example. While not impossible to do in the standard model it would be complicated and error-prone.

With the growing complexity of the model it was decided to give some protection to the author when creating link conditions. A problem of ensuring "questionnaire consistency" was identified as an area worth investigation. Questionnaire consistency means that all the references made in the questionnaire are valid logically and also in 


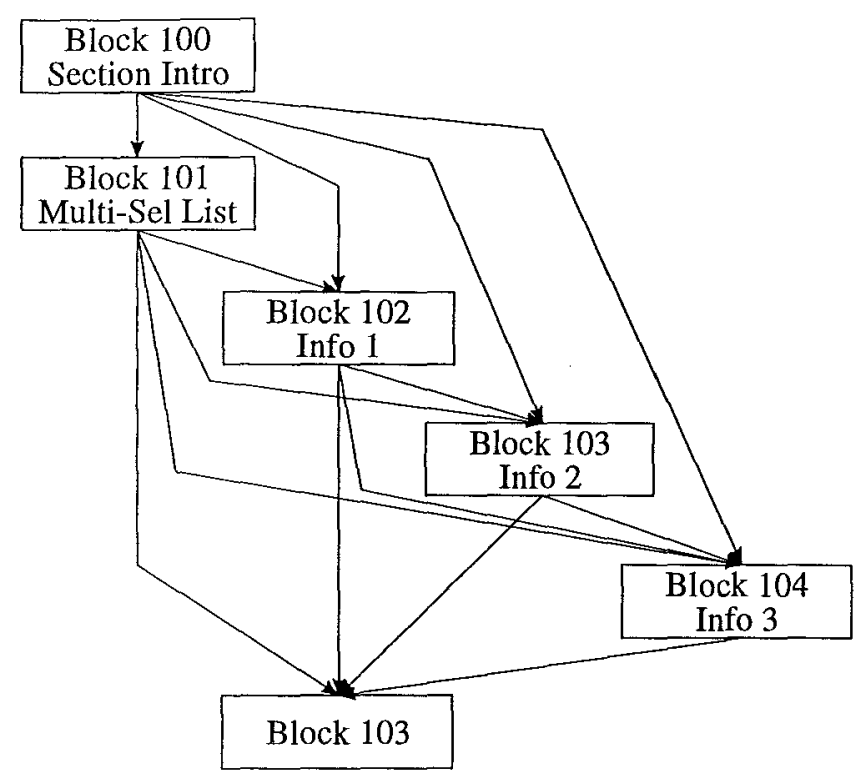

Figure 3.6 A simplified extended 'AND' branch example implemented using 'OR' branches.

context. A consistent questionnaire can be interpreted as intended by the inference process without error. However ensuring a questionnaire is consistent is a difficult process. This is discussed further in the next section.

\subsubsection{Implications for the Author}

The author's involvement in the system model is a complex one as the author is assumed to perform several tasks. These tasks are questionnaire design, questionnaire preparation and response analysis. It is assumed that the author wants to know certain information about a group of respondents. Therefore the author is responsible for producing a questionnaire and gathering the response information later. Once the information is analysed, the author now has the information desired.

An important factor in this process is the author's understanding of the questionnaire model. If the model is complex then the author will find it difficult to produce a questionnaire. However a simple questionnaire model may require more effort when producing a complex questionnaire. The concept of a flow-chart graph is familiar to most people. It shows the relationships between a number of tasks or functions in a simple order-related fashion. This concept is easily understandable and can be learnt quickly.

The concept of skipping patterns is also familiar to most people. Most paper questionnaires use skipping patterns of some form. The merging of the concepts of flow-chart graph and skipping patterns introduces new complications for the author. With the question order flow model, it is the author's responsibility to ensure that the skipping patterns are correctly implemented. The skipping patterns in this model are 
controlled through the use of the link conditions. A link condition is associated with a link between two blocks. The link condition determines if the link between these blocks should be followed, i.e. the second block is processed after the first block if the condition is met. Each link condition contains a reference to another block's reply. In order for the link condition to be evaluated, the referenced block must have already been processed. If this not the case then the questionnaire is inconsistent.

There are a number of possible types of questionnaire inconsistencies:

- In Links. As described in the paragraph above. A link condition has a reference to another block. If this block is deleted or moved ahead of the link condition then a link inconsistency occurs.

- In Calculations. Calculations will normally contain references to other blocks in a similar fashion to the link conditions. A Calculation inconsistency will occur if the referenced block is deleted or moved ahead of the calculation.

- Down a Branch. Conditional inconsistencies occur when there is more than one way to reach a block or link. If a link condition references a block which is down one side of a 'OR' branch, then it is possible that the other branch could have been taken to reach the current link. In this case the link condition does not have the required information to evaluate the condition.

These inconsistencies will occur either when the author is initially entering the questionnaire for the first time or when the questionnaire is being revised at a later date. It is expected that most of the inconsistency problems will occur during the revision phase. During this revision phase the questionnaire will be updated to fit the changing requirements of the author. It is more likcly that the author will make more errors during this phase as simple changes made may effect large parts of the questionnaire. The author could manually check the questionnaire for inconsistencies once the changes have been made. However it is possible that fatal inconsistencies remain which effect the correct flow and logic of the questionnaire. Hence there is a need for a consistency checker.

If the author has trouble learning the questionnaire model or has only just started learning, then they will probably make more mistakes. At this stage a questionnaire 'wizard' or 'expert' tool may be of some help to the author. A questionnaire wizard is a small program that talks the author through the process of creating a simple questionnaire. It does this by presenting a series of dialog boxes each containing questions on various aspects of the questionnaire design. Generally the information entered in the initial dialog boxes determine the type and number of questions later on. A simple sequence of questions would be:

1. General Questionnaire properties. Ask for the type of questionnaire and whether to automatically generate a summary. 
2. Questionnaire Introduction. Ask for the title and introduction of the questionnaire.

3. Sections Setup. Ask for the total number of sections within the questionnaire.

4. For the number of section entered, ask for the section name and section introduction.

Such a procedure would be able to create a simple skeleton structure of a questionnaire, but with no questions. Extending this procedure to create a number of questions is more difficult and would make for a longwinded process for the author. At this stage it would be better to have an 'insert question block' wizard. This could ask more localised questions about a smaller section of the questionnaire and then create a small block of questions within a section. Another alternative is to have a set of questionnaire templates. These templates are blank questionnaire structures designed with specific applications in mind. The author could load the template questionnaire closest to their needs. They would then fill in the blank questions and add or remove any extra questions.

\subsubsection{Implications for the Respondent}

The implications for the respondent are straightforward. The requirement that there be a "Simple and flexible user interface between the questionnaire administrator and the respondent" insists on this. The respondent must simply be able to comprehend the presented information or question and then respond in the appropriate manner. When presenting the information, there should be as little distraction to the respondent as possible. On the other hand the respondent should be given as much information to help them understand the question but not enough to confuse them.

In order to do this the user interface must have the following properties:

- A question display. This just presents the question text to the respondent. This should be large enough and sufficiently clear for all people to read clearly.

- A response field. This provides facilities for the respondent to enter an answer. It may take the form of a listbox of predefined items or a edit field for entering text. It should be obvious how to enter the reply without addition help.

- A reply complete control. Once the respondent has entered their response they need to be able to indicate that they have finished entering their response. This can be done by clicking on a 'OK' or 'Continue' button on the screen with the mouse.

A user interface which implements these concepts should allow a respondent to progress through all the questions and sections in the questionnaire without trouble. 


\subsubsection{Implementations}

As indicated by the standard and extended versions of this model, there are two different implementations. Each implementation consisted of three programs: QuestED is the questionnaire editor, QuestEX is the questionnaire administration program and QuestView is the questionnaire summary viewing program. QuestEX is the key program in both implementations for a number of reasons:

- It is the program that administers the questionnaire to the respondents.

- It must be able to interpret the questionnaire structure.

- It must be able to clearly present the questions and receive a reply.

- It must be able to produce a summary of the respondent's replies.

These reasons are discussed further also with the implementation of QuestED and QuestView in the following sections.

\section{Standard Question Order Flow Implementation}

This implementation was the first Microsoft Windows 3.1 based implementation of QUEST. This had a number of advantages over the first LifeQuest program:

- Virtual memory. This overcame the $640 \mathrm{~Kb}$ DOS memory limitation. This limited the complexity of the LifeQuest program and the size of the LifeQuest questionnaire. The Windows virtual memory manager allowed the implementation of large programs with an extremely large memory heap.

- A standardised user interface. The Windows user interface provides a common appearance to a set of common functions. Consequently only a small set of user actions need to be learnt in order to be proficient at operating the Windows user interface.

- Graphics Device Interface (GDI). This provides a transparent interface to a large number of hardware display devices. This device independence allows for a powerful set of graphics primitives which can be easily manipulated on different display hardware.

However the first implementation required a different approach to that of console type programming. Windows programs are event driven, which does not suit a recursive depth-first search algorithm. An early version of QuestEX was implemented with such a recursive depth-first inference engine, however while this version worked well initially, it would crash spectacularly after processing around twenty questions. This happened 
because Window 3.1 is a cooperative multi-tasking system. The inference engine would not yield to the system, therefore it denied the operating system a chance to perform tasks such as updating the screen and freeing system resources.

This problem was solved by using a state machine approach. This approach used a Windows timer to act as an event generator. This timer is set up as a one shot timer, with a period of 0.25 seconds. It is started at the beginning of a questionnaire session. When the timer event fires a global variable containing the current block identifier (state) is retrieved. A search predicate is then called to determine the next block identifier to ask based on the current block identifier and the respondents replies. This is similar to finding the next state in a state machine from the current state and the state machine inputs. Once the next block identifier is known, the associated question can be asked. Once the question's reply has been retrieved and stored, the one shot time is activated again and the process is repeated. The use of the timer is important as it gives the operating system an idle period in which it can perform its system duties. Figure 3.7 contains a simple version of the timer event handler.

Figure 3.7 The standard depth-first search algorithm.

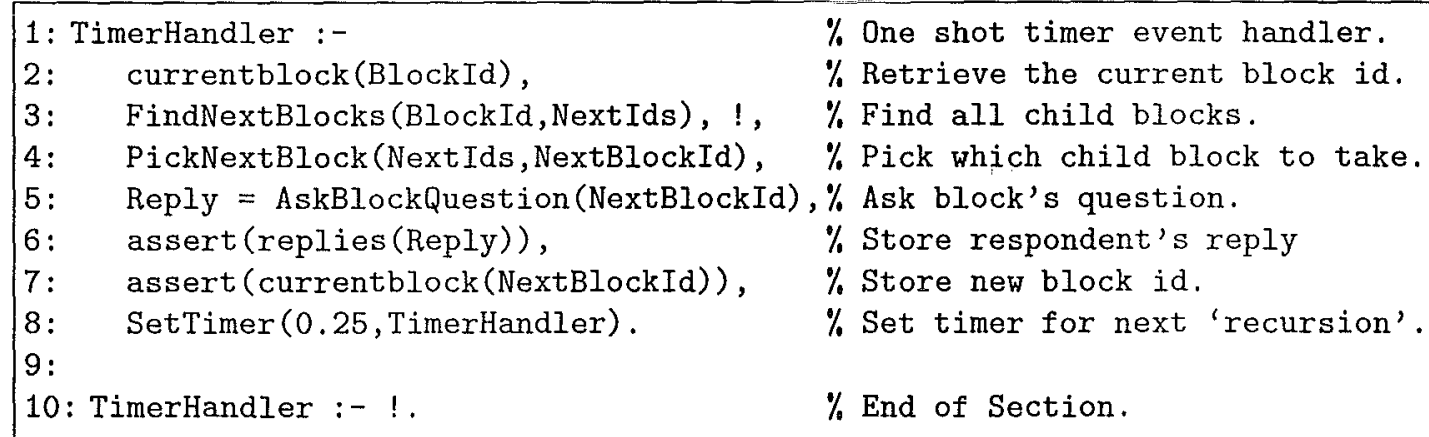

This algorithm is similar to the basic search algorithm as presented in figure 3.4. The main differences are:

1. Use of the timer to call the algorithm and each iteration, line 1 and 8 .

2. The current block identifier is saved in the global database currentblock instead of being passed as a argument in the basic version, lines 2 and 7 .

3. The basic version uses a back tracking approach to find the next child block. This next block's link conditions are evaluated and if false, the algorithm backtracks to find the next child block. This version finds a list of all the child blocks in line 3 using the FindNextBlocks predicate. The PickNextBlock predicate in line 4 then determines which block from the list should be followed.

The need to call predicates to perform the tasks in lines 3 and 4 is non-determinism. A Prolog program running under Windows is not allowed to include any non-deterministic predicates. Backtracking is one of the main causes of non-deterministic predicates 
in Prolog. Non-deterministic predicates can be removed by careful use of recursion techniques. The FindNextBlocks predicate can still fail, which signifies that there are no more child blocks to process. In this case the TimerHandler predicate backtracks to the second clause in line 10 which immediately succeeds.

The QuestEX user interface was designed around a series of dialog boxes. There was a dialog box for each type of block. Each dialog box had a number of features in common. These are:

- Question text field: Displays the question or section text.

- Response field. This is where the respondent can enter their reply. Depending on the block type it be a text edit field or a scrolling list box field.

- A series of buttons. These are:

1. 'OK' button. Pressing this indicates that respondent has finished entering their reply.

2. 'Quit' button. Indicates that the respondent wishes to quit the current questionnaire session.

3. 'Back' button. Pressing this button takes the respondent back to the last question asked. It is useful when the respondent realises they have made an error in their reply to a previous question.

4. 'Help' button. Displays a simple help message which is intended to provide additional comprehension information for the current question. Also a list of reasons why the current question is being asked is displayed. Each question in a section has a 'why' reason which takes into account the link conditions that question. For the path through the section that the respondent has travelled, each block's reason is added to a list and is then displayed to the respondent. This gives a simple explanation of how their responses lead to the current question.

- An optional picture field. An area in which a windows metafile (WMF) picture could be displayed.

Figure 3.8 shows the typical style of these dialog boxes. The main advantage of using these dialog boxes was their simplicity. They were easy to setup and the arrangement of the controls within each dialog box could be manipulated with a standard Windows dialog box editor.

The summary facilities of this version were very limited. At the end each questionnaire session, the respondent's replies were formatted into a list of the questions and the associated replies. This list is shown in figure 3.9 which shows the summary display. From here the respondent could get a print-out of this summary list. 


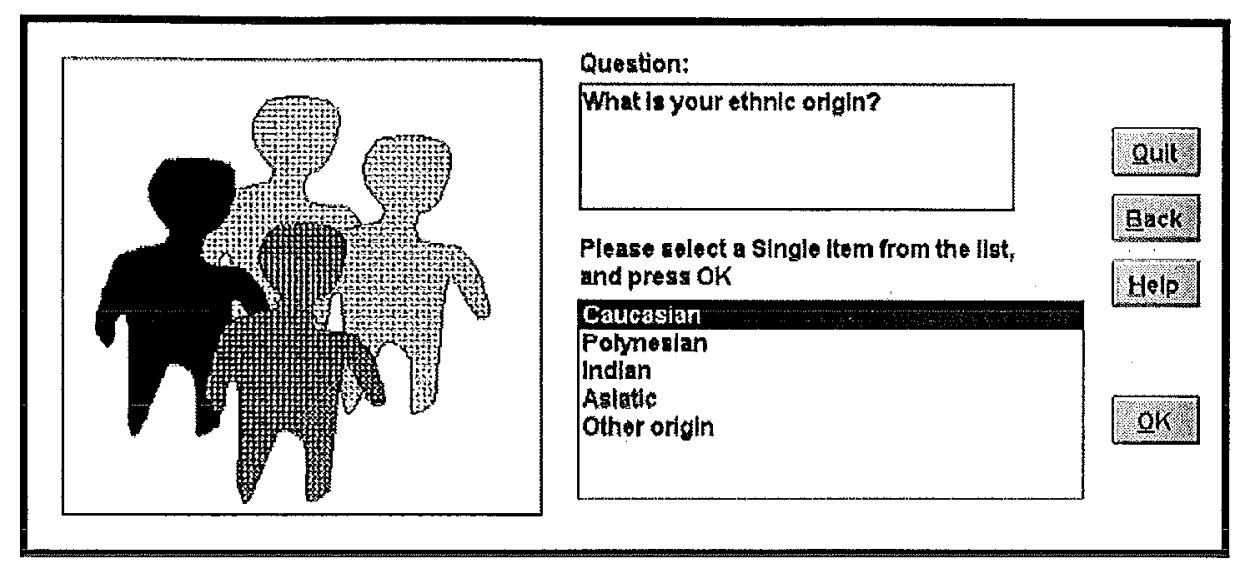

Figure 3.8 The dialog box for the single select list block type.

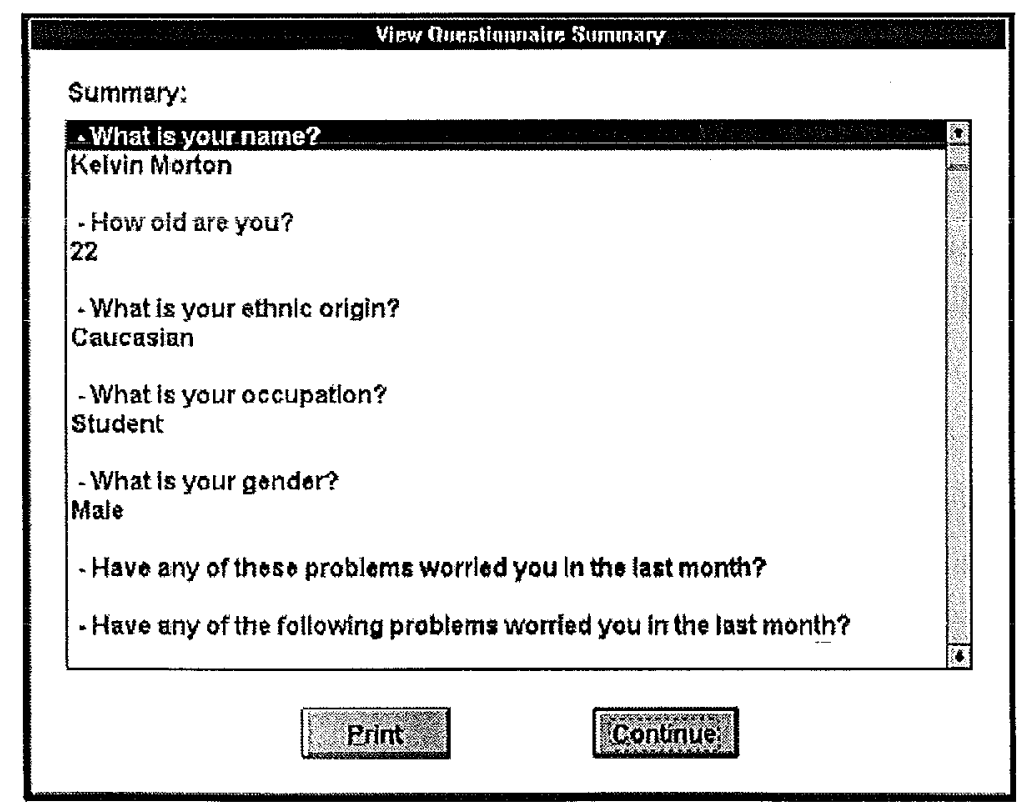

Figure 3.9 Dialog box of the summary display output. 
The replies are then saved into a simple database file. The QuestView program could then be used to load the reply files. Once loaded each file could be displayed and printed in the same manner as in QuestEX.

The questionnaire editor QuestED was initially based upon the original LifeQuest editor in terms of editing a questionnaire structure. It provided three main editing functions for the questionnaire header, sections and the questions.

Editing the questionnaire header allows the author to change the questionnaire title, questionnaire introduction, questionnaire background and exit password. Editing the sections allows the author to create and remove sections, and to change the section ordering and each section introduction and picture. Question editing is more complex. Firstly the author can create, delete and edit questions within a particular section. Once a question has been created, the question type, text, picture, help text and why text can be defined. Finally the question can be linked to other questions in the sections. This is done by selecting which questions precede the current question. The link conditions are created by firstly selecting a block id from a list of single select type question blocks. These single select type question blocks always precede the current block in the questionnaire. Secondly a reply is chosen from a list of predefincd responses for the selected block.

Initially these were the only editing functions QuestED provided. This provided little feedback to the author in terms of the questionnaire structure. It was difficult for the authors to clearly see any mistakes made during questionnaire entry. Consequently a tree graph facility was added to QuestED which showed to structure of the questions within a section. Figure 3.10 shows this facility.

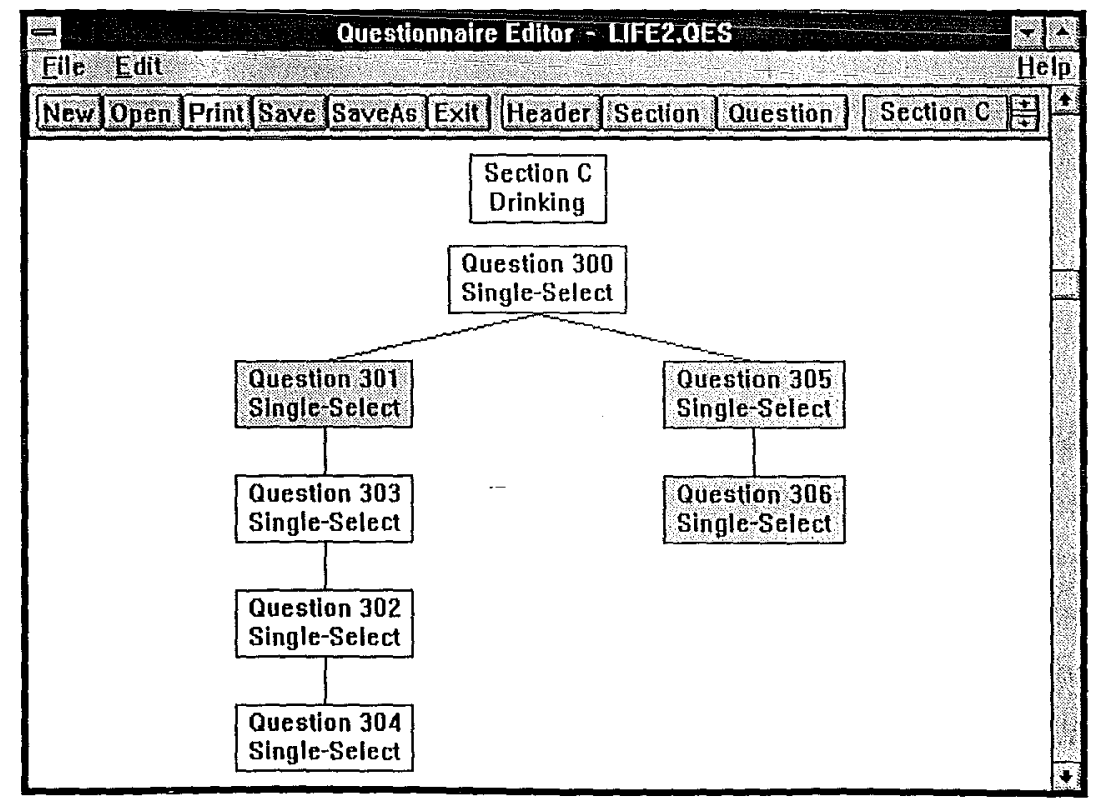

Figure 3.10 The QuestED tree graph representation of the standard question order flow model. 


\section{Extended Question Order Flow Implementation}

This implementation of the question order flow model was never completed. However many parts of it were carried over into the Required Information flow implementation. The most notable of the parts carried over is the template manager, which provides a customizable full screen questionnaire user interface without the restrictions of the earlier dialog box based approach. An early decision made for this implementation was to continue to use Prolog for the QuestEX program. The QuestED and QuestView programs would then use a mixture of Prolog for the questionnaire data management and $\mathrm{C}++$ for the user interface functions. Later this allowed the template manager to be used in the Required Information flow implementation almost without change as it written in Prolog. The QuestEX user interface and the template manager are discussed further in section 4.5 .

A prototype of the QuestED user interface was also made. The aim of this prototype was to test the interaction between the $\mathrm{C}++$ user interface modules and the Prolog data management modules. The Borland Object Windows Library (OWL) application framework was used to implement the QuestED user interface. The user interface was to based around a questionnaire view in which the flow chart style graph of the questionnaire appeared. Next to this graph was a panel which contained a series of block properties, hence the name property panels. Each block type has a different panel which resembles a dialog box fixed into the main window frame. Each property panel contains a number of fields which relate to specific properties for each block type. As the author selects a block from the flow chart graph, the details for that block are displayed within the associated property panel. The author can then modify any of these properties which are saved when another block is selected.

The prototype was able to establish that the property panel method for displaying the block information was satisfactory. The property panels could be displayed quickly containing all the necessary block data. All the relevant information concerning a block was then available to the author at a moment's glance. This made it easy to scan through a number of blocks in order to find a particular item. However the prototype was able to establish that the methods for displaying the flow chart block graphs lacked performance. Even though a fairly crude algorithm was being used to draw the chart, the drawing process slowed down significantly once more than 30 blocks were displayed on the screen. While this was not a large problem on a fast pentiumbased IBM compatible computer, on slow machines the delay would be unacceptable. It became apparent that a single program written in either Prolog or $\mathrm{C}++$ would perform better, because of the overhead in structuring a program coded in two very different programming languages.

The main problem with this implementation was with consistency checking. As previously mentioned the task of ensuring that the author has produced a consistent 
questionnaire is not trivial. The actual process of checking the questionnaire takes a reasonable amount of time. One method for performing this checking is path unwinding. As the questionnaire structure is a tree graph with well defined start and end points, all the possible paths through the questionnaire can be created. Each path would have a list of link conditions, and all the referenced blocks within the list must lie down that path. If a referenced block can not be found down a path, then that reference is inconsistent. For an increase in size of the questionnaire, the time to check the questionnaire grows disproportionately. The resulting delay would prove very disconcerting to the questionnaire author, especially when in general the author is likely to make relatively few mistakes when entering the questionnaire for the first time. More mistakes would be expected when the questionnaire was being revised.

The following cases show a number of the alternative consistency checking methods considered:

1. In the worst case, the consistency checker would process the whole questionnaire after every change to the questionnaire structure. This would introduce an unacceptable delay for the author after each change.

2. Only the current section would be checked as any error would likely occur locally to where the author was editing. Any references to blocks within other sections could be ignored. Although this would catch the majority of errors within a section, those errors which are ignored are probably more serious in terms of maintaining the questionnaire structure.

3. As for item 2 except that any references to blocks within other sections would be processed at a section level. After editing questions within a section, all the possible branches within that section would be stored along with all the link conditions for each branch. In this way any reference to that block from another section would only need to look up this list of branches for that section. If the reference is to a block which lies in a conditional branch, then consistency cannot be guaranteed as it depends on firstly the condition and secondly the respondent's reply.

4. One of the above methods could be available as a function that the author can invoke. In this way the author can choose when to check for possible errors. The author could also have a choice of the check complexity, for example a simple test would just check the current section as in method 3 or a full test would check the whole questionnaire as in method 1 . Full tests could also be performed after loading and before saving a questionnaire.

Each method would display a list of the warnings to the author. Each warning would have to be addressed before the author could proceed. This could be a matter of fixing the problem completely or just dismissing or ignoring the warnings. 
While all these consistency checking methods were investigated, it was decided that:

- The actual implementation of any of these schemes would slow the editing process down too much for the author. It would not be an intuitive process for the author to continually check for these errors. Especially if the questionnaire has been well designed in the first place. In this case there should be not structural errors, although errors may occur when entering the questionnaire data.

- It would difficult to $100 \%$ guarantee a error free questionnaire after the checking process. The checking process could only spot consistency errors which have actually been identified. If the model still contained flaws which had not been classified then the consistency checker would not spot them, and unpredictable behaviour could result.

- The use of such a tool is a reactive measure. We are trying to spot an error which has already occurred. It would be simpler in the long run if the questionnaire structure had some in-built characteristic which avoided the problem of consistency checking.

\subsubsection{Summary}

A number of problems with the Standard Question Order Flow model had been discovered in the course of its design and implementation process. These problems are:

1. An untraceable database bug in the editor. This reduced reliability and useability.

2. Only a simple print-out of respondent's answers was available as a summary. No form of 'intelligent' summary could be generated. This requires extra work for the author to extract the required information from the replies.

3. The only response type offered was the text string. This meant that the inference engine could not easily manipulate or process the responses.

4. The inference engine was unable to take advantage of a reply to a multiple select list question. Any replies to a question of this type could only be displayed in the summary. Therefore conditional branching was unavailable for question replies with multiple entries.

5. The inference engine had no ability to combine the replies from a number of questions. It could perform a conditional OR branch on the responses from a number of single select list questions. But it could not execute any sort of mathematical or relational expressions. 
6. The questionnaire editing interface was largely unchanged from the original version. A hierarchy of three dialogue boxes provided the means for editing the questionnaire header, the section headers and the questions.

7. The complete flow of a questionnaire section was not completely obvious even with the tree graphics. The tree graphics provided only a limited amount of information about the questions in a section. For each question only the type, id number, the links to and from, and the existence of a condition were apparent. To find complete information about a question, the author would have to traverse through a number of dialogue boxes.

8. The possibility for consistency errors existed. This was due to the fact that a question could contain references to another question within the structure of the questionnaire. A number of possible types of inconsistency could then occur. The referenced question could be deleted. The question containing the reference could lie before the referenced question. The referenced question could also lie down a branch in the flow which may or may not be traversed.

9. Although user interface for the questionnaire display program was adequate, it lacked flexibility. Every questionnaire had the same appearance. Each question form consisted of standard Windows buttons and list boxes. The questionnaire author was largely unable to customise the appearance of their questionnaire. A customised appearance would appear more attractive to the respondent, especially if it fitted the surroundings, e.g. using a medical centre's logo.

In response to these deficiencies in the standard model an extended questionnaire model was proposed. The implementation of this model fixed many of the implementation related problems in the standard model. In addition the following extensions were made to the actual questionnaire model:

1. The number of question types was increased. Included in the additional types were numeric entry questions, the response of which can be easily processed. The list type questions were expanded, so that a selected response returns three pieces of information; the response's text string, the response's list index and an author defined numeric value.

2. A conditional 'AND' type branch type was added to the inference engine functionality. This type of branching consists of a number of sub-branches. Each sub-branch is associated with a single responses from a multi select list reply. A return block terminates each end of the sub-branches. A default sub-branch provides the means for continuing past the conditional 'AND' branch. If none of the responses in the multi select list was chosen, then the default sub-branch 
is taken immediately. For every selected response the associated sub-branch is followed.

3. To enable the combination of a number of question replies, a calculation block is used. The calculation block is able to evaluate mathematical, relational and logical expressions. This block uses a response's numeric value or list index in a mathematical expression. The numerical value, list index and the text string can be used in a relational or logical expression.

4. The ability to generate multiple summaries was added. This was accomplished by defining a separate summary structure and mechanism. The questionnaire still operates in the same manner, but once finished the summary mechanism post-processes the respondent's replies. More than one summary can be defined for each questionnaire, e.g. a summary for the author, the respondent and one for importing into a spreadsheet or database.

The problem of questionnaire consistency, while probably only of secondary importance to the author, initially proved a major hurdle. While it is possible for an author to produce an error-free questionnaire without the help of consistency checking, it was felt that it was necessary to help the author to learn the limitations of the questionnaire model. In the event, consistency checking proved to be too computationally intensive, unduly hindering the author in attempts to create a questionnaire. What seemed necessary was a questionnaire model with a form of questionnaire consistency built in.

\subsection{REQUIRED INFORMATION FLOW APPROACH}

The Required Information model was designed to overcome the need to ensure consistency in the questionnaire structure. The main source of inconsistencies in a questionnaire stem from the use of references to other questions and the focus on the order of questions within the questionnaire. With the Question Flow model it was difficult to completely guarantee that a referenced question has actually been asked. The Required Information model takes a different approach by still grouping related questions together, but not rigidly defining an order for the questions. The focus is instead on the information flow. In this model the links between goals represent a child-parent relationship. This relationship states that the parent goal requires a piece of information from the child. Thus references to other goals can be guaranteed since as the link between the parent goal and the child goal also defines the question order. 


\subsubsection{The Questionnaire Structure Model}

The main components of the Required Information model are the goals themselves. These goals are similar to the blocks used in the Extended Question Flow model. Like a block their purpose or 'goal' is to gain or derive some information relevant to the questionnaire at hand. The information is stored as the same text list, index list, variable list structure as in the Extended Question Flow model. The main difference is that each reply structure also stores a goal state along with the response. There are a number of types of goals, namely:

1. Question goal. The question goals are basically the same as their Extended Question Flow equivalents. For all types of question goals, the reply state will be true, however this can be changed by a condition goal.

2. Condition goal. All types of goal may have an associated condition child goal. The condition goal provides the means for changing the state of its parent goal. The condition goal itself performs a relational expression. It is executed before its parent goal so that the result of the condition can determine the state of the parent. If the condition evaluates true, then the parent goal will then be executed, otherwise the parent's state is set false and it is skipped. The condition goal obtains the necessary information to evaluate the condition from its own child goals.

In the simplest case there would be one sub goal. An example is shown in Figure 3.11. When the parent goal G1 is executed without the condition goal, the two children Q4 and Q5 are executed in order. However with the condition goal C2, the condition goal is executed before the parent. Because the condition goal doesn't have enough information to complete its execution, it must execute its own child goal Q3. Upon executing goal Q3, the respondent is asked the question "Do you want to continue? $(\mathrm{y} / \mathrm{n})$ ". The reply to this question is stored as the reply to goal Q3. Once its child goal has finished executing, the condition goal now has enough information to evaluate "Q3 = ' $\mathrm{y}$ '", which it does. The result of this condition is either true or false depending on the respondent's selection. Now the condition goal has finished, the parent goal G1 examines the result of the condition goal. If it is false, then the parent goal aborts its own execution and sets its own state to false. However if the result is true, the parent's state is set to true and the two children goals Q4 and Q5 are executed. This provides a means for creating skipping patterns within the questionnaire.

3. Group goal. The group goal provides a mechanism for controlling the questionnaire flow over a number of goals. It in effect takes a number of goals and 'groups' them together so they appear as one. This allows the author to enforce a hierarchical structure on the questionnaire as a group goal may appear as a sub goal 


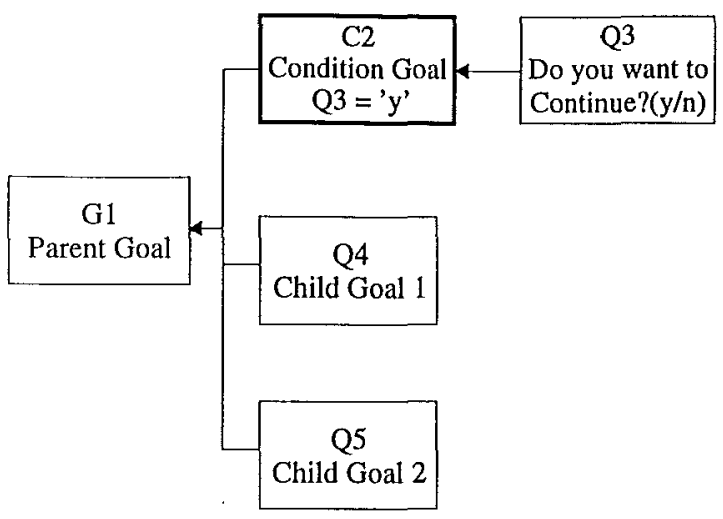

Figure 3.11 This example shows the operation of a condition goal.

of a parent group goal. An example of this is when number of related questions are grouped together into a section of questions. This is the same concept as the sections in the Question Flow model. However the group goal is more powerful as it may further subdivide a section of questions into sub-sections. If a group goal has a condition sub goal, then this condition determines whether the group goal's other children as processed. The information collected by a group goal flows no further up the questionnaire tree structure. It may however be referenced by other goals or a summary. Figure 3.11 demonstrates this situation where the parent goal G1 is a group goal.

4. Calculation goal. The calculation goal is similar to the Question Flow calculation. Its purpose is to combine the replies from any number of responses. The actual calculation is composed of any number of sub goal references and mathematical operators, Relational, logical and arithmetical calculations are possible. The referenced sub goals are directly linked to the calculation goal as in the Group goal case. If during the execution of the sub goals, the state of a sub goal is false then any remaining sub goals are ignored. This is because the calculation goal can no longer obtain all the information it requires to evaluate the calculation. The calculation goal's state is set to false as a result to indicate that the calculation failed.

5. Info goal. The info (information) goal is complementary to the question goals. Whereas the question goals gather information from the respondents, the info goal provides information feedback to the respondents. It is similar in structure to the group goal as it can group sub goals together, but the sub goals are executed first. The information the sub goals collect can be presented to the respondent in a fashion chosen by the author in the info goal. Thus the author can provide feedback on the output of any goal in the questionnaire. For example see Figure 3.12 , 


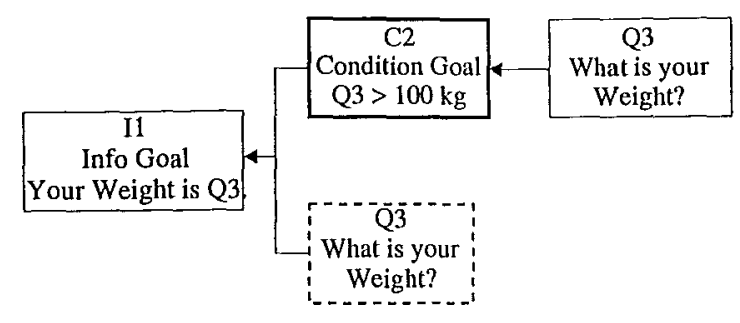

Figure 3.12 This example shows the operation of the Info goal type.

When the info goal I1 is executed it first executes its condition goal C2. The Condition goal in turn executes the question goal Q3. This goal obtains the weight of the respondent. This condition goal determines whether the respondents weight is over $100 \mathrm{~kg}$. If it is not, then the condition is false and the Info goal does not execute it's other sub goal or display its information to the respondent. If the condition is true then it will execute its other sub goal. Note however that this sub goal is question goal 3 again but this time it is being referenced. This is indicated by the dashed border of the goal. Thus the info goal can take the result of question 3 and format it in the information text, "Your weight is Q3". This is then displayed to the respondent.

Simplistically the structure of a questionnaire can be created by joining a number of question goals to parent group goals. These group goals form the questionnaire sections. They then join to a single parent group goal which forms the questionnaire introduction goal.

\section{Questionnaire Model Interpretation}

The model is interpreted by using a recursive depth-first search routine. Because a questionnaire structure forms a hierarchical tree, the search engine can be started from any node within the tree and will call itself during the processing of the tree branches. An important difference from the depth-first technique introduced in section 2.2.4 is that there is no one particular solution goal in the questionnaire. So the depth-first search routine will attempt to process every goal in the questionnaire space. Therefore there is no need to find a solution path as this may include every goal in the questionnaire space. The depth-first search routine is based on two lists, called Open and Closed. The Open list contains the goals that have been generated but have not been processed. The Closed list contains all those goals that have been processed. The pseudo-code in figure 3.13 demonstrates the basic approach (Luger and Stubblefield, 1989, Ch. 3),

Line 7 is important as it is when the functionally of each goal is actioned. This functionality depends entirely on the type of the goal. The details of this and the recursive implementation of the above algorithm are discussed further in 4.3. 
Figure 3.13 The pseudo-code approach for the depth-first search algorithm.

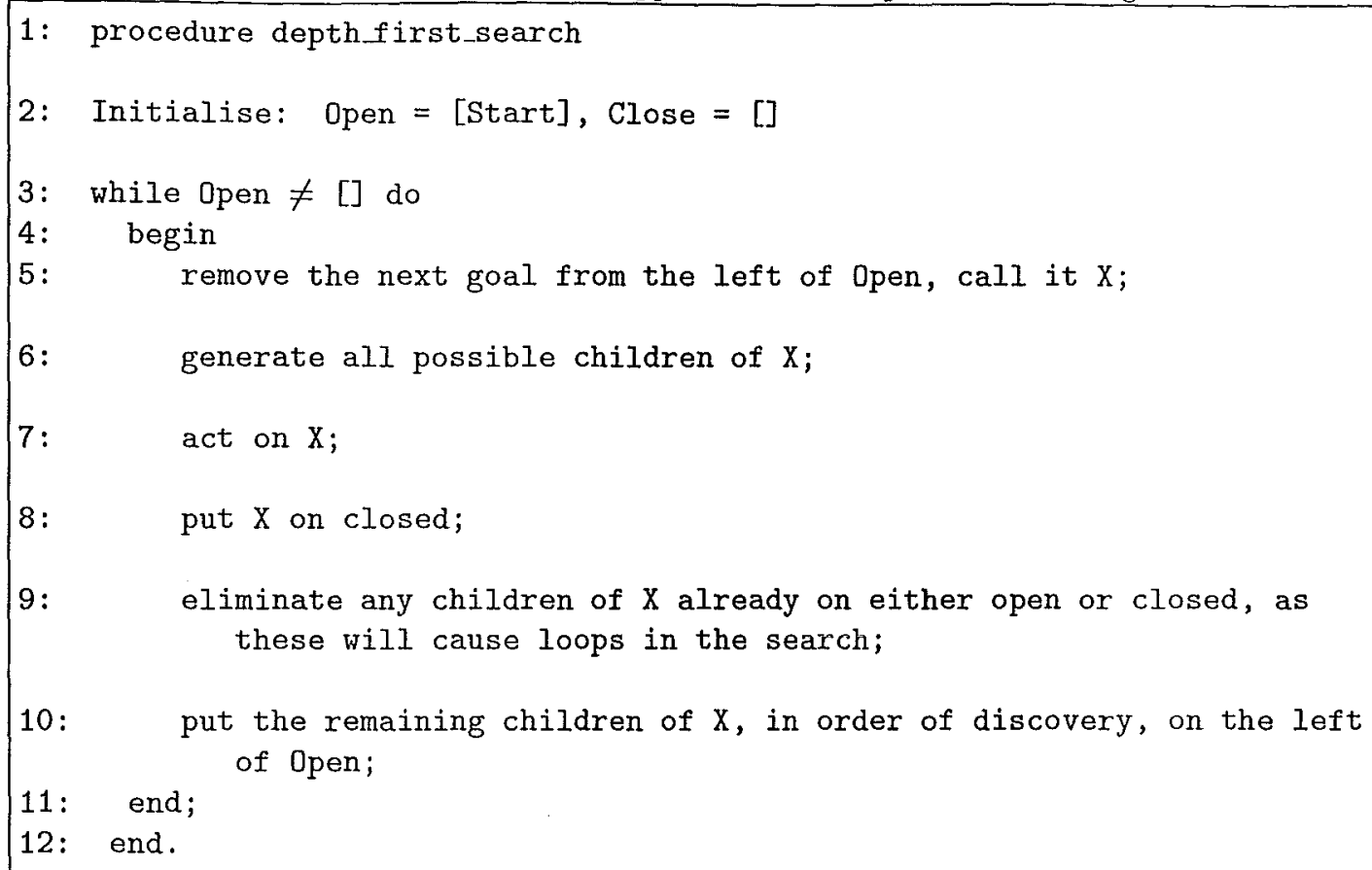

\subsubsection{Implications for the Author}

The Required Information questionnaire model is initially more difficult to master than the Question Order flow model. This is because the aim of the model is much deeper. The Question Order flow model focuses on the somewhat superficial aspects of questionnaire design, namely the ordering of the questions. This is an easy concept to understand as most people have been questionnaire respondents at some time. The respondent's main impression of the questionnaire is a sequence of related questions. However the author is more concerned with the information the questions provide rather than their order. The need for enough quality information from a questionnaire to prove or disprove a hypothesis is the most important factor for the questionnaire author.

The Required Information flow model is based around this idea of gathering information. The author must then define what information is required for the survey hypothesis. This information must be broken down into progressively smaller subhypotheses, until it is possible to ask a single question for each sub-hypothesis. This process should ideally be carried out regardless of how the questionnaire is to be implemented. However the Required Information flow model requires this type of approach from the very beginning of the questionnaire process.

The Question Order flow model allows the author a more relaxed approach when defining the questionnaire information hierarchy. The author can take more liberties with the structure of the questionnaire, and so be more vulnerable to irregularities 
in the sequence of questions. These irregularities show up as inconsistencies in the questionnaire structure.

The Required Information flow model maps the questionnaire information hierarchy onto the questionnaire structure. This guarantees the consistency of the questionnaire as each branch in the questionnaire structure deals with the same information, except at different levels of detail. The author can still modify the order of individual questions in the questionnaire, but only within the same level of branch. So while the author has to deal with a more conceptually challenging questionnaire model, they gain the benefits of a structured approach to questionnaire design and guaranteed questionnaire consistency.

\subsubsection{Implications for the Respondent}

The implications for the respondent should be no different with this model from the Question Order flow model. The respondent still will see the sequence of questions in the same order for the same questionnaire.

\subsubsection{Implementation}

The implementation of the Required Information approach is outlined in the following two chapters. Chapter 4 describes the implementation of the Required Information model for the questionnaire administration program, QuestEX. It describes the implementation of the inference engine, the user interface and the summary generation facilities. Chapter 5 discusses the implementation of the questionnaire editing tool, QuestED. It overviews the questionnaire editor, the user interface editor, and the summary editor. 



\section{Chapter 4}

\section{QUESTEX IMPLEMENTATION}

QuestEX is the name of the program used to implement the Required Information questionnaire model discussed in section 3.4. The QuestEX program was implemented using PDC Prolog for Windows. PDC Prolog includes all the standard bindings to the Windows Application Programming interface (API). A large proportion of the Windows API functions are available as defined in the Microsoft Win16 API Programmer's Reference. A small proportion of these functions can not be correctly handled by Prolog. These functions typically involve array handling which is not a predefined type in Prolog. Therefore a number of helper functions are available via the PDC Prolog Windows bindings to allow access to these functions.

QuestEX consists of a number of components. These components and their function are described in the following list. Figure 4.1 shows how the components interact with each other:

- MEX. The main QuestEX module. Handles initialisation of the program as a Windows Application.

- ENG. The QuestEX Inference engine. This module controls the execution of the questionnaire.

- CALC. The Calculation module evaluates any calculation and condition goal expressions.

- LOG. The File Log module provides a simple log file service.

- INFO. The Information goal module generates the text used by the information goals.

- SUM. The Summary module generates the questionnaire summary after a questionnaire session.

- TMP. The Template manager is responsible for creating, displaying and overseeing the user interface aspects of QuestEX. 
- DBX. The Database manager handles the memory storage needs of QuestEX. This include loading the external database questionnaire files, storing the replies internally and saving the raw replies in an external database.

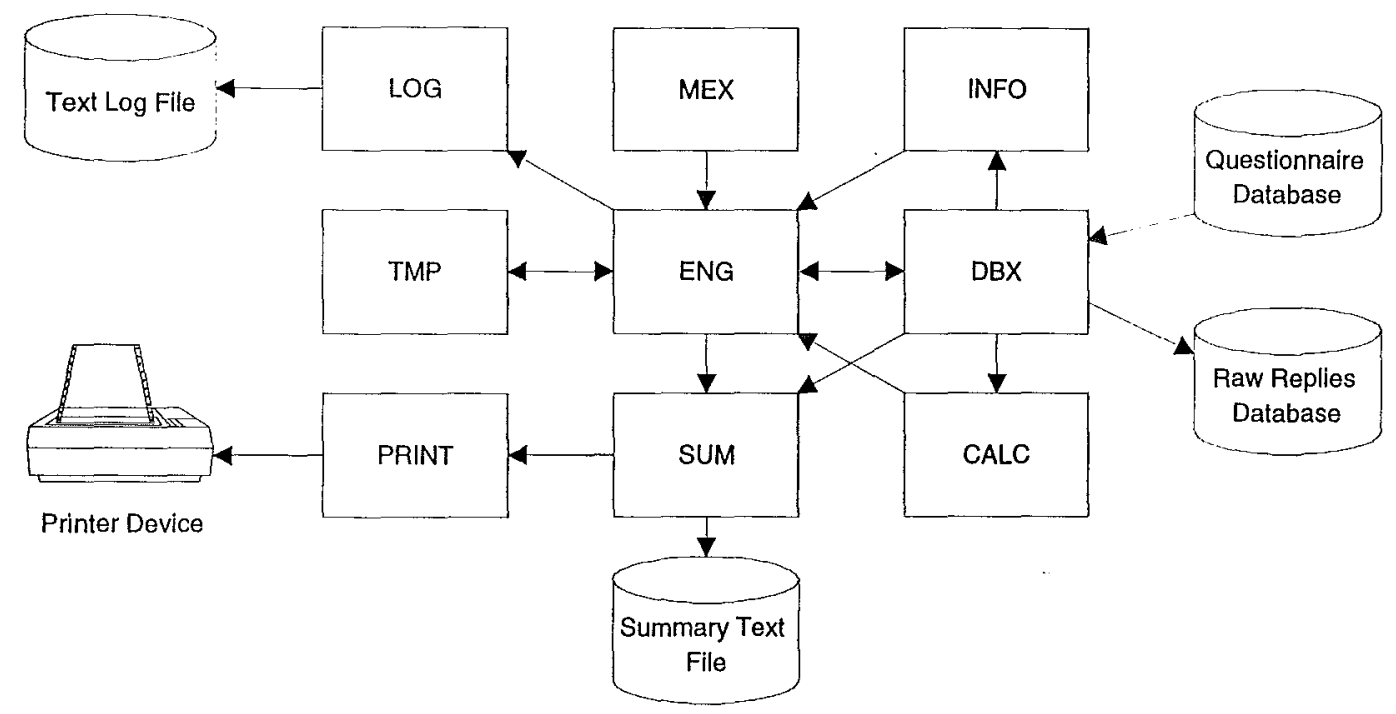

Figure 4.1 The structure of QuestEX's modules

The ENG inference engine module is the most important module in the program. It controls how the questionnaire is presented to each respondent. Dividing the program into the modules listed above allowed the development of each module to proceed as independently as possible. This required that the interfaces between the modules be defined at an early stage.

This modularity proved important when the decision was made to change the inference engine from the Question Flow model to the Required Information model. It allowed the specifications of QuestEX's other major components to remain unchanged. For example the User interface component is identical for both implementations while the Summary component requires only minimal changes.

The following sections discuss each of the components presented in Figure 4.1.

\subsection{INITIALISATION}

The initialisation of QuestEX occurs in two stages. The first stage occurs when QuestEX begins as a Windows program, and the second stage when the QuestEX main window is created. The steps involved in the first initialisation stage are:

1. Initialise the printer module.

2. Load the questionnaire file.

3. Create the main QuestEX Window. 
4. Set up any security options.

The QuestEX printer module must be initialised before the questionnaire is loaded. This is because the printer selection and setup options are available from the load questionnaire dialog box. So it is necessary to save the default printer state before the questionnaire administrator chooses which questionnaire file to load.

The filename of the questionnaire can be presented to QuestEX in two different ways. The first method is via the program line parameter. This can be accomplished by either specifying the questionnaire filename after 'Questex.exe' in the Program Manager icon properties or by double clicking on a questionnaire file with the correct file association setup. An attempt is made to load the questionnaire filename specified on the command line into QuestEX.

The second method displays a modified GetOpenFileName common dialog box as shown in figure 4.2. This dialog box allows the user to select a questionnaire file by manipulating the file and directory list boxes. An 'Exit QuestEX' button provides a method for quitting QuestEX at this stage. After selecting a questionnaire file, the 'OK' button starts the questionnaire. The 'Printer Setup' button displays the Print Setup common dialog box. From this dialog box the user can select the desired output printer, and the printer configuration can be changed via the 'Options' button in this dialog box. Once the filename has been obtained, QuestEX attempts to open it. If this questionnaire load fails because the file does not exist or the file is the wrong format this method is repeated until a suitable file is found or the user presses the 'Exit QuestEX' button.

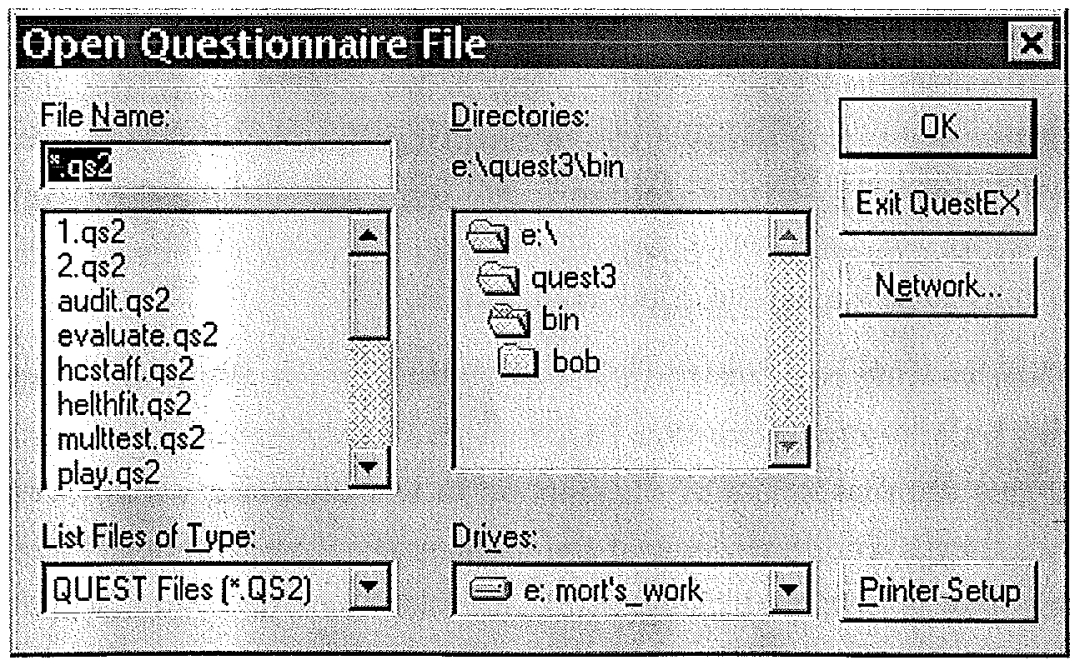

Figure 4.2 The QuestEX open questionnaire file dialog box.

Once a questionnaire has been loaded, the main QuestEX window is created. This is done by registering an application window class. The most important information the application window class contains is a pointer to the main window procedure. From 
the window class a maximised popup window is created. This produces a full screen window without any decorations such as title bars, borders and system buttons. This window serves as the main application window. The purpose of the main application window is just to manage the remaining initialisation steps and to be a parent window for the template window.

If the 'Safe Mode' questionnaire option has been enabled, task switching must be disabled while QuestEX is running. To achieve this the main application window is made 'System Modal'. When a window is system modal then only that window can receive input messages from the keyboard or the mouse. All other programs running in the system effectively are stopped from running. An example of a system modal window is the 'Exit Window' dialog box which appears when closing Program Manager in Windows 3.1. Optionally a virtual keyboard driver can be installed when Windows starts. This keyboard driver operates at a system level and it used to intercept the 'Ctrl-Alt-Del' key combination. This driver is initialised when QuestEX starts and disabled when QuestEX closes. Thus when in 'Safe Mode' QuestEX effectively takes over the operating system until QuestEX is closed.

Once the main application has been created a window initialisation message is sent to the main window procedure. The steps involved in the second initialisation stage are:

1. Initialise the Inference Engine.

2. Initialise the Template Module.

3. Initialise the Log-file module.

Initialising the inference engine involves resetting the two global databases used by the inference engine. The first database is the responses database which holds the replies entered by the questionnaire respondent. The second database is used while processing the reverse polish notation calculations.

The template module requires the most initialisation. A Windows class must be created along with the template window based on this class. Again the most important information in this template class is the pointer to the template windows procedure. The template window is a child window of the main application window. The resolution of the screen display is obtained using the GetSystemMetrics system call. The size of the template window is then set to this resolution is ensure a full screen display. The four questionnaire buttons windows are then created as children of the template window. These buttons are only created during initialisation as they can be easily enabled and disabled and moved around once the questionnaire is running. The buttons are also subclassed. This means a message hook is installed into each button's own window procedure. Any messages that are sent to the buttons can be caught and 
processed. This is discussed further in section 4.5. Finally the questionnaires FileInfo object is stored globally in the template module. The FileInfo object contains the questionnaire options which apply directly to a number of template functions. For example if the questionnaire has a password, this must be prompted for when exiting the questionnaire from the questionnaire introduction screen.

The Log-file module is initialised by checking for the 'Enable Log File' option in the questionnaire options. If the option is enabled, then a log file is created in the same directory as the questionnaire file.

\subsection{DATABASE MANAGEMENT}

QuestEX has three main external data stores. These are: the questionnaire file, the raw replies file and the summary file. In addition the temporary response database forms an internal data store. Figure 4.3 is a data-flow diagram which shows these data stores and the data flows between them.

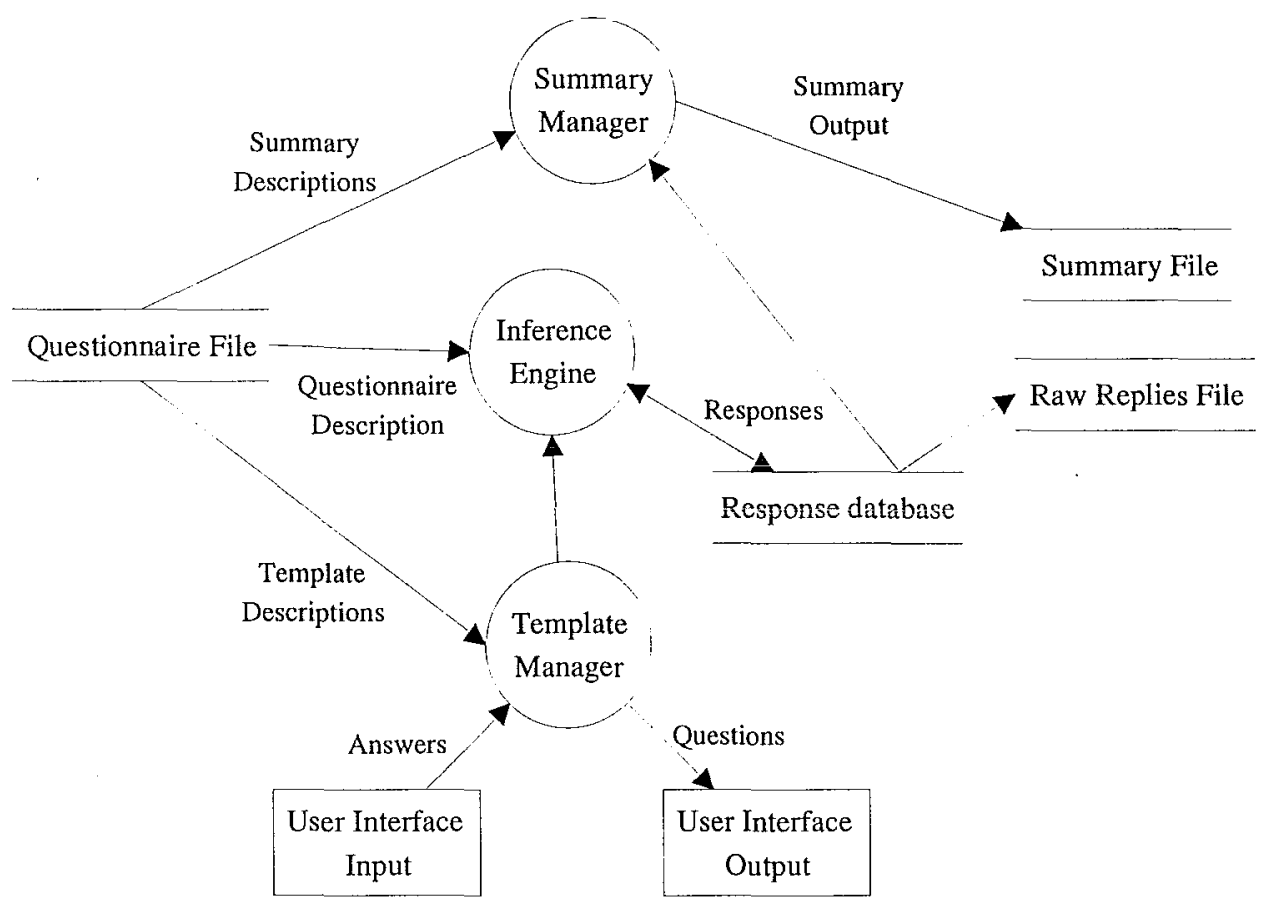

Figure 4.3 Top level Data Flow Diagram for QuestEX.

The Questionnaire file is a Prolog external database which contains three main sources of information: the questionnaire description, the template descriptions and the summary descriptions. A Prolog external database is a binary representation of Prolog domain objects. Objects of the same type are stored in chains and there can be as many chains as required. There are separate chains for the questionnaire description, goal objects, question objects, template objects and summary objects. Each chain is 
a serial list of objects, but this list can be indexed by a binary tree created in parallel with each chain. The binary tree is searched using an ASCII search string as a key. The goal, question, template and summary are all indexed by a numeric identifier which is converted into a two character text string. This key string is used as the binary tree search index.

The domain declarations for the goal and question objects are:

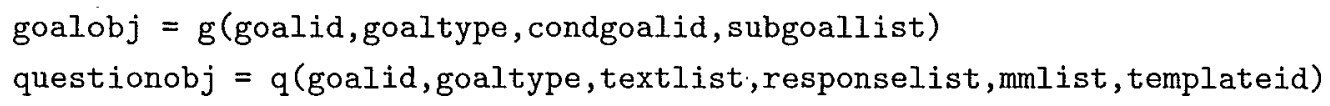

Each goal in a questionnaire has both goalobj and questionobj entries. The goalid and goaltype fields are repeated in both objects; this provides all the necessary information about a goal in different parts of the system. For example, goalobj is mainly used in QuestEX's inference engine and in QuestED's tree scanning routines. questionobj is mainly used in QuestEX's template manager and in QuestED question panel editor. The fields in these objects are:

- goalid - a unique integer identifier used to identify individual goals.

- goaltype - indicates the type of each goal. See table 4.1 for a list of goal type indentifiers.

- condgoalid - the goalid of the goal object's condition goal.

- subgoalist - a list of goalid identifiers for the goal object's sub goals.

- textlist - a list containing textlist items. These items typically contain textual question information or goal options

- responselist - a list containing response items. These are typically the predefined list responses for list type questions or reverse polish notation expression for calculation goals.

- mmlist - a list containing multimedia items. These define the multimedia events for the goal.

- templateid - an integer identifier of the goal's template object.

The template object and summary object domain declarations are:

templateobj $=t$ (templateid, NameStr, goaltype, ModeInt, ErrorStr, objectlist) summaryobj = s (summaryid, string, integer, sumprint, sumfile, sumentrylist)

Both of theses objects are discussed further in sections 4.5.1 and 4.6.1 respectively. The final object stored in the questionnaire file database is the file information object, it is declared as: 
Table 4.1 Goal type indentifiers (cf. goaltype)

\begin{tabular}{|c|l|c|l|}
\hline Goaltype & Description & Goaltype & Description \\
\hline gt_notype & Goal has no type & gt_group & Group goal \\
gt_calc & Calculation goal & gt_condcalc & Condition goal \\
gt_textentry & Text Entry goal & gt_numentry & Numeric Entry goal \\
gt_singlesimple & Single Select goal & gt_multisimple & Multiple Select goal \\
gt_info & Information goal & & \\
\hline
\end{tabular}

fileobj $=i$ (goallist, templatelist, summarylist, textlist)

This object is stores information about all the other object types in the questionnaire. The goallist, templatelist and summarylist each contain the a list of the all the object identifiers in the associated object chains. By recursing through each list, the object identifiers can be converted into the ASCII binary tree search index. This index references directly into the object chain obtaining the object in the most efficient manner. The final textlist list is used as a container for all the questionnaire options. Each option is stored as a option(optionid, value) field, where the optionid indicates the particular option. See appendix A for the full textlist declaration. Table 4.2 shows the possible values the optionid field.

Table 4.2 Questionnaire options identifiers.

\begin{tabular}{|c|l|}
\hline Goaltype & Description \\
\hline qopt_passwordenabled & Indicates if the questionnaire has an exit password. \\
qopt_passwordtext & The password text string. \\
qopt_logfile & Indicates if a log file is to be generated. \\
qopt_security & Indicates the level of the questionnaire security. \\
\hline
\end{tabular}

The external questionnaire database file is opened and copied in its entirety into memory. Once in memory it behaves in exactly the same manner as if it was still a disk file. However the advantage here is speed and as the size of a typical questionnaire is less than 100 kilobytes there is no problem with memory consumption. The memory database is used in a read-only fashion as presenting the questionnaire makes no changes to the questionnaire structure. When the questionnaire objects are required by various parts of QuestEX a series of interface functions retrieve the information. These functions are:

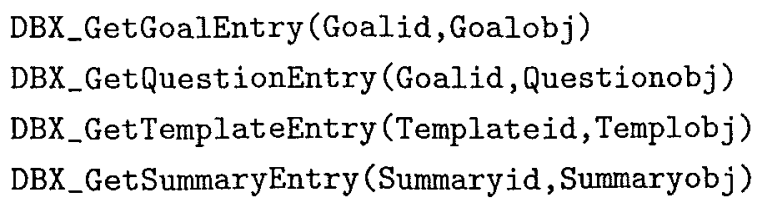

The identifier for the required object is converted into the ASCII binary tree search index. This index references directly into the object chain and obtains the object in the most efficient manner. 


\subsection{INFERENCE ENGINE}

The questionnaire inference engine is implemented in Prolog using a pair of recursive predicates. The reasons for this are:

1. A hierarchy tree is a recursive structure. For instance, each node in the tree can be treated as a parent to a number of child nodes.

2. The Prolog list type is best processed recursively. The Open and Closed lists introduced in section 3.4.1 are best implemented using Prolog lists.

Figure 4.4 shows the basic predicate implementation for such a recursive approach. For clarity unnecessary code has been removed.

Figure 4.4 The basic implementation of the questionnaire inference engine.

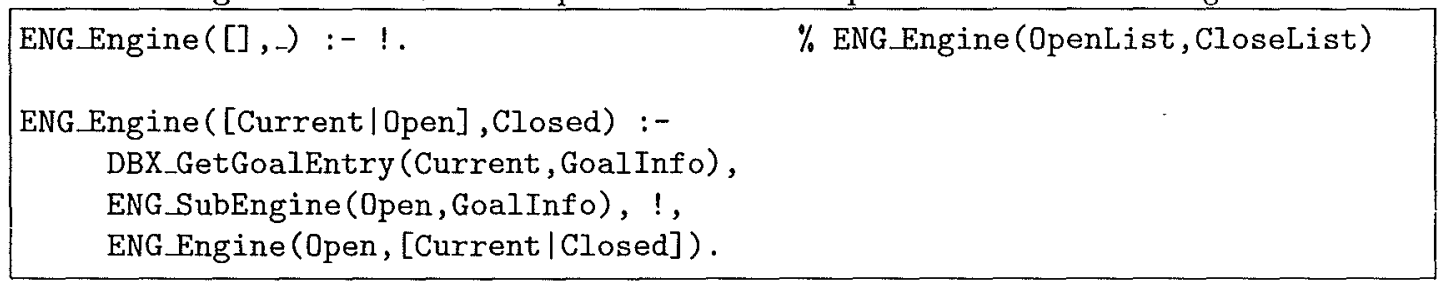

The first predicate is the recursion catch clause. This predicate succeeds when the Open list is empty and there are no more goals to process. As ENG_Engine is tail recursive the succeeding catch clause causes each clause in the recursion to succeed and so the predicate succeeds.

This is a very simple recursive predicate, the power and flexibility of the inference engine comes from a number of extra clauses and the ENG_SubEngine predicate. Currently the Closed list is not used. Figure 4.5 shows an slightly extended version which guards against processing the same goal twice. If a reply for Current goal already exists, then there is no need to ask the question for that goal again. This in effect performs a reference to the first instance of the Current goal in the questionnaire. The parent goal of the second instance of the Current goal can make direct use of this goal's reply as it already exists.

\subsubsection{The ENG_SubEngine Predicate}

The ENG_SubEngine predicate is essentially a large case structure with an entry for each different goaltype. In general for each goal type there are four sub-cases. These four cases arise from the ConditionGoal and SubGoal List fields of the Goalinfo variable pass to the ENG_SubEngine predicate. Each case relies on whether the Goal in question has a Condition goal or not, and whether the goal has sub goals or not. For each of the four cases, each goal type is generally handled in the same manner. A general description of each of the four cases follow. 
Figure 4.5 The method the inference engine uses to deal with goal references

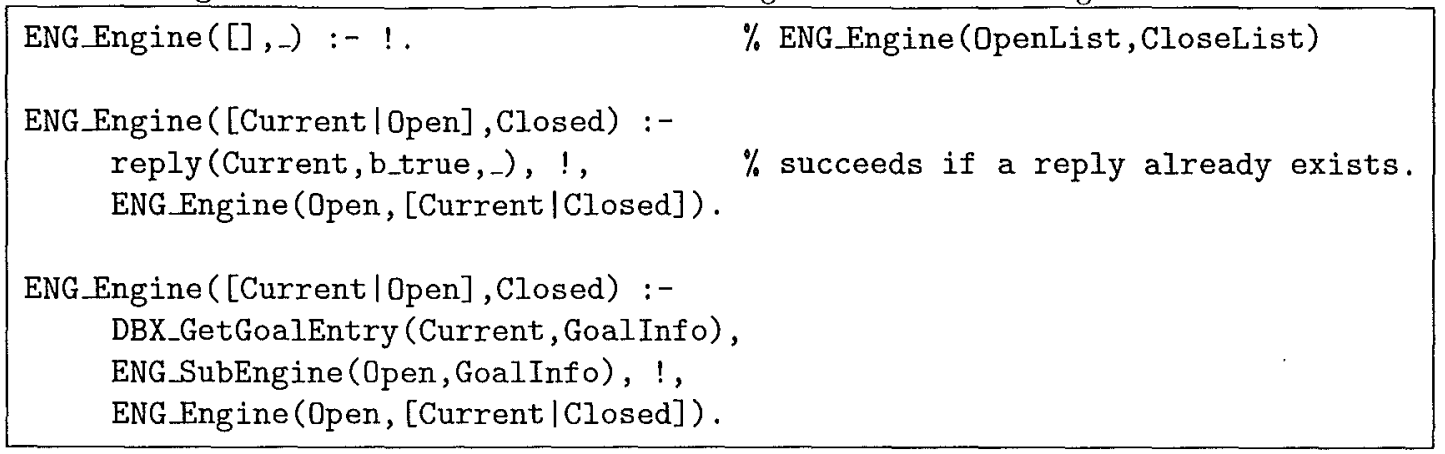

\section{Case 1 - No Condition, No Children}

This case occurs for non conditional question goals, as question goals have no child goals. This case has little meaning for condition and calculation goals as they require child goals to provide information for evaluating their expression. Info and Group goals can use this case, but a Group goal without child effectively performs the exact same function of a Info goal. The procedure for this case is generally:

1. Get the goal's question object.

2. Get the goal's template object.

3. Queue any sequenced music (see case 2).

4. Prepare and display the template.

5. Kill any queued sequenced music.

6. Check how the template was terminated.

7. Save the appropriate response in the reply database.

\section{Case 2 - No Condition, Has Children}

This case occursonly for control goals, not question goals,. There is no general case which for this case. Each control goal type has their own implementation. The Calculation, Condition and Info goals follow the following pattern:

1. Call the ENG_Engine predicate for the SubGoallist. This recursively processes all the children of the current goal. The children's replies are then be available for the parent goal to use.

2. Get the goal's question object. 
3. Execute the functionality specific to that goal type. For instance the info goal combines its information text with any goal references it makes. The Calculation and Condition goals evaluates their expressions.

4. Get the goal's template object.

5. Queue any sequenced music.

6. Prepare and display the template.

7. Kill any queued sequenced music.

8. Check how the template was terminated.

9. Save the appropriate response in the reply database.

It is important to note that as Calculation and Condition goals do not display a user interface they do not do steps 4 to 8 . The Group is similar but different enough to merit its own sequence of tasks, these are:

1. Get the goal's question object.

2. Get the goal's template object.

3. Queue any sequenced music.

4. Prepare and display the template.

5. Call ENGEngine predicate for the SubGoalList. This recursively processes all the children of the current goal. The children's replies are then be available for the parent goal to use.

6. Kill any queued sequenced music.

7. Check how the template was terminated.

8. Save the appropriate response in the reply database.

The Group goal does not have any 'functionality' in the sense of the other three controls goals. The Info goal is the closest to the Group goal in functionality except in the order of the tasks. The Info goal processes its children and then displays its user interface. The Group on the other hand displays its user interface first and then processes its children. Another important different of the group goal is with the sequenced music. The Group goal's music starts playing when it displays its template. The sequenced music is not be killed until after the children goals have been processed. This allows the group goal to manage a sequence of music for a whole section of child goals. This is why the sequenced music is handled in the ENG_SubEngine predicate when all 
the other multimedia functions are handled when rendering the template. More control is required over when the music is started and stopped, as music has typically a longer duration than a sound bite or an animation. See section Figure 4.5.4 for more discussion.

\section{Case 3 - Has Condition, No Children}

This case is similar to the first case except for the addition of the condition. The condition is processed in the same manner as the child goals, by calling the ENG_Engine predicate. When this predicate returns the result of the condition goal is available to test. The procedure for this case is:

1. Call the ENGEngine predicate for the Condition goal. This recursively processes the condition goal and any child goals it may have.

2. Test the result of the condition goal. If it is false then a 'false' response for this goal is saved in the reply database, and the procedure ends. Otherwise the procedure contiues.

3. Get the goal's question object.

4. Get the goal's template object.

5. Queue any sequenced music (see case 2).

6. Prepare and display the template.

7. Kill any queued sequenced music.

8. Check how the template was terminated.

9. Save the appropriate 'true' response in the reply database.

\section{Case 4 - Has Condition, has Children}

Again this case is very similar to case 2, the only difference being the condition goal processing. The procedure is only shown for the group goal:

1. Call the ENG Engine predicate for the Condition goal. This recursively processes the condition goal and any child of it.

2. Test the result of the condition goal. If it is false then a 'false' response for this goal is saved in the reply database, and the procedure ends. Otherwise the procedure contiues.

3. Get the goal's question object. 
4. Get the goal's template object.

5. Queue any sequenced music.

6. Prepare and display the template.

7. Calls ENG_Engine predicate for the SubGoalList. This recursively processes all the children of the current goal. The children's replies are then be available for the parent goal to use.

8. Kill any queued sequenced music.

9. Check how the template was terminated.

10. Save the appropriate response in the reply database.

\subsubsection{Back and Quit Functions}

The Back and Quit functions have been provided to allow the questionnaire respondent to have some control their questionnaire session. The Back function steps the questionnaire back one question, thus allowing the respondent to re-enter a question. The Quit function allows the respondent to exit the current questionnaire session. This returns QuestEX back to the questionnaire introduction.

Both the Quit and Back functions are closely related in their operation. There are three different states that the XUI_AskQuestion function can return after presenting the question to the respondent. These states depend on which button was pressed by the respondent. The states are:

1. Return State $=$ qs_ok. This is returned if the respondent pressed the 'OK' button. It indicates that the inference engine should proceed as normal. qs_ok is defined as the integer 0 .

2. Return State > qs_ok. This is returned if the respondent pressed the 'Back' button. The actual value of Return State indicates which goal was being processed at the time.

3. Return State < qs_ok. This is returned if the respondent pressed the 'Quit' button. The absolute value of Return State indicates which goal was being processed at the time.

This Return State is passed back from ENG_SubEngine to ENG_Engine where is also checked for. If the Return State is qs_ok then the questionnaire proceeds as normal. Otherwise ENG_Engine immediately returns this state to its calling predicate. As the whole inference engine is recursive this steps back out through the recursion until the original ENG_Engine predicate call returns. 
If the questionnaire has ended in the qs_ok state, then the respondent has completed the questionnaire normally. The summary can be generated and the replies saved. If the questionnaire ended in the Quit state, then two checks are made:

- If the Return State value is -1, then the respondent has pressed the Quit button in the Introduction goal. This is signal to shut down QuestEX. This is the only case that ends the Questionnaire execution loop.

- Any other Return State value indicates the respondent pressed the Quit button further into the questionnaire. This means the summary and replies save steps should be skipped. The previous replies are discarded and the questionnaire is then restarted from the beginning.

If the questionnaire ended in the Back state, then the current questionnaire has not actually ended. Ideally it would be possible to stop the inference engine, move it back one question and let it continue from there. However the inference engine only maintains an Open list of goals still to process at the current level of recursion. What would be necessary would be to have a complete record of previously processed goals carried around at each recursion level. However the problem is that each level of recursion into the questionnaire is a separate instance of the ENG Engine predicate. So it would be possible to step back through the goals in one recursion level. But to step back up a level would required some extra communication in the form of a status code. This increases the complexity of the inference engine in favour of a function which is typically the exception rather than the rule in the inference engine.

So a simpler Back implementation is to let the inference engine to exit in the same fashion as the Quit function. The value of the Return State gives us the goal at which the respondent pressed the 'Back' button. The question to restart the questionnaire at is the previous goal that displayed a user interface. This goal is identified firstly by gathering a list of all the responses entered in the replies database. This gives record of the order in which the goals were processed, as the replies are always asserted into the start of this database. The resulting list of goals is search through to find the first goal that displays a template. Until that goal is found, then the reply for each goal that is checked is removed from the database. The reply of the first goal with a template is also removed. The Questionnaire is then restarted. The inference engine quickly returns to the goal identified as having the template. This is because the inference engine finds the first set of goals already have replies, thus these goals do not need to be processed. This continues until it gets to the first goal without a reply, this is the identified goal. Figure 4.6 describes this procedure. 


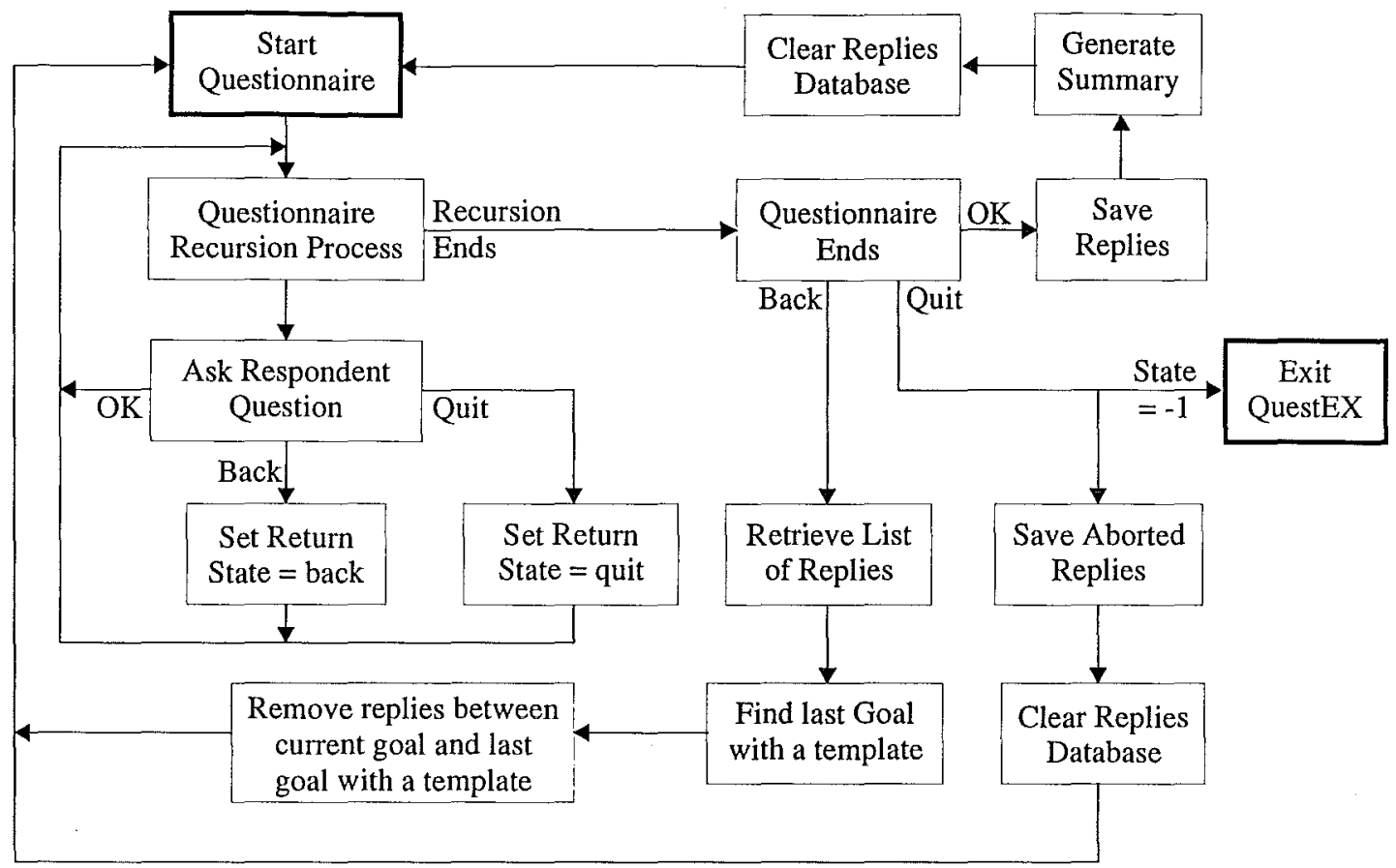

Figure 4.6 A flowchart description of the Back and Quit functions within the inference engine.

\subsection{CALCULATIONS AND CONDITIONS}

The calculations in QuestEX are performed by evaluating reverse polish notation (RPN) expressions. Each expression is stored within the goal's question object in two forms. The first form is the text representation of the expression, stored within the textlist field of the question object. It is not used in the evaluation of the expression. The second form is the reverse polish notation form, stored within the responselist field of the question object. To utilise the responselist field the domain declaration for the response type is:

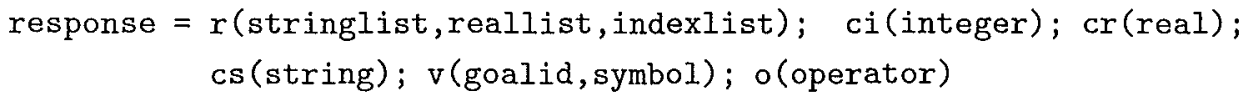

The $r$ (stringlist, reallist, indexlist) item is only used for the list based question responses, and is ignored in this context. The remaining objects in this declaration are used for the RPN expressions:

1. ci(integer). A constant index or integer value.

2. $\operatorname{cr}$ (real). A constant variable or real value.

3. cs (string). A constant string item. 
4. v(goalid, symbol). A goal reference. The goalid parameter denotes the goal to reference and the symbol parameter indicates the type of the reference, either an index, variable or string. The symbols used are "V", "I" and "S".

5. o(operator). An expression operator of some form.

The first four objects all obtain information from some source to be used in the expression. The source for the first three objects is the actual object, as the information is encoded as part for the object. The fourth object obtains information by referencing the result of another goal. The final object is used to perform mathematical operations.

The calculation responselist is processed in a recursive loop. A internal database calc (response) is used as a push down stack while the calculation is being evaluated. Each embedded constant from the first three constant calculation objects above is inserted into a response object and pushed onto the top of the calculation stack. For example, a ci(23) object would be pushed onto the stack as $r([],[],[23])$ and

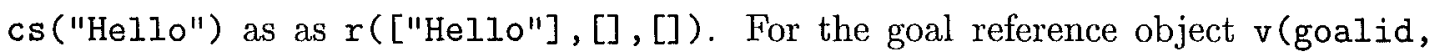
symbol) the questionnaire reply for that particular goal is retrieved. This reply is guaranteed to be available as the child goals of calculation are processed before the expression is evaluated. The referenced reply is then inserted into a response and pushed onto the calculation stack in the same way as the constant objects.

The expression operator o(operator) is handled differently as it provides the main functionality of the calculation module. The operator domain is defined as:

operator $=$ one_ary $($ symbol $) ;$ two_ary $($ symbol $) ;$ func $($ symbol $)$

The two ary (symbol) operator is used for operations that take two arguments. The func (symbol) is used for function operators. Currently there are no operators defined for the one_ary (symbol) operator as there are very few of these operators. One notable example here is the unary minus; currently the QuestED calculation parser is unable to differentiate between the binary and the unary minus so the unary minus operator has not been implemented. The symbol that both these operators contain is a string token which denotes the particular operation. Table 4.3 shows these text strings.

Table 4.3 The Calculation Operators

\begin{tabular}{|c|c|c|c|c|c|}
\hline \multicolumn{3}{|c|}{ Functions } & \multicolumn{2}{c|}{ Two-ary Operators } \\
\hline \hline SIN & EXP & ABS & + & AND & $=$ \\
COS & LN & SQRT & - & OR & $>$ \\
TAN & LOG 10 & TRUNC & $*$ & XOR & $<$ \\
ATAN & & ROUND & $/$ & & $<>$ \\
& & RANDOM & MOD & & $>=$ \\
& & & DIV & & $<=$ \\
\hline
\end{tabular}


At the RPN level there is no real difference between the func (symbol) and one_ary (symbol) operators. Currently they both pop a single response value off the stack, perform the operation and return the new value to the top of the stack. The only real difference is in the syntax of the expression, for example, "sin(5)" differs from "2 - -3" where the second minus is the unary minus.

The relational and Boolean operators work on the index field of a response item. The response $r([],[],[0])$ is defined as boolean false and $r([],[],[A])$, where $A$ $\neq 0$ is boolean true.

The arguments for the operator types are 'popped' off the calculation stack as required. After the operation has been completed, the resulting output response is pushed back onto the calculation stack. Function operators can take a list of variable (real) arguments and produce a list of variable output. This means that it can process the variable list response from a multi-select question in a batch form. The argument list is processed recursively and the output list is built up when coming out of the recursion. Each function symbol token is matched to a Prolog function pointer. This function pointer is then called to evaluate the desired function. The particular functions used were chosen from the standard Prolog mathematical functions. Each function is evaluated inside a 'trap' predicate. This catches any arithmetic overflow or division by zero exceptions. If an exception occurs then the function returns an empty response $r([],[],[])$. This empty response is caught at a higher level and the result of the entire calculation goal is an empty response with a false state.

The Two-ary operators are more complex. Whereas the function operators worked with variable arguments, the two-ary operators work with all three argument types. Also it is possible to mix types within an operation. For example it is permissible to add a index argument to a variable argument to produce a variable output. However it is not permissible to subtract a string argument from a variable argument. Table 4.4 shows the currently possible conversions between argument types for two-ary operations.

Table 4.4 The type conversions for Two-ary operations

\begin{tabular}{|c|c|c|}
\hline Argument A & Argument B & Output Type \\
\hline \hline Index & Index & Index \\
String & String & String \\
Variable & Variable & Variable \\
Variable & Index & Variable \\
Index & Variable & Variable \\
\hline
\end{tabular}

The steps then required to evaluate a two-ary operations are:

1. Decode input argument list.

2. Perform type conversions if necessary.

3. Count number of items in each list. 
4. Check argument list lengths.

5. Evaluate operation on the argument lists.

It necessary to determine the lengths of each list as there are four possibilities when performing the operations. An analogy is vector and scalar addition. For example the four possibilities for addition are:

1. Scalar addition, one item per list. $[\mathrm{A}]+[\mathrm{B}]=[\mathrm{C}]$.

2. Vector addition, equal number of items per list. $[\mathrm{A} 1, \mathrm{~A} 2]+[\mathrm{B} 1, \mathrm{~B} 2]=[\mathrm{C} 1, \mathrm{C} 2]$. Where $\mathrm{C} 1=\mathrm{A} 1+\mathrm{B} 1$ and $\mathrm{C} 2=\mathrm{A} 2+\mathrm{B} 2$.

3. Vector offset addition, one list has one item. $[\mathrm{A} 1, \mathrm{~A} 2, \mathrm{~A} 3]+[\mathrm{B} 1]=[\mathrm{C} 1, \mathrm{C} 2, \mathrm{C} 3]$. Where $\mathrm{C} 1=\mathrm{A} 1+\mathrm{B} 1, \mathrm{C} 2=\mathrm{A} 2+\mathrm{B} 1$ and $\mathrm{C} 3=\mathrm{A} 3+\mathrm{B} 1$.

4. Illegal operation. Lists do not contain the same number of items. [A1,A2] + $[\mathrm{B} 1, \mathrm{~B} 2, \mathrm{~B} 3]=$ ?

The argument lists are processed recursively in a manner suiting the lengths of the lists. Each two-ary token is matched to a Prolog function pointer in the same way as the function tokens. This function pointer is then used to evaluate the desired function. Again the particular functions used were chosen from the standard Prolog mathematical operators. Each operation is responsible for checking possible error conditions which may occur. For example, the integer division operator for strings returns an error. The whole list recursion routine is evaluated inside a 'trap' predicate. 'This catches any unexpected arithmetic exceptions. If an error or exceptions occur then the function returns an empty response $r([],[],[])$. This empty response is caught at a higher level and the result of the entire calculation goal is an empty response with a false state.

Once all the items in calculation responselist have been processed, the calculation has ended. If the calculation was well formed as an expression, there should be one remaining item on the calculation stack. This last item is the answer to calculation. If the answer is an empty response item such as $r([],[],[])$ then a calculation error has occurred and the calculation goal's state is set to false by the inference engine. Otherwise the answer calculation item is returned to the inference engine, which stores it as the questionnaire response to the current calculation goal.

Conditions are handled in much the same way as calculations. The main difference is that the condition responselist consists of three parts: the left hand side, the right hand side and the relation. The relation appears last in the list because of the reverse polish notation nature of the list. In QuestED the condition is entered as the three separate parts mentioned earlier. However once parsed into RPN form the three parts are concatenated into one condition list. The condition list is processed by passing it 
to the same RPN evaluator is the calculation list. The difference this time being that the last item in the calculation stack should be a index response. This is because a relationship operator was the last operator to be proceeded. If the index response is $r([],[],[0])$ then the condition has evaluated false, otherwise the condition is true. This result is passed back to the inference engine which stores it as the questionnaire response for the current condition goal.

\subsection{USER INTERFACE}

The User Interface in QuestEX was designed with the following aims in mind:

1. It should provide a sufficient variety of question types for a wide range of possible questionnaire types. The four question types implemented were Text entry, Numeric entry, Single selection list and Multiple selection list. The two information types, Group and Info, provide the ability to give information feedback to the respondent.

2. It should provide an author definable questionnaire interface for the respondents. Allowing the author to customise a questionnaire's user interface can create a better rapport between the respondent and the questionnaire administrator (QuestEX).

3. It requires Multimedia capabilities. Multimedia is becoming a major driving force in the acceptance of personal computers as a consumer product. Multimedia is seen as a contrast against the user-unfriendly nature of computers. It provides features to which a consumer can easily identify with.

The concept of a screen template was created to implement these aims. A template is a description of how a series of objects should appear on the screen. A number of these template objects are required for every template as they provide some basic questionnaire operations. For example each template must contain an 'OK' button as the respondent presses this button to indicate that they have finished answering the question. The other template objects are purely for decoration. For each question there could be any number of templates which could be used interchangeably without effecting the questionnaire operation, only the question appearance.

In QuestEX, nearly all the user interface functions are provided by the Template Manager. In the following sections the roles of templates and the Template Manger are described.

\subsubsection{Template Manager}

The Inference Engine calls the Template Manager whenever the current goal requires some interaction from the respondent. The Template Manager performs the following 
tasks:

1. Initialises the screen display for QuestEX.

2. Renders the template using the current goal information to a memory bitmap or metafile.

3. Opens appropriate multimedia devices for sound, music and animation events.

4. Displays the rendered template and enters an internal message loop.

5. Retrieves the respondent's replies.

6. Closes multimedia devices and cleans up any allocated resources.

The screen display initialisation is only performed once when QuestEX is started. The remaining four steps are handled by the XUI_AskQuestion predicate. The XUI_Askquestion predicate requires two input parameters, Qstobj and Templobj. The Qstobj object contains the question specific information for the current goal. The TemplObj object contains a complete description of the screen template. When the template is ended by the questionnaire respondent, the XUI_AskQuestion predicate returns two variables, Answer and State. The Answer variable contains a responselist structure which holds the respondent's reply. The State variable indicates how the template was ended, either by pressing the 'OK' button, 'Back' button or the 'Quit' button.

If the question has no template, as in the case of a hidden group goal, then the predicate returns immediately and the respondent sees nothing of the goal. Otherwise the question and template objects are stored globally in an internal database. This so they can be accessed when the private message loop is operating. The TMP_PrepareWindow sub-predicate then prepares and renders the template and TMP_ShowWindow displays the template on the screen. The private message loop is then entered. At this stage, the template has appeared on the screen and the respondent can interact with the template items, allowing them to enter a reply. Once the respondent has signalled the end of the template with one of the three buttons mentioned previously, the private message loop ends. The respondent's reply is then retrieved from the template and any resources associated with the template are freed. The XUI_AskQuestion predicate then ends returning the answer and state for that goal.

Each template has a number of properties. These are defined by the domain:

templateobj $=t$ (templateid, NameStr, goaltype, ModeInt, ErrorStr, objectlist)

The template properties defined by the templateobj domain are:

- templateid - An unique integer identifier used to identify individual templates. 
- NameStr - The text name of the template. It is defined by and is used to identify the template to the questionnaire author.

- goaltype - Indicates what goal type this template can be used for. A goal can only use a template which has the same goal type. The ' $g t_{-}$' prefix denotes a goal type constant.

- ModeInt - The template display mode. This can be either 'metafile' or 'bitmap'. The metafile mode is more efficient in terms of memory, however the time required to draw the template on the screen increases with the complexity of the template. The bitmap mode generates a complete screen bitmap in memory and always takes a constant amount of time to draw on the screen. The size of the off-screen bitmap increases with the screen resolution as does the time to draw the bitmap. The bitmap mode must be used if the template contains graphic images. The two modes are denoted by the tmp_bitmap and tmp_metafile constants.

- ErrorStr - This is the error message displayed to the respondent when a mistake is made when entering a reply. For example when a respondent presses the 'OK' button in a Single Select question with out selecting a response.

- objectlist - An ordered list of template objects. Each object represents a single part of the template user interface. The first item in the list is the first item to be drawn on the screen, therefore it is at the back of the template. The last item in the list therefore is on the top of the template. The child windows controls, buttons, listboxes and edit fields are by default on top of the template and so appear last in the objectlist.

There are sixteen different types of template objects. They are all represented by the object domain:

object $=$ obj(objid, objtype, rect, fnt, colour1, colour2, style, objextra)

objextra $=\operatorname{text}$ (string); $\operatorname{select}(\operatorname{col}$ lour); none

objectlist $=$ object*

The object properties defined by the object domain are:

- objid - A unique integer identifier to identify individual objects within a template.

- objtype - Indicates the type of the template object. The obj-denotes a object type constant. See table 4.5 for the full list of object types.

- rect - A standard rectangle structure which defines the size and location of the template object. The rectangle structure is defined as, rect $=\operatorname{rct}($ LeftInt, TopInt, RightInt, BottomInt). 
- fnt - A font structure which defines the font name and font size of the font used in the object. The font structure is defined as,

fnt $=f($ FontNameStr, FontSizeInt $)$.

- colour1 - This defines the primary colour of the object. The primary colour is normally the colour of an object's text, or solid fill.

- colour2 - This defines the secondary colour of the object. The secondary colour is not always used but it is normally the background colour of an object.

- style - The style parameter is used to define a series of styles for an object. Currently only the text based objects use the style parameter to set text alignment options.

- objextra - This parameter allows the inclusion of extra information relevant only to particular goal types. Mostly this parameter is left as 'none' however the miscellaneous text object and button objects use this field to store miscellaneous text and the button label. The list box object uses this field to store a third colour value. This colour value defines the background colour of a selected list item.

Table 4.5 gives a full breakdown of the different template object types and which parameters are used in the object structure. The Mode column indicates whether the object is drawn as a static graphic or created as a window. A window based object is able to redraw itself dynamically and respondent to events in the system.

Table 4.5 The attributcs of each template object type.

\begin{tabular}{|c|c|c|c|c|c|c|c|}
\hline objtype & rect & fnt & colour1 & colour2 & style & objextra & Mode \\
\hline obj_backgnd & - & - & BackGnd & - & - & none & Gfx \\
obj_questext & Yes & Yes & Text & - & Yes & none & Gfx \\
obj_helptext & Yes & Yes & Text & - & Yes & none & Gfx \\
obj_titletext & Yes & Yes & Text & - & Yes & none & Gfx \\
obj_misctext & Yes & Yes & Text & - & Yes & text(Misc) & Gfx \\
obj_okbutt & Yes & Yes & Text & Face & - & text(Label) & Win \\
obj_backbutt & Yes & Yes & Text & Face & - & text(Label) & Win \\
obj_quitbutt & Yes & Yes & Text & Face & - & text(Label) & Win \\
obj_helpbutt & Yes & Yes & Text & Face & - & text(Label) & Win \\
obj_editbox & Yes & Yes & Text & BackGnd & - & none & Win \\
obj_listbox & Yes & Yes & Text & BackGnd & - & select(Clr) & Win \\
obj_line & Yes & - & Line & - & - & none & Gfx \\
obj_fillrect & Yes & - & Fill & Border & - & none & Gfx \\
obj_ellipse & Yes & - & Fill & Border & - & none & Gfx \\
obj_statrect & Yes & - & - & - & - & none & Gfx \\
obj_animrect & Yes & - & - & - & - & none & Win \\
\hline
\end{tabular}




\subsubsection{Template Initialisation}

The template manager is initialised immediately after a questionnaire has been loaded. At this stage nothing has been displayed on the screen. There are four tasks carried out when the template manager is initialised:

1. The physical screen size in pixels is obtained from the system. This is then stored for later reference. These sizes are most important as each template covers the whole screen, and all objects within the template are scaled within these values. This makes each template independent of the actual screen resolution.

2. A Window Class is created. Before creating a window to display the templates on, a window class must first be registered. The windows class defines a number of fundamental properties that every window based on that class possesses. The most important of the properties is a function pointer to that class' window procedure. The window procedure is a function that the operating system calls whenever there is an event or message for that window.

3. A Template window is created based on the window class that has just been registered. The template window is created as a child of the application window. As the application is currently hidden when the Template manager is initialised, the template window does not yet appear on the screen.

4. A series of child windows of the template window are created. These are the template buttons. The template buttons always occur in every template, although they may not be visible. They are therefore created once when the template is initialised. When a new template is rendered, the buttons are moved to the new position defined in the template. This means the buttons are re-used in every template, and are only destroyed when the QuestEX ends.

\subsubsection{Template Rendering}

To render the template a memory Device Context must be created. Windows uses a device context to represent a physical device. This physical device may be the screen, a printer, a bitmap or a metafile for example. Each device context contains a large set of attributes which describe the state or context of the device. Examples of these attributes are the font, pen, brush, mapping mode, extents, drawing mode and so on. QuestEX offers two template display modes: metafile and bitmap. These two modes require the creation of different device contexts, namely the memory bitmap and metafile device context. Once a device context has been created, it can be treated the same regardless of which method was used to create it. Two steps remain in initialising the device context, first an information context is created, and the coordinate system must be setup in both the device context and the information context. An information 
context contains the same attributes as the device context, except that it can not be written to, or drawn on. It is however useful for obtaining information such as text widths which can not be obtained from a metafile context. The same settings are maintained for both the device context and the information context to ensure consistency between them.

The device context mapping mode is set to the anisotropic mapping mode. This means the logical coordinate system for the device context is 'stretched' to fit the physical coordinate system. The logical coordinate system is set to the constants tmp_xsize,tmp_ysize. These constants are defined as 640 and 480 respectively, this is the size of the lowest screen resolution Windows supports. The physical coordinate system is set to $\mathrm{Cx}$ and $\mathrm{Cy}$. These being in the current screen resolution. So, irrespective of the current screen resolution, the logical coordinate system is defined by the constants tmp_xsize, tmp_ysize. The position and size of all the template objects lie within these two constants.

To render the template objects into the device context, the object list from the templateobj is recursively processed an object at a time. The TMP DrawObject predicate is called for each object. This predicate contains a clause for each of the template object types. The simplest clause is the obj_backgnd clause, it draws the template background. As indicated by Table 4.5 the colour 1 field of the template object contains the background colour value BackGnd. To draw the background solid brush is created using the BackGnd variable. The Windows FillRect function then paints a rectangle the size of the screen and using the coloured brush.

The obj_ellipse clause which draws an ellipse on the template is slightly more complex. Both the colour1 and colour2 fields of the template object are meaningful this time. colour1 represents the fill colour of the ellipse and colour2 represents the outline colour of the ellipse. The logical size of the ellipse is contained in the Rect field of the template object. Brush and pen objects are created using colour 1 and colour2. When the new brush and pen objects are selected into the device context, the old object handles are saved. The Windows Ellispe function then draws the ellipse into the device context using the logical size variable Rect. Finally the old brush and pen objects are selected back into the device context and the newer objects are freed from memory.

All the simple graphical objects do much the same thing as the ellipse clause. The window based objects however are different. The position of a child window relative to its parent window is given in physical coordinates, not logical coordinates. The information context created earlier is used to transform the logical coordinates back to physical coordinates. The object can then be accurately positioned within the template. The back, quit and help buttons are optional for each goal. The options for these buttons is stored in the goal's question object textlist field. Regardless of the state 
of the option, the position of the button is calculated, and a font object based on the object's fnt field is created. Most of the object information is then stored in a global database. The Window based buttons are all 'owner draw', meaning that QuestEX is responsible for drawing the button, not Windows. Consequently the object information must be accessible during the message loop as the owner draw events occur as Windows messages. A global database is the only suitable method available in Prolog for storing global information. Each button window is then moved to its new physical position and the TMP_ShowWindow predicate shows or hides the it depending on the value of the option variable.

The two response objects, the edit field and the listbox, are created anew for each template. This is because it is not possible to change the operation of these controls once created. For example, it is not possible to change a list box from single selection mode to extended selection mode once created. If one of these objects has been created, then it is destroyed when each template's resources are freed. Compare this to the four button objects which are only created once and are re-used in all the templates.

Once the template has been completely rendered, the memory device context is closed giving either a bitmap or a metafile handle. This handle refers to a Graphics Device Interface (GDI) object. This GDI object contains the memory representation of the template. The object handle stored in a global internal database until it is required when drawing the template on the screen.

The help text object is handled differently from all the other template objects. This is because the help text object effectively defines a dynamic place holder while all the other non window based template objects are static, meaning that all they do not change once that template has been rendered. However the contents of the help text object can change depending on the actions of the respondent. The following messages can appear in the help text object:

1. The help text string defined in for a particular goal. This stored in a goal's question object textlist field. This message would be used to give help information relevant to the current question.

2. The template error message. This message provides some help on the usage of the template. It is stored in the template's ErrorStr field. For example, pressing the 'OK' button in a numeric entry question without entering an answer.

3. The less than minimum error message (numeric entry only). This message is displayed when the respondent enters a number less than the defined minimum for the question. This consists of the template error string ErrorStr plus "The number must be greater than \%." where the \% symbol contains the defined mimimum. 
4. The greater than maximum error message (numeric entry only). As for the previous message but with the defined maximum.

Each of these messages are created in their own mini template metafile which has the same coordinate system as the main template. The metafile handles for these templates are stored in an internal database. A message can be displayed by playing the desired metafile after the main template has been drawn on the screen. The TMP_ShowHelp predicate sets the current help message and forces a screen redraw which redraws then main template and then the current help message. See section 4.5 .5 for further information. When the template resources are freed, all the help message metafiles are freed as well. See Figure 4.7 for an example of a template.

\section{Teenigge Heslith \&? Fitriess Q! Lestibrirlaire}

This is a confidential questionnaire and no information on it will be passed on to anyone else, including parents or other members of your family.

The aims of this questionnaire are to :-

a) detect any problems that you may have and wish to discuss further, either with the doctor or nurse.

b) improve the care that your doctor and nurse can give you and young people generally

Press the Green 'Start' button to begin the questionnaire.

\section{Quit Start

Figure 4.7 An example of a Introduction goal group template

\subsubsection{Queuing Multimedia Events}

There are four different types of Multimedia events. These are sequenced MIDI music, digital WAVE audio, AVI video animations and static graphic images. The first three types are provided by the Windows Media Control Interface (MCI) services. The MCI services allow installable multimedia drivers to be added to windows. The interface to each multimedia driver is defined by the MCI services which provides a simple and consistent interface to a wide variety of drivers. The MCI services defines both a high level and a low level command interface. The low level command interface allows direct manipulation of WAVE and MIDI files. The high level interface provides a simple set 
of commands for opening, playing and closing multimedia files. The mciSendString function provides a simple and convenient method for sending MCI commands. As a parameter it takes a string containing the command and any associated argument. The MCI interprets this string and executes the command. A return code indicates the success of the command. If the command was not successful, then calling the mciGetErrorString returns a text error message indicating the problem. This text error message is written to the questionnaire log file.

To play an MCI multimedia file, the following steps are taken:

1. Open the file for the appropriate device. The command string to do this is created using the prolog string format predicate:

format(MCIStr,"open \% type waveaudio alias sound ",SoundFile).

The SoundFile string variable contains the filename to open. 'type waveaudio' indicates the MCI device to open, and 'alias sound' gives the waveaudio device an alias which is used to refer to the device later. Each device is opened while the template is being rendered.

2. Play each device. This starts the device playing. At this stage the template is in the process of being displayed on the screen. The command string for this is:

mciSendString("play sound notify", "", 0, TmpWnd)

This plays the device with the alias sound. 'notify' indicates that when the device has finished playing the file, a notification should be sent to the template window denoted by TmpWnd.

3. When a notification message is sent to the window TmpWnd a check is made to determine which device sent the message. Once the device is known, a check is made to see whether this device should then repeat or loop the file. The Loop option is originally stored in the flag list field of the multimedia item structure. The multimedia item structure is stored in the MMList field of the questionnaire object. The Loop option is stored in a temporary database until the notification message is received. If the Loop option is set then, the following command strings are sent:

mciSendString("seek sound to start", "1, 0, 0), mciSendString ("play sound notify", " , 0, TmpWnd)

The first command returns the device to the start of the file, and the second plays the device again. The looping for the sequenced music is more complicated, see below for more details.

4. Finally when the template is finished, the devices must be closed. The is done with: 
mciSendString("close sound", "1, 0,0),

This simply closes the device called sound.

The loop notification for the sequenced music device is more complex as it deals with a playlist of files. When a goal sets up a music play list, it initialises a special temporary database:

music (MusicGoalType, GoalId, DeviceId, PlayList, ToPlayList, MusicRandom)

The most important fields in this database are the final three. The PlayList contains the complete list of tracks in the current playlist. The ToPlayList initially starts out the same as PlayList; however as each new track is played, the track is removed from the ToPlayList. When the ToPlayList is finally empty, it is re-initialised with the PlayList. The MusicRandom flag indicates whether the next track to play should be the first in the ToPlayList, or a random choice.

The graphic images are handled differently to the three other Multimedia events. The MCI interface provides no support for graphic images. The Windows Graphics Device Interface (GDI) does however provide a set of low level functions for manipulating bitmap graphics and graphic primatives. There are two main types of images commonly used in Windows, bitmap images and metafile images. Bitmap images are normally stored in a file as device independent bitmaps (DIB). These DIB images contain a colour palette and a bitmap of palette indices. Once a DIB is loaded, it is then converted into a device dependent bitmap and can be displayed on screen.

Metafile images consist of a list of graphic primitives which are stored in a binary form. There are a number of simple Windows functions to create, open and play metafile images. However basic metafile images contain no information about the actual size of the image, in image coordinates. So while it is easy to scale a metafile image, it is difficult to accurately control the scaling. Placeable metafiles include a small header block which contains size information, however it is not compatible with the Windows functions to manipulate metafiles. This can be handled by stripping off the header, but this is difficult in Prolog.

As Prolog is not as suitable for handling these image types, a $\mathrm{C}$ or $\mathrm{C}++$ library would be required. A suitable library was found in Borland C +4 Developer's Guide by Nabajyoti Barkakati (Barkakati, 1994). It provides facilities for loading Windows Bitmap (BMP), Zsoft's PC PaintBrush (PCX), Targa (TGA), Tagged Image File Format (TIFF) and Graphics Interchange Format (GIF) images. The library makes use of the Borland Application Framework library called ObjectWindows Library or OWL. While convenient, the OWL library increased the size of the final dynamic link library. So all the OWL code was replaced with standard Window API calls. This resulted in a saving of around $100 \mathrm{Kbytes}$ for the dynamic link library.

An important requirement of the image library is the speed of image decoding. While rendering a template it is important that the respondent doesn't have to wait 
more than a second. This rules out those image formats that use compression schemes, i.e. TIFF and GIF. Another restriction is the quality of the support for the image format. The Targa file format is capable of storing 24bit graphic images, however the image library only supported 8bit images.

The BMP format implemented 2,4 and 8 bit mode and the PCX implemented a 24bit mode. This gave sufficient coverage of the common colour depths. However the image library did not support the Windows metafile format (WMF). Due the object orientated approach to the image library it was possible to define the sub-image source code for loading and displaying both standard metafile images and placeable metafile images.

To further improve the performance of the image library, an image cache was devised. The image manager allows the up to 15 images be loaded at one time. Given the filename of an image, the image manager identifies the format of the image and loads it accordingly. If the image is already in the cache, then the load function returns immediately. Once loaded the image is converted to DDB and stored in the cache. When the image is displayed, the DDB from the cache is displayed onto a supplied device context. The exception here is for metafiles which are not converted to a DDB and are consequently drawn using the standard Windows function PlayMetafile. The image cache is normally cleared after each template.

\subsubsection{Display Template}

Displaying the template once it has been rendered and the multimedia objects have been queued is simple. This task consists of two steps:

1. Play any queued multimedia events. This maybe either a movie or a sound clip.

2. Invalidate the template window. Making any window or region of the screen invalid forces Window to update that area as soon as possible. Thus the template is redrawn.

Immediately after the template window has been updated, QuestEX enters a private own message loop. In a normal Windows application, the application message loop would be used to receive and dispatch window messages. Although QuestEX has a standard application message loop it is better to use a private message loop while a question is being asked. In this way only those messages that are directly relevant to QuestEX need be processed. Also it is possible to start the private message loop and then end it when the respondent has entered a reply. It is not possible to end the application message loop without ending the application.

There are a number of important messages that are processed while the private message loop is active These messages are trapped within the template window procedure: 
- wm_paint. This message indicates that the template window should draw itself. This message occurs when the template window has been invalidated, for example when the template is first displayed. In response to this message, the template object handle stored within a global internal database is retrieved. This object handle is then used to transfer the template from memory to the screen. The current help message as set by the TMP_ShowHelp predicate is retrieved and the associated help message metafile is played on to the screen.

- wm_ctlcolor. This message is processed to change the colour of any text edit fields or list box field with in the template. All colour attributes of the control can be changed, for instance the text colour and background colour of a edit field. The background colour of a list box can be changed, however the owner draw message is responsible for drawing the text of each list box item.

- wm_keydown. This message is handled to provide a keyboard interface for the four templates button. The 'B', ' $Q$ ', ' $\mathrm{H}$ ' and enter keys generate events for the Back, Quit, Help and OK buttons respectively.

- wm_syskeydown. The message is 'eaten' to stop any system key events from occurring. For instance it blocks the 'Alt-F4' key press which would normally close the application.

- wm_command. The template message receives a wm_command when one of the four templates button are pressed.

- wm_drawitem. This message indicates that one of the template window's child controls needs to be redrawn. The four buttons and the list box controls all have the 'owner draw' attribute set to allow different fonts and colour to be used within them. The information required to draw a button or a list box item is stored in a global internal database when the item was rendered.

- mm_mcinotify. This message is received when a multimedia device has finished playing. See 4.5 .4 for more details.

- wm_timer. The timer message is used for the popup help message. A question help message can be displayed after a set period of time.

\subsubsection{Closing the Template}

There are three different methods for ending the template, each method is associated with three template buttons: 'OK', 'Back' and 'Quit'. Each method makes use of an internal database answer (response, State) for storing the result of the template. The answer database contains a response item for holding the respondent's entry for the current question. The goalid item holds the return state of the template. This 
is defined to be Return State $=$ qs_ok $=0$ if the respondent ended the template by pressing 'OK', Return State > qs_ok = current goal id if the 'Back' button was pressed and Return State < qs_ok = - current goal id if the 'Quit' button was pressed.

The 'Back' method for ending the template is the simplest. The answer database is set as answer $(r([],[],[])$, Goalid). The private message is terminated by posting the wm endtemplate message into the private message loop queue. This is a window message defined by QuestEX for its own use. It is the only message that stops the private message loop. Once the message loop has ended, control returns to the last sub-predicate in the XUI_AskQuestion predicate. This is the TMP_ClearWindow subpredicate, it's task is to clean up the resources used in the template process. This involves:

1. Closing and freeing any multimedia devices and files.

2. Freeing font and brush resources used for the owner draw controls.

3. Freeing the metafile help messages resources.

4. Finally freeing the memory template bitmap or metafile resource.

The responselist Answer and the State variables stored in the answer database are retracted and returned as output arguments in the XUI_AskQuestion predicate.

The 'Quit' method for ending the template contains two separate cases, which are:

1. The current goalid is 1 . This means the respondent has pressed the 'Quit' whist in the questionnaire introduction goal. The questionnaire password option is stored in the questionnaire options. This is obtained, from which there are two further cases:

- If the questionnaire password option is enabled, then the password is extracted from the questionnaire options and the Password Exit dialog box is displayed. See Figure 4.8. The password must be typed correctly before pressing the 'OK' button. The password check is not case sensitive. If the password is incorrect or the respondent pressed 'Cancel' then control returns to the current template.

- If the password is disabled, then a simple Yes/No dialog box prompts the respondent for confirmation for exiting QuestEX. If the respondent choose 'No' then control returns to the current template.

2. Otherwise the respondent has pressed the 'Quit' from some other goal. A simple Yes/No dialog box prompts the respondent for confirmation for ending the current questionnaire session. If the respondent chose ' $\mathrm{No}$ ' then control returns to the current template. 


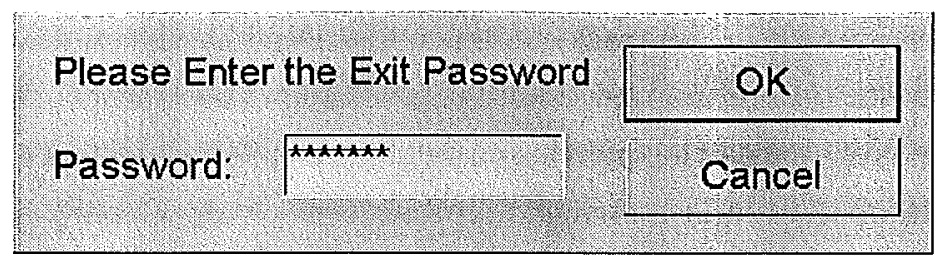

Figure 4.8 The exit password dialog box is QuestEX.

In both cases if the respondent chooses to quit then the answer database is set as answer $(r([],[],[])$, -Goalid). The private message loop is terminated by posting the wm_endtemplate message into the application message queue. The template cleanup proceeds is the same fashion as for the 'Back' case.

The 'OK' method for ending the template involves retrieving the respondent's answer from the template reply fields. Each of the different question types has it's own method for obtaining this information and checking its validity. For a numeric entry question, the reply is first converted to a number and then checked to see if it lies between the optionally specified minimum and maximum values for the question. If any of these tests fail then the appropriate help message is displayed to the respondent and the template is not terminated. Likewise for a text entry question a help message is displayed if the respondent does not enter a reply and the same occurs for a single select question if an item is not selected. If the reply is valid then it is stored with the answer database and the private message loop is ended.

\subsection{QUESTIONNAIRE SUMMARIES}

A questionnaire summary is a text based form which contains a formatted text report of a questionnaire session. A questionnaire session is a single run through of a questionnaire by a respondent. For each questionnaire any number of summaries maybe defined. The actual context of each summary is defined by the questionnaire author. A 'tracker' file keeps track of the individual questionnaire sessions. The summary generation for a questionnaire session revolves around the following sequence of tasks:

1. Obtaining the list of Summaries to generate.

2. Retrieving the session number from the tracker file and calculating the next session number.

3. Processing each summary.

4. Saving the new session list to the tracker file.

The list of summaries is obtained from the questionnaire fileinfo structure which the Database manager maintains. This structure contains a list of all the summaries

Whathenth 
defined for the questionnaire. The tracker file is maintained by the summary generator to distinguish between individual questionnaire sessions. The tracker file is identified by having the same filename as the questionnaire file, but having the filename extension 'trk'. The contents of the file is a string representation of an integer list. The list is ordered with the most recent session first. For example, after five questionnaire sessions the file would contain the string, "[5,4,3,2,1]". This format was chosen as it is simple to convert between Prolog terms and strings read from files. The new session number for the current session is calculated by incrementing the greatest session number. It is then appended to the session list and saved back to the tracker file.

\subsubsection{Summary Processing}

Each individual summary is stored in the following structure:

summaryobj = s(summaryid, Name, Style, sumprint, sumfile, sumentrylist)

The most important fields in the summaryobj object are the final three. The sumentrylist field is a list of the individual summary entries. There is a summary entry for each questionnaire goal. The sumprint and sumfile objects contain the output characteristics for the summary object. The following set of tasks are followed for each summary:

1. Obtain the Summary object from the Database manager.

2. Both the sumfile and sumprint structures contain a boolean Enabled option. This option indicates whether the summary is saved to a file or printed. Both the options for these structures are checked, if they are both False, then this summary is not saved or displayed in any form. Therefore the summary processing for this summary is aborted and the next summary is then processed.

sumprint $=\operatorname{sp}($ Enabled, Stretch, FontName, FontSize)

sumfile $=$ sf(Enabled, Directory, Filename)

3. Otherwise the summary is created with the summary output saved in a temporary file. See section 4.6.2 for further details on the summary generation. The temporary filename is obtained using the Windows API function GetTempFileName. This ensures a unique filename in a designated temporary directory.

4. A test is made on the size of the temporary file. If it is empty then the remainder of the processing for this summary object is aborted and the next summary is then processed. This situation may happen if a file error occurs during the summary creation for example. 
5. The information in the sumfile object is then used to write the summary out to a file. If however the Enabled field is false, then this operation is skipped. Otherwise a filename is constructed from the Directory and Filename fields and the questionnaire session number. The Directory term refers to a subdirectory of the questionnaire directory, that is the directory of the main questionaire file. The Filename provides the first five characters of the file name, and the session number the last three. For example, if the questionnaire directory is ' $\mathrm{C}: \backslash$ quest $\backslash$ ', the Directory field is 'smoke', the Filename field is 'file' and the session number is 5 , then the full filename is ' $\mathrm{C}: \backslash$ quest $\backslash$ smoke $\backslash$ file005.txt'.

6. The sumprint object is used to print the summary out to a printer. See section 4.6.3.

7. The temporary file is then deleted from the system temporary directory.

\subsubsection{Summary Generation}

The actual summary generation revolves around processing each of the entries in the sumentrylist of the summaryobj. The sumentrylist is a list of summary entries, which is defined as:

sumentry $=$ se (goalid, Enabled, Type, Description, Style)

The goalid field relates directly to the associated questionnaire goal for this entry. The summarylist contains an entry for every goal in the questionnaire. The Enabled field indicates if the entry is to be actually used within the summary. If the Enabled field is false then this entry does not appear in the summary. While having an entry for every goal is excessive, it means that an entry removed from the summary retains its Description and Style as the only field that is changed is the Enabled field. The Type field indicates the type of the goal reference. This is essentially the same as for the goal references in the main questionnaire, "S" for string, "I" for index and "V" for variable. The Description field contains the text that appears in the summary. In most cases the Description contains a " ' character. This indicates where the formatted goal response is inserted. For example, say an entry has a Description field "Age of Patient = - years", a Style of "V", and an associated response of $r([,,[23.5],[])$, then formatted summary entry is "Age of Patient $=23.5$ years"

Each summary is generated as a 80 column text file. Therefore consideration must be given to the width and positioning of each summary entry within the text file. For instance long lines must be wrapped on to the next line. However this allows a number of formatting possibilities for each entry, for example tabs, blank line, centred lines and right justified lines. To facilitate this, a column counter is maintained. This column counter holds the end position of the last entry in the file. From this position, 
the formatting for the next entry can be calculated. The Style field in the sumentry object indicates the formatting for the entry. Table 4.6 shows all the possible formatting options,

Table 4.6 The different summary entry formatting options.

\begin{tabular}{|c|c|c|}
\hline Identifier & Description & Counter \\
\hline sumf none & $\begin{array}{l}\text { Performs no formatting, next entry follows } \\
\text { immediately. }\end{array}$ & Adds entry length \\
\hline sumf newline & $\begin{array}{l}\text { Inserts a new line, next entry follows on } \\
\text { the next line. }\end{array}$ & Reset to 1 \\
\hline sumf_blankline & Inserts two new lines, leaving a blank line. & Reset to 1 \\
\hline sumf_tab & $\begin{array}{l}\text { Inserts spaces up to the next } 8 \text { character } \\
\text { boundary. }\end{array}$ & Adds up to 8 \\
\hline sumf halftab & $\begin{array}{l}\text { Inserts spaces up to the next } 40 \text { character } \\
\text { boundary. }\end{array}$ & Adds up to 40 \\
\hline sumf_centered & $\begin{array}{l}\text { Inserts spaces before the entry to center } \\
\text { the entry on the line, next entry follows } \\
\text { on next line. }\end{array}$ & Reset to 1 \\
\hline sumf right & $\begin{array}{l}\text { Inserts spaces before the entry to right jus- } \\
\text { tify the entry on the line, next entry fol- } \\
\text { lows on next line. }\end{array}$ & Reset to 1 \\
\hline
\end{tabular}

In all cases if the column counter exceeds 80 characters, then line current entry is broken at the first space from the right hand line of the end entry that is within the 80 character line length. The entry then continues on the next line.

The actual sequence of tasks to process each summary entry is:

1. Gets the reply from the database manager for the referenced goal

2. If the goal state is false, then skip this entry and do the next.

3. If the goal response if empty, that is $[r([],[],[])]$, then the respondent has selected none of the items in a multi select question. As this tells us no information, then skip this entry and do the next.

4. Make the a text string from the goal response based on the entries' Type field.

5. Insert the new response text string into the entries' Description string field.

6. Apply the formatting Style to the new Description string to produce the Summary string. Update the column counter accordingly.

7. Write the Summary string out to the temporary file.

\subsubsection{Summary Printing}

The QuestEX printer module is initialised when QuestEX starts. This allows the author to choose the output printer and the printer setup from the Open questionnaire dialog 
box. These settings are saved until it is time to print a summary. If the author did not choose a printer at the initial stage, then the default system printer is used.

Once the output printer settings are in order a Windows device context is obtained based on that printer. From the device context the physical printer page information can be obtained. This information is used to format the summary page layout. Table 4.7 shows all the information required to format the page.

Table 4.7 The information required to format a summary page

\begin{tabular}{|c|c|c|}
\hline Quantity & How obtained & Description \\
\hline SxPage & GetDeviceCaps & Width of DC in pixels. \\
\hline SyPage & GetDeviceCaps & Height of DC in pixels. \\
\hline SxInch & GetDeviceCaps & Number of pixels per logical inch across the DC. \\
\hline SyInch & GetDeviceCaps & Number of pixels per logical inch down the DC. \\
\hline SxOff set & Printer Escape & $\begin{array}{l}\text { Horizontal offset from top-left corner to the actual } \\
\text { printing area. }\end{array}$ \\
\hline SyOffset & Printer Escape & $\begin{array}{l}\text { Vertical offset from top-left corner to the actual } \\
\text { printing area. }\end{array}$ \\
\hline OrgX & Calculation & $\begin{array}{l}\text { OrgX }=\text { (prnt xmargin*SxInch) - SxOffset. } \\
\text { The Printable origin. prnt xmargin is a constant } \\
\text { which defines the printing margin in inches. }\end{array}$ \\
\hline OrgY & Calculation & $\begin{array}{l}\text { OrgY = (prnt_ymargin*SyInch) - SyOffset. } \\
\text { The Printable origin prnt_ymargin is a constant } \\
\text { which defines the printing margin in inches. }\end{array}$ \\
\hline Width & Calculation & $\begin{array}{l}\text { Width }=\text { SxPage }-2 * \text { OrgX. } \\
\text { The printable page width. }\end{array}$ \\
\hline Height & Calculation & $\begin{array}{l}\text { Height }=\text { SyPage }-2 * 0 r g Y . \\
\text { The printable page height. }\end{array}$ \\
\hline CyChar & GetTextMetrics & Height of a character cell. \\
\hline CxChar & GetTextMetrics & Average character width. \\
\hline MaxLines & Calculation & $\begin{array}{l}\text { MaxLines }=\text { Height div CyChar. } \\
\text { Number of lines in a page. }\end{array}$ \\
\hline
\end{tabular}

The CxChar and CyChar variables are obtained once the desired printer font has been selected into the printer device context. The font originally comes from the sumprint object presented in section 4.6.1. This object contains three fields related to the printer font:

1. FontName. This is the face name of the font.

2. FontSize. This size of the font in points.

3. Stretch. This indicates whether the font is stretched to fit the page. If this field is false, then the values for FontName and FontSize are used to generate the font. If it is true, then the FontSize field is ignored. The actual font width is calculated so that 80 characters fit across the page. The font height is also recalculated so the font maintains the correct aspect ratio. 
Setting the Stretch field to true is useful with fast graphics printers, such as a Laser printer. Laser printers can print stretched True-Type fonts quickly and easily. For dot-matrix printers it is better to choose a printer font which is not stretched, rather than a True-Type font. Once the font has been selected into the device context the temporary file containing the summary can be printed. To do this it is simply a case of reading each line from the file and drawing it onto the device context. A counter is used to record the number of lines printed and to provide the vertical coordinate of each line of text. When the counter reaches the MaxLines value, it is reset to zero and a 'NewFrame' printer escape is sent to start a new page. This continues until the entire file has been printed.

\subsection{SUMMARY}

In summary, the QuestEX program consists of four main components, these are:

1. Inference engine. This controls the interpretation and execution of each questionnaire.

2. Questionnaire database manager. This manages the questionnaire structure information and the resulting replies from the respondents.

3. Template manager. This interprets the questionnaire user interface descriptions and controls the interaction with the respondents.

4. Summary manager. This processes the respondent's replies into a form described by the questionnaire author.

Each of these components manages the manner in which QuestEX interacts with the questionnaire respondents. The questionnaire file describes how each component should behave and consequently governs this interaction. The highly organised structure of the questionnaire file can not be interpreted manually. It is the task of the questionnaire editor, QuestED, to present this structure to the author in a comprehendible fashion. The implementation details of how QuestED achieves this is described in the following chapter. 


\section{Chapter 5}

\section{QUESTED IMPLEMENTATION}

QuestED is the name of the program that implements the questionnaire editing functions for the Information Driven flow model discussed in section 3.4. QuestED also provides functions for editing the questionnaire appearance and questionnaire summaries. This chapter presents the implementation of these functions within QuestED. The reply and summary processing functions of the QuestView program are also briefly described.

QuestED has been implemented using the new Visual Prolog Interface (VPI) from PDC Prolog. At this stage, the Visual Prolog compiler is still in beta testing. This version consists of a integrated development environment (IDE) and a VPI library. The IDE provides all the facilities for creating Microsoft Windows based projects, including: a syntax highlighting editor, dialog box editor, icon and bitmap editor and hypertext help file editor. The beta release 3 version was initially evaluated in March 1995. While the potential was seen in this version, it was not stable enough as an integrated development environment or as a class library. Beta release 4 arrived in April and was seen as stable enough to use. It also contained a series of VPI 'packages' which provided additional functionality, but which were undocumented. These packages provide facilities for drawing hierarchical trees, drawing context sensitive toolbars, managing dialog boxes and embedding syntax highlighting and hypertext text editors within an application. The tree package was seen useful in the representation of the questionnaire structure.

QuestED consists of the four major components, these are:

1. Database Manager. The Database Manager module provides access to the questionnaire file. It manages the information within the questionnaire file for the other components in QuestED through a series of functions.

2. Questionnaire Editor. The Questionnaire Editor module allows the author to create and manipulation the content and structure of a questionnaire.

3. Template Editor. The Template Editor module allows the author the questionnaire's appearance. This may by done by modifying the existing questionnaire 
user interface or by creating an entirely new look.

4. Summary Editor. The Summary Editor module allows the author to create customised reports. Any number of summaries can be defined for different purposes.

The following sections describes each of these components in greater detail.

\subsection{INITIALISATION}

QuestED has one main initialisation phase. The VPI framework performs some of the initialisation such as creation of the main window and its associated toolbars and menus. Once the main window has been created the following initialisation steps are performed:

1. Initialise the questionnaire editor's user interface

2. Initialise the template editor

3. Initialise the database manager.

4. Update the QuestED toolbar and menu.

The first step of initialising the questionnaire editor's user interface involves a number of tasks. Firstly a number of initialisation options are loaded from QuestED's private initialisation file which resides in the Windows directory. These options are used during the creation of the questionnaire tree view window which follows and are discussed further in section 5.3.1. Once the tree view has been created the first property panel is created and positioned inside the QuestED main window and next the tree view window. A number of global flags relating to the questionnaire user interface are set up at this stage.

The initialisation of the template editor is comparatively very simple. It involves the initialisation of a set of global flags for the template module. This step is very simple as the template editor is created anew each time the author edits a template, so much of the initialisation for the template editor occurs when template view window is created.

The database manager is also very simple as much of the real database initialisation occurs when a questionnaire is actually created or loaded. Again the database manager simply initialises a set of global flags for the module. Note that in each of the above three initialisation cases, each set of global flags is only global to the module in which they reside. There is no true 'global' information in QuestED as this does not conform to the principle of data hiding. 


\subsection{DATABASE MANAGEMENT}

While the database manager in QuestEX has the relatively simple tasks of opening a questionnaire file and reading the questionnaire objects when required, QuestED's database manager has a more complex role. QuestED has to deal with the loading and saving of database information temporarily stored within QuestED. Whereas QuestEX provides the single service of obtaining questionnaire information from the questionnaire file, QuestED provides three services. These are: obtaining questionnaire information, modifying questionnaire information and managing the questionnaire file.

As discussed in section 4.2 the questionnaire file is a Prolog external database which consists of a number of object chains. The information within each chain is accessed through a binary search tree which associates object identifiers with the object's position within the chain. The QuestED database manager provides an interface to the questionnaire file for other modules within QuestED. It does this by reading in all the questionnaire objects from the external questionnaire file into internal storage. Access to the internal questionnaire object store is through a set of functions which are presented later.

There are two methods for storing the questionnaire information, one is to use a Prolog external database which resides in memory; this is similar to how QuestEX manages the questionnaire file. The other method is to use Prolog internal databases. This second method has been implemented with an aim of using the first method in a future version of QuestED. The internal database method has the advantage of simplicity. Objects can be stored and removed from an internal database with Prolog assert and retract functions. Searching an internal database is done through matching and unification as the internal database appears as a predicate. A disadvantage is that the internal databases are slower when searching for an object to match. Simple tests have shown the binary tree/external database method is faster when generating and searching the questionnaire tree. However it is also more complex to implement.

The database manager handles the questionnaire file in a fashion seen in most Windows applications. The following functions implement the options found normally within the application file menu:

- DBD_NewFile. This function creates a new empty questionnaire. The questionnaire introduction goal 'Group 1' is automatically created for the author.

- DBD_0penFile. This function displays the standard Windows 'open' file dialog box similar to that shown in figure 4.2. The author can choose the questionnaire file they wish to load.

- DBD_SaveFileAs. This function displays the standard Windows 'save as' file dialog box. From this the author can choose the name of an already existing file 
or enter a new filename. The current questionnaire is then saved to a file with this filename.

- DBD_SaveFile. This function saves the current questionnaire under the name that was used to load it. If the current questionnaire has not been saved before then, the DBD_SaveFileAs function is called.

- DBD_Closefile. This function can not be invoked from the file menu. However it is called before a new questionnaire is loaded or created. Its task is to save the current questionnaire and remove it from the internal storage. If the questionnaire has not been saved recently then it will prompt the author to do so. If there is no questionnaire currently loaded then this function does nothing.

Saving a questionnaire is simply the reverse operation of loading it. All the objects within the internal databases are copied back to the external database chains in a new external database. When this has been done the fileinfo chain is updated with the information about the objects in each chain.

When a questionnaire is created or opened, all the information in the external database is copied into equivalent internal databases. Reading the information from the internal databases is very similar to how the QuestEX database manager works. There are a set of five functions which perform these tasks:

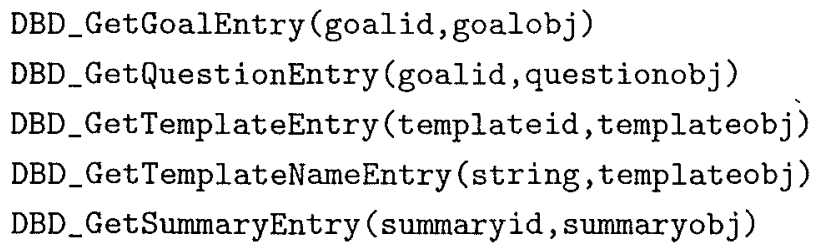

There is an extra template function as templates can bc identified by name as well by the template identifier.

The task of modifying the questionnaire information is suitably straight forward, however it involves a large group of functions. These functions can be divided into three groups: Creating objects, modifying existing objects and deleting objects. These functions are all that is required to manipulate the questionnaire information. The creation functions are:

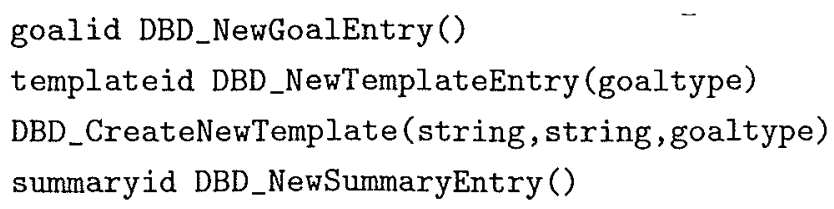

The DBD_NewGoalEntry() function creates both the goal object and the question object. The DBD_CreateNewTemplate function is used to derive a new template from an existing template, see section 5.4.2 for further details. The modification functions are: 


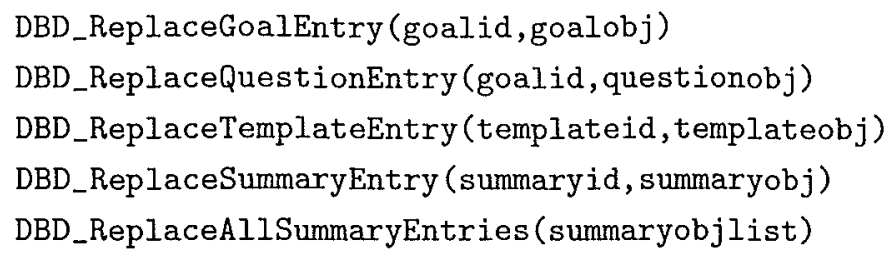

The Summaries in a questionnaire are edited in the summary editor. The summary editor makes temporary copies of all the summaries, so when the summary editor finishes all the summaries are replaced with the last function. The deletion objects are:

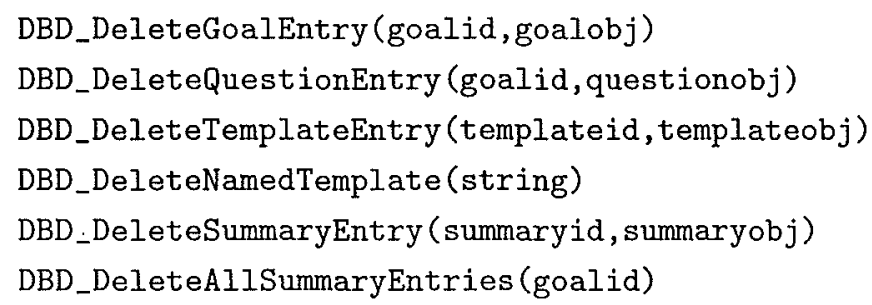

There are two delete template functions as templates can be identified by either name or template identifier. The final function deletes each individual summary entry for a particular goal goalid from each summary. This is used in the DeleteGoal questionnaire operation which is discussed in section 5.3.3.

\subsection{QUESTIONNAIRE EDITOR}

The Questionnaire Editor implementation is based around two concepts:

- Data accessibility. All the questionnaire information that defines a questionnaire must be easily accessible.

- Context sensitive data and control validation. The user can only invoke functions that are sensible in the current context.

The following sections introduce a number of implementations which address these two concepts.

\subsubsection{Tree View}

The Tree View of QuestED represents a questionnaire as a hierarchical tree structure. An example of this is shown in Figure 5.1. The VPI tree package is used to draw the tree. The tree package provides the following useful features:

- A recursive list based tree representation.

- Automatic scrolling and tree window re-sizing. 
- Each node can drawn with a different pen colour and style.

- Each node can be selected with the mouse, and the associated mouse event can be caught and processed.

- The tree font, size and orientation can all be easily changed to suit the author.

- Tree nodes can be collapsed and expanded to hide or show nodes within the tree structure.

These features made the tree package an ideal way for representing the tree based questionnaire data. The tree package represents the tree in a recursive list structure which can only be built in a recursive fashion. This recursive nature can be seen in the following domain declaration which describes each node the tree structure,

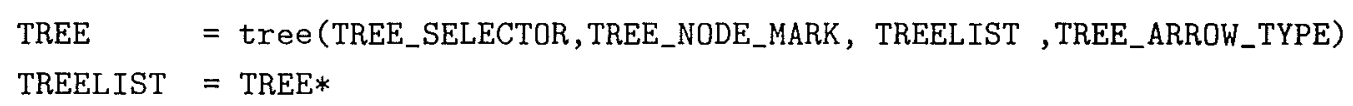

The TREE_SELECTOR field contains the string label of the node, TREE_NODE_MARK indicates if the node has been emphasised and TREE_ARROW_TYPE is a structure which describes how the node is drawn. The TREELIST field contains another list of tree node structures each tree of which is a child node. The tree is built using a recursive routine similar to the QuestEX inference engine in structure. It is a depth first search which maintains an open list to store the goals yet to visit.

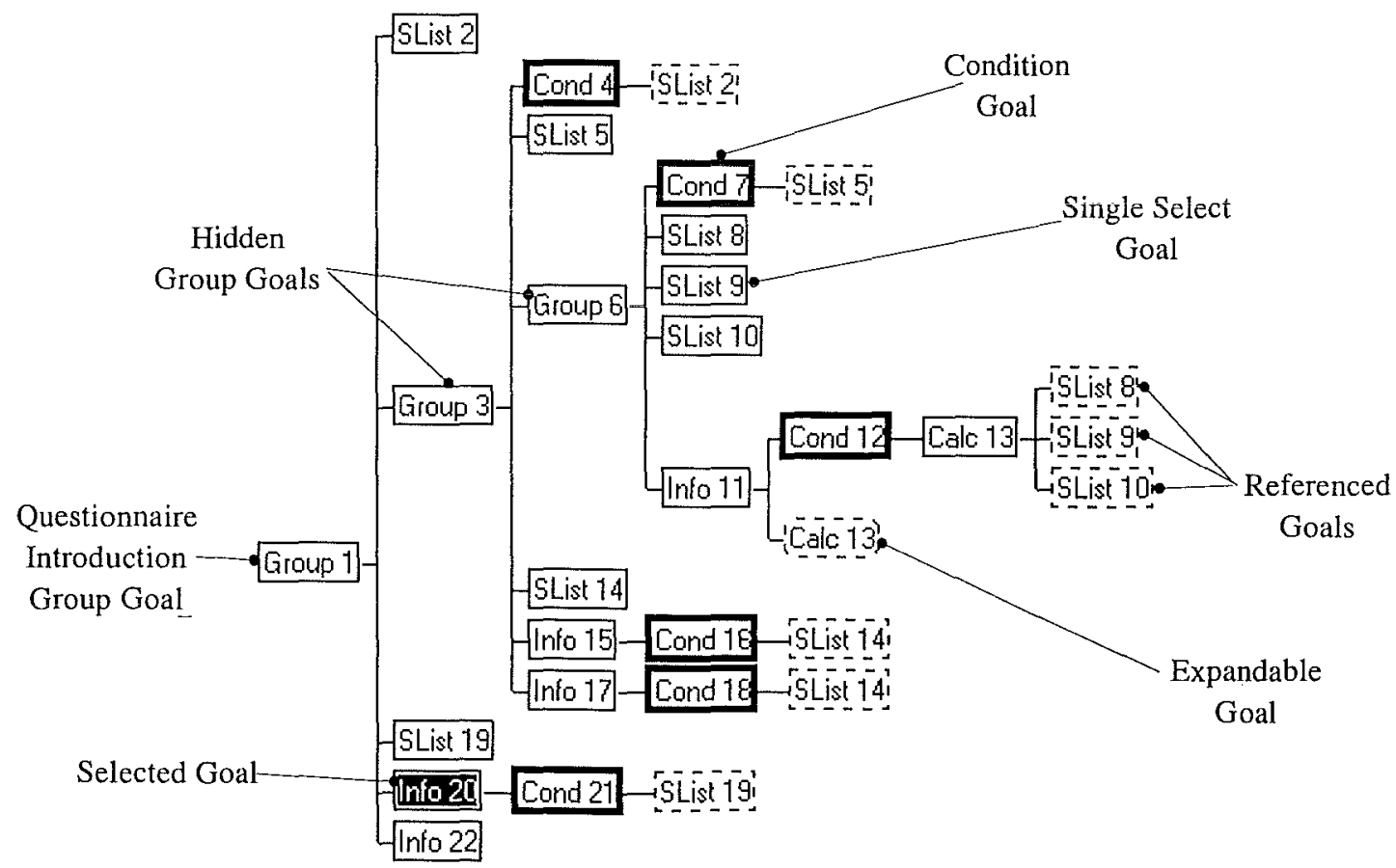

Figure 5.1 The Tree View showing the important features.

Figure 5.1 shows several of the cases the tree scanning routine handles, these are: 
- 'Group 1'. The Questionnaire Introduction goal is always the first goal in the questionnaire tree. It is the only goal in the open list when the depth-first tree scanning routine begins.

- Condition Goal. The Condition goal of a parent goal is always the first in the list of child goal tree nodes. It is drawn with a 'marked' border which is thicker than the borders of the other goals.

- Referenced Goals. A record is keep of each goal processed by the tree scan routine. An internal database is used for this purpose. If a goal has already been encountered then it must be a reference goal. Reference goals are drawn using a different TREE_ARROW_TYPE to differentiate them from normal goals.

- Selected Goal. The currently selected goal appears inverted in the tree view. The mouse is used to select a goal node by clicking on it. When a reference goal is selected, the first actual occurrence of the goal is selected

- Expandable Goals. These goals are parent goals with at least one child, otherwise called control goals. Initially only reference goals are expandable as their child goals have already been displayed further up the tree. Attempting to expand a reference goal will select the first occurrence of that goal. Any control goal can be expanded or collapsed by double clicking on it. This is useful when the questionnaire is getting large and you do not want to see the whole questionnaire while editing.

- Goal Labels. The goals are labelled with a goal type identifier and a number. The numbers generally reflect the order in which they are encountered in a depth-first fashion. Table 5.1 lists the goal labels for each goal type.

Table 5.1 The goal labels of the QuestED Tree view.

\begin{tabular}{|c|c||c|c|}
\hline Goal type & Label & Goal type & Label \\
\hline gt_group & Group & gt_info & Info \\
gt_calc & Calc & gt_condcalc & Cond \\
gt_textentry & Text & gt_numentry & Num \\
gt_singlesimple & SList & gt_multisimple & MList \\
gt_notype & None & & \\
\hline
\end{tabular}

The Tree View can be changed between two modes of operation. These are the horizontal and vertical tree orientation modes. This is a feature provided by the Visual Prolog tree package. The horizontal tree orientation displays the tree with all the sibling child goals arranged on the same horizontal level. The questionnaire introduction goal appears at the top of the tree view. The vertical tree orientation displays sibling child goals arranged vertically and with the root goal at the left hand side. Figure 5.1 shows the tree view in the vertical orientation. Figure 5.2 shows the tree view in the horizontal 
orientation and figure 5.3 shows the Questionnaire Editor Options dialog box which is used to change the view orientation.

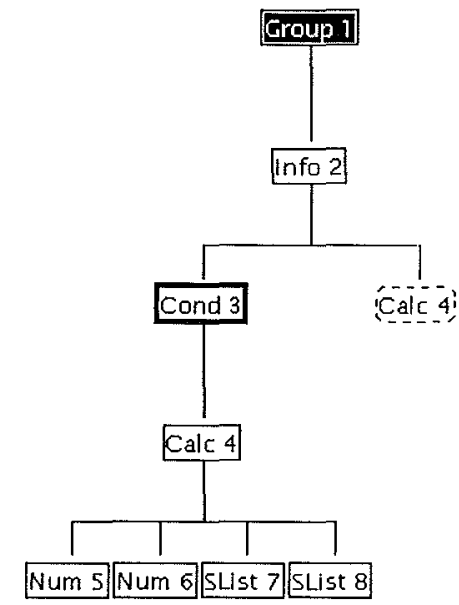

Figure 5.2 The horizontal tree orientation.

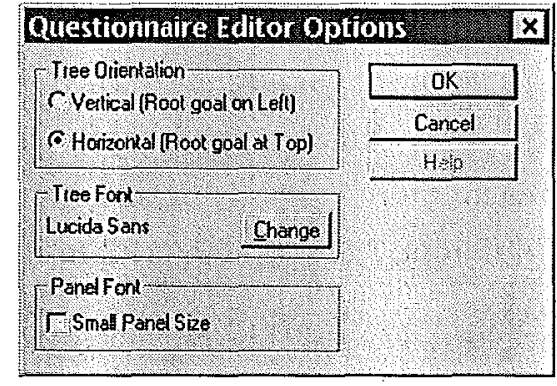

Figure 5.3 The Questionnaire Editor Options dialog box.

\subsubsection{Property Panels}

The property panels provide information about the goals in the Tree view. When a goal is selected in the tree view, the properties of that goal are displayed in the property panel. The goal's properties can then be edited or changed. When another goal is selected, the properties are verified and saved.

Each property panel is a modeless child dialog box. Being a modeless dialog box, it does not have to be closed before continuing with other operations. This is in contrast to a modal dialog box, which must be closed before continuing. For example, an Open file dialog box is modal dialog box, while a floating tool box is a modeless dialog box. As a child dialog box, each panel sits directly on top the main QuestED window. It is a sibling to the tree view window and whenever the parent is moved or re-sized the property panel is also moved or re-sized.

For each goal type, there are two property panel dialog resources. Both are identical except for the dialog box font. The fonts used are 'MS Sans Serif' 8 point, and 'Small Fonts' 7 point. The 'MS Sans Serif' font is the default Windows 95 dialog box font. The 'Small Fonts' font is roughly $80 \%$ the size of the default font. The smaller size enables the largest property panel to fit vertically within the QuestED window at the 640x480 screen resolution. By default the larger panel size is used. The Questionnaire Editor Options dialog box has an option for enabling the smaller panel size. (see figure $5.3)$.

When a questionnaire is loaded into QuestED, there must be a selected goal in the tree view. So the associated property panel must also be displayed. The function 
which manages this process is DUI_ChangePanel. The DUI_ChangePanel performs the following tasks:

- Verifies the information contained in the property panel for the current goal. If the information is not valid, the function returns an error code. Most of the information in each property panel is normally valid. For example the title text, question text and various options need no verification. However the calculation and condition expressions must be parsed, and the result of the parsing process indicates whether the expression is invalid. DUI_ChangePanel returns an appropriate status code to indicate the invalid information in the panel. The author will then be prompted to correct the panel entry.

- Retrieves the validated information for the currently selected goal from the panel as goal and question objects.

- Attempts to load the new dialog panel resource. A check is made if the new dialog panel is the same as the old dialog panel. This occurs if the goal type of the selected goal matches the goal type of the previously selected goal type. The new dialog panel does not need to be loaded. Otherwise the dialog panel is loaded and the QuestED menu is changed to reflect the functions available on the dialog panel. Initially the dialog panel is invisible and has not been positioned within the QuestED window.

- Places the new goal and question object information into the newly loaded dialog panel.

- The size of the dialog panel is calculated, and the dialog panel is moved to the new position and made visible. At this point the dialog panel is drawn in the new window with all the fields containing the new goal information.

- The old dialog panel is destroyed freeing the GDI resources it was using.

Each Property panel contains a number of fields and controls for manipulating the goal information. These are arranged into the following four groups: Goal Information, Reply Field, Options and User Interface. Not all of the property panels contains these groups and the controls and fields within each group changes from goal type to goal type. The advantage of these groups is that the author can associate the same information with a certain group between different goal types. The purpose of each group is as follows:

- Goal Information. This group contains information directly related to the goal as a question in a questionnaire. For example, it may contain the goal identifier, goal type, question text, title text, condition goal and sub goal list. Only the control goals include a sub goal list. 
- Reply Field. Only the question goals have a Reply Field group. In the case of a text based question it contains single line and scrolling options. For the list based questions it contains the list of pre-defined responses and the controls for manipulating the responses.

- Options. These are general options for enabling or disabling many of the user interface functions. This group appears only for these goals that have a user interface. This group may contain options to enable the Restart, Back and Help buttons, and set up help timer and numeric limits options.

- User Interface. Only the goals that can display a user interface have this group. This group contains the functions for setting the goal multimedia options, testing the goal and for editing and selecting a template.

Table 5.2 shows which groups are shown in each panel.

Table 5.2 Caption not done

\begin{tabular}{|c|c|c|c|c|}
\hline $\begin{array}{c}\text { Property Panel } \\
\text { Goal Type }\end{array}$ & $\begin{array}{c}\text { Goal } \\
\text { Information }\end{array}$ & $\begin{array}{c}\text { Reply } \\
\text { Field }\end{array}$ & Options & $\begin{array}{c}\text { User } \\
\text { Interface }\end{array}$ \\
\hline gt_notype & Yes & No & No & No \\
gt_group & Yes & No & Yes & Yes \\
gt_info & Yes & No & Yes & Yes \\
gt_calc & Yes & No & No & No \\
gt_condcalc & Yes & No & No & No \\
gt_textentry & Yes & Yes & Yes & Yes \\
gt_numentry & Yes & Yes & Yes & Yes \\
gt_singlesimple & Yes & Yes & Yes & Yes \\
gt_multisimple & Yes & Yes & Yes & Yes \\
\hline
\end{tabular}

Figure 5.4 shows two examples of property panels, namely the calculation property panel and the text entry property panel.

Dividing the property panels into such groups provides the author with a consistent interface for all the goal types. If the author knows a certain goal should have child goals, then they will be defined in the Goal Information property group.

Although each property panel is a separate dialog box, they are all served by a single dialog box procedure DUI_panel_eh. The use of a single dialog box procedure means this dialog procedure is large, however it takes advantage of many of the common functions the property panels share. A separate dialog box procedure for each of the property panels would lead to large amounts of repetition. DUI_panel_eh serves as a user interface layer between the author and the questionnaire module.

There are two types of changes that can be made to a goal when it is selected the tree view. These are: 


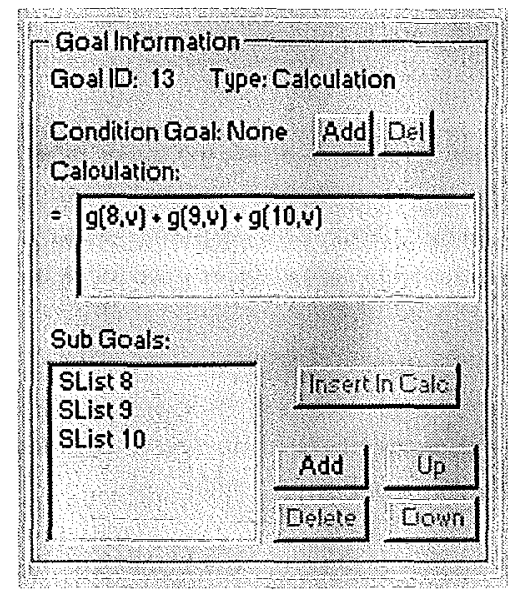

(a) The calculation property panel.

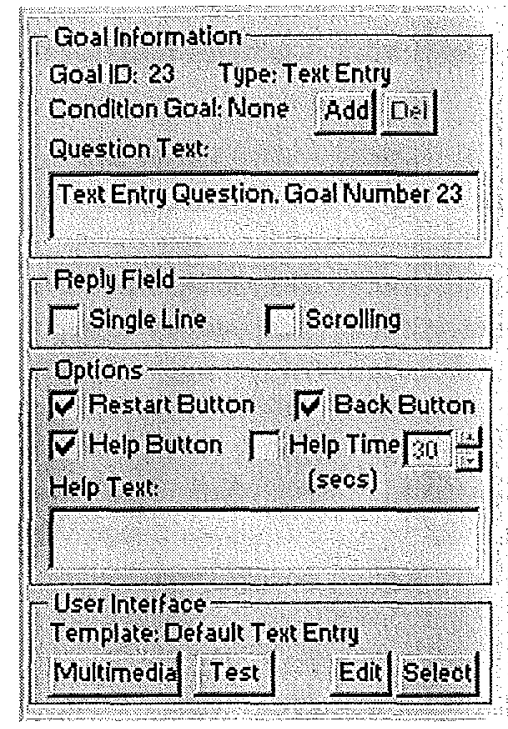

(b) The text entry property panel.

Figure 5.1 Two property panel examples.

1. Panel Changes. This relates directly to the information displayed in the panel. This information can be edited on the panel and is only saved when the questionnaire is saved or another goal is selected. The question text and calculation expressions are examples of panel changes.

2. Questionnaire Operations. This relates to information that maybe displayed on the panel, but can not be edited on the panel. To make a change to this information, an operation must be invoked. This is normally done with a push button control. Consequently a dialog box may be displayed which allows the information to be changed. Alternatively the operation may invoke a simple function which requires no further author interaction. Adding a condition goal or selecting a user interface template are examples of questionnaire operations.

On receiving a user interface event from a particular control, the appropriate DUI_panel_eh clause gathers the relevant information to pass to the questionnaire module. The questionnaire module then performs on operation on the goal data and returns any changes back to the user interface to display to the author.

Not all the controls on each property panel are enabled, and so can not be activated unless certain conditions are met. This is control validation or context sensitive control enabling. This is used through out QuestED, not just within the property panels. It provides visual feedback to the questionnaire author about what commands are available. From this the author can see how the different questionnaire options and functions affect other parts of the questionnaire. Table 5.3 outlines some of the situa- 
tions where the context sensitive control enabling is used. The mode selector controls are the panel controls which state the state or mode of the affected controls.

Table 5.3 Context Sensitive Property Panel Controls

\begin{tabular}{|c|c|c|}
\hline $\begin{array}{l}\text { Mode selector } \\
\text { Controls }\end{array}$ & Affected controls & Purpose \\
\hline $\begin{array}{l}\text { Condition Goal } \\
\text { Add button }\end{array}$ & $\begin{array}{l}\text { Condition Goal } \\
\text { Del button }\end{array}$ & $\begin{array}{l}\text { Initially a goal will have no condition goal, so } \\
\text { the only valid operation is to add a condition } \\
\text { goal. Once added, the opposite situation } \\
\text { occurs since the condition goal can be deleted. }\end{array}$ \\
\hline $\begin{array}{l}\text { Sub Goal } \\
\text { listbox }\end{array}$ & $\begin{array}{l}\text { Sub Goal Up, Down, } \\
\text { Delete and various } \\
\text { Insert buttons }\end{array}$ & $\begin{array}{l}\text { Once a sub goal has been created it appears } \\
\text { in the sub goal list. Once it has been selected, } \\
\text { then it can be moved up, down, deleted or be } \\
\text { inserted into the goal field. }\end{array}$ \\
\hline $\begin{array}{l}\text { Help Timer } \\
\text { button }\end{array}$ & Help Timer field & $\begin{array}{l}\text { Once the Help Timer checkbox has been } \\
\text { enabled, the author can select a time period } \\
\text { using the Help Timer up/down field control. }\end{array}$ \\
\hline $\begin{array}{l}\text { Template Select } \\
\text { button }\end{array}$ & $\begin{array}{l}\text { Template Multimedia, } \\
\text { Test and Edit buttons }\end{array}$ & $\begin{array}{l}\text { Once a template has been selected for a goal, } \\
\text { the used interface functions becone available. }\end{array}$ \\
\hline $\begin{array}{l}\text { Limits check } \\
\text { box }\end{array}$ & $\begin{array}{l}\text { Lower and Upper } \\
\text { Limit fields }\end{array}$ & $\begin{array}{l}\text { The numeric entry goal type can have numeric } \\
\text { limits placed on its response. Checking the } \\
\text { Limits checkbox enables the Lower and Upper } \\
\text { Limit fields. }\end{array}$ \\
\hline
\end{tabular}

\subsubsection{Questionnaire operations}

The questionnaire module consists of a series of functions which deal with the modification of the questionnaire structure and question responses. These functions can be divided into four groups: goal operations, condition operations, child operations and response operations. Each of these functions requires only the information to perform its function and then returns only the changes. This reduces the coupling to the other modules and means the functions can be used at any time by any module. Note that while many of these operations return new goal and question objects, this is only for the convenience of the calling module. These new objects have already been committed to memory by the operation.

The two goal operations work directly on a particular goal. The operations are:

- ChangeGoal (goalid, goalobj, questionobj). For the goal indicated by goalid this operation replaces the goal object and question object with the two input arguments goalobj and questionobj.

- DeleteGoal (goalid,goallist). This is a recursive routine which can delete a branch of the questionnaire tree. The goalid argument keeps track of the highest 
goal to be deleted by this operation. The goallist keeps track of the remaining goals in the branch. Again it effectively works as a depth first search to remove all the child goals of the goal goalid. Each goal is deleted as the depth first search comes out of its recursion. This means the child goals are deleted before the parent goals. For each goal, the goal and question objects are deleted from the questionnaire via calls to the database manager. Also any summary entries for the current goal are removed to maintain consistency between the questionnaire and the summary. Not every child goal in a branch is deleted as referenced goals may be defined outside of the branch. The following rules are apply for each child goal in a condemned branch:

1. If the child goal is unique, (i.e., the child goal is not referenced by another goal) then it is deleted. Consider figure 5.5(a). If goal I1 is deleted, then this rule applies for it and the two child goals.

2. If the parent goal goalid is being deleted and there are all the instances of a particular child goal down the condemned branch, then all these instances of that child goal are deleted. In figure $5.5(\mathrm{~b})$ it is goal $\mathrm{I} 1$ is again being deleted. This rule will apply for both instances of goal Q3 and rule 1 will apply for goals I1 and C2.

3. If there is more than one instance of a particular child goal, and only some of the instances are down the condemned branch, then only these instances of the child goal are deleted. This situation is show in figure 5.5(c). If goal I1 is deleted, then this rule will apply for the first instance of goal Q3, as the second instance of goal Q3 is not a child of goal I1.

The two condition operations manipulate the condition goal of a particular goal, namely:

- AddCondition (goalid,goalobj). A new condition goal is created and the goalid of this new goal is set as the condition goal for the parent goal goalid. The new goal object for goalid is returned.

- DelCondition (goalid,goalobj). The condition goal for the goal goalid is deleted using the DeleteGoal operation. goalid's condition goal is set to 0, i.e. no condition goal.

The five child goal operations work with the child goal list for a particular goal:

- AddChild (goalid, Flag, goaltype, Refgoalstr, goalobj). This operation governs the addition of new goals to the questionnaire. Each new goal is always added as a child of a parent goal goalid. There are two clauses for this operation, each for a different situation. 


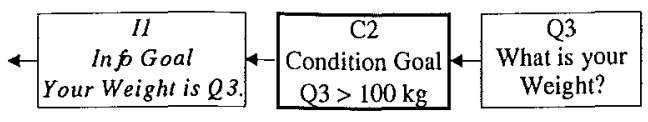

(a) Unique child goal

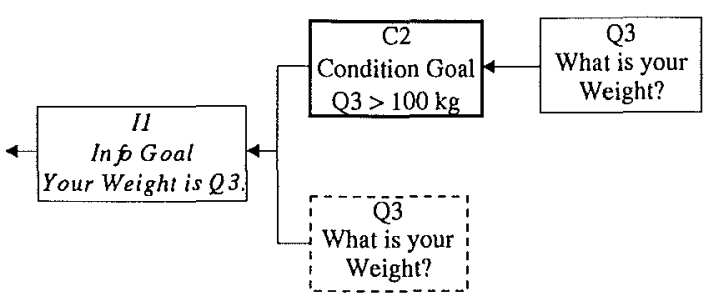

(b) All child goals down the same branch

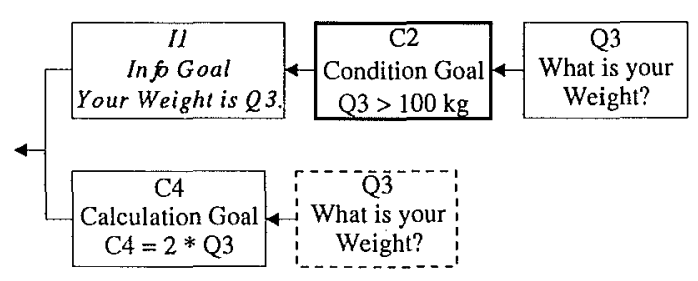

(c) Some child goals down the same branch

Figure 5.5 Examples of the DeleteGoal rules

1. Flag $=$ idc_new_goal and goaltype $=$ valid goal type. A new goal of type goal type is created.

2. Flag $=$ idc_existing_goal. This creates a reference to an existing goal. The refgoalstr argument contains the label of the referenced goal, for example 'Info 1' for information goal I1. This string label is decoded to give the referenced goal id. This goal id is then used to create a child reference goal of the parent goal goalid.

- DelChild(Parentid,Childid,goalobj). This deletes the goal Childid of the parent goal Parentid. The child goalid is removed from the parent's child goal list, and the child goal is deleted using the questionnaire operation DeleteGoal.

- MoveChildUp (goalid,Index,goalobj). This operation is used to modify the order of goalid's child goal list. The child goal at position Index in the parent's child goal list is moved up one position. This is assuming that the goal is not already at the top of the list.

- MoveChildDown(goalid, Index, goalobj). The child goal at position Index in the parent's child goal list is moved down one position. This is assuming that the goal is not already at the bottom of the list. 
The final four operations manipulate the list based response entries for the single select and multiple select goal types:

- AddListItem(goalid, ItemStr, ItemVal, responselist). This operation adds a new entry to the response list of goal goalid. This new entry consists of the response item $r([$ ItemStr], [ItemVal] , [Index $]$ ), where Index is an integer position of the item with in the list.

- EditlistItem(goalid, Index, ItemStr, ItemVal, responselist). This operation replaces the entry at position Index in the response list of the goal goalid.

- DeletelistItem(goalid, Index,responselist). This operation removes the response item at position Index in the goal goalid's response list.

- CopyResponseToGoals (responselist, goallist). This operation is different as it can effect a number of question objects rather than just one. It is used to copy the predefined list responses of one goal to a list of other goals. For example, a questionnaire has ten multiple choice questions, and each question has the same set of responses. The ten questions can be created, the responses can be entered for the first question. This function can then copy the responses from the first question to the remaining nine questions. The responselist contains the responses to be copied and goallist is a goalid list identifying the target goals.

\subsubsection{Calculations and Conditions}

The Calculation and Condition expressions are stored in two forms by QuestED. These are the text representation and a reverse polish notation (RPN) form. The text form is stored because the author can interpret it and the RPN form is stored as QuestEX can interpret this form easily. The expressions are parsed as part of the property panel verification process. If the expression is successfully parsed then property panel process continues uninterrupted. However if a parsing error occurs then an error message is displayed indicating which part of the expression could not be read. The property panel verification process stops here and the author must correct the expression before - continuing.

The parsing process consists of three parts: bracket checking, the actual parsing and child testing. The bracket checking determines if the expression contains the correct number of brackets. The number of left-hand brackets must equal the number of right-hand for the expression to be valid. Once this check has succeeded then the main parsing process begins.

The parsing process is based around the algorithm shown in figure 5.6 (Ralson, 1971, pp154-194). 
Figure 5.6 The calculation and condition parsing algorithm

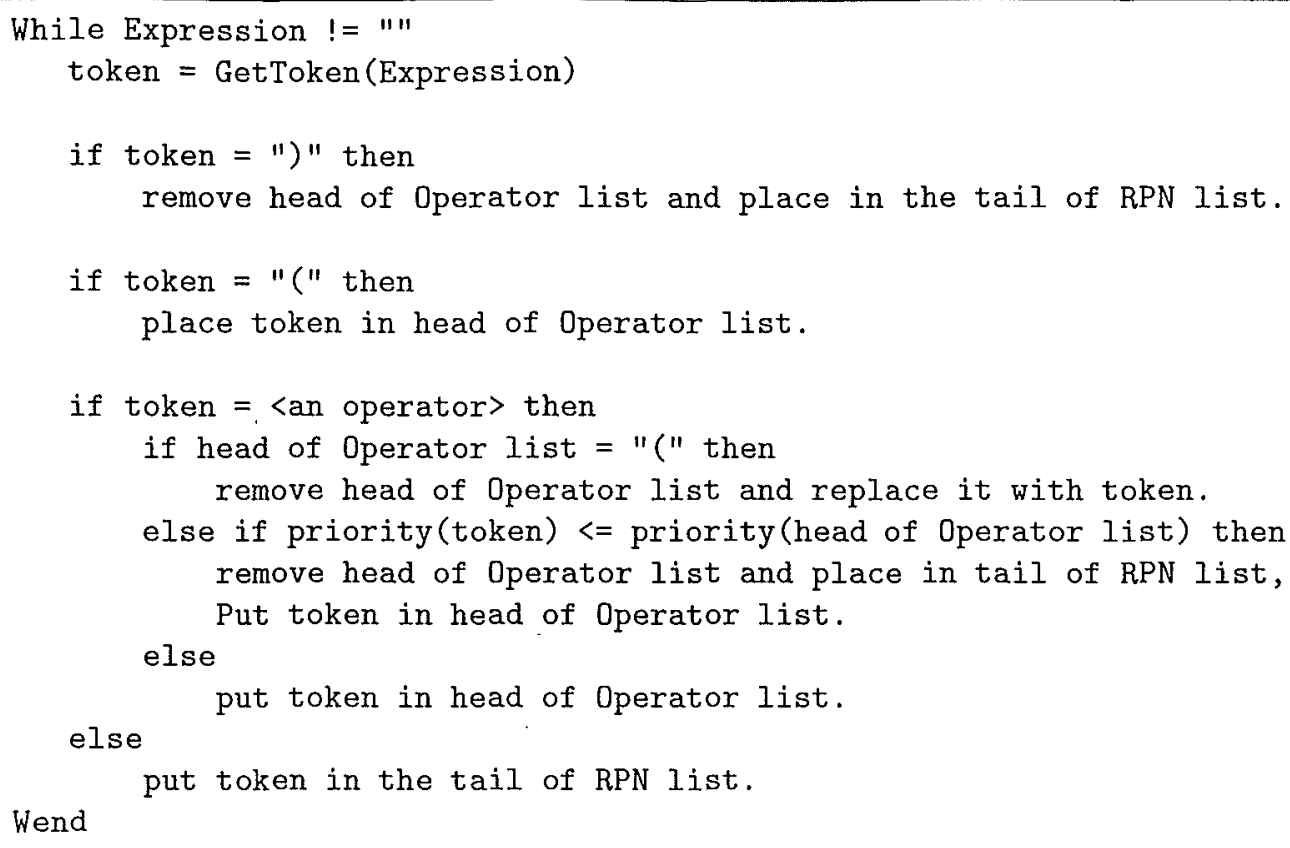

When this process finishes and assuming that the Expression string was well formed, the RPN list will contain the reverse polish notation form of the original expression. The Operator list is used as a temporary stack during the processing of the expression. Table 4.3 lists the operators which the QuestEX RPN evaluator can handle. Each token in the RPN list is then converted into a responselist object as described in section 4.4 .

Child testing is performed to see whether the goal references made within the expression match up with the entries in the goal's child goal list. If a referenced goal in the expression in not a child then a error message is displayed to the author. Only child goals of the calculation or condition goal are allowed to appear in the expression.

A condition consists of three parts: the left hand side, the relational operator and the right hand side. Each of these expressions are converted to RPN form separately. The final RPN form is found by concatenating the left hand side form with the right hand side form and then the relational operator form is attached to the end. This ensures that the relation operator is processed last giving a boolean result.

\subsection{TEMPLATE EDITOR}

Templates are described in section 4.5. Their role is to provide a description of the screen's appearance for each question. The template editor provides the means for editing existing templates and creating new templates.

The template editor consists of two parts, the template manager dialog box and 
the template editing view. Each template contains a template object list. This list contains an ordered series of template objects. The task of the template editor is to allow the questionnaire author to manipulate these template objects within a template.

\subsubsection{Template Manager}

The Template Manager is based around the 'View Templates' dialog box (see figure 5.7). There are five template operations available in the View Templates dialog box and a list of all the templates in the current questionnaire. The availability of each operation is dependent on the currently selected template in the template list. The operations are:

1. Select. This operation selects a template for the currently selected questionnaire goal. This is available only if the type of currently selected questionnaire goal matches the type of the template selected in the template list. For example it is available if the current questionnaire is a group goal and the selected template is also a group goal.

2. Edit. The Edit operation loads the currently selected template from the template list into the Template Editor View, see section 5.4.3. This operation is available for all templates, except the template 'None' for obvious reasons. All templates maybe edited, including the default templates.

3. Create. This operation is used to create new customised templates. See section 5.4 .2 for details.

4. Delete. The Delete operation removes the currently selected template from the questionnaire. A prompt is displayed asking for confirmation for the delete action. If 'OK' is selected, then the template is deleted from the questionnaire. This operation is only available for templates created with the 'Create' operation. The default templates can not be deleted, as they provide the base from which to create new templates.

5. Properties. The Property operation displays a dialog box containing the template properties. There are three properties which can be changed:

- Name. This is the name of the template. Changing this name will change the template name in the template list.

- Invalid Entry. This is the message displayed to the respondent when they make a mistake entering a response, for example if the respondent presses the question 'OK' button without entering a text response in a text entry question. The Invalid Entry message is displayed in the template's 'Help Text' rectangle and a beep is played through the computer's speaker. 
- Display Mode. This governs how the template is drawn onto the screen. The Metafile mode takes less memory but more complex templates take longer to draw. The bitmap mode takes more memory, but always takes a constant amount of time to draw on the screen. Note that the time to draw on the screen does not include the time to render the template in memory. If the template contains any image rectangles, then the Display Mode defaults to Bitmap mode. This is because 256 colour bitmap images can not be correctly rendered into a Metafile device context.

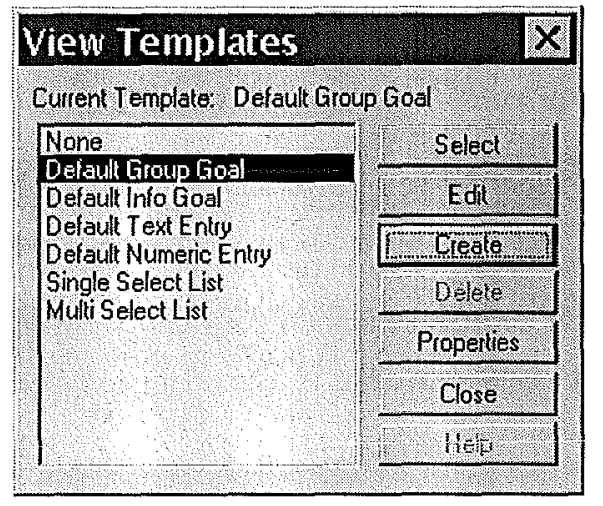

Figure 5.7 The View Template dialog box

\subsubsection{Creating New Templates}

A new template is created by choosing the Create operation from the View Template dialog box. The currently selected template from the Template list is used as the basis for the new template. This base type can be any of the goal types, even one different to that of the intended new template. The New Template dialog then appears, as shown in figure 5.8 .

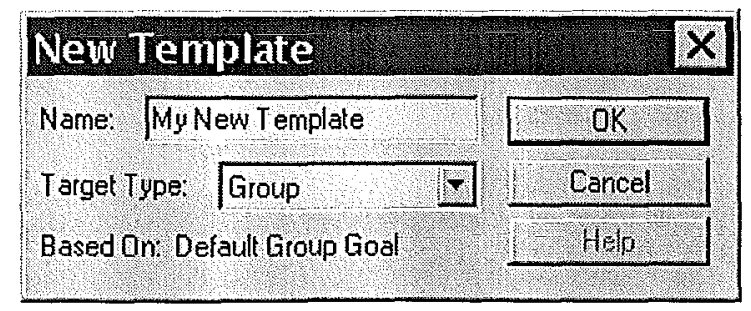

Figure 5.8 The Create New Template dialog box.

This dialog box contains three important fields:

1. Name. This is the name of the new template.

2. Target Type. This drop down list contains an entry for all the goal types that can display a template. The goal types are the group, info, single sel, multi sel, text 
entry and numeric entry goal types. This group of six can be compressed three main template types: information types, list types and the text entry types.

3. Based On. This static text field just indicates which template was selected from the template list in the View Template dialog box.

It is quite legitimate to create one type of template based on a completely different type of template. This allows the author to create a single group goal template with a whole series of customisations and enhancements over the default group goal template. This new group goal template can then be used to create a complete set of templates with this same visual style. The new set of templates inherit the customisations and enhancements from the enhanced group goal.

This is possible because of the structure of the templates. Within each of the three main template types there is only one template object which is unique to that type. For the information types, the unique object is the template title field. For the list entry types, it is the listbox and for the text entry types it is the text field. So by knowing what type of template is being created and what was the basis template, the unique object can be removed and replaced with the appropriate new object. For example, when creating a text entry template from a group goal template, a copy is made of the group goals' template object list. The title text object is removed from the copied template list. The text field object is copied from the default text entry's template object list and is inserted into the copied template object list. So the copied template object contains all the original template objects from the group goal minus the title text object plus the text edit object. Figure 5.9 shows the unique objects in each default template and Table 5.4 lists all the conversion possibilities.
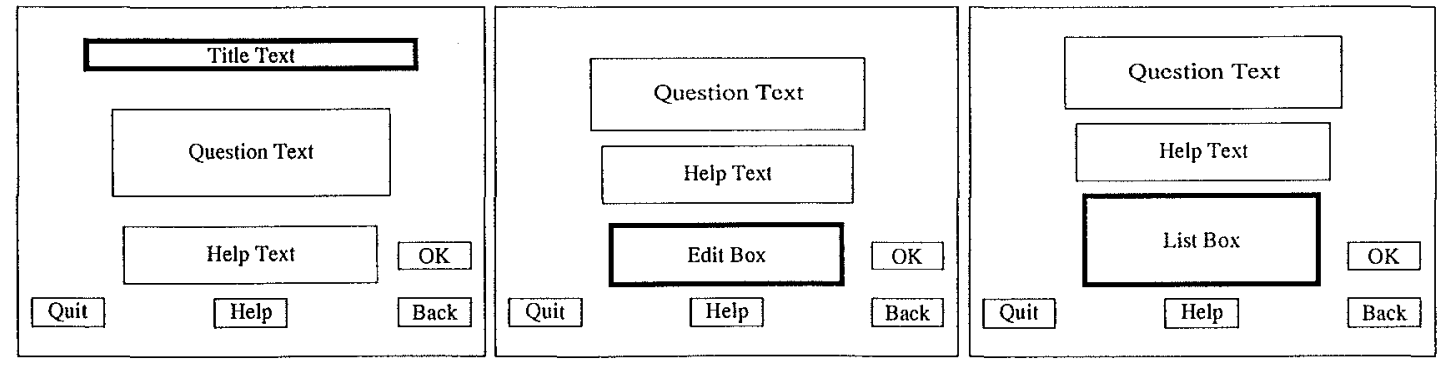

Figure 5.9 The difference between the three types of template is shown by the objects with a dark border.

Table 5.4 The conversion table for the three types of template.

\begin{tabular}{|c|c|c|c|}
\hline From \To & Information types & Text entry types & List types \\
\hline Information types & No change & Add edit, Del title & Add list, Del title \\
Text entry types & Add title, Del edit & No change & Add list, Del edit \\
List types & Add title, Del list & Add list, Del title & No change \\
\hline
\end{tabular}


The whole process for creating a new text entry template from a customised single select template is shown in Figure 5.10. The only problem with this approach is with the positioning of the unique objects in the new template. Since the object is added to the template is from the associated default template, it retains its position from the default template. When this object is inserted into the new template, it will likely overlap some other template object. The author must then manually adjust the position of the object to achieve the correct template appearance.

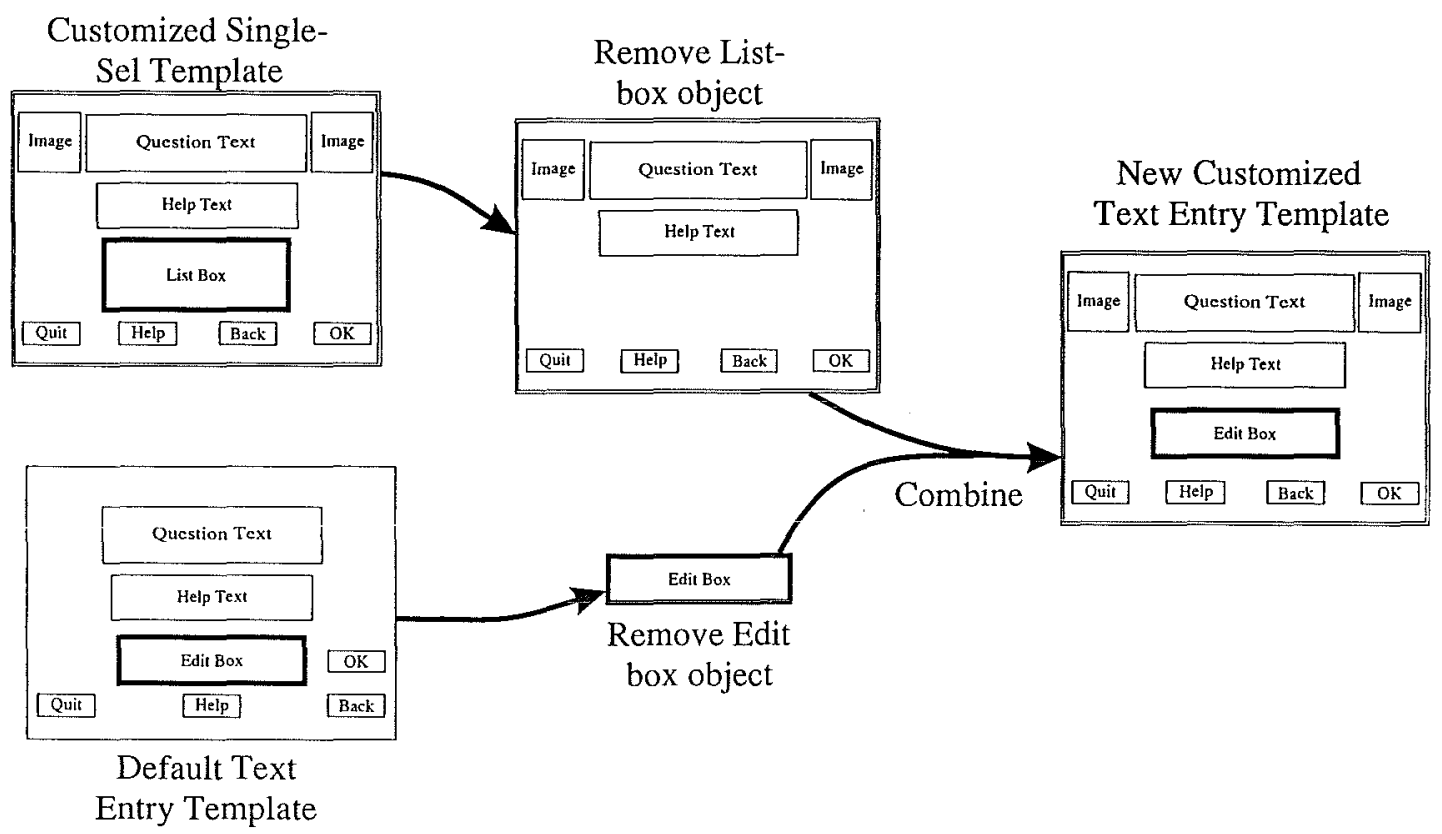

Figure 5.10 The process of creating a Text Entry template from a Single Select List template.

\subsubsection{Template Editor View}

The Template Editor View is shown in Figure 5.11. This view appears in the same frame window that the questionnaire view appeared in. The questionnaire view toolbar and status bar are removed to give a larger view of the template. The floating Template Objects dialog box takes the place of the questionnaire view toolbar. It is shown in Figure 5.12. The Template Objects dialog box contains a list of objects within the displayed template and a series of buttons which perform a series of object operations (see section 5.4.3.3).

\subsubsection{Object View}

The object view appears within Figure 5.11. It consists of the large light grey area just below the menu bar. The object view represents the entire screen display of QuestEX. The template objects which appear within each template are described in Table 5.5. The mandatory column indicates whether the object must appear in every template. 


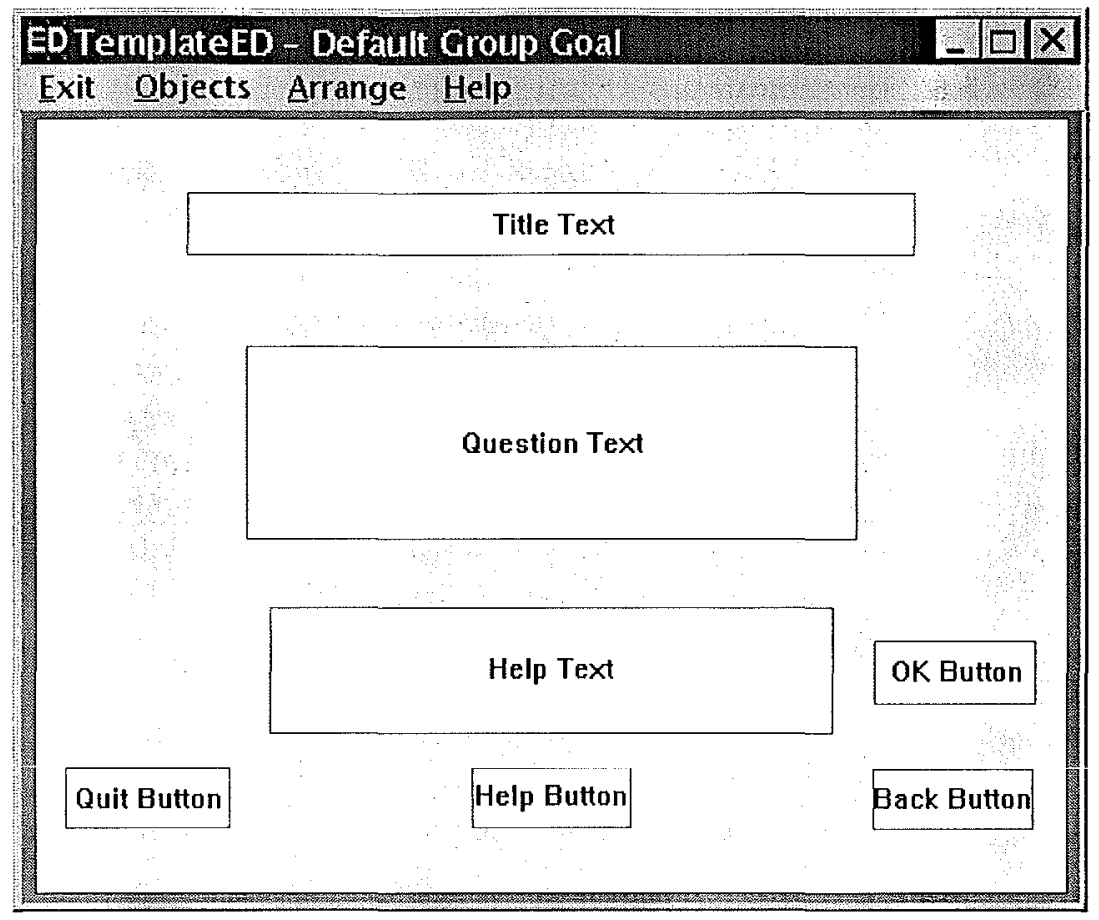

Figure 5.11 The Template Editor View

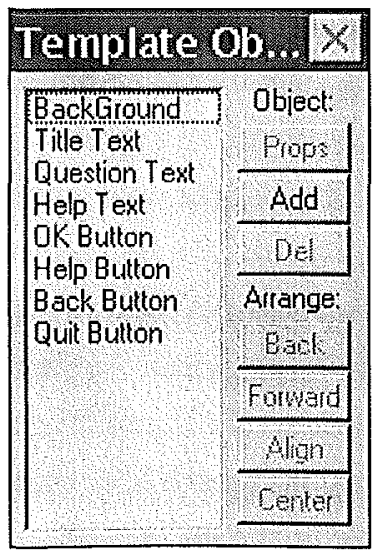

Figure 5.12 The Template Object floating dialog box. 
The three objects with 'Unique' in this column are mandatory for the particular template types that they only appear in. These are the list, text and information templates types.

Table 5.5 The template objects that appear within an object view.

\begin{tabular}{|l|c|l|}
\hline Object & Mandatory & Appearance \\
\hline Background & Yes & Grey area that covers the Object view. \\
Question Text & Yes & White rectangle labelled 'Question Text'. \\
Help Text & Yes & White rectangle labelled 'Help Text'. \\
Title Text & Unique & White rectangle labelled 'Title Text'. \\
Miscellaneous Text & No & White rectangle labelled 'Misc Text'. \\
OK Button & Yes & White rectangle labelled 'OK Button'. \\
Back Button & Yes & White rectangle labelled 'Back Button'. \\
Quit Button & Yes & White rectangle labelled 'Quit Button'. \\
Help Button & Yes & White rectangle labelled 'Help Button'. \\
Edit Field & Unique & White rectangle labelled 'Edit Box'. \\
List Field & Unique & White rectangle labelled 'List Box'. \\
Line & No & Black line labelled 'Line'. \\
Filled Rectangle & No & White rectangle labelled 'Question Text'. \\
Ellipse & No & White ellispe labelled 'Rectangle'. \\
Image Rectangle & No & White rectangle labelled 'Image A', new im- \\
& & ages are labelled alphabetically. \\
Animation Rectangle & No & White rectangle labelled 'Animation'. \\
\hline
\end{tabular}

The object view is drawn with size proportional to the size of the main QuestED window. This has the advantage that the view will always fit within the window, regardless of the window's size. This is beneficial for computers with low screen resolutions such as lap-top computers. This scaling is achieved by using the Windows screen mapping modes to automatically scale the object view graphics. This is achieved in a similar fashion to how QuestEX scales the templates to the screen. Using the VPI library the notation is slightly different. The window mapping mode is set to the arbitrary mapping mode. This is essentially the same as the anisotropic mapping mode used in QuestEX. So the logical coordinate system for the window will stretch to fit the physical coordinate system. The logical coordinate system is set to the constants tmp_xsize, tmp_ysize. These constants are defined as 640 and 480 respectively, this is the size of the lowest screen resolution Windows supports. The physical coordinate system is set to the current size of the window. So irrespective of window size, the logical coordinate system is defined by the constants tmp_xsize, tmp_ysize. Note the main difference between this and the QuestEX is that the physical coordinate system is set relative to the window, not the screen.

The actual template is drawn by recursing through the template object list. Each object in the list is drawn according to the appearance details in Table 5.5. This is a far simpler process than rendering the template in QuestEX because of the simple representation of each object. 


\subsubsection{Object Selection}

There are two methods for selecting template objects within the template view. The first method is to select an object item from the list in the Template Objects dialog box. The object item in the list will then be highlighted. More than one list object can be selected at a time. To select a group of objects, first select a single object by clicking on it, then select a second object while holding down the 'Shift' key. All the objects from the first object to the second object in the list will be selected. Alternatively holding down the 'Ctrl' key will select additional objects individually. This is standard list behaviour in the Windows operating system. A selection rectangle will also appear in the editor view around the associated graphical object.

The second method for selecting is to use the editor view. An object can be selected by clicking on its graphical representation. A selection rectangle will appear around the object. Clicking off a graphical object will select the background object. The corresponding entry in the Template object list is also highlighted. More than one object can be selected by two methods:

1. Select the first object by clicking on it. Then when clicking on additional objects hold down the Ctrl or Shift key. The additional objects will be added to the original selection.

2. Dragging a rectangle around a group of objects. Any objects inside the dragged rectangle are selected.

The selection rectangle is a child window of the main template view window. It is created with no background, no menu, and no caption. This just leaves a sizable border, which is used as the selection rectangle.

\subsubsection{Object Operations}

There are two categories of operations in the template editor, those that can be applied to a single object, and those that can be applied to a number of objects. Most of these operations are available on the Template Editor menu bar and in the series of buttons on the Template Objects dialog box, see Figure 5.12. There is one operation that can be invoked at any time, namely:

- Add. This operation is used to add new objects to the template. Invoking the operation displays the New Template Object dialog box as shown in Figure 5.13. This figure shows the available objects: Misc. Text, Line, Rectangle, Ellipse, Graphic Image and Animation. Only one Animation object can be added to a template, so this item will not appear if it already exists in the template. After selecting the desired object and pressing the 'OK' button, the mouse cursor will 
change to a standard arrow shape and the word 'Add'. By clicking the left mouse button within the template, an object of default size will appear (100 by 60 template units). This new object will be centred around the point of the mouse click. Alternatively the size of the new object can be set by clicking the left mouse button and dragging out a rectangle on the template. When the left mouse button is released, a new object created the same size and position as the dragged out rectangle.

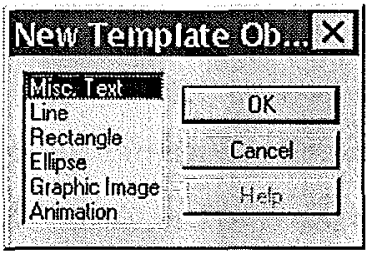

Figure 5.13 The New Template Object dialog box.

The single object operations are:

- Delete Object. This removes the selected object from the templatc. Only objccts that have been added to the template can be deleted. These include the Misc Text, Line, Rectangle, Ellipse, Image and Animation objects. All other objects can not be removed from the template as they perform specific tasks. Some of these permanent objects can be disabled using the property panel user interface options.

- Back. This moves the selected object towards the back of the template. When an object is at the back of a template, then all other template objects will appear in front of the object. An object cannot be moved behind the Background template object for obvious reasons. The Button objects (OK, Help, Back and Quit) and the reply objects (List box and Edit field) cannot be moved back because they are windows which will always appear in front of the graphical objects. The order of the object list in the Template Object dialog box reflects the template order. The object at the back of the template is at the top of the list, whilst the object at the front is at the bottom of the list.

- Forward. This moves the selected object towards the front of the template. The Background object, the Button objects and the reply objects can not be moved forward for the same reasons as in the Back operation.

- Props. This operation displays the object properties. See section 5.4.3.4. This operation can also be invoked by double clicking on a selected object.

- Move. The Move operation is used to move objects around within the template. It is not invoked from the menu or the Template Object dialog box. There are two ways to move an object: 
1. Using the mouse. By clicking the selected object with the left mouse button and dragging, the selection window will move will the mouse. Moving the selection window to a new position and releasing the mouse button, the selected object will be moved to the new position.

2. Using the keyboard. Once an object is selected, it can be moved by using the arrow keys on the keyboard. Pressing one of these keys will move the object 1 unit in the desired direction. Holding down the Shift key while pressing one of the arrow keys will move the object 8 units in the desired direction.

- Centre. This operation displays a prompt dialog which asks the user to select either a horizontal or vertical direction or both. Once chosen the selected object is moved to the centre of the template in the desired direction.

The multiple object operations are only available if more than one object has been selected. The operations are:

- Align. The Align objects operation allows the author align any number of objects horizontally and vertically. The Template Align Objects dialog box is displayed, see Figure 5.14. The dialog box shows the following options. Both the horizontal and vertical options are similar so only the horizontal options are discussed.

- No Change. No change is made to the alignment of the selected objects. They remain in the same position in the selected direction.

- Left Sides/Tops. All the selected objects are moved so that the left side/ top of each object is the same as the leftmost/topmost object.

- Centres. The average coordinate in the desired direction of all the selected objects is calculated. All the selected objects are moved so that the centre of each object equals the average center coordinate.

- Right Sides/Bottoms. All the selected objects are moved so that the right side/bottom of each object is the same as the rightmost/bottom-most object.

- Space Equally. The total amount of space between the selected objects is calculated. This is divided by the number of objects minus 1 . The selected objects are moved so that there is this equal amount of space between each object. Regardless of the size of the objects, there will always be the same amount of space between them. This also works for a negative amount of space between the objects, for example if the objects overlap.

- Centre. This operation displays a prompt dialog which asks the user to select either a horizontal or vertical direction or both. Once chosen the selected objects 


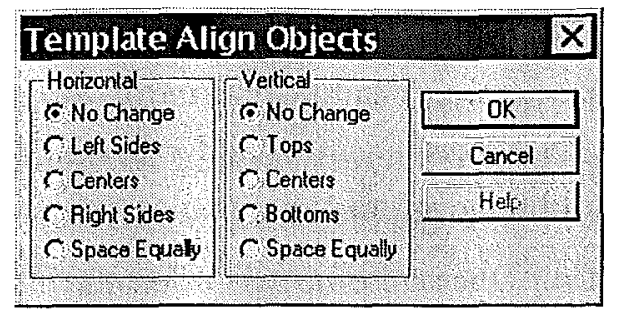

Figure 5.14 The Template Align objects dialog box.

are treated as a single object. They are moved to the center of the template in the desired direction. The relative positioning of the objects is maintained. For example, all four buttons are spaced equally in a horizontal direction. By selecting them all and invoking the center operation, all four can be centred in the middle of the template whilst retaining their relative positions.

- Move. As for the single object case, a group of selected objects can be moved with the mouse or the keyboard.

\subsubsection{Object Properties}

Each template object has a series of properties these are shown in Table 4.5. Once the properties operation has been invoked for an object, the object's associated Property dialog box appears. Because not every object contains the same properties, the dialog boxes differ. However the object types can be grouped into similar objects. For these similar objects a single dialog box is used. The object groups are:

- Text. The Text properties consists of text colour, font, alignment, size and position. The alignment refers to whether the text is left, right, centred or proportionally justified within the rectangle. The size and position of the text rectangle can be changed directly here. The colour of the text is shown by the colour of the 'Text Colour' button's face. Pressing this button displays the Windows Standard colour selection dialog box. The facename and size of the font is displayed under the text colour. The font size is the height of the font in template coordinates. The 'Misc Text' button is enabled when the object being examined is a Miscellaneous Text object. Pressing this button opens a dialog box with a edit field where the miscellaneous text can be entered.

- Graphic. The Graphic properties consists of fill and border colour, size and position. The two colour properties behave the same as in the Text case, as do the size and position properties.

- Button. The Button properties consists of the button text, text and button face colours, button font, size and position. The button text allows the author to 
change the label on a button. The remaining properties behave the same as in the Text case.

- Line. There are two sets of Line properties, line colour and size and position. These both work in the same fashion as in the Text case. There are also two more sets of controls: 'Mirror' and 'Make Line'. Mirror is used to flip the line about either the horizontal or vertical axis. This is necessary because of a limitation of the selection rectangle. It is possible using the selection rectangle to change the size of an object by dragging the bottom right-hand corner of the selection rectangle. However it is not possible to drag this corner above and/or to the left of the top left corner. This is the standard behaviour for a window. This means that the line must always point between 3 and 6 o'clock. By allowing the mirror operations the orientation of the line can be changed to suit all angles. The Make Line Vertical and Horizontal controls set the line either vertical or horizontal. Once again due the behaviour of the selection window it is not possible to set the line either vertical or horizontal by dragging the corner of the selection window. Note also that once a line is either vertical or horizontal it can not be moved or re-sized by dragging the selection window.

- Background. The back ground properties only consist of the background colour. The colour control works in the same fashion as the Text case.

Figure 5.15 shows the property dialog boxes associated with the text, graphic, button and line property groups.

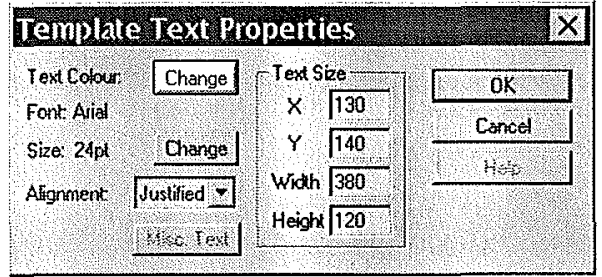

(a) Text dialog box.

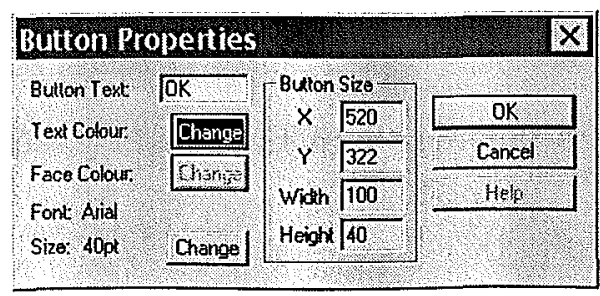

(c) Button dialog box.

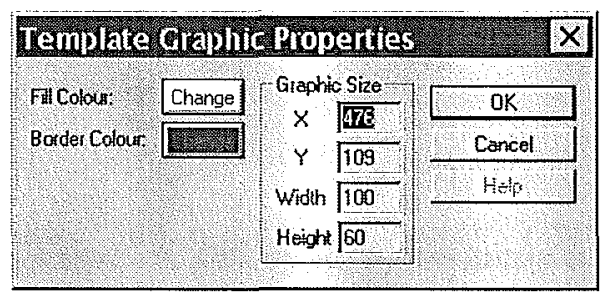

(b) Graphic dialog box.

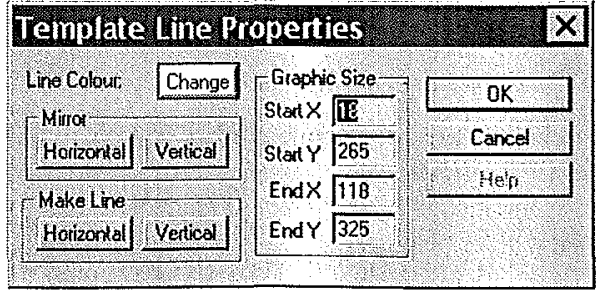

(d) Line dialog box.

Figure 5.15 The property dialog boxes for four types of template object. 


\subsection{SUMMARY EDITOR}

The generation of QuestEX's Summaries are described in section 4.6. Their role is to provide a more comprehendible of form the respondent's replies. The summary editor provides the means for creating customised summaries.

The summary editor is based around the 'View Summaries' dialog box (see figure 5.16 ). This dialog box serves as the summary manager.

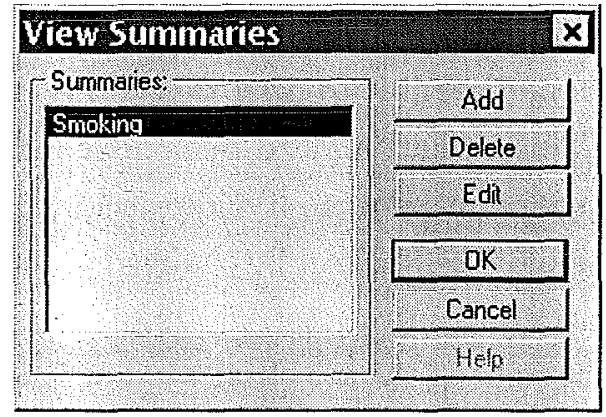

Pigure 5.16 The View Summaries dialog box.

Initially there are no entries in the Summaries list box and the 'Delete' and 'Edit' button are disabled. Pressing the 'Add' button creates a new summary based on the questionnaire as it currently stands. An existing summary can be deleted by selecting it from the Summaries list box can pressing the 'Delete' button. No confirmation is asked for this action, however this action and any other actions within the Summary Editor can be discarded by pressing the 'Cancel' button. An existing summary can be modified by selecting it from the Summaries list box and pressing the 'Edit' button. This will open the Summary Edit dialog box was in discussed in the next section.

\subsubsection{Entering a Summary}

Entering a summary is done in the 'Summary Edit' dialog box. If a new summary is created then the author is first asked if this summary is to be 'Person Readable' or 'Computer Readable'. If they select 'Computer Readable' then the number of options within the Summary Edit dialog box are reduced. This to produce a simple text formatted ASCII file which most spreadsheet and database applications can read. However the method for constructing the summary is no different from the Person Readable summary. Figure 5.17 shows the 'Summary Edit' dialog box.

There are a number of important features in this dialog box, namely:

1. Summary Name. This allows the author to enter a name for their summary. This name only appears in the Summary Editor. 


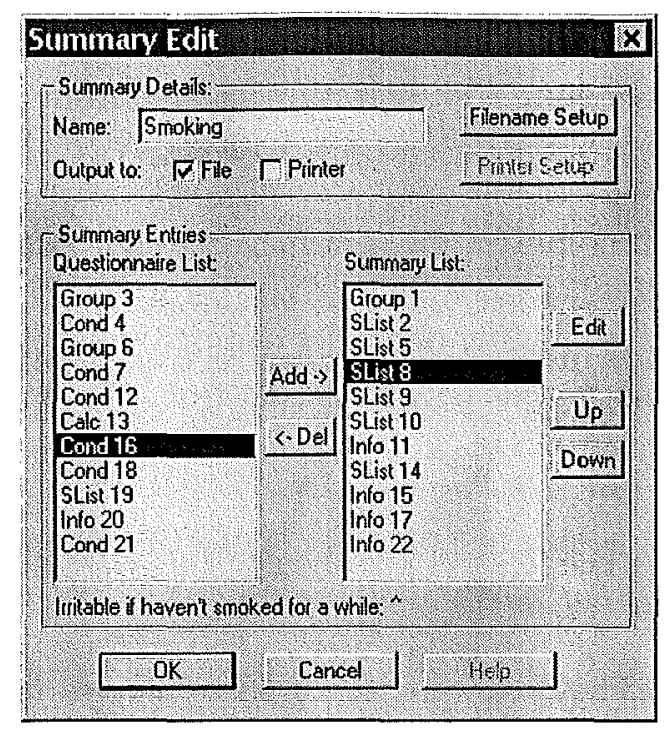

Figure 5.17 The Summary Edit dialog box.

2. Output To. This field is followed by two checkboxes: 'File' and 'Printer'. Checking these options indicate where the summary will be sent after it has been generated. They also enable the following two controls. See section 4.6.1 for further details.

3. Filename Setup. Pressing this button displays the 'Summary File Setup' dialog box. Contained within the dialog are two text fields: Directory and Filename. By default the Directory field is the same as the first eight characters of the Summary Name. The Filename field defaults to 'file'. The information entered here is stored in the sumfile object for the current summary.

4. Printer Setup. Pressing this button displays the 'Summary Printer Setup' dialog box. Contained within the dialog are a printer font field and a 'Stretch to Page' checkbox. The current printer font can be changed by pressing the 'Change' button, this displays the standard Window font selection dialog box. 'Stretch to Page' checkbox determines whether the printer font will be stretched so that the 80 character width summary will fit the full width of the page. The information entered here is stored in the sumprint object for the current summary.

5. Questionnaire List. This list initially contains an entry for every goal in the current questionnaire. It is used to indicate which goals are not included in the current summary. When editing a summary, if any new goals have been added to the questionnaire then they will appear in this list. Multiple entries can be selected in this list. Selecting an item from the list will change the current selection in the questionnaire tree view and the property panel.

6. Summary List. This list contains all the goals which appear in the summary. 
The order of this list is important as it determines the order that each goal will appear in the generated summary. Selecting an item from the list will change the current selection in the questionnaire tree view and the property panel.

7. Add/Del buttons. These buttons transfer the selected items from the Questionnaire list to the Summary list and vice versa. As the Questionnaire list is a multiple select list any number of entries can be transferred to the Summary list. However only one entry can be transferred from the Summary list back to the Questionnaire List.

8. Edit button. Pressing this button will display the 'Summary Entry' dialog box. This is discussed further in the next section.

9. Up/Down button. These buttons are used to change the order of the selected entry in the Summary list.

\subsubsection{Summary Entries}

Each summary is based around an ordered series of summary entries which are introduced in section 4.6.2. When a number of summary entries have been added to the Summary list the author can select an entry and press the 'Edit' button. This will display the 'Summary Entry' dialog box which is shown in Figure 5.18.

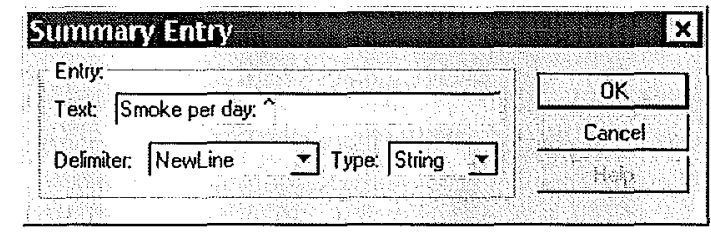

Figure 5.18 The Summary Entry dialog box.

This dialog box allows the author to change the attributes of each entry. By default the text in the Text field for questions, calculations and conditions is the goal prefix string plus goal's associated question text plus the suffix string ': ` '. The goal prefix string is either 'Qstn \%:', 'Calc \%:' or 'Cond \%:' depending on the goal type. The prefix string is attached to give an indication in the summary of the associated goal identifier. The suffix string is necessary as it includes the " ' character which denotes the position where the respondent's reply will be inserted. For group and information goal types the default text is simply their title text. Ultimately the content of the text field is entirely up to the questionnaire author and the default entries are just guides. For computer-readable summaries the default text is simply the " ' character.

The Delimiter field contains a list of seven formatting choices. The particular choice indicates what formatting will be applied to the end of the summary entry text. The choices are: 
- None. Does not apply any formatting. The next summary entry will start immediately after the current entry. This is good for arranging entries into sentences.

- New Line. Forces the next summary entry onto the next line in the summary. This is good for starting new paragraphs.

- Blank Line. Forces the next summary entry to skip a line so that a blank line appears between it and the current entry. Blank line delimiters are good for making section headings.

- Tab. Inserts 8 space characters after the current entry. This will automatically wrap onto the next line if the line length is exceeded. These are useful for tab separated lists and simple columns.

- 1/2 Page Tab. Moves insertion point forward to the next 40 character boundary. This is either in the middle of the page or the start of the next line.

- Centred Line. Centres the current summary entry into the middle of the current line. The next entry appears on the next line. This is good for section headings.

- Right Line. Justifies the end of the current summary entry against the right hand margin. The next entry appears on the next line.

The Type field contains the list of the three standard response types, namely: String, Index and Variable.

\subsection{QUESTVIEW IMPLEMENTATION}

QuestView is the name of the program that implements the questionnaire summary viewing and statistics functions discussed in section 3.2. The intended functions of QuestView are:

1. Viewing of summaries generated by QuestEX.

2. Re-creation of summaries from the raw replies data.

$\overline{3}$. Management of the summary files. This includes the printing, moving, deleting and archiving of summary files which are no longer in immediate use.

4. Management of the raw replies data. This data is automatically saved by QuestEX into an external database after a questionnaire session. The author should be able to export, move, delete or edit the each session replies' within in order to clean any dirty data and improve the quality of information.

5. Generation of statistical information from a set of raw replies. 
Currently QuestView only implements the first item from the above list. The four remaining items need to be implemented before QUEST can be seen as a useful tool.

The finished QuestView will be based around the questionnaire file. Loading this file will identify what summary files and raw replies databases belong to it. QuestView will then offer two operating modes, one focusing the management of the summary text files and the other on the management of the raw replies database. The summary mode will be primarily based around the generation and viewing the summary files. The replies mode will be based around the regeneration of summary files, editing and correcting dirty raw replies and the generation of statistics from groups of replies files.

The statistic function of the replies mode will be concerned mainly generation of simply statistics such as averages, proportions and standard deviations for each goal in the questionnaire. The format of the statistics will vary with each goal type. For instance, Group and Information goal types will only display the proportion of respondents who visited the goal. Numeric entry goal types will be able to additionally display the average and standard deviation for all those respondents who answer the question. The list based goal types will show the proportion of respondents who selected each predefined reply.

\subsection{SUMMARY}

In summary, the QuestED program consists of four main components, these are:

1. Questionnaire database manager. This manages the questionnaire structure information and the loading and saving of this information to a file.

2. Questionnaire Editor. This handles the displaying and editing the of questionnaire structure. The author must use the questionnaire editor when creating a questionnaire.

3. Template Editor. This handles the displaying and editing the of questionnaire user interface. This author's use of the template editor is optional as each questionnaire can use the default user interface.

4. Summary Editor. This handles the displaying and editing the of questionnaire user interface. The author need only use the summary editor when a summarised hard copy of the replies is required, however is it recommended.

QuestED attempts to provide a comprehendible editing interface for creating questionnaires using the information driven model. The editing environment is highly graphical which provides the author with a visual representation of the questionnaire structure and user interface. The next chapter attempts to determine the success of this approach by implementing a series of existing paper questionnaires using the QUEST 
system. These questionnaires and user guide documentation are used in a small trial program. The results of this trial are presented in the next chapter. 



\section{Chapter 6}

\section{PRELIMINARY EXPERIENCE}

In order to determine whether or not the information-driven questionnaire model is successful or not, the Quest system has been distributed to a number of people interested in such a questionnaire tool. Part of this process is converting a number of existing questionnaires to the information driven model. These questionnaires can then serve as examples of what can be done using the system. Also the system has to be documented in the form of user manuals. 'l'he following sections outline a number of converted paper questionnaires, the user guides, feedback from users of the system and a set of recommendations based on the feedback.

\subsection{PAPER QUESTIONNAIRE IMPLEMENTATIONS}

A number of paper questionnaires have been successfully converted to the information driven model. In many of these cases the actual questionnaire has been enhanced through the use of calculations within the questionnaire, interactive user interfaces and information feedback to the questionnaire respondents. A number of these converted questionnaires are described here.

\subsubsection{LifeQuest}

This questionnaire was used as the initial questionnaire for the Quest project, at that stage called LifeQuest. It is a general lifestyle health screen questionnaire. It was envisaged that it would be used in a general practitioner's waiting room. A patient would complete the questionnaire while waiting for their appointment with the doctor. The summarised results of the questionnaire would be immediately available for the doctor who could make an initial assessment of the patient. The LifeQuest questionnaire was hard-coded into the LifeQuest program. While the structure of the questionnaire could be modified to suit the need of the general practitioner, the LifeQuest program could not be adapted to another questionnaire. The content of this questionnaire was derived from the Health Check questionnaire, which is described in section 6.1.6 and a Health 
and Fitness Questionnaire. See appendix C.1 for the paper version of the HealthCheck questionnaire. The origin of the Health and Fitness Questionnaire is unknown.

Initially the LifeQuest questionnaire consisted of five sections, personal, drinking, smoking, diet and safety. However the total number of questions within the questionnaire was limited due to the DOS memory constraints. When implemented in the first Windows version of Quest, the LifeQuest questionnaire included an additional medical section. The number of questions were increased to provide a better mix of questions. However the actual value of the questionnaire was limited as it had not been designed for a particular survey, but was rather a collection of questions from two different questionnaires.

\subsubsection{Stroke Questionnaire}

The Stroke questionnaire is a very simple demonstration questionnaire which shows how to set up a simple calculation. It is based on the calculation of a risk factor which indicates whether a patient is at risk from stroke. The risk factor calculation was devised by Dr Will Coppola and presented in the British Journal of General Practice(Coppola, 1994). See appendix C.2 for more information on this questionnaire. This questionnaire is used in the QuestED tutorial, see section 6.2.

\subsubsection{Smoking Questionnaires}

The smoking questionnaire was used to demonstrate the capabilities of Quest at the Royal New Zealand College of General Practitioners Conference in Christchurch, 1995. Co-Supervisor Dr Keith Carcy-Smith suggested a questionnaire from the "Front Line" smoking cessation programme, "Helping your Patients Stop Smoking". This questionnaire was arranged by D Kent and P Barham in 1986 at the Auckland School of Medicine. The original source was the article "Guidelines of Smoking Cessation" by M Kunze and M Wood (Kunze and Wood, 1984) which appeared in UICC Techincal Report. The purpose of this questionnaire was to demonstrate the ability to ask a series of questions on patient smoking habits. The replies are then used to determine if the patient is addicted to nicotine. The questionnaire is based two groups of three single choice questions. Each question is a five point rating between 'Always' and 'Never'. The sum of the ratings from each group of questions indicates the dependence of the patient on nicotine and their motivation for quitting smoking. The implemented questionnaire has a number of miscellaneous questions which gather patient details and a series of conditional information goals which provide feedback to the patient when certain conditions are met. Appendix C.3 contains a table of the main questions in the smoking questionnaire. This questionnaire is used in the QuestED tutorial, see section 6.2 . 


\subsubsection{Readiness to Change Questionnaire}

The Readiness to Change Questionnaire was developed by the National Drug and Alcohol Research Centre at the University of New South Wales in Australia. It is a twelve question questionnaire which determines the stage of the respondent in overcoming a drug or alcohol problem. There are three possible stages: pre-contemplation, contemplation and action. Pre-contemplation indicates the respondent has not thought about changing their lifestyle. Contemplation means the respondent has started to think about changing their lifestyle, and action means the patient is attempting to change their lifestyle. The twelve questions are divided into three groups of four and are ratings which range from 'Strongly Agree (+2)' to 'Strongly Disagree (-2)'. Each group corresponds to one of the three stages and the sum of the ratings for the questions within the group form the group score. The group with the highest score indicates the stage of the respondent. Appendix C.4 gives the entire questionnaire and marking schedule.

\subsubsection{AUDIT Questionnaire}

The AUDIT questionnaire's purpose is to determine if the respondent has a drinking problem. It uses a series of ten rating single choice questions. Each question's rating revolves around frequency scale which typically ranges between 'Never (0)' to 'Daily or almost daily (4)'. The sum of all the questions is calculated to give an overall score. See appendix C.5 for the complete set of questions. The overall score is interpreted as in Table 6.1.

Table 6.1 The AUDIT questionnaire scoring scale.

\begin{tabular}{|c|l|}
\hline Score & Interpretation \\
\hline$<8$ & No drinking problem. \\
$8-10$ & Hazardous drinking style. \\
$>10$ & Severe drinking style. \\
$<11$ & $90 \%$ chance of not being a problem drinker. \\
$>11$ & $80 \%$ chance of being a problem drinker. \\
$>25$ & Alcohol dependent. \\
\hline
\end{tabular}

\subsubsection{HealthCheck Staff Questionnaire}

In 1994, the Royal New Zealand College of General Practitioners (RNZCGP) Research Unit at the University of Auckland School of Medicine produced a report on computer assisted health screening programmes (McAvoy et al., 1994). The focus of this study was a computer program called HealthCheck. HealthCheck is described within the report as "a computer assisted, self administered lifestyle assessment questionnaire designed to be used independently by patients attending their general practitioner. The 
programme includes questions on modifiable risk factors such as alcohol consumption, cigarette smoking, diet stress, weight, blood pressure, cervical screening, breast examination, immunisation status and use of over the counter medication". This programme was developed in 1988 by Dr John Litt, Senior Lecturer in General Practice, Flinders University Medical School, Australia. The aims of the study were to: 1) assess the merits of HealthCheck for patients and staff at a sample of general practices and 2) assess staff attitudes during this survey. A series of additional questionnaires were created to meet these aims. There was a patient questionnaire, three staff questionnaires and a phone interview questionnaire. The HealthCheck Staff questionnaire implemented here comprises the first two staff questionnaires. See appendix C.6 for the full questionnaire.

\subsubsection{Teenage Health and Fitness Questionnaire}

The origin of this questionnaire is unknown, it is different from the Health and Fitness Questionnaire mentioned in section 6.1.1. The Teenage Health and Fitness Questionnaire is a confidential health screening questionnaire aimed at assessing various aspects of a teenagers lifestyle. The aims of the questionnaire are stated to be to:

- detect any problems that the subject may have and wish to discuss further, either with the doctor or nurse.

- improve the care the doctor and practice nurse can give the subject and young people generally.

The questionnaire covers: general health, relationships, personal safety and a series of general questions. It is almost completely implemented using single selection questions. The majority of these questions are 'Yes/No' questions and the remainder are variations on a frequency scale, i.e. 'Frequently/Occasionally/Never'. See appendix C.7 for the full questionnaire.

\subsection{THE USER GUIDES}

There are three manuals which document the Quest system. These are the QuestED user guide, the QuestEX user guide and the QuestED tutorial. At this stage there is no on-line help built into the system. Eventually a fully context-sensitive help file is planned which will provide a complete description of the system and how it works. Appendix D contains a copy of each of the manuals. The QuestED user guide is divided into four parts. The first part describes the questionnaire concepts behind the questionnaire system and the information driven model. The questionnaire editor part describes the installation of the Quest System, the user interface and the operations used to manipulate the questions and questionnaire structure. The template editor part describes the purpose of templates within Quest, template editor user interface 
and the operations that can be performed on the template and the template objects. The summary editor section describes how the a questionnaire summary is generated and the operations used to generate a summary.

The QuestEX user guide is divided into two parts: the author's user guide and the respondent's user guide. The author's user guide describes how the system is installed and run. This includes loading the appropriate questionnaire file and setting up the default printer. The respondent's user guide provides a set of instructions on how to complete a questionnaire. It outlines the questionnaire introduction, what appears on the screen and how to interact with it. The section introductions and question are outlined in a similar manner.

The QuestED tutorial provides two simple questionnaires which a questionnaire author can use to familiarise themselves with the questionnaire editor. The first is a simplified version of the smoking questionnaire introduced in section 6.1.3. It first analyses the problem of what information is required. It then breaks the problem down in order to identify the questions, the question relationships and the question order. The tutorial then goes through the steps to enter this questionnaire into QuestED. The second questionnaire is the stroke questionnaire which was introduced in section 6.1.2. As the actual questionnaire is quite simple, this tutorial focuses on creating a summary for the questionnaire and modifying the questionnaire user interface.

\subsection{THE TRIALS PROGRAMME}

There have been two trials of the LifeQuest and QUEST systems with prospective general practitioners. The first was instigated as part of a Masters of Engineering Management project by Ropate Siwatibu. Ropate's project was a business plan study of QUEST with the focus of the plan on applying QUEST to medical health screening tasks (Siwatibau, 1994). His trial targeted general practitioners in the Christchurch area and included a paper questionnaire and a follow-up personal interview. A personal interview was offered to eleven general practitioners who were interested in the QUEST system as a tool they could use in their practice. In each interview they were shown QUEST version 1 which implemented the Question Order Flow model. A number of suggestions, comments and problems were made regarding this version. A brief summary of them are:

- Suggestions.

- Must be more user friendly.

- Should implement a full (medical) questionnaire when selling the package.

- Must have sections on screening, family history, lifestyle and past history. 
- Patient information need to be able to be updated, e.g. if a patient changes job.

- Must have a scoring system to provide feedback to patients.

- Get rid of mouse.

- Change default selection to most questions.

- Have a section on sexual safety.

- Give patients the option of skipping sections or not filling in questions.

- Have animated graphics on screen to attract patients.

- Have a central database whereby GPs could compare their data.

- Vital to have risk factors highlighted by the program.

- Comments.

- Vast potential for gathering data.

- Could get nurse to help patient fill out questionnaire and collect other information such as height, blood pressure, etc.

- First time patients should be guided through the first sitting.

- Concern about patients tampering with the computer.

- Need to stress confidentially to the patients.

- Problems.

- Need new hardware.

- Need more paper for print outs.

- Needs to be compatible with other medical database software.

A number of the suggestions related directly to the content of the LifeQuest questionnaire. These are of a greater concern to the questionnaire author and can be largely disregarded in this discussion. However it is important that the questionnaire system can provide all the facilities to accommodate the needs of the questionnaire author. The suggestions relating to the user interface of questionnaire were considered for QUEST version 2. For example attempts were made to improve the user interface and to include graphics and animation to attract the patients. However the mouse was not removed as it is seen as a worthwhile method of replying to questions. The suggestions about highlighting risk factors and the scoring system were provided for by the new calculation and condition goal facilities. This is a general solution to the scoring system problem as it relies on the questionnaire author to create the scoring system within in questionnaire. This approach can be applied and adapted to a wider range of applications than if the scoring system was simply targeted at lifestyle questionnaires. 
The comments again were largely directed towards the content of the questionnaire and the management of how the questionnaire is administered. The comment about requiring a nurse to guide the patient through the questionnaire is a potential problem as this somewhat defeats the point of having the computer administer the questionnaire. It may be necessary to have a nurse walk the patient through the first few questions in order to show the patients the basics of the system. Efforts have been made in QUEST version 2 to make the system more secure against respondents who may wish to tamper with the computer. It is now possible for QuestEX to 'take over' the operating system and stop the respondent from leaving the questionnaire either by task switching away or by rebooting the computer with the 'Ctrl-Alt-Del' key sequence. This however does not stop the respondent turning the computer off and then restarting it.

The problems section reflect the changing personal computer scene. New application invariably seem to need more computing resources than before. The QUEST version 1 was a set of fairly light weight Windows programs in terms of computer resources. QUEST version 2 has followed recent trends and is more resource hungry, and this is largely due to the use of the Visual Prolog libraries in QuestED and the multimedia capabilities of QuestEX. So the requirements for new computer hardware are higher. The problem with summary print-outs have been addressed through the new summary manager. By being able to decide exactly what goes into a summary the author can reduce paper consumption and increase the amount of relevant information on each summary. The compatibility problem with other applications was considered. The best solution was as a simple text exporting capability. Most spreadsheet and database application can import text files. Again this is a general approach to the problem as a solution involving one or two medical database problems would have been too restrictive.

The second trial involved a small group of general practitioners who expressed an interest in an early prototype version of QUEST version 2. This version was demonstrated at the Royal New Zealand College of General Practitioners Conference in Christchurch, 1995. From this a group of eleven from throughout New Zealand received a test version of QUEST version 2 along with the user guides and examples presented in the previous sections. The idea was to get them familiar with the questionnaire model by reading the user guides and by following the tutorial. An evaluation questionnaire was included with the example questionnaires as a means of providing feedback on the new version of QUEST. During the trial period a number of updated versions of QUEST version 2 were sent out to the recipients. These updated versions fixed a number of problems which arose through the testing process. Unfortunately the results of this trial were not as successful as the previous trial. This could be attributed to a number of reasons:

- Bad experiences with one of the early releases. In total there were three updates sent out to the recipients and one letter explaining how to rectify further problem. 
These updates fixed specific problems which only appeared during the testing phase. One of the problems was particularly bad in that it was able to render the Windows operating system unusable. This type of experience may have soured the recipients' view of QUEST.

- Poor choice of test population. The choice of general practitioners as test recipients seemed necessary as they were the targeted users in the case of a Lifestyle questionnaire. However general practitioners are particularly busy people at the best of times, and many of them may not have had the resources and time to investigate the QUEST system a tool useable in their practice.

- The trial group was too small. To produce a meaningful result, the size of the trial group should have been larger. This would have resulted in more general feedback about the system.

- Lack of time and resources. This would have allowed more communication between the recipients and the project author. Extra time would have allowed a more thorough investigation of the general practitioners' problems with the system.

However a number of positive outcomes occurred from the amount of preliminary feedback from a small few of the recipients. The author's own feedback and experiences were also useful in evaluating the problems with the questionnaire model and the subsequent implementation.

The problems seen in the system are most important are they indicate where the system needs adjustment. Some of the problems seen were:

- The terminology used was not obvious. Much of this terminology has come from the knowledge engineering aspects of the project. This would confuse people unfamiliar with this field.

- There needs to be a summary display for the patient. Currently only a print-out is available which is not as effective as a summary displayed on the screen.

- Summary editor was logical but laborious. This process is not stream-lined enough especially when compared to the questionnaire and template editors, both of which are graphical in nature.

- Performance of QuestED is slow for large questionnaires. The questionnaire tree view window slows down markedly once a questionnaire grows large. This is problem with the VPI tree package.

- There is no way of including the replies of one goal in a question goal. The question goal types can not include references to other goals with their question text as the Info goal type can. 
- The LifeQuest questionnaire is not up to scratch. The actual design and content of this questionnaire is some what unsatisfactory for use in a general practice.

- The replies viewing aspects of the system are currently lacking. QuestView is currently only a simple summary viewer which allows no process of the replies. Such processing should include the cleaning of erroneous replies data, management of the raw reply files and summary files, and generation of text summaries and statistics.

The advantages of the system are equally important. Some of the positive feedback about the system was:

- Tree representation was less confusing than the flow chart representation for complex questionnaires.

- The questionnaire model is sufficiently simple to understand when creating questionnaires.

- The graphical display of QuestEX was especially good for young children. Children often see the questionnaire as a fun activity and will complete the questions without realising they are involved in a study. The multimedia capabilities are especially useful here.

- The template editor gives the author a great deal of flexibility in creating their own user interface. This is an important advantage as it gives the patient something familiar to identify with.

\subsection{FUTURE RECOMMENDATIONS}

The last section presented a number of advantages and disadvantages. The disadvantages need to be addressed before the system can be used in either a general practice or in another field. Along with presented disadvantages there are a number of recommendations for each program component of the system. These are:

Questionnaire Model.

- Generalise the model further by allowing question goals to have child goals. The child goals would be used to create the question text in much the same manner as Info goals do now. This would mean that all goal types can have children. 


\section{QuestEX.}

- Include a summary display screen. This would display a summary to the respondent once the questionnaire had finished. At present only a summary print-out is available which does not have the impact of seeing the completed summary appear on the screen soon after finishing the questionnaire.

- Improve the 'back' function. Currently this does not perform consistently. This is mainly due to the way Group goals are processed and must function in an expected manner otherwise the respondent may be confused.

- Add further multimedia enhancement to the system. Possibilities include touch screen or Pen for Windows support and adding text to speech capabilities so the questionnaire can be read out to the respondent. Both of these enhancements will improve the useability of QUEST by hiding the fact that it is a personal computer based system. A speaking, keyboard-less touch screen based implementation will appear less like a computer and so will be more accessible to people not used to dealing with computers.

QuestED.

- Provide a list based view of the questionnaire tree. This would be an alternative method of editing the questionnaire structure. A list based view would be perform inherently faster than the graphical tree view. This is because a list does not have to perform the same amount of calculation to draw the list as the tree does. The list view would still show the questionnaire tree in a hieratical form but in a similar fashion to the directory view in the Windows File Manager.

- Improve the summary editor. Currently the summary editor has been described as 'logical but laborious'. It is clearly not up to same the standard as the questionnaire editor and the template editor. Efforts must be made to produce a more form based approach to editing the summaries. An approach based around the VPI hypertext editor looks the most promising. Hypertext links could be used to represent references to goals within the questionnaire structure. Clicking on the hypertext link would allow the reference to be - edited.

- Produce on-line help for the QUEST system. This would be accessible through QuestED. Currently the only help available is through the user guides. Such a help system would be based around the existing user guides but would bring together the information in the user guides into a single reference.

- Generally improve the user interface of the questionnaire editor. This includes re-organising the menu structure, adding drop and drag functionality 
to the tree view, be able to cut and paste goals and provide context sensitive help and menu handling. These improvements will bring QuestED into line with a number of popular Windows applications. So making easier for questionnaire author to learn the QuestED user interface.

QuestView.

- The functions discussed in section 5.6 need to be implemented.

Once these recommendations have been implemented, QUEST will provide all the necessary features to implement a small to medium scale survey. The intention is that the QUEST system be made available to those people and organisation who have expressed an interest in it. Currently these include Dr Keith Carey-Smith for the Quality Assurance department of the Royal New Zealand College of General Practitioners and more recently John Kaiser at the Centre for Information Management at the University of Otago. Another option is distribute QUEST as a shareware application using the frec advertising opportunities offered by the World Wide Web and the shareware archives such as Simtel and Winsite. 



\section{Chapter 7}

\section{CONCLUSION}

In conclusion, this thesis has examined the process of implementing a questionnaire or a survey from concept to conclusion. The questionnaire process is a sequence of tasks which encompasses the aims, design, implementation, presentation, analysis and conclusion of a questionnaire or survey. It is important to appreciate the process as a questionnaire is not simply a collection of related questions. There is a purpose and method behind each question.

Two questionnaire models were designed for the questionnaire process: the Question Order flow approach and the Required Information flow approach. The models view the questionnaire process differently. The Question Order flow model views each questionnaire as a collection of sequenced questions. The Required Information flow model views the questionnaire as a hierarchical tree of information with the most general information at the root of the tree and the most specific on the leaves.

Both of these models have been implemented. The Question Order flow model was shown to be flawed and the implementation of this model showed this to be the case. This was due to the way that questions and conditions refer to the replies of other questions. The Required Information flow model was designed to overcome these flaws. The implementation of this model successfully demonstrated better reliability in performance and in the representation of a questionnaire. The implementation of the two main components in this system are presented: the questionnaire editor and the questionnaire administrator. The questionnaire editor program allows an author to design a questionnaire and implement its structure, user interface and output. The questionnaire administrator program interprets this information and can present it to a series of questionnaire respondents. The respondent's replies are then summarised and can be further analysed in order to produce a conclusion.

The results of two trials for both the Question Order flow model and the Required Information flow model are presented. The trials together were only marginally successful but did produce a number of useful recommendations and user feedback. This feedback has helped to shape the project and has lead to a number of future recommendations on what should be done to complete the project. 



\section{REFERENCES}

ALLEN, B. and SKINNER, H.A. (1987), 'Life assessment using microcomputer', In BUTCHER, J.N. (Ed.), Computerized Psychological Assessment, Basic Books Inc., Chap. 7, pp. 108-123.

BARKAKATI, N. (1994), Borland C ++4 Developer's Guide, Sams Publishing, Indianapolis, Indiana.

BRADBURN, N.M., SUDMAN, S. and ET AL. (1979), Improving Interview Method and Questionnaire Design, Jossey-Bass Inc., San Francisco, California, first ed.

BRATKO, I. (1990), PROLOG Programming for Artificial Intelligence, AddisonWesley, Reading, Massachusetts, second ed.

BRIDGE, G.R. and AL, E. (1977), 'Interviewing changes attitudes-sometimes', Public Opinion Quarterly, Spring.

BRODIE, M.L., MYLOPOULOS, J. and SCHMIDT, J.W. (1984), On Conceptual Modelling, Springer-Verlag, New York.

CAREY-SMITH, C., POWLEY, D. and CAREY-SMITH, K. (1993), 'An adaptable health screening questionnaire', In KASABOV, N.K. (Ed.), Artificial Neural Networks and Expert Systems, IEEE Computer Society Press, Dunedin, New Zealand, pp. 259-260.

CONVERSE, J.M. and PRESSER, S. (1986), Survey Questions, Handcrafting the Standard Questionnaire, Quantitative Applications in the Social Sciences, SAGE Publications Inc., Beverly Hills, California.

COPPOLA, W. (1994), 'Test developed to spot likely stroke victims', British Journal of General Practice.

DURKIN, J. (1994), Expert Systems, Design and Development, Macmillian Publishing Company, New York.

KAHN, R.L. and CANNELL, C.F. (1957), The Dynamics of Interviewing: John Wiley and Sons, Inc., New York. 
KUNZE, M. and WOOD, M. (1984), 'Guidelines on smoking cessation', UICC Techincal Report, Vol. 79.

LABAW, P.J. (1980), Advanced Questionnaire Design, Abt Books, Cambridge, Massachusetts.

LOGIE, A.R., MADIRAZZA, J. and WEBSTER, I. (1976), 'Patient evalulation of a computerised quesitionnaire', Computers and Biomedical Research, Vol. 9, pp. 169-176.

LUGER, G.F. and STUBBLEFIELD, W.A. (1989), Artificial Intelligence and the Design of Expert Systems, The Benjamin/Cummings Publishing Company, Inc., Redwood City, California, first ed.

MCAVOY, B.R., POWELL, A.M. and ADAMS, P.J. (1994), Feasibility Study of a Computer Assisted Health Screening Programme for Use in General Practice Settings, Technical Report, RNZCGP Research Unit, Department of General Practice, School of Medicine, University of Auckland.

MOUGHAN, C.J. (1988), 'Prevention in general practice', The New Zealand Family Physician, Summer.

OPPENHEIM, A.N. (1966), Questionnaire Design and Attitude Measurement, Basic Books, Inc., New York.

PARSAYE, K. and CHIGNELL, M. (1988), Expert Systems for Experts, John Wiley and Sons, Inc., New York.

RALSON, A. (1971), Introduction to programming and computer science, McGraw-Hill Inc.

SIWATIBAU, R. (1994), QUEST Business Plan, Master's thesis, Univeristy of Canterbury.

SKINNER, H.A. and ALLEN, B. (1983), 'Does the computer make a difference. computerized versus face-to-face versus self-report assessment of alcohol, drug and tobacco use', Journal of Consulting and Clinical Psychology, Vol. 51, No. 2, pp. $267-275$.

SKINNER, H., ALLEN, B., MCINTOSH, M. and PALMER, W. (1985), 'Lifestyle assessment: Applying microcomputers in family practice', British Medical Journal, Vol. 290, January, pp. 212-214.

SLACK, W.V., HICKS, G.P., REED, C.E. and VAN CURA, L.J. (1966), 'A computerbased medical-history system', The New England Journal of Medicine, Vol. 274, No. 4, January, pp. 194-198. 
WEISS, S.M. and KULIKOWSKI, C.A. (1984), A Practical Guide to Designing Expert Systems, Rowman and Allanheld, Totowa, New Jersey.

YOSHIDA, A., HAGITA, Y., YAMAZAKI, K. and YAMAGUCHI, T. (1993), 'Which do you feel comfortable, interview by a real doctor or by a virtual doctor?', IEEE International Workshop on Robot and Human Communication. 



\section{Appendix A}

\section{DOMAIN DECLARATIONS}

The following domain declarations are taken from the file 'quest.dom'. These define all the variable 'types' used in the entire QUEST project. For the purpose of clarity the domains are grouped together and are commented as groups.

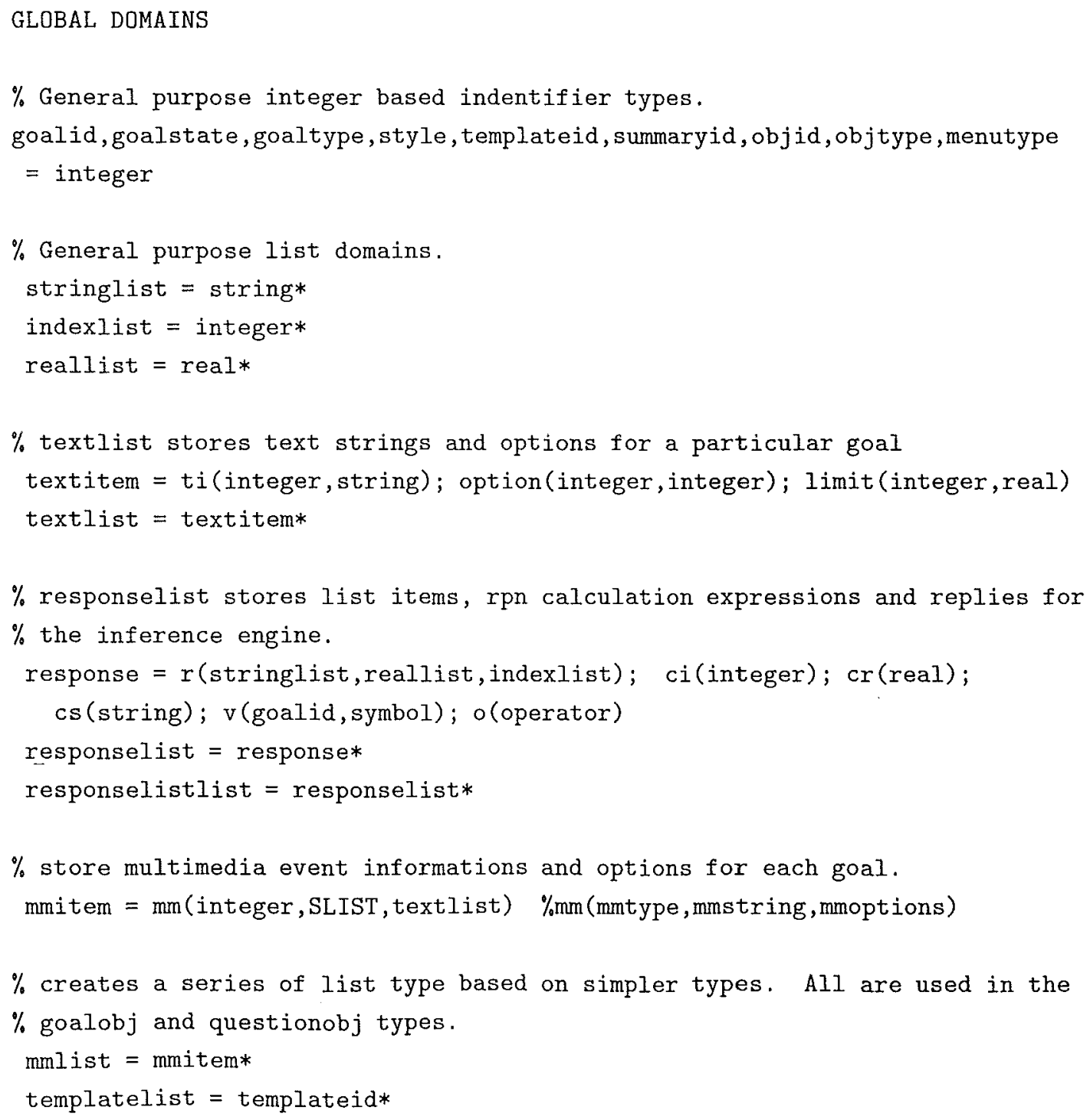


summarylist $=$ summaryid*

goallist $=$ goalid $*$

$\%$ The following two objects completely describe a goal. The fileobj holds

$\%$ questionnaire options and id lists for all the objects in the questionnaire. goalobj $=$ g(goalid, goaltype, goalid, goallist)

questionobj = q(goalid, goaltype,textlist, responselist, mmlist, templateid)

fileobj = $i$ (goallist, templatelist, summarylist, textlist)

$\%$ The following five objects are used within the templateobj object. A $\%$ list of templateobj's make up a template description of the user interface $\%$ for a goal object.

fnt $=f$ (string, integer)

objextra $=\operatorname{text}($ string) $;$ select $($ COLOR); none

object $=$ obj (objid, objtype, RCT, fnt, COLOR, COLOR, style, objextra)

objidlist $=$ objid*

objectlist $=$ object $*$

templateobj $=t$ (templateid,string, goaltype, integer, string, objectlist)

$\%$ The following 6 objects provide the basis for each summary entry.

sumentry = se(goalid, BOOLEAN, string, string, style)

sumentrylist $=$ sumentry*

sumprint $=\operatorname{sp}($ BOOLEAN, BOOLEAN, string, integer $)$

sumfile = sf(BOOLEAN, string,string) \% directory, filename template

summaryobj $=s$ (summaryid, string, integer, sumprint, sumfile, sumentrylist)

summaryobjlist $=$ summaryobj*

$\%$ The next five objects are used when evaluating RPN expressions.

operator $=$ one_ary $($ symbol $) ;$ two_ary (symbol); func (symbol); special (symbol)

funccalc = determ real (real) - (i) \% func calculations predicate domain

twovarcalc $=\operatorname{determ}(r e a l, r e a l$, response $)-(i, i, 0)$

twoidxcalc = determ (integer, integer, response) - (i,i,o)

twostrcalc $=$ determ (string, string, response) $-(i, i, 0)$

$\%$ The three reply objects are used to store the respondent's replies

replyobj = ro (goalid, goalstate, responselist)

replyobjlist $=$ replyobj*

rawreplyobj $=\operatorname{rr}$ (integer, integer, integer, integer, integer, replyobjlist)

$\%$ day, month, year, hour, min, reply list

$\%$ these final domains are used for file I0 operations

db_selector $=$ qstfile ; qstdb; qstreplies

file = logfile; tempoutfile; summaryfile 


\section{Appendix B}

\section{GLOSSARY OF TERMS}

Author The person who defines the form and context of the questionnaire.

AVI File A file format for storing digitised sound and video information.

Block The name of the fundamental question building block in the Question Order flow model.

Child Goal A goal which provides information for a parent goal which is higher up in the questionnaire tree structure.

Command Enabling The process of enabling user interface controls in response to some user action.

Database File A file that contains the all the reply files for a particular questionnaire.

Expert System A knowledge based computer program that provides 'expert quality' solutions to problems in a specific domain.

External Database A binary file format used by the PDC Prolog language for storing information in an efficient database structure.

Goal The name of the fundamental question building block in the Required Information flow model.

Inference Engine The part of a Expert System which applies it's knowledge to the solution of the problem.

Internal Database An database storage method used by PDC Prolog language to maintain information during a program's execution. Can also be saved as text based file format.

Knowledge Representation The study of how information can be represented and stored within a computer system.

Light Pen A device that allows the user to draw on or point at a computer screen. The computer then recognises the point of contact. 
Link Another fundamental building block within the Question Order flow model.

Microsoft Windows An operating system shell that incorporates a Graphical User Interface.

MIDI File A common format for compactly storing musical information.

Multimedia The use of many different media to deliver information.

OWL The Borland Object Windows Library. A C++ application framework library for programming Windows applications.

PDC Prolog A non-standard version of Prolog designed to run on IBM compatible computers. A language based on logic and symbolic programming.

QuestED Part of the QUEST Questionnaire system. Used to create and edit questionnaires.

QuestEX Part of the QUEST Questionnaire system. Used to display and run questionnaires.

Question Order flow The name of a questionnaire representation model used in the QUEST questionnaire system. Structured a questionnaire around a sequence of questions.

Questionnaire File A file containing the complete description of a questionnaire.

QuestView Part of the QUEST Questionnaire system. Used to summarise and store questionnaire replies.

Reply File A file generated by QuestEX, contains the replies to the questionnaire process.

Require Information flow The name of a questionnaire representation model used in the QUEST questionnaire system. Structured a questionnaire the information dependencies within that questionnaire.

Respondent The person who replies to the questionnaire.

RPN Expression A mathematical expression where the mathematical operators appear after the arguments in the expression.

Sound Card A device for playing digital sound and music waveforms.

Summary A form based report of the respondent's replies.

Template A description of the questionnaire screen for a particular goal type. 
Touch Screen A pen shaped device which recognises when and where are user touches the monitor surface.

Tree View A structured hierarchical tree display of the questionnaire in the Require Information flow model.

Wave File A file containing digitally recorded sound information. 

Appendix C

EXAMPLE QUESTIONNAIRES

C.1 HEALTHCHECK QUESTIONNAIRE 


\section{HEALTH CHECK}

\section{Victoria Medical Centre - P.0. Box 90-099 Auckland - Ph 3076-870}

A Questionnaire.

Please fill in details below where applicable.

\section{SURNAME}

\section{FIRST NAME}

ADDRESS

\section{SUBURB}

CITY P.CODE

SPECIAL ID NO.

D.OF BIRTH $(\mathrm{dd} / \mathrm{mm} / \mathrm{yy}) \perp 1$

AGE YEARS.

\section{OCCUPATION}

HEIGHT (CMS) centimetres

WEIGHT (KGS) kilograms

\section{INSTRUCTIONS}

Circle the answers that are correct for you for the following questions.

\section{GENDER \\ 1. Male \\ 2. Female \\ ETHNIC ORIGIN
1. Caucasian
2. Polynesian
3. Indian
4. Asiatic
5. Other origin.

\section{FAMILY ILLNESS HISTORY}

Does any parent, brother or sister have any of the following health problems, either now, or in the past?
1. Heart attack (coronary) or angina prior to age 60 years
2. High blood pressure
3. Bowel cancer
4. Diabetes
5. Asthma
6. Breast cancer
7. Alcohol problems
3. Melanoma
?. None of the above

\section{PERSONAL HEALTH/ILLNESS}

Have you now, or at any time in the past, any of the following diseases or illnesses?
1. High blood pressure
2. Heart attack/coronary/angina
3. Asthma
4. Cancer (incl. melanoma)
5. Diabetes

Have you now, or at any time in the past 5 years. any of the following diseases or illnesses?

6. Stroke or CVA

7. Epilepsy

8. Nervous breakdown or depression needing medication and/or sick leave in the last 5 years.

9. None of any of the above

\section{CURRENT HEALTH PROBLEMS.} Have any of the following problems worried you in the last month?

Possible Early Cancer Signs

1. Mole that darkens, or grows, itches, or bleeds unexpectedly

2. Unexpected change in bowel habit

3. Sores that do not heal

4. Persistent cough, hoarseness, or coughing up blood

5. Any unusual bleeding or bruising

6. Lump in breast or nipple discharge

7. Loss or gain in weight without a change in diet

8. Passing blood/mucus in bowel motion (faeces/stool)

Other Possible IIIness Signs

9. Chest pain or discomfort with exercise, eating or cold weather

10. Depression or thoughts of suicide

11. Marked excess in thirst

12. Wheeziness - marked \& limiting

13. Unexpected shortness of breath

14. Severe or persistent indigestion

15. Unexplained fainting, vertigo, or dizzy spells

16. Pain that is affecting sleep or limiting daily activities

Copyright 1988 Dr S.J.Culpan Lifelong Health Services 
PERSONAL CANCER RISKS.

Circle any of the following that apply to you.

1. Fair skin that burns easily

2. More than 5 brown raised moles of any size/shape on right arm

3. Exposure to asbestos in past

4. Unsafe exposure to radiation or radio-active materials.

5. Exposure to any chemicals known to cause cancer in some people

6. Don't eat a serving of carrot, pumpkin, squash, or green leafy vegs, at least 4 - 6 times a week

7. Don't eat a serving of cabbage or cauli or broccoli or sprouts at least 4 - 6 times a week

8. Don't get regular cervical (Pap) smears

9. Do not perform breast self exam

10 Eat nitrate foods (salted/pickled/cured) at least twice wkly o.g. salami/pickles/hams

\section{SMOKING}

1. Never smoked

2. Stopped smoking over 10 years ago

3. Stopped smoking 5 - 10 years ago

4. Stopped smoking $0-5$ years ago

5. Smoke up to 10 cigarettes per day

6. Smoke 10 to 20 cigarettes per day

7. Smoke 20 to 40 cigaretles per day

8. Smoke over 40 cigarettes per day

9. Smoke pipe or cigars only

\section{ALCOHOL}

How otten do you drink alcohol?

1. Every day usually

2. 3 - 5 days a week

3. 1 - 2 days a weok

4. Occasionally

5. Never

On average how many alcohol drinks would you have per week? (One drink equals one nip of spirit, 1 glass of wine, 1 can of beer, and 2 - 3 cans of low alcohol beer.)

\section{None}

2. 1 - 7 drinks per week

3. 8 - 14 drinks per week

4. 15 - 28 drinks per week

5. Over 28 drinks per week

\section{CAFFEINE}

Add up the number of cups of coffee, tea, and cola drinks you average a day. (Exclude decaffeinated coffee)

1. 0 - 3 cups per day

2. 4 - 6 cups per day

3. 7 or more cups per day

\section{MEDICATIONS}

Are you taking any medicines prescribed by your doctor or bought from the chemist?

1. Yes

2. No

\section{EXERCISE}

On average, how many times a week do you do exercise for at least 30 minutes on end (hard enough to make you puff and sweat)? e.g jogging, aerobics, brisk walking, swimming \& active sports.

1. Less than once a week

2. 1 - 2 times weekly

3. 3 - 4 times a week

4. More than 4 times a week

\section{SAFETY HABITS}

\section{Driving}

When driving or travelling with someone, do you?

1. Always drive within the speed limit

2. Drive within $5 \mathrm{~km} / \mathrm{hr}$ of limit and decrease speed for poor conditions

3. Often drive over the speed limit

4. Usually drive over the limit

\section{Seat Belts}

When travelling by car do you?

1. Always wear a seat-belt.

2. Usually wear a seat-belt.

3. Often do not wear a seat belt

4. Never wear a seat belt

\section{Driving and Drinking Álcohol}

When driving do you?

1. Never drive after drinking - or never drive - or never drink at all

2. Drive after drinking only low alcohol beer

3. Drive only if you have only drunk up to 1 drink per hour

4. Drive after drinking more than 1 drink/hour

\section{Swimming}

Can you?

1. Swim well even in open sea, \& for more than 100 metres.

2. Swim for a short distance

3. Dog-paddle 10 feet if needed.

4. Manage to float only

5. Not able to swim or float

Resuscitation.

Do you?

1. Have a training in first aid and know how to do mouth to mouth breathing and heart massage.

2. Not know how to do mouth to do mouth breathing and heart massage 


\section{STRESS/DISTRESS}

Circle any of the following that fit the way you have been feeling recently

1. Often depressed or very unhappy.

2. Not able to cope with every day hitches at home or work.

3. Seldom tired or fatigued apart from after hard physical work

4. Difficult to socialise - avoid meeting people.

5. Sleeping under 7 hours a night

6. Don't think I am doing a good job of life at present

7. Enjoy most days - work and home

8. Have no friends or people I can talk to if I need help or advice

9. Desperate for help

10 Have disturbing or unreal thoughts

\section{DIET AND EATING HABITS}

Choose the answer that best fits your average eating habits.

\section{BREAKFAST}

Do you eat a good breaktast -

(Cereal or at least 3 pieces whole-meal bread. optional fruit or fruit juice)

1. Hardly ever

2. About once a week

3. 2 - 4 days a week

4. Almost every day

\section{BREAD, CEREAL \& GRAIN PRODUCT}

Unrefined Whole Grain Foods

High fibre and whole grain breads and rolls, bran or oat based biscuits, pastas,

brown rice, high fibre cereals without sugar (such as oat based, bran, weetbix, cornflakes, muesli \& porridge )

Befined Lew fibre Foeds.

White bread, sugar coated cereals, biscuits, cakes, doughnuts,

white rolls and buns

1. Eat mostly only refined type

2. Eat more refined than whole grain

3. Eat equal amounts of both

4. Eat more whole grain than refined

5. Eat mostly only whole grain type

\section{MEAT POULTRY SEAFOOD}

1. Eat mainly red meat, and or food such as chicken with skin, luncheon or salami. pates, crayfish or prawns.

2. Mostly eat red meat,but aiso eal some white poultry meat or fish

3. Eat equal amounts of red meat \& fish/poultry.

4. Eat mainly fish or skinless poultry.

5. Rarely eat any meat, poultry or fish - mainly vegetarian.

\section{COOKING METHODS}

Choose the way you tend to cook, or the foods you would choose to eat

Low fat loods

Bake, broil, steam, or grill. Use vegetable oils and sparingly, no butter, fow eggs added, low-fat dressings.

High fal foods Frying or roasting, deep frying, batters, gravies and rich sauces, added cheese. Solid fats and butter to cook with. Eggs added

1. Eat mostly only high fat type

2. Eat more high fat than low fat

3. Eat equal amounts of both

4. Eat more low fat than high fat

5. Eat mostly only low fat type

\section{EGGS}

Mark the number of eggs you eat on average per week, (Add those used in cooking if possible.)
1. 0 - 3 eggs per week
2. 4 - 7 eggs per week
3. 8 - 14 eggs per week
4. More than 14 per week

\section{DAIRY PRODUCTS}

Choose the answer that best fits your eating patterns and habits.

\section{Low Fat Dairy Products}

Low-fat milk/cheeses/yoghurts, soya products, cottage cheese.

High Fat Dairy Products

Hard cheeses, cheddar, colby, and processed cheeses, cream, ice-cream, whole-fat milk.

1. Eat mostly only low fat type

2. Eat more low fat than high fat

3. Eat equal amounts of both

4. Eat more high fat than low fat

5. Eat mostly only high fat type

6. Eat no dairy products 
FRUITS \& VEGETABLES

On average, how many servings of fruit and vegetables do you eat per day?

(1 serving equals one piece of fruit or a small cup of vegetables, or 1 glass fruit. juice)

1. Up to one serving a day

2. 2 servings per day

3. 3 servings per day

4. 4 servings per day

5. 5 or more servings per day

\section{BUTTER/MARGARINE SPREADS}

When using the following, do you usually use and eat?

1. Butter

2. Some butter /some margarine

3. Mainly margarine

4. Only low fat/low cholesterol high polyunsaturated margarine

5. Do not use butter or margarine.

\section{SALT}

Do you usually?

1. Add salt at table and cooking OR - eat crisps, salted nuts, pickles, several days every week.

2. Add salt to cooking only $O R$ - lightly salt at table only and only occasionally eat crisps and nuts.

3. Not add salt to cooking or at table and avoid salty foods.

\section{TAKEAWAYS}

How often do you eat takeaways such as hot chips, battered and deep fried foods. hamburgers, fried chicken, pies and ice-creams or thick-shakes?

1. Most days

2. 2 - 3 times a week

3. No more than once a week

4. Less than once a week

5. Rarely or never

\section{BETWEEN MEAL SNACKS}

Do you snack between meals? (biscuits, muesli or snack bars)

1. Yes

2. No
HEALTH DATA ENTRY

(To be filled in by test personnel only)

Height (not in shoes) cms

Weight (clothed) kgs

Frame Size
1. Small
(circle choice)
2. Medium
3. Large

Resting pulse (beats/min)

Blood Pressure (lying or seated)

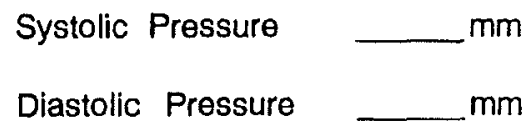

Flexibility $\mathrm{cms}$

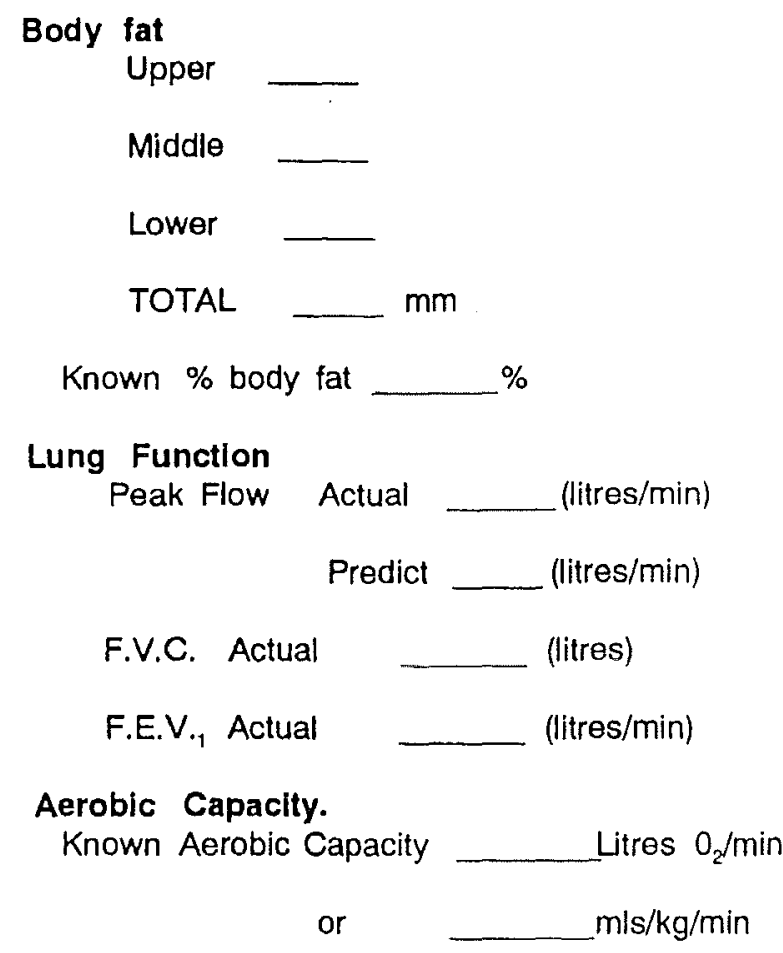

Bicycle Ergometry One Stage

Watts

Heart Rate

BLOOD TESTS.

Total Cholesterol (mmol/l)

HDL Cholesterol $(\mathrm{mmol} / \mathrm{l})$

Glucose (mmol/l)

G.G.T. $(\mathrm{mmol} / \mathrm{l})$ 


\section{C.2 STROKE QUESTIONNAIRE}

\section{Test developed to spot likely stroke victims}

Most high risk stroke paclents attending general practice surgeries could be identilied using a simple scoring system, say speciat ists In the UK.

Using age, systolic blood pressure, current cigarettc consumption and evidence of anginal chest pain, re searchers were able to pre dict 82 per cent of all strokes over live years in thie $\mathrm{llgh}$ risk group.

The patient needs no extra investlgation or $d$ :tailed medical history so the test could be performed by prac- tice nurses at low cost.

Dr Will Coppola, study coauthor and clinical research fellow at London's Royal Free Hospltal, said the system, based on analysis of data of 7735 men aged 40 to 59 years in the British Reglonal Heart Study, could also be used lor women.

"It will enable GPs to plck out peoplc at particularly high risk and concentrale preventlve action on those who need it most," said $\mathrm{Dr}$ Coppola.

The paper, published in the April edition of the British

STROKE PREVEMUIOH

\section{Scoring systems}

- Multiply age of patlent by nine

- Add 2.85 x systolic blood pressure

- Add 70 ir anglna is presert

- Add 90 ir the patlent smokes between one and 20 cigarettes a day

- Add 130 il patient smokes more than 20 cigaret :es a day

- Target patients scoring over 1001

Joumal of Generul Practice. says Intervention for the 20 per cent of patients at the highest risk would provide the majority of stroke prevention required.

The rescarchers recommend patients scoring over 1001 are targeted for intervention treatment.

They call lor doctors to lo cus on manaying smoking and hypertension and stress the limportance of avoidling Inacetivity. This, claim the researchers, is associated with up to a three-lu'd rise in stroke risk

Their study found a combination of smoking and liypertension, while not as sensitive as the scoring system, was a better indicator of risk than any single lactor. 


\section{C.3 SMOKING QUESTIONNAIRE}

PREDICT IVE SCALE OF DEPENDENCE A.ND MOT IVATION

\section{Dependence}

Always Frequentiy Occasionally Seldom Never

A: Ifeel irritable \& moody when I haven't smoked for a while: 5

$$
4
$$

3

21

B: I usually have a olgarette within $3 \emptyset$ mins of waking.

$5 \quad 4$

3

21

C: I develop a craving for a clgarette when without them.

4

3

2 1

Motivation to Quit

\begin{tabular}{ll} 
Strongly- Milalg- \\
Agres & Agree \\
\hline
\end{tabular}

D: I am confident of quitting in tine next 4 whs.

$$
1
$$

2

3

4

$\mathrm{E}$ : Bj continulng smoking I risk òveloping serious heart and iung problems.

$F$ : I very much rant to quit smoklng immediately. 1

2 3 4 $A+B+C \geqslant 9=$ HIGHLY DEPENDENT D E + F $\$ 4=$ WELI MOTIVATED 


\section{C.4 READINESS TO CHANGE QUESTIONNAIRE}

The following questionnaire is designed to identify how you per sonally fecl about your drinking right now. Please read each of the questions below carefully, and then decide whether you agree or disagree with the statements. Please tick the answer of your choice lo cach question. Your answers are completely private and confidential.

1. I don't think I drink too much.

Strongly Dissgrce Unsure Agree Strongly

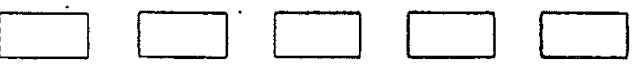

2. I am urying to drink less than I used to.

3. I onjoy my drinking, but sometimes I drink too much.
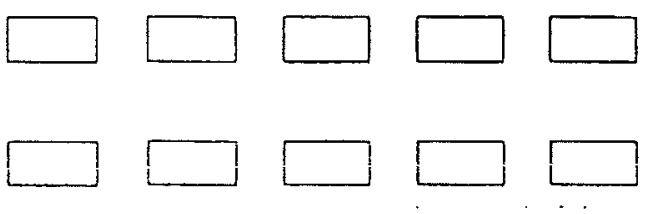

4. Sometimes I think I should cut down on my drinking.
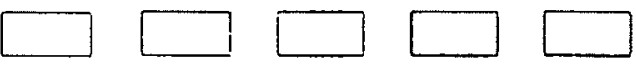

5. Il's a waste of time thinking about my drinking.
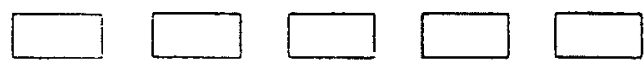

6. I have just recently changed my drinking habits.
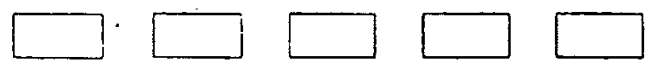

7. Anyone can talk about wanting to do soneching about drinking, but I am actually doing
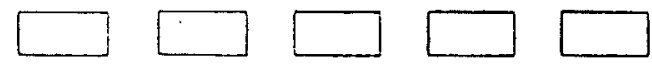
something about it.

8. I am at the stage where I should think about drinking less alcohol.
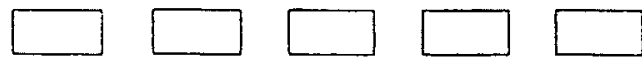

9. My drinking is a problem cometimes.

10. There is no need for m? 0 think about ch:ınging my drinking.

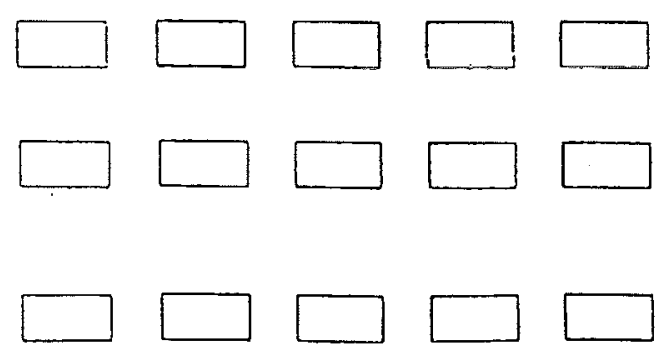

11. I am actually changing my drinking habits right now.

12. Drinking less alcol: $\quad$ ! be pointless for me.
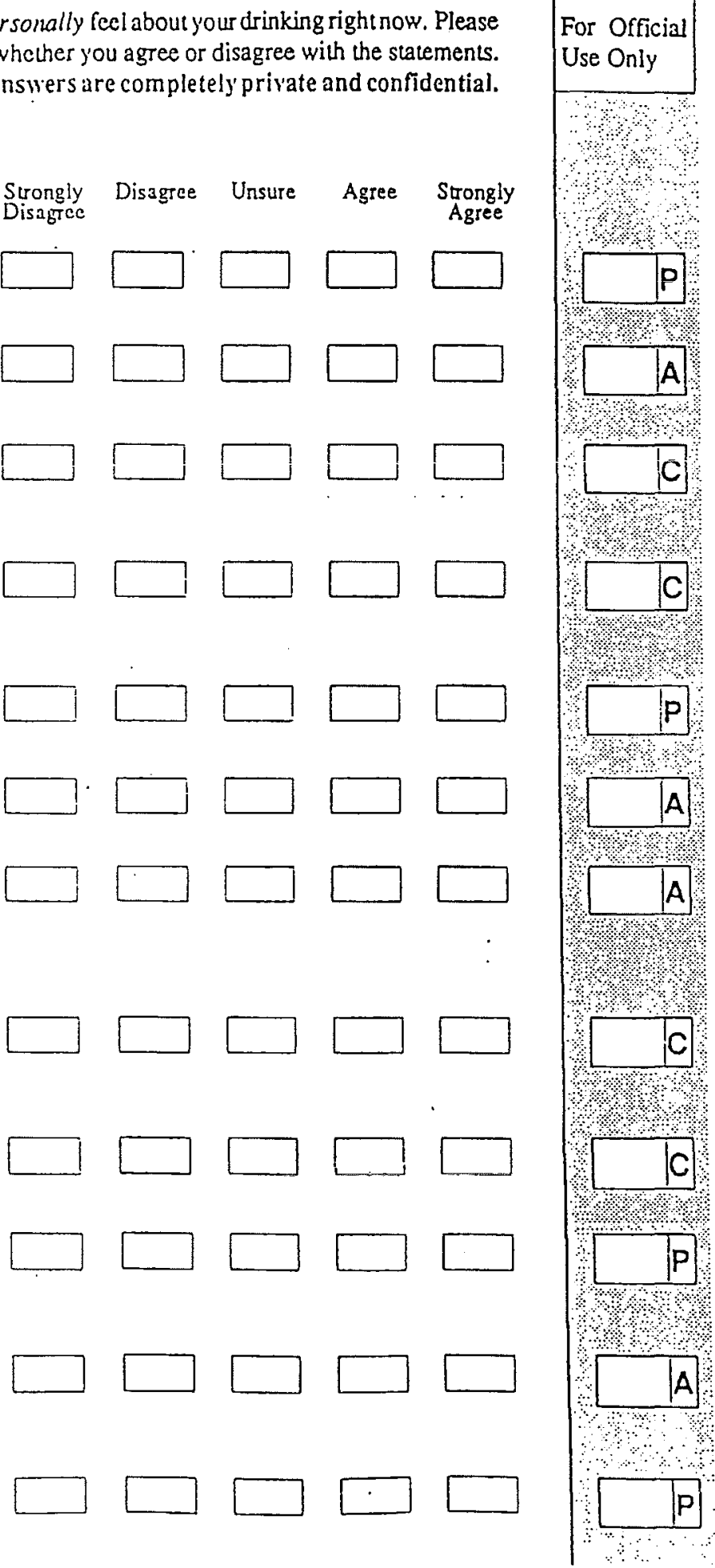


\section{Scoring the Readiness to Change Questionnaire.}

The Prccontcmplation items are numbers $1,5,10 \& 12$, the Contcmplation items are numbers $3,4,8 \& 9$, and the Action ilems are numbers $2,6,7$ \& 11 . All items are to be scored on a 5-point rating scale ranging from:

\begin{tabular}{|ll}
\hline-2 & Strongly disagrec \\
\hline-1 & Disagree \\
\hline 0 & Unsure \\
+1 & Agree \\
+2 & Strongly agree
\end{tabular}

To calculate the score for each scale, simply add the item scores for the scale in question. The range of each scale is -8 through 0 to +8 . A negative scale score reflects an overall disagreement with items measuring the stage of change, whereas a positive score represents overall agreement. The highest scale score represents the Stage of Change Designation.

Nole: If two scale scores are equal, then the scale farther along the continuum of change (Precontemplation - Contemplation - Action) represents the subject's Stage of Change Designation. For example, if a subject scores 6 on the Precontemplation scale, 6 on the Contemplation scale and -2 on the Action scale, then the subject is assigned to the Contemplation stage.

Note that positive scores on the Precontemplation scale signify a lack of readiness to change. To obtain a score for Precontemplation which represents the subject's degree of readiness to change, directly comparable to scores on the Contemplation. and Action scalcs, simply reverse the sign of the Precontemplation score (see below).

If one of the four items on a scale is missing, the subject's score for that scale should be pro-rated (i.e. multiplied by 1.33). If two or more items are missing, the scale score cannot be calculated. In this case the Stage of Change Designation will be invalid.

\section{Scale Scores}

Precontemplation Score

Contemplation Score

Action Score

\section{Readiness to Change}

Prccontemplation

Contemplation

Action (reverse score)

(same score)

(same score)

Stage of Change Designation

(P,C or A). 


\section{C.5 AUDIT QUESTIONNAIRE}

\section{The AUDIT Questionnaire}

1. How often do you have a drink containing atcohol?
(0) Never
(1) Monthly
(2) Two to four
(3) Two to three
(4) Four or more
or less
limes a month
times a week
times a week

2. How many drinks containing alcohol do you have on a typical day when you are drinking? [Code number of slandard drinks]"
(O) 1 or 2
(1) 3 or 4
(2) 5 or 6
(3) 7 to 9
(4) 10 or more

3. How othen do you have six or more drinks on one occasion?
(0) Never
(1) Less than
(2) Monthly
(3) Weekly
(4) Daily or
almost daily

4. How otten during the last year have you found that you were not able to stop drinking once you had started?
(0) Never
(1) Less than
(2) Monthly
(3) Weekly
(4) Daily or monthly
atmost daily

5. How often during the last year have you failed to do what was normally expected from you because of drinking?
(0) Never
(1) Less than
(2) Monthly
(3) Weekly
(4) Daily or monthly
almost daily

6. How often durng the last year have you needed a first drink in the morning to get yourself going after a heavy drinking session?
(0) Never
(1) Less than
(2) Monthly
(3) Weekly
(4) Daily or monthly
almost daily

7. How often during the last year have you had a leeling of guilt or remorse after drinking?
(0) Never
(1) Less than
(2) Monthly
(3) Weekty
(4) Daily or monthly
almost daily

8. How often during the last year have you been unable to remember what happened the night betore because you had been drinking?
(0) Never
(1) Less than
(2) Monthly
(3) Weekly
(4) Daily or monthly

9. Have you or someone else been injured as a result of your drinking?
(O) No
(2) Yes, but not in
(4) Yes, during the last yea the last yea

10. Has a relative, friend, doctor, or other health worker been concerned about your drinking or suggested that you should cut down?
(0) No
(2) Yes, but not in
(4) Yes, during

the last year

the last yea 


\section{C.6 HEALTHCHECK STAFF QUESTIONNAIRE}

\section{HEALTHCHECK}

PRACTICE STAFF QUESTIONNAIRES - 1 AND 2 (Pre and Post Test)

AGE: years

GENDER: $\quad M \square$

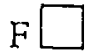

ROLE IN THE PRACTICE

$\square$ Receptionist
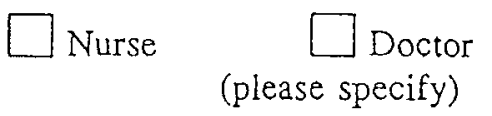

$\square$ Other (please specify)

1 How comfortable do you feel with the use of computers? (please circle)

$\begin{array}{lllllllll}\begin{array}{l}\text { Strong } \\ \text { discomfort }\end{array} & 1 & 2 & 3 & 4 & 5 & 6 & 7 & \begin{array}{l}\text { Very } \\ \text { comfortable }\end{array}\end{array}$

$2 \quad$ How often do you use a computer?

(i) daily

(ii) two to three times per week

(iii) once weekly

(iv) less than weekly

3 Do you see computers as a useful instrument in Health education?

$\begin{array}{lllllllll}\begin{array}{l}\text { Nor } \\ \text { useful }\end{array} & 1 & 2 & 3 & 4 & 5 & 6 & 7 & \begin{array}{l}\text { Very } \\ \text { useful }\end{array} \\ \end{array}$


$4 \quad$ Which age group do you see as most likely to use computers
Age
$18-30$
$30-45$
$45-60$
$60+$

5 In your contact with patients generally how often do you educate or advise them about health risks and lifestyles as they affect their health? Would you say
(i) All the time
(ii) Most of the time
(iii) Some of the time
(iv) Rarely or never
(v) Not applicable

6 At the present time, how important do you see health promotion as an aspect of this practice.
Very 12
$3 \quad 4 \quad 5$
6
7 Very
important
unimportant

7 The following are behaviours that some health professionals believe to be related to health. How important do you think the following behaviours are in promoting the health of the average person? (Please circle one number for each.)

(i) Not smoking

$\begin{array}{llllll}\text { Very important } & 1 & 2 & 3 & 4 & \text { Very unimportant }\end{array}$

(ii) Exercising regularly

$\begin{array}{llllll}\text { Very important } & 1 & 2 & 3 & 4 & \text { Very unimportant }\end{array}$

(iii) Drinking alcohol moderately

$\begin{array}{llllll}\text { Very important } & 1 & 2 & 3 & 4 & \text { Very unimportant }\end{array}$

(iv) not drinking alcohol at all

$\begin{array}{llllll}\text { Very important } & 1 & 2 & 3 & 4 & \text { Very unimportant }\end{array}$ 
(v) not using cannabis

$\begin{array}{llllll}\text { Very important } & 1 & 2 & 3 & 4 & \text { Very unimportant }\end{array}$

(vi) avoiding excess calories

$\begin{array}{llllll}\text { Very important } & 1 & 2 & 3 & 4 & \text { Very unimportant }\end{array}$

(vii) reducing stress

$\begin{array}{llllll}\text { Very important } & 1 & 2 & 3 & 4 & \text { Very unimportant }\end{array}$

(viii) not using narcotics or other abusable substances

$\begin{array}{llllll}\text { Very important } & 1 & 2 & 3 & 4 & \text { Very unimportant }\end{array}$

8 If you are a member of Clinical Staff indicate how much you agree or disagree with each of the following statements about working with problem drinkers. "Problem drinkers" refers to people with harmful alcohol use, but excludes late-stage alcoholics. (Please circle one number for each question.)

I feel I know enough about the causes of drinking problems to carry out my role when working with problem drinkers.

$\begin{array}{lllllllll}\text { Strongly } & & & & & & & & \begin{array}{l}\text { Strongly } \\ \text { disagree }\end{array} \\ \text { agree } & 1 & 2 & 3 & 4 & 5 & 6 & 7 & \end{array}$

I feel I can appropriately advise my patients about drinking and its effects.

$\begin{array}{lllllllll}\begin{array}{l}\text { Strongly } \\ \text { agree }\end{array} & 1 & 2 & 3 & 4 & 5 & 6 & 7 & \begin{array}{l}\text { Strongly } \\ \text { disagree }\end{array}\end{array}$

I feel I have the right to ask patients questions about drinking when necessary.

Strongly

$\begin{array}{lllllllll}\text { agree } & 1 & 2 & 3 & 4 & 5 & 6 & 7 & \text { disagree }\end{array}$

I want to work with problem drinkers

Strongly

$\begin{array}{lllllllll}\text { agree } & 1 & 2 & 3 & 4 & 5 & 6 & 7 & \text { disagree }\end{array}$


Pessimism is the most realistic attitude to take with problem drinkers.

Strongly

$\begin{array}{lllllllll}\text { agree } & 1 & 2 & 3 & 4 & 5 & 6 & 7 & \text { disagree }\end{array}$

I feel I do not have much to be proud of when working with problem drinkers

$\begin{array}{lllllllll}\text { Strongly } \\ \text { agree } & 1 & 2 & 3 & 4 & 5 & 6 & 7 & \begin{array}{l}\text { Strongly } \\ \text { disagree }\end{array}\end{array}$

All in all, I'm inclined to feel $I$ am a failure with problem drinkers

$\begin{array}{llllllllll}\text { Strongly } & & & & & & & \text { Strongly } \\ \text { agree } & 1 & 2 & 3 & 4 & 5 & 6 & 7 & \text { disagree }\end{array}$

In general, it is rewarding to work with problem drinkers

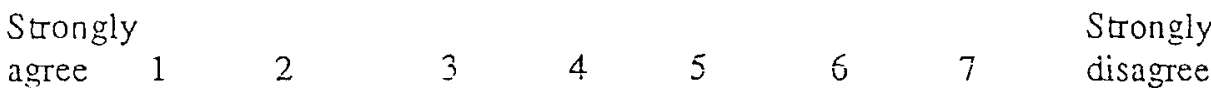

In general, I like problem drinkers.

$\begin{array}{lllllllll}\text { Strongly } & & & & & & & & \begin{array}{l}\text { Strongly } \\ \text { agree }\end{array} \\ \text { disagree }\end{array}$

$9 \quad$ How much do you agree with the following statements? (Please tick the appropriate box)

(i) Information on health status helps people make healthy choices.

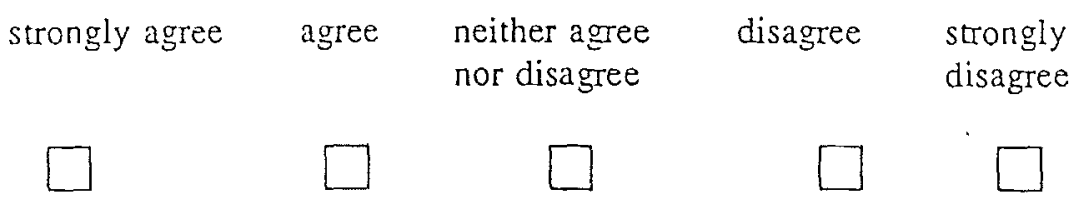

(ii) Patients want information about health status.

$\begin{array}{ccccc}\text { strongly agree } & \text { agree } & \begin{array}{c}\text { neither agree } \\ \text { nor disagree }\end{array} & \text { disagree } & \begin{array}{l}\text { strongly } \\ \text { disagree }\end{array} \\ \square & \square & \square & \square & \square\end{array}$


(iii) GP's have no part to play in the treatment of drug and alcohol problems.

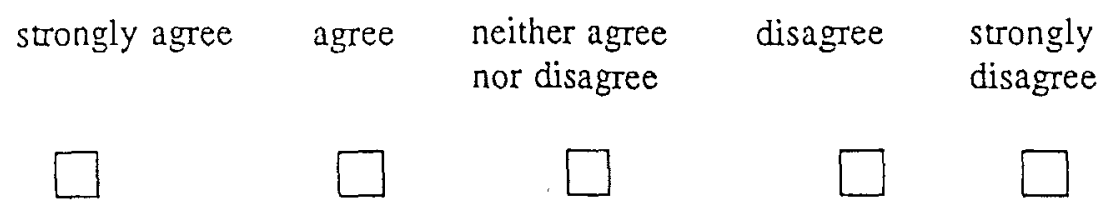

(iv) Public health education has only worked well with well educated people.

$\begin{array}{llll}\text { strongly agree agree } & \begin{array}{l}\text { neither agree } \\ \text { nor disagree }\end{array} & \text { disagree } & \begin{array}{l}\text { strongly } \\ \text { disagree }\end{array}\end{array}$

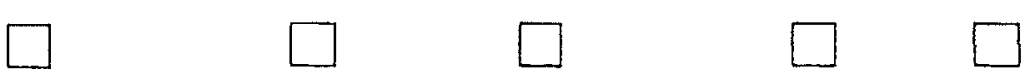




\section{C.7 TEENAGE HEALTH AND FITNESS QUESTIONNAIRE}

\section{TEENAGE HEALTH \& FITNESS QUESTIONNAIRE}

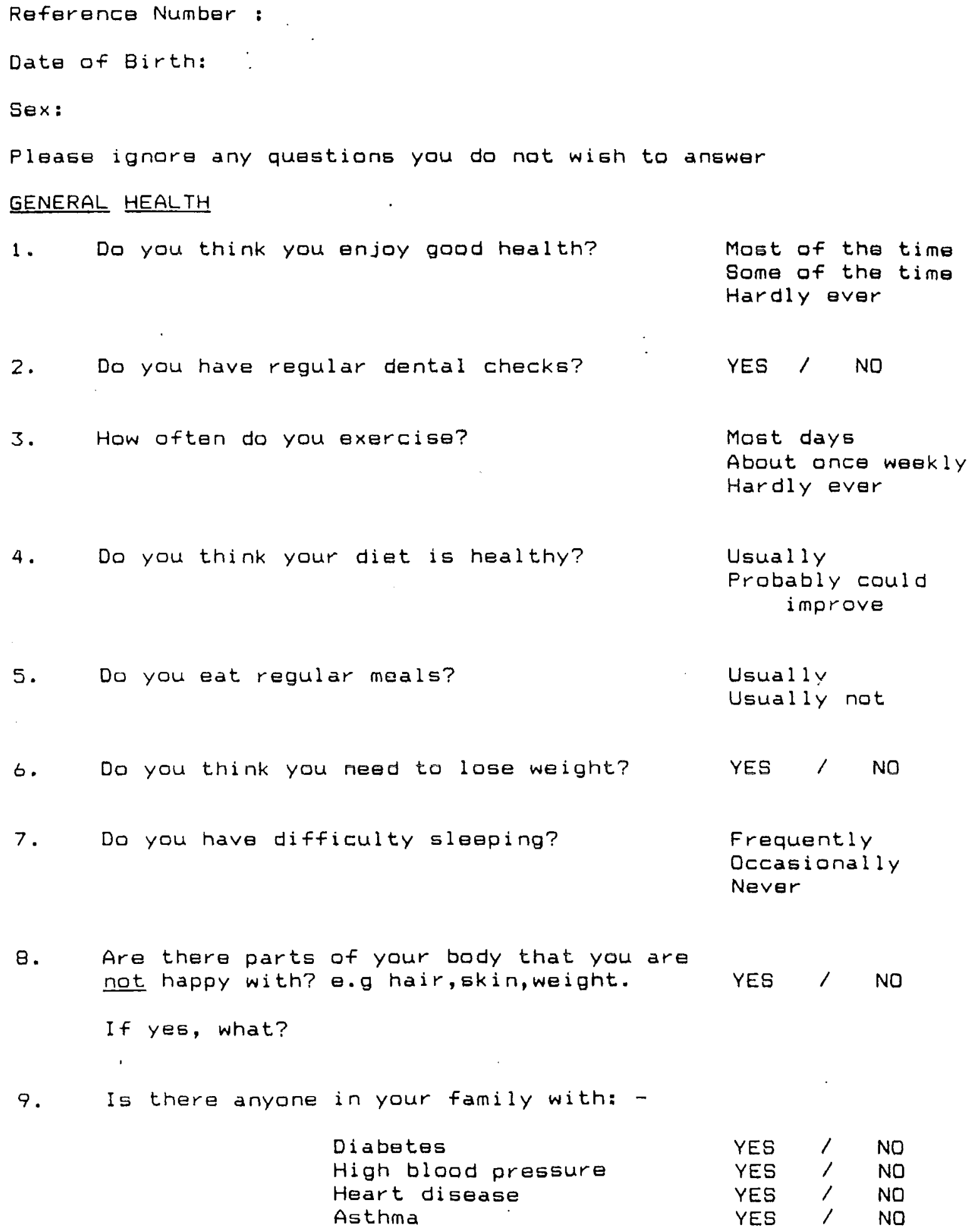

$\begin{array}{lll}\text { YES } & \text { I } & \text { NO } \\ \text { YES } & 1 & \text { NO } \\ \text { YES } & \text { NO } \\ \text { YES } & \text { N } & \text { NO }\end{array}$


HEALTH \& RELATIONSHIPS

1. Do you feel you get enough support from your family

your friends

Some of the time

Hardly ever

Most of the time

Some of the time

Hardly ever

others

Most of the time

Some of the time

Hardly ever

2. Do you feel good about yourself?

Most of the time

Some of the time

Hardly ever

3. Do you find yourself feeling down?

Never

Sometimes

often

4. Do you feel lonely?

Never

Sometimes

Often

5. Do you have a close friend?

YES $/$ NO

b. Do you feel free to make your own decisions regarding relationships?

Usually

Eometimes

Never

7. Are you concerned about the possibility of

pregnancy
sexually transmitted diseases
AIDS

YES $/$ NO

YES $r$ NO

YES $/$ NO

8. Do you feel you have been "used" in any previous sexual situation?

Sometimeśs

Aefepionally

9. Do you feel you need any information on contraception?

YES / NO 


\section{TEENAGE HEALTH \& FITNESS QUESTIONNAIRE}

\section{HEALTH \& PERSONAL SAFETY}

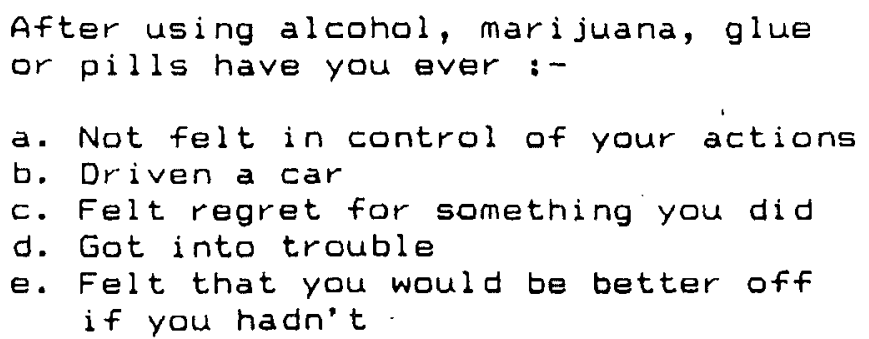

$\begin{array}{lll}\text { YES } & \text { NO } \\ \text { YES } & \text { NO } \\ \text { YES }, & \text { NO } \\ \text { YES }, & \text { NO } \\ \text { YES , NO }\end{array}$

2. Have you been a passenger in a car when the driver has cansumed alcohol, marijuana, glue or pills?

YES / ND

3. Do you smoke cigarettes?

If so, how many per day on average?

Do you want help to give up smoking?

YES $\quad / \quad$ NO

...........

YES

\section{HEALTH INFORMATION}

1. When you ride a bike do you wear a helmet

Always

Sametimes Never

2. When you are in a car do you make sure you wear a seat belt?

Always

Sometimes Never

3. Have you access to enough health information?

YES

NO

4. Do you have any worries about cancer or any other disease?

YES / NO

If yes, what?

5. Do you have any health concerns or problems you wish to discuss further?

If yes, what?

Did you find this questionnaire helpful? 
Appendix D

THE USER GUIDES

D.1 QUESTED USER GUIDE 



\section{The QUEST Questionnaire System}

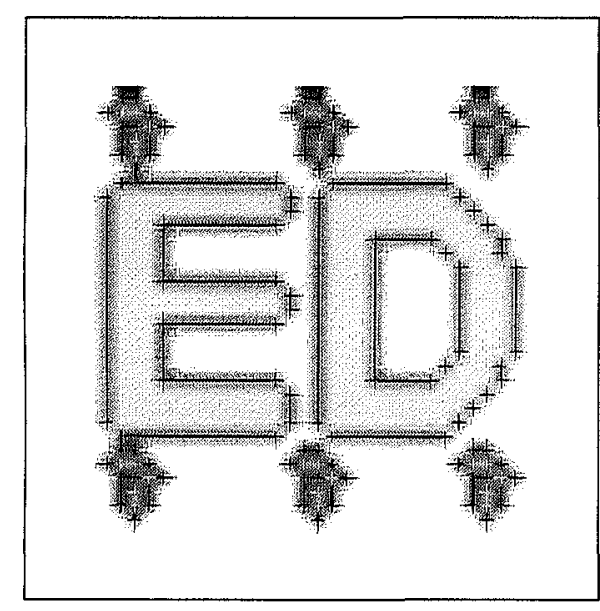

\section{QuestED User Guide}

\section{Version 2.0}

\section{QuestED User Guide.}

This Users guide is divided into four parts. The first part introduces the concepts behind the QUEST Questionnaire System. The three following parts describe the use of the Questionnaire Editor, the Summary Editor and the Template Editor.

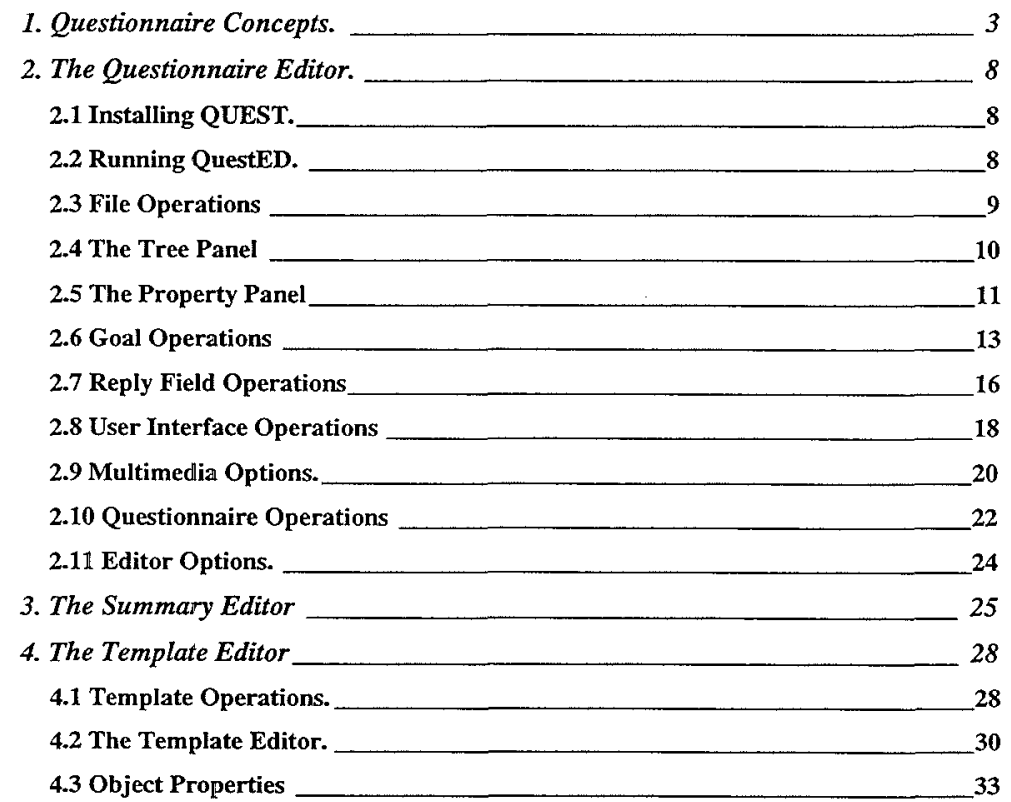




\section{Questionnaire Concepts.}

The QUEST Questionnaire system consists of three main components. Figure shows a simple relationship between these components. The compenents are,

QuestED - This is a tool that allows a questionnaire author to manipulate questionnaire files. The questionnaire file completely describes the content appearance and behavior of the questionnaire. The structure of a questionnaire consists of a hierarchy tree. Each node of the tree is called a goal. Each goal's aim is

QuestEX - This component plays the questionnaire file generated by QuestED Running the questionnaire involves displaying to the questionnaire respondent each questionnaire goal. The respondent then enters an appropriate reply to that questionnaire goal. Each goal need not necessarily be a question; it may be a section heading or an information goal. The respondent's replies are then saved and summarized according to the summary defined in QuestED.

QuestView - This program allows the saved reply files to be viewed at a later date. The questionnaire summaries for that file can be regenerated if necessary. QuestView can also produce an overall summary of a series of individual summaries. This overall summary can show a statistical breakdown of for each of the questions in the questionnaire.

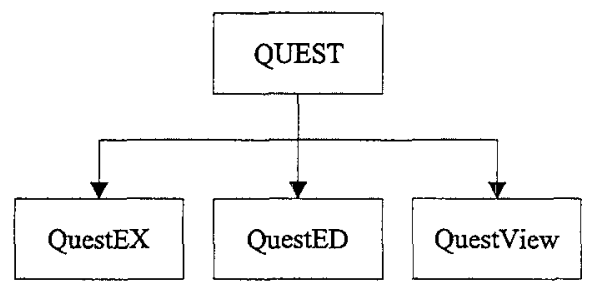

Figure 1: The System Overview.

The fundamental unit in the QUEST system is the question goal. A goal's purpose is to obtain a piece of information and then past it on to a parent goal. The goal may obtain its information from one of two places, the questionnaire respondent, or another goal. The goal may pass the information it has obtained on to a summary and/or another goal. Each piece of information consists of three parts, a text string, an integer index, and a real variable. This allows for up to three representations for a reply. For example, the answer to the question 'How many days are there in a week?' can be represented as " 7 ", 7 , and 7.00
From this, there must be number of different basic goal types. The two main types are Question goals and Control goals. Question goals simply ask the respondent for the answer to a question. The types of Question goals are,

1. Text Entry. This goal asks the respondent to enter a text reply. An example being 'What is your name?' The answer is stored as a text string.

2. Numeric Entry. This goal asks the respondent to enter a numeric reply. Only a numeric reply is considered valid, and the respondent can not continue until a valid number is entered. Upper and lower limits can be placed on the entry. For example the question, 'How many days of sick leave did you take last year?' can use the limits 0 and 365 to ensure a valid reply. The answer is stored as a text string and as a real variable.

3. Single Select List. This goal displays a list of pre-determined items to the respondent. The respondent must choose one of the items from the list. The chosen item's list index is stored as the integer index and the item's text is stored as the text string. A 'value' may also be associated with each item in the list. For example the associated value maybe a weighting that can be used in a calculation. The chosen item's associated value is stored as the real variable.

4. Multi Select List This goal is similar to the Single Select List in that a list of items is presented to the respondent. However the respondent may choose any number of items from the list, including none. Again each value may have an number of items
associated value.

The Control goals get their name from the fact they control other goals. These other goals are called sub goals as they are effectively subordinate to the control goal. Alternatively the control goal may be viewed as a 'Parent' goal to it's 'Child' goals The purpose of the Control goals is to organize the questionnaire into a meaningful structure in the context of a particular questionnaire. Examples of this are the grouping of a series of goals, and the conditional execution of a goal. The types of grouping of a series
Control goals are,

1. Group Goal. The group goal acts to 'group' together a series of sub goals together. For example, a questionnaire has a number of well-defined sections of questions. A group goal can be used as the section introduction for each of these sections of questions. The group goal is the only goal that has an optional user interface. By using a user interface the group goal can act as section introduction as in the previous example. The group sub goals are processed. However when used without a user interface, the group goal can invisibly group goals together. The value of this will become apparent when condition goals are discussed.

2. Calculation Goal. The calculation goal provides the facilities to evaluate arithmetic, logical and relation expressions. These expressions may consist of regular calculations and goal references. The goal references refer to the replies of the calculation's sub goals. For example, a calculation goal may add the respons guestions. The calculation goal has the ability to proce 
the part of a response, text, index and variable. For example, an index maybe added to a variable. As long as the operation in valid, the correct conversion between types will occur. It is obvious that a text string can not be added to a real variable for example. The calculation goal has no user interface and executes transparently to the respondent.

3. Info Goal. The info goal's purpose is to display some useful information to the respondent. For example, it may display information on why a series of questions is being asked. It is similar to both the Group goal and the Calculation goal in certain respects. Like the group goal it displays information to the respondent, and does not require an answer or reply. However the info goal's user interface does not display until after it's sub goals have been executed. This is because it may use the responses from a number of sub goals in the information it gives to the respondent. Like a calculation goal it may reference its own sub goals. Thus it processes the sub goals and then displays the information in the user interface.

4. Condition Goal. The condition goal is different to all other goal types as it acts as a sub goal to all other types of goal. As a special sub goal, the condition goal is executed BEFORE the parent goal. The condition goal contains similar expression evaluation scheme as the calculation goal. However for a condition goal, the expression must evaluate either true or false. The result of the expression determines whether or not the parent goal is processed. If the expression is true example, group goal's condition goal can determine whether an entire section of questions is asked.

The Table 1 summarizes all the goal types. The abbreviations in the Reply Type column are $\mathrm{T}$ for Text, I for Index and $\mathrm{V}$ for Variable reply.

\begin{tabular}{|l|c|c|c|c|}
\hline Goal Type & User Interface & Child Goals & Process Order & Reply Type \\
\hline Text Entry & Yes & No & NA & T \\
\hline Numeric Entry & Yes & No & NA & T, V \\
\hline Single Select List & Yes & No & NA & T, I and/or V \\
\hline Multi Select List & Yes & No & NA & T, I and/or V \\
\hline Group & Optional & Yes & Before & None \\
\hline Info & Yes & Yes & After & None \\
\hline Calculation & No & Yes & After & T or V or I \\
\hline Condition & No & Yes & After & True/False \\
\hline
\end{tabular}

Table 1: Summary of the various goal types

To create a questionnaire the various combinations of goals must be put together in a hierarchical tree. When the questionnaire is played, the tree is interpreted in a depth first fashion. For an example, consider a small questionnaire that seeks to determine the height/weight ratio of a respondent. This requires the following information; the respondent's name height weight A calculation goal is also required to calculate the height/weight ratio. Figure 2 shows a possible tree structure for this. The dashed arrow height/weight ratio. Figure 2 shows a possible tree structur
shows how the tree is traversed in a depth-first fashion.

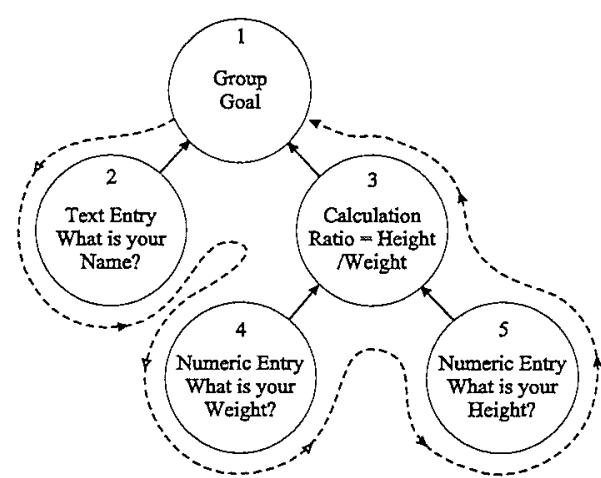

Figure 2: The tree structure of a simple questionnaire.

The following example demonstrales the use of a condition goal. Consider a questionnaire that asks a person's age. It will then display the age, but only if the person wants it to be shown. This requires two questions, a numeric entry goal for the age and a single select goal for the yes/no choice. An Info goal will display the age, but a condition goal attached to the info goal determines if the age will be shown. Figure 3 shows the tree structure of this example. There are several important points to note here,

1. Goal 2 is a sub goal of both goals 1 and 3. It is perfectly acceptable for a goal to be a sub goal of several different parent goals. The first time QuestEX processes goal 2, it will ask the question associated with goal 2. When QuestEX encounters goal 2 again, the information is already available. Thus the situation is a reference to goal 2. A referenced goal is shown in QuestED with a dotted border.

2. Goal 4 is a condition goal of goal 3. When QuestEX encounters goal 3, it will immediately check for a condition goal. On finding goal 4 , it will execute goal 4 and it's condition. To do this goal 4 must execute goal 5 . Once the result from goal 5 is available goal 4 evaluates the condition. If the condition is true, then goal 3 completes its processing by displaying its information. If the condition is goal 3 completes its processing by displaying its information. If the condition
false, theal 3 abandoned. A condition goal is shown with a heavy border.

Along with a goal's reply value, a goal also has a state. If a goal has not processed then it has no state. If a goal is successfully processed then its state is true otherwise its state is false. There are only two situations where the state of a goal can be set false. These are

1. By a Condition goal. If a parent goal's condition goal returns false, then the parent goal's state is set false.

2. In a Calculation goal. If a sub goal of calculation goal returns false then the calculation goal's state is set false. This is because the calculation goal no longer has all the information required to complete the expression. 


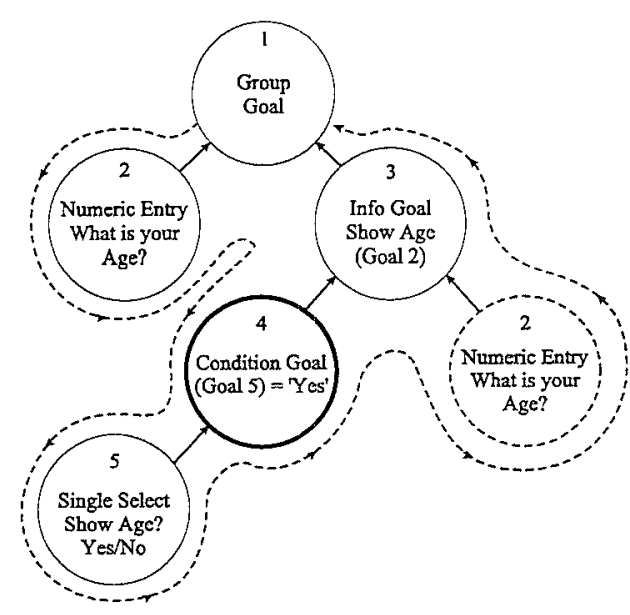

Figure 3: Info and Condition goal example.

The depth-first method of defining a questionnaires' structure is not immediately intuitive. The first version of Quest took a Flow Chart approach to defining the questionnaire structure. The flow chart approach is more obvious when defining the order and question flow aspects of a questionnaire. The hierarchy tree approach used in the current version of Quest approaches the questionnaire structure definition different. The questionnaire is seen as a number of information sources flowing from the respondent, through the processing elements of the questionnaire and out to summary form. The information gathered is regarded as more important than the actual ordering of the questions. By focusing on the flow of information rather than the flow of questions, Quest is able to make a more consistent approach to the whole questionnaire process.

The user interface of a questionnaire is defined by a series of templates. A template is a description of how the screen should appear for a particular goal type. Each a desciption of how the sceen shold apear for a paricular go $A$ tempe Each template consists of a number of objects. Some of the template objects are simple graphic primitives such as lines, circles and rectangles. Others are just place holders, for example the Question Text object defines where a goal's actual question text is drawn on the screen. A number of goals may share a template, or individual goals may have their own templates. New templates may be created and modified as needed.

A number of Summaries may be defined for each questionnaire. Each summary A numsists of a number of summary entries which map to the goals in the questionnite structure. Each entry is a piece of text plus a goal reference. When the summary is processed, each entries' text is formatted with the goal reply and is witten out to file. The entry text and the formatting is completely author-definable, so custom summaries can be easily created for different purposes.

\section{The Questionnaire Editor.}

\subsection{Installing QUEST.}

QUEST is distributed via floppy disk. To install QUEST insert the floppy disk labeled 'QUEST -- Disk \#1' into a floppy disk drive. Then run the setup program on the floppy disk, this can be done by,

1. Double clicking SETUP.EXE in file manager or Explorer.

Start file manager, and change to the floppy disk drive that contains the QUEST files. Use the mouse to double click on the SETUP. EXE file item, or select the SETUP. EXE file item with the arrow keys and TAB and press ENTER.

2. Using the Run command in either file manager or Explorer.

Select the Run command from either file manager's or program manager's File menu. Type in the full path for the SETUP. EXE file and press the Enter key or the OK button.

Now follow any prompts that are displayed during installation.

\subsection{Running QuestED.}

QuestED can be started in a number of different ways,

1. By double clicking on a program manager icon or shortcut. This is the recommended way of starting QuestED. The icon or shortcut should have been created during installation.

2. By double clicking QUESTED.EXE in File Manager or Explorer

To start QuestEX this way, run file manager or Explorer and change to the directory containing QuestEX. Use the mouse to double click on the OUESTED.EXE file item, or select the QUESTED. EXE file item with the arrow keys and TAB and press ENTER

3. By using the Run command.

Select the Run command from either file manager's or program manager's File menu or the Start Menu. Type in the full path for the QUESTED.EXE file and press the Enter key or the OK button.

4. By file association

During installation a file association is setup for the extension .QS2 and the program as QUESTEX.EXE. This can be changed so that association is with QUESTEX. EXE. Then QuestEX can be started by double clicking on the QS2 data file in file manager. Note that an association can only be set-up for QuestEX or QuestED, not both. 
5. With a command line argument.

When using the above methods 1 and 3, a path and filename for a.QS2 questionnaire file can be specified after the QuestED executable filename This will automatically load the questionnaire file once arted. For example, the fo QuestED and load the questionnaire file HEALTH. OS2.

$C: \backslash Q U E S T \backslash Q U E S T E D$. EXE C: \QUEST \HEALTH.QS2

Once started QuestED will appear as in Figure 4.

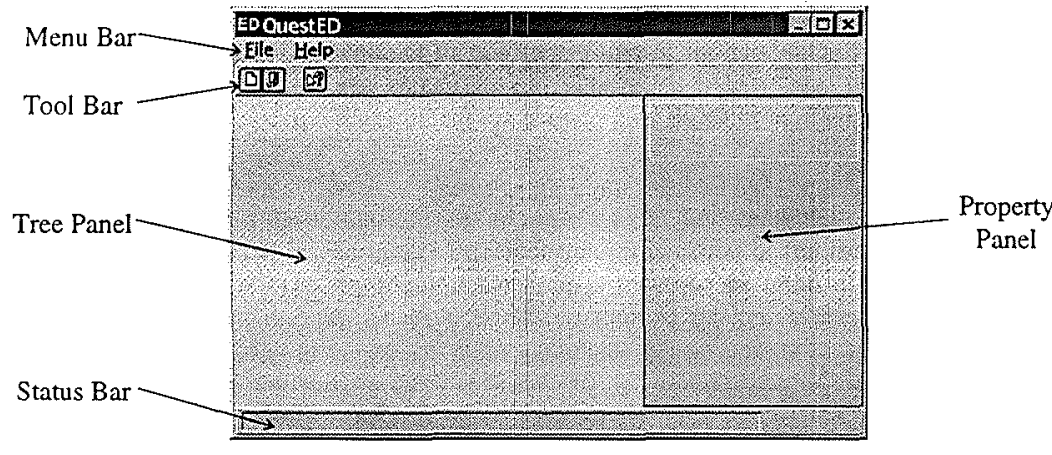

Figure 4: QuestED's initial appearance.

\subsection{File Operations}

Initially there are three options in the file menu. These are

1. New. Creates a new questionnaire within QuestED.

2. Open. Opens an existing questionnaire file into QuestED.

3. Exit. Closes QuestED immediately.

Once a questionnaire has been either created or opened into QuestED, the file menu changes. In addition to the three options above, there are two more options,

1. Save. Saves the current questionnaire file. If no filename has been given then the standard Save File dialog box prompts the user to enter a filename.

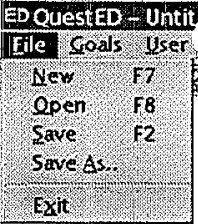

2. Save As. The standard Save File dialog box prompts the user to enter a filename. The current questionnaire is then saved under this filename.

\subsection{The Tree Panel}

Once a questionnaire has been loaded in QuestED, the Tree Panel will appear as in Figure 5 .

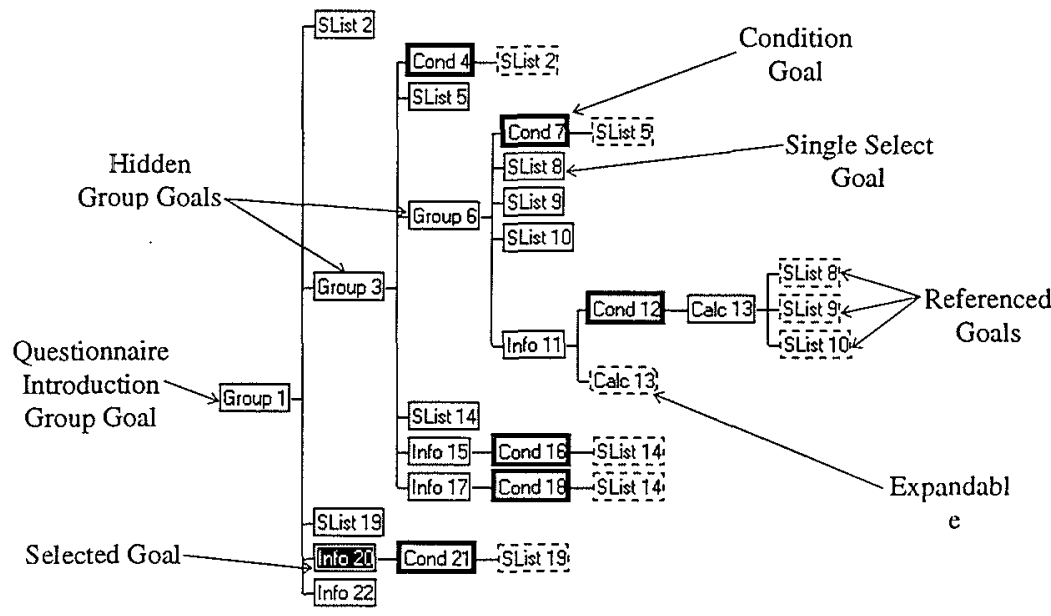

Figure 5: Tree panel view.

The important points in this figure are,

1. Selected Goal. The currently selected goal appears inverted.

2. Goal Notation. Each goal is labeled with a short goal type identifier and the goal number identifier. The Goal type identifiers are given in Table 2.

3. Questionnaire Introduction Group Goal. Goal number 1 is always defined as the Questionnaire Introduction goal. This is the first screen the respondent sees when Qhe questionnaire starts.

4. Hidden Group goals. Goal 3 and 6 simply 'group' their sub goals together so that they appear as one goal. Both these goals are hidden to the respondent as they have no user interface.

5. Condition Goal. All the condition goals have a thick border to differentiate from other sub goals.

6. Referenced Goals. The referenced goals have a dotted border to indicate that they are not the first instance of the goal. 
7. Expandable Goal. It is possible to collapse the children of a goal by double clicking the left mouse button on it. The collapsed goal then appears with a rounded border. Double click on the goal to expand the hidden sub goals.

\begin{tabular}{c|c|c|c} 
Goal type & Label & Goal type & Label \\
\hline Group Goal & Group & Text Entry & Text \\
\hline Info Goal & Info & Numeric Entry & Num \\
\hline Calculation Goal & Calc & Single Select List & Slist \\
\hline Condition Goal & Cond & Multi Select List & MList
\end{tabular}

Table 2: Goal type abbrevaitions.

\subsection{The Property Panel}

The Property Panel is located to the left of the Tree Panel. Its purpose is to show the properties of the currently selected goal in the tree panel. The properties of the selected goal can be directly modified in the property panel. For the different types of goal, there is a different property panel, but they all share features. There are four different property groups that may appear on each panel. These are,

1. Goal Information. Gives basic information about a goal. For example, the goal identifier, goal type, condition goal, description, title and sub goals. The description differs for each of the goal types. That is a calculation goal has calculation, a condition goal has a condition, etc. The Question goal types do not have the sub goal field. Figure 6 shows the Group and Text Entry group information panels.
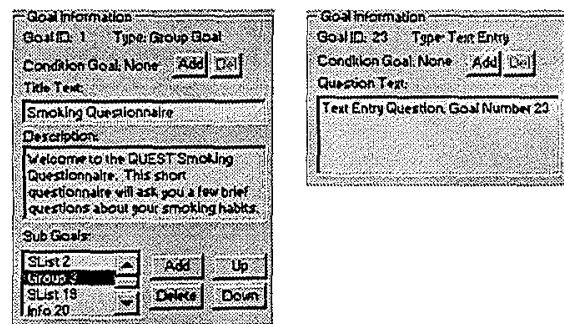

Figure 6: Two of the Goal Information panel.

Reply Field. Only Question goals have this group. This group sets the options for the question's reply field. For example a single select list question's pre-defined choices are entered here. Figure 7 shows the Text entry and single select list reply fields.

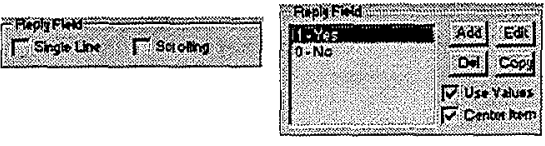

Figure 7: The Text Entry and Single Select List Reply Fields:

Option field. This group contains a series of options relating mainly to that goal type. Figure 8 shows the standard option field and the numeric entry option field.

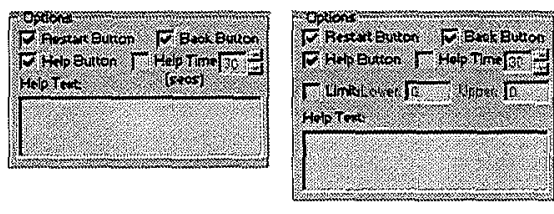

Figure 8: The two option fields.

User Interface. This group only applies for those goals that are allowed to display a user interface. This is all goals excluding the condition and calculation types. Figure 9 shows the user interface field.

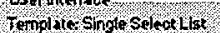

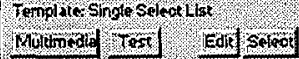

Figure 9: The User interface field.

Table 3 summarizes the various combinations of the above four fields on each property panel. In the Goal Info column, Simple means that just the basic fields are in the Goal Information group. Sub goals means that a list of sub goals and sub goal manipulation controls are also present.

\begin{tabular}{|l|c|c|c|c|}
\hline Goal Type & Goal Info & Reply Field & Options Field & User Interface \\
\hline Group & Sub goals & No & Yes & Yes \\
\hline Info & Sub goals & No & Yes & Yes \\
\hline Calculation & Sub goals & No & No & No \\
\hline Condition & Sub goals & No & No & No \\
\hline Text Entry & Simple & Yes & Yes & Yes \\
\hline Numeric Entry & Simple & Yes & Yes & Yes \\
\hline Single Select List & Simple & Yes & Yes & Yes \\
\hline Multi Select List & Simple & Yes & Yes & Yes \\
\hline
\end{tabular}

Table 3: Summary of the Property Panel for each goal type. 


\subsection{Goal Operations}

There are a number of operations that can be performed on a goal. Some of these operations apply to eerlain goal types only while others apply to all goals. All these operations can be invoked through the Goals menu or from the Properties Pancl. Each of the operations are described below,

- Add Condition. This operation adds a condition - Add Condivion. This operation adds a condi sub goar to the curnen goul. This is the only way Add condition Ctilta add a condition goal. This operation is available Delete Condilion ctrit b. for all goal types, unless the current goal already has a condition goal.

- Delete Condition. This operation removes the condition sub goal of the current goal. It will also delete any sub goals of the condition goal. A dialog box will be displayed asking for confirmation of the delete. See also the entry 'Delete Sub Goal'. This operation is only available when the current goal has a condition goal.

The next four operations only apply to the Control Goals. All the control goals can have sub goals. These operations manipulate the sub goals of the current goal.

- Add Sub Goal. This operation adds a sub goal to the current goal's sub goal list. The dialog box in Figure 10 will appear. This dialog box gives the choice of adding a new goal or adding

a reference to an existing goal. Using the radio

\section{Add sub God}

geteres of 60.81

Move sut (xiol thp: N100e sut Gat id os

button will enable the drop down list associated with each choice. When adding a new goal, the goal type of the new goal can be chosen from the drop down When referencing an existing goal the drop down list contains a list of all the goals in the questionnaire.

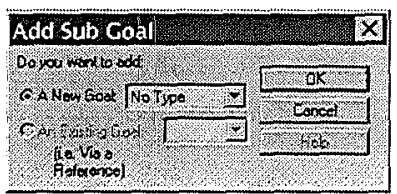

Figure 10: The Add Sub Gral dialog box

- Delete Sub Goal. This operation is used to delete a goal from the questionnaire. To use it, a sub goal must first be selected from the Sub Goal list on the Property Panel A dialog box will be displayed asking for confirmation of the delete. It is

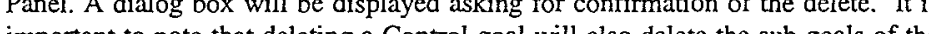
control goal in a recursive fashion. Three rules however govern what is deleted,

1. If the goal is unique, that is, it is not referenced by any other goal then it will be deleted.
2. If the goal is not unique and the other referenced instance of the goal is also down the branch being deleted then both goals are deleted.

3. If the goal is not unique and the other referenced instance of the goal is not down the branch being deleted then goal is deleted, but the referenced goal down the

This simply ensures that branches are correctly maintained. For example in Figure 5 if 'Group 6 ' was selected as the current goal. We could then delete the sub goal 'Info 11'. This would delete both the original and referenced instances of 'Calc 13'. However only the referenced instances of goal 8,9 and 10 would be deleted. The original goals 8,9 and 10 would still exist. If a summary entry has been defined for the goal being deleted, then that summary entry will be deleted as well. For more information on summaries see section 3 .

- Move Sub Goal Up. This operation is used to move a sub goal forward in the questionnaire order. Thus it is possible to arrange the order of goal within a group of goals.

- Move Sub Goal Down. This operation is used to move a sub goal back in the questionnaire order. It does the opposite of the Move Sub Goal Up operation.

For example in Figure 5 if 'Group 6' was selected as the current goal. We could then reorder the goals 8,9 and 10 by moving them forward and back using the Move Sub Goal operations.

The Calculation, Info and Condition goal types all have an Insert Sub Goal Reference operation. This is because the sub goals of the goal types contribute to the functionality of the goal type. For example the sub goals of the Calculation goal are used as variables in the calculation's expression. The Condition goal uses its sub goals in a similar fashion. The Info can embed its sub goal replies in its user interface text.
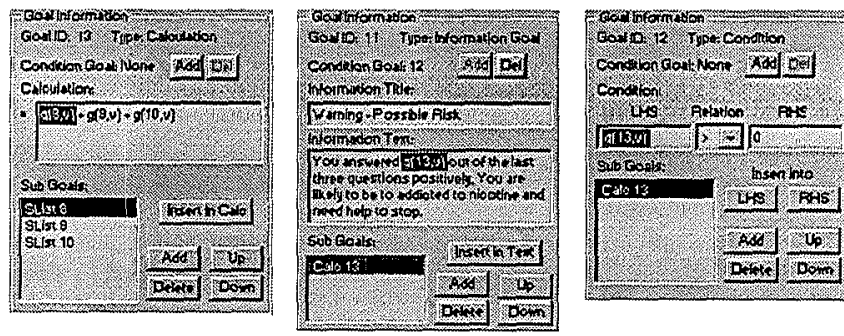

Figure 11: The three goal types with the Insert Reference Operation

The Calculation and Info goal types are similar as the reference can be inserted in one place. That is either in the calculation or the information text. The Condition is different as each condition consists of three parts, the left hand side, the relation and 
the right hand side. A goal reference may be insert in either the left hand side or the right hand side of the expression.

To insert a reference first select the place in the goal's text where you want the reference to appear. Then select the goal you want to reference from the sub goal list Press the Insert button for that Property Panel. The dialog box in Figure 12 is displayed. Choose from the drop down list how you want the reference displayed. This is either as a Text string, integer Index or a real Variable. A reference such as $\mathrm{g}(13, \mathrm{i})$ will be created and inserted into the goal's text. The ' $\mathrm{g}$ ' indicates a goal reference, the ' 13 ' is the sub goal being referenced, and the ' $i$ ' is the variable type. The variable type can be either ' $i$ ' for index, ' $s$ ' for string or ' $v$ ' for variable.

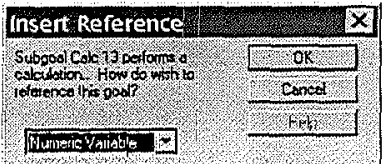

Figure 12: An example of the Insert Reference dialog box

Note that there is difference between a 'Goal Reference' and a 'Reference Goal'. 'Goal References' only exist in the context of the above three goal type's functionality. A 'Reference Goal' is the second instance of a particular goal. That is a goal with a dotted border in the tree panel.

\subsection{Reply Field Operations}

The Reply Field operations can only be performed on the Question goal types. Figure 13 shows the reply field for the Single Select List and the Multi Select List goal types. The list box contains the pre-defined replies

Add Beply.
Edit Rejply
that are presented to the respondent in the question. Each item in the list box shows the text for the reply and if the 'Use Values' check box is checked, the value associated with that item. If 'Use Values' is unchecked, then only the reply text is shown in the list box. 'Use Values' is important as it allows a numeric variable to be associated with each item.

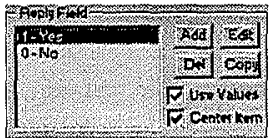

Figure 13: The Reply field for the Two List based Question types

The 'Center Item' check box allows all the replies to be displayed centered in the list when presented to the respondent. Otherwise the replies are displayed left justified.

The four buttons in the Reply Field allow for the direct manipulation of the responses.

- Add Reply. This operation displays the dialog box in Figure 14. If 'Use Values' is unchecked, then the Value field is not displayed in this dialog box. Here the attributes for the new reply can be entered.

- Edit Reply. This operation is available when a reply has been selected from the reply list box. It displays the dialog box in Figure 14 but it contains the attributes from the selected reply.

- Delete Reply. This operation is available when a reply has been selected from the reply list box. It will delete the selected reply.

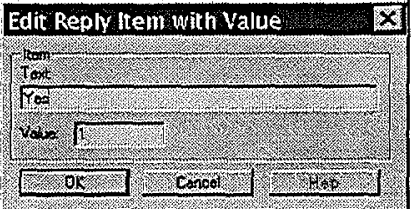

Figure 14: The edit reply dialog box

- Copy Replies. This operation allows the replies from one list based goal to be copied to other list based goals. The dialog box in Figure 15 appears. All the list based questions in the current questionnaire appear in the multi-select list box. 
Any number of questions can be selected here. After closing the dialog box, the replies in the currently selected goal will be copied to the questions selected in the dialog box. For example there maybe a large number of similar list based questions in a questionnaire. First all the list based questions would be created in the questionnaire. The replies would then be defined for the first list based question. The Copy Replies operation would then be used to copy these replies to all the other questions in the questionnaire.

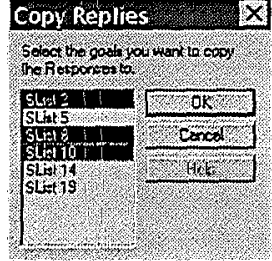

Figure 15: The Copy Replies dialog box

Figure 16 shows the Reply Field for the Text Entry and the Numeric Entry question goals. By default the edit field for these two questions is multi line and non scrolling. This means when the respondent reaches the end of the first line of text, the text cursor in the edit field will move to the start of the next line in the edit field. Once the cursor in the edit field will move to the start of the next line in the edit field. Once the
respondent has filled the edit field with text, then no more text can be entered. However by checking the 'Single Line' check box, the edit field will be limited to a single line. Checking the 'Scrolling' check box will allow the edit field to scrolling once the respondent has filled up the field.

\section{rstogle int r. Ts scouling.}

Figure 16: The Reply Field for the Text based Question.s.

\subsection{User Interface Operations}

The User Interface operations affect how the current goal will appear when the questionnaire is played. Each goal's user interface is based on a template. A template is a description of how the screen will appear. A number of goals may share a template and consequently they will all appear similar. Each template is defined for a particular goal type as the different goal types require different user interface. For example a Text Entry template contains an edit field whereas a Single Select List template has a list box instead. For convenience a set of default templates have been provided. For a better understanding of the templates see section 4 .

While the templates make it possible to define the minimum number of user interfaces for a questionnaire, it is possible to further alter the appearance of each goal. Each goal that has a user interface has a set of options. These options control how the the goal's template is displayed. Figure 8 shows the two option fields for all the goals.

- Restart Button. If this is checked then the Restart or 'Quit' button will appear in the template. Pressing this button ends the current questionnaire session.

- Back Button. If this is checked then the 'Back' button will appear in the template. Pressing this button will take the respondent back to the last question answered.

- Help Text. The text that is entered in this field is displayed in the templates Help text field.

- Help Button. If this is checked then the 'Help' button will appear in the template The Help Text will remain hidden until the Help Button is pressed. If Help Button is unchecked then the Help Text will display immediately.

- Help Timer. If this is checked then the Help Text will be hidden until the specified time interval has elapsed. Once elapsed the help text is displayed in the Help Text field. The time interval can be changed via the spin control.

- Limits. This only applies for the Numeric Entry goal type. If this is checked, then a lower and upper limit can be set for the goal. The respondent must enter a number within these limits. This is useful in stopping the respondent entering number within these

By default each goal uses the default template for its type. To customize the goals setting the following operations are used.

- View Templates. This operation displays the Template Manager. This is shown in Figure 28. The Template Manager is discussed further in section 4 . 
- Select Template. This operation displays the list of templates that match the current goal's type. The desired template can be chosen from this list.

- Edit Template. Choosing this option starts the Template Editor for the current goal. For more information sece section 4

- Test Goal. Choosing this operation gives a preview of how the goal will appear when the questionnaire is played. To close the preview press the 'OK' Button.

- Multimedia. This operation displays the Multimedia options dialog box for the current goal. This is shown in Figure 17. It is further discussed in the next section.

\subsection{Multimedia Options.}

Figure 17 shows the Multimedia dialog box. Each goal that has a user interface has a sut of associaled multimedia options. There are four multimedia groups in the dialog box, Digital Audio, Video Animation, Music and Graphic Images.

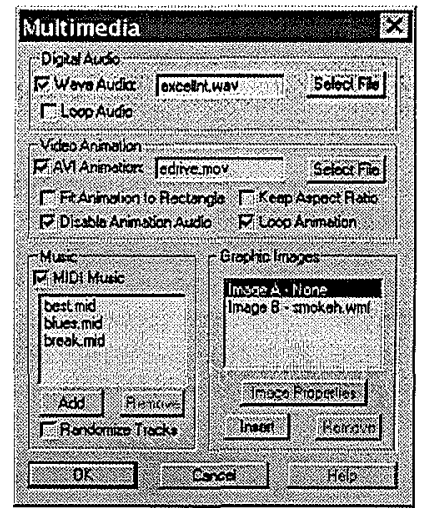

Figure 17: The Multimedia options dialog box.

Digital Audio. Checking 'Wave Audio' control enables the digital audio option for this goal. Pressing the 'Select File' button displays a standard open file dialog box. Use this dialog box to choose a Wave file (*.WAV). Note that the Wave file should be in the same directory as the main questionnaire file. Checking the 'Loop Audio' control makes the audio clip repeat continuously.

Video Animation. Checking the 'AVI Animation' control enables the video animation option for this goal. Note that it will also disable the 'Digital Audio' option as the Video Animation and the Digital Audio must share a single sound channel. Use the 'Select File' button to choose an animation file (*.AVI). If the current goal's template contains an animation rectangle then the animation file will be played in this rectangle. Checking the 'Fit Animation to Rectangle' will stretch the animation to the animation rectangle. Otherwise the Animation will play centered in the animation animation rectangle. Otherwise the Animation will play centered in the animation
rectangle. Checking the 'Keep Aspect Ratio' control forces the animation to play with its pre-defined aspect ratio even when stretched. Checking the 'Disable Animation Audio' disables the animation's audio channel and re-enables the Digital Audio's audio channel. Checking the 'Loop Animation' control makes the animation repeat continuously. With the correct MCI drivers it is also possible to play Apple Quicktime Movie files (*.MOV) and MPEG files (*.MPG).

Music. Checking the 'Midi Music' controls enables the music option for this goal. Pressing the 'Add' button displays an extended select open file dialog box. Use this dialog box to select any number of MIDI music sequence files (*.MID). The selected music files will be added to the play list. The 'Remove' button is used to remove any 
selected MIDI file from the play list. For all goals except the Group goal, the files on the play list are played one at a time. When the respondent presses the 'OK' button the music is stopped. With a Group goal, the music starts when the group goal is displayed. The play list stops when all the group goal's sub goals have been processed or another goal is processed that has its Music option enabled. Thus a play list for tho entire questionnaire can be defined in the questionnaire introduction goal. This play list will play continuously for the duration of the questionnaire. Checking the 'Randomize Tracks' control mixes the play list randomly so the play list will be 'Randomize Track'

Graphic Images. Like the Video Animation, this option requires that Image rectangles are defined in the goal's template. Each Image rectangle in the template is initially listed in the image list as 'Image A -- None'. 'Image A' identifies the image rectangle as it appears in the Template Editor. 'None' indictes that a file has not rectangle as it anears in as 'Inage $A$. No been assigned for his image. To all press the 'Insert button. This will display an open fle dialog box. Choose a file mage from one of the allowed types, Windows Bitmap (*.BMP), Zsoft PaintBrush (*.PCX) and Windows Metafile (*.WMF). To deselect an image select it from the image list and press the 'Remove' button. To change the image properties, select the image from the list and press the 'Image Properties' button. This will display a dialog box containing a 'Stretch Image' control. Checking this control causes the image to stretched the image rectangle as defined in the template editor.

\subsection{Questionnaire Operations}

The Questionnaire operations effect how questionnaire works as a whole. There are currently threc questionnaire operations, Question aire options, Renumber questionnair and Run QuestEX.

Choosing the Questionnaire Options operation displays the dialog box in Figure 18a. There are three fields within this dialog box, all relating to the operation of the questionnaire,

- Exit Password. By checking the 'Enable Password' control, a password can be entered in the Password edit control. When the 'Quit' button is pressed from the Questionnaire Introduction screen the dialog box in Figure 19. The correct password must be entered before QuestEX will close. This is a useful way of stopping the respondent from exiting QuestEX

- Enable Log File. As QuestEX is running, it can produce a log file of the operations during its execution. This can be a useful source of information when a operations during its execution. This can be a useful source of information when a
questionnaire is behaving unexpectedly. It is recommended that the $\log$ file be questionnaire is behaving unexpectedly. It is recommended that the log file be
disabled once the questionnaire is working correctly. This is because the log file disabled once the questionnaire is working correctly. This is because the log file
grows each time the questionnaire is played. Thus it can build up to a rather large

- Security Mode. To further enhance the protection that the Exit Password gives a questionnaire the security modes can be enabled. There are currently two modes. The 'Testing Mode' allows the questionnire to run as a nom Wins Tpplication would It is poss application would. It is possible to task switch away from QuestEX while it is playing a questionnaire. Task switching is accomplished by either using the AltTab or Ctrl-Esc key combinations. The 'Safe Mode' disables task switching while QuestEX is running. Therefore the only way to exit QuestEX is by pressing the 'Quit' button on the Questionnaire introduction screen. Unfortunately the respondent can still press the Ctrl-Alt-Del key combination. This is quite serious as the respondent can close Windows in this situation. There are three possible ways of dealing with this problem,

1. Physically disabling the 'Delete' key. On many keyboards it is possible to remove the keys. This is done by inserting a screwdriver under the key and gently levering it off. This could indeed be done for all the 'Delete' keys' neighbors. The hole in the keyboard could then be covered with card board and tape.

2. Configuring QuestEX to be the Windows Shell program. This option would load QuestEX as the system shell instead of Program Manager or Explorer. Thus it would be no longer possible to run other Windows programs in the same session. QuestEX would be the only program running. The benefit of 
this is if the computer boots straight into Windows then it will automatically run QuestEX. So if a respondent keeps on rebooting the computer QuestEX will always automatically start. This is a short term goal for the Quest system.

3. A Window Virtual device driver for the keyboard could be written that disables the Ctrl-Alt-Del key combination at an operating system level. This is a long term goal for the Quest system and is the best solution for this problem.
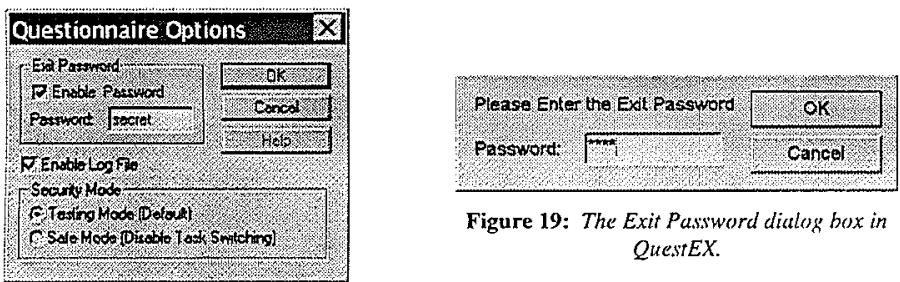

Figure 19: The Exit Password dialog box in QuestEX

Figure 18: The Questionnaire Options dialog box.

The Renumber Questionnaire operation scans the questionnaire and renumbers i according to the order that QuestEX processes the goals. This is a useful operation for a number of reasons.

1. It shows the order in which the goals are processes and consequently the order in which the questions are asked.

2. It can clean up the goal numbering especially after a lot of editing.

3. It can force the Tree Panel to correctly display the questionnaire tree Occasionally the Tree Panel may not expand a goal correctly. This may occur if a collapsed goal is moved forward in the questionnaire order. Using the Renumber Questionnaire will expand the questionnaire structure correctly.

The Run QuestEX operation starts QuestEX as if you had just run it from Program Manager or the Explorer. You can then start any questionnaire as you would normally. Note to run the current questionnaire in QuestED, it must be manually saved before running QuestEX.

\subsection{Editor Options.}

The Editor Options govern how QuestED displays the Tree Panel and the Property panel. Selecting the Editor Options menu item will display the dialog box in Figure 20. There are three main editor settings that can be changed by this dialog box.

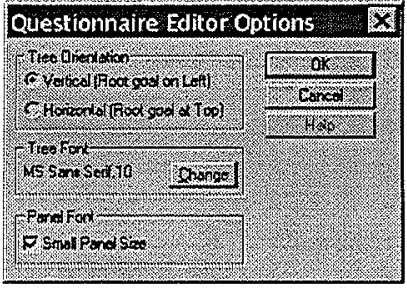

Figure 20: The Editor Options dialog box

The Tree Orientation group affects how the Tree Panel displays the questionnaire tree. The Vertical option displays the Questionnaire Introduction goal on the left side of the panel. The sub goals are then processed from top to bottom and each level of the hierarchy expands to the right. The Horizontal option displays the Questionnaire Introduction goal at the top of the panel. The sub goals are processed from left to right and the levels expand top to bottom. See Figure 21 for an example of the two options.

The Tree Font setting can be used to change the appearance and size of the tree font The tree font is used in the label for each leaf in the questionnaire tree. By increasing the size of the font, the size of each leaf is also increased.

The Panel Font setting can be used to change the size of the of Property Panel. When using the low resolution of 640 by 480 pixels the bottom portions of many of the property panels may be obscured. Checking the 'Small Panel Size' control makes each panel use a smaller font for the panels. This should ensure that all the panels are completely visible.
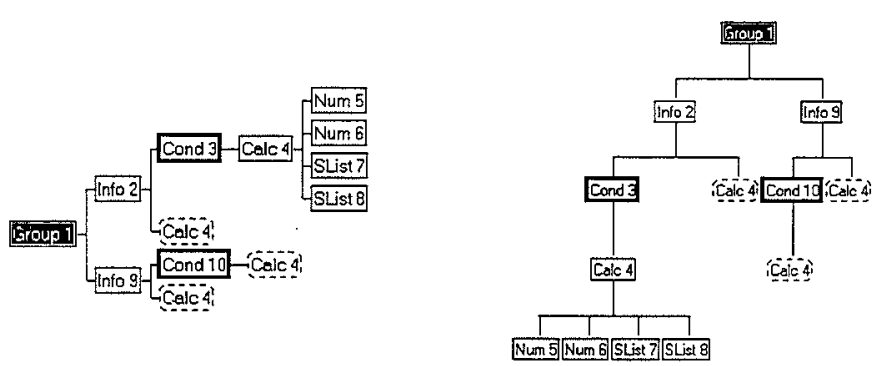

Figure 21: The two possible tree panel orientations, Horizontal and Vertical. 


\section{The Summary Editor}

The summary editor allows an author to define a series of 'reports' for each respondent that answers a questionnaire. Each report is called a 'Summary'. Each summary can be saved in an ASCII text file and can also be printed to a printer. For each questionnaire, any number of summaries can be defined. The point of this is that one summary can be defined for the author's records, one for the respondent and so on.

Each summary consists of a series of summary entrics. Each summary entry corresponds to a goal in the questionnaire. However not every goal in the questionnaire needs to be included in the summary. Each summary entry is similar to an Info goal. It consists of three parts,

1. A goal reference. This defines the 'type' of the goal reply used in the summary entry. This is either the Text string, Integer index or Numeric Variable.

2. A text string. This text string is what will be displayed in the summary. By default it is the same as the questionnaire text but does not have to be. The most important part of the text string is the ' $\wedge$ ' symbol. This is where the goal reference is inserted. So in order to actually see what the respondent entered into the questionnaire, this symbol must be somewhere in the text string.

3. A formatting delimiter. This defines what follows the summary entry. It is used to provide simple formatting options for the summary. The following delimiters may be used

- None. No formatting, so the next summary entry will immediately follow the current entry. Useful creating a paragraph of text out of a number of summary entrics.

- New Line. Inserts a new line after the current summary entry. The next summary entry will start on the next line.

- Blank Line. Inserts a blank line after the current summary entry. The next entry will start after skipping a line. This is useful creating headings out of group goal entries.

- Tab. Inserts a tab stop after the current entry. Tab stops occur every eight characters. This is useful for lining up entries into columns. It is also useful for creating summaries that can be imported in to spreadsheet and database applications. Most spreadsheet and database applications can import tab separated and comma separated text files.

- $1 / 2$ Page Tab. Inserts a half-page tab stop. This is useful for lining entries in two columns across the page.

- Centered Line. Centers the current entry across the line. Useful making headings.

- Right Line. Aligns the current entry to the right hand side of the summary.

Summaries are created from the Summary Manager. Choose the 'View Summaries' menu option to display the Summary Manager dialog box. See Figure 22.

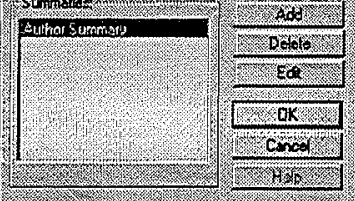

Figure 22: The Summary Manager dialog box

Press the 'Add' button to create a new summary. The Summary Style dialog box will appear, see Figure 23. Choose the appropriate choice and press the 'OK' button. The Person Readable style gives the full range of formatting delimiters and the default summary entries are based on the associated goals question text. The Computer Readable style limits the range of formatting delimiters to those more suited to
importing into other applications. The default summary entries are simply based on importing into other application.

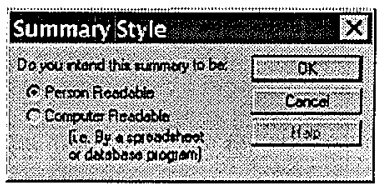

Figure 23: The Summay Style dialog hox

Once the choice has been made the Summary Edit dialog box appears. See Figure 24. The Summary Options group has three main controls. The Summary Name field is where the name of the summary is entered. If the summary is to be saved to disk, then check the 'Output to File' control. Likewise check the 'Output to Printer' if the summary is to be printed.

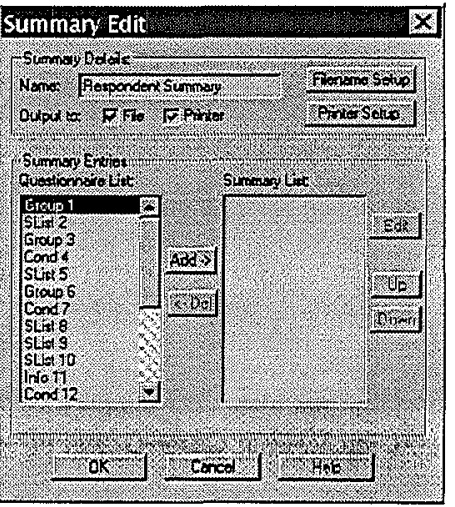

Figure 24: The Summary Edit dialog box. 
Once one of the 'Output to' options have been enabled, that option can be further customized with 'Filename Setup' and 'Printer Setup' button. Figure 25 shows both the 'File Setup' and 'Print Setup' dialog boxes. The Directory field in the 'File Setup' dialog specifies a directory name that is created below the questionnaire directory The Filename field is combined with the number of the respondent to create filename. The summary is then saved using this filename. The Printer font used while printing the summary can be changed in the 'Print Setup' dialog box by pressing the 'Change' button. Any font maybe chosen, but fixed pitch fonts such as Courier produces the best output. When using a True Type font the size of the matched to the size of the printer page by checking the 'Stretch to Page' control.
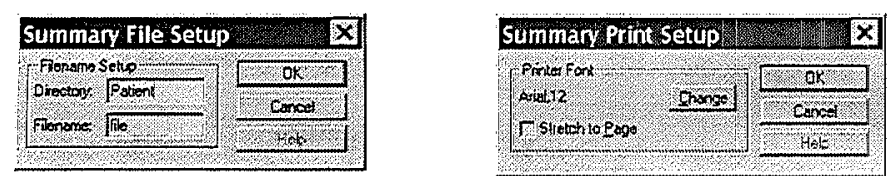

Figure 25: The Summary 'File Setup' and 'Print Setup' dialog boxes.

In the Summary Entries group are the 'Questionnaire List' and the 'Summary List'. Each item in the 'Questionnaire List' is a goal from the current questionnaire. Selecting a number of items from this list box and pressing the 'Add $\rightarrow$ ' button transfers the selected items to the 'Summary List'. Each item that appears in the transfers the selected items to the 'Summary List'. Each item that appears in the
summary list will be used in generating the actual summary. Items maybe transferred summary list will be used in generating the actual summary. Items maybe transferred back to the 'Question
the '<- Del' button.

Three operations can be performed on the summary entries in the 'Summary List'. The entries can be moved up and down in the summary order by selecting them and pressing either the 'Up' and 'Down' buttons. Once selected an entry can be edited by pressing the ' $\mathrm{Edit}$ ' button or by double clicking on it. This displays the dialog box in Figure 26. The three fields in this dialog box correspond to the three parts of the summary entry discussed earlier.

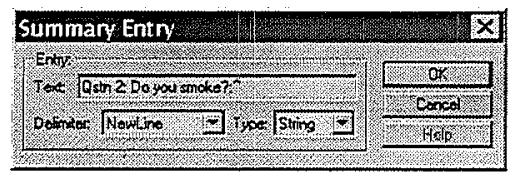

Figure 26: The summary entry dialog box.

Once the all the required summary entries have been defined, exit from the Summary Edit dialog by pressing the 'OK' button. Any changes to the summary can be discarded by pressing 'Cancel'. In the Summary View dialog box a summary can be deleted from the questionnaire by selecting the summary from the list and pressing the 'Delete' button. An existing summary can be edited by pressing the 'Edit' Button. This will display the Summary Edit dialog box again.

\section{The Template Editor}

The Template Editor is used to create a customized user interface for a questionnaire. A template is a description of how the screen should appear for a particular goal type One template can be used for all goals of a particular type or each goal can have it own template. The objects within a template describe where the object should be positioned and how it should appear. The actual content for the object is defined by the goal. For example, all question templates contain a question text object. The question text object is used to position and format each goal's actual question text An animation object provides the positioning for the animation file defined in the goal's multimedia properties.

A set of default templates has been provided as a starting place for your user interface. Each of these templates can be modified to suit, and new templates can be created based upon these default templates. For example the Questionnaire Introduction goal uses the Default Group Goal template. However a new template may be based on this style and then modified to suit the Questionnaire Introduction better.

\subsection{Template Operations.}

The simplest operation template operation for a goal is to select a template. Choosing the 'Select Template' menu item or pressing the 'Select' button on the Property Panel will display the Select Template dialog box. See Figure 27. Only the templates that match the current goal's type appear in the list. The 'None' template option is only available if the current goal is a group goal. Choose the desired template and press the 'OK' button.

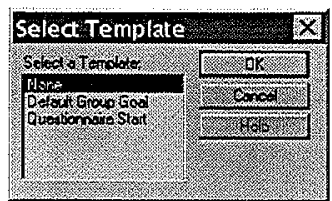

Figure 27: The Template Select dialog box.

To view all the templates in the current questionnaire choose the 'View Templates' menu option. This displays the 'View Templates' dialog box, as shown in Figure 28. This dialog box is effectively the template manager. There are five main operations that can be invoked from here.

- Select. If enabled, this function selects the template for the currently selected goal.

- Edit. Selecting this option opens the template editor with the selected template. See Section 4.2. 


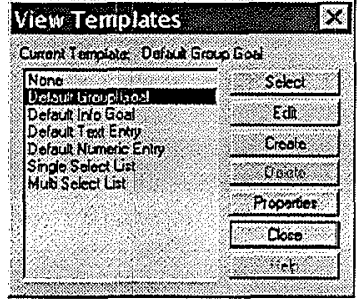

Figure 28: The Template Manager dialog box.

- Create. A new template can be created based on another template with this operation. This allows the creation of a customized template that is used as the base for all the other templates in the questionnaire. For example, the default Group Goal template maybe customized with a series of images and graphic objects. This New Group Goal template can then be used as the base for each of the other goal types. The customized objects in the New Group Goal template are transferred to the new derived templates. To create a new Text Entry template, select the base template from the Template list in the 'View Templates' dialog box and pressing the 'Create' button. The New Template dialog box in Figure 29 will appear. Enter the new templates' name in the Name edit field and choose the appear. Enter the new templates' name in the Name edit field and choose the
template target type from the drop down list. In the example this will be 'Text template target type from the drop down list. In the example this will be 'Text
Entry'. Press the 'OK' button. The new template will appear in the template list Entry'. Press the 'OK' button. The new template will appear in the template list
of the View Template dialog box. The Create operation is able to convert to and from all the different goal types if so needed. For example a Single Select list template can be created from and Info Goal template, and so on.

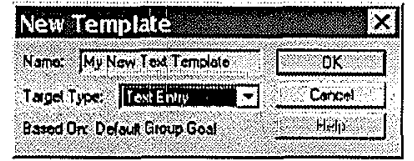

Figure 29: The New Template dialog box.

- Delete. This operation will delete the currently selected template from the Template list. A confirmation message box will appear before the template is deleted.

- Properties. This operation displays the Template Properties dialog box as in Figure 30. The default properties of each template can be changed here. The fields are,

1. Name. The name of the template can be changed in this field

2. Help Message. This is the default help message that is displayed when the respondent performs an inappropriate action. For example the respondent presses the 'OK' button in a Text Entry question without entering a reply.
3. Display Mode. The display mode controls whether the template is drawn as a metafile picture or a bitmap picture. In general the metafile option is drawn faster, but slows as the number of template objects increases. The bitmap option always is drawn at the same speed, but is slower than the metafile option for simple templates. The bitmap option is chosen by default when the template contains one or more graphic images.

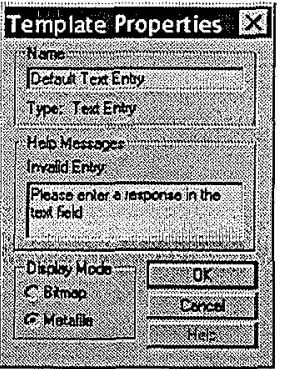

Figure 30: The Template Properties dialog box.

The current goal and its template can be previewed by choosing the 'Test Goal' menu item or pressing the 'Test' button on the Property panel. This displays the goal's user interface exactly as it would appear in QuestEX. The multimedia properties of the current goal are also played. To exit from the preview mode, press the template's 'OK' button.

\subsection{The Template Editor.}

The Template Editor is a graphical template object editor. The Property and Trec Panels are hidden, and the Template View appears. The Template View contains a simple graphical object representation of the template and a floating dialog box. The floating dialog box contains the template object list and a series of buttons. Figure 31 shows a view of the Template Editor. The important points in this figure are,

- Template Title. The name of the template appears in the title bar of QuestED.

- Template Objects. Most of the template objects appear in the Template view as white rectangles with a text label.

Template Background. This shows the boundaries of the template in the Template View.

- Template Object List Dialog. This dialog box contains a list of all the objects in the template. The order the objects appear in the list directly relates to which objects appear in front of other objects. For instance the background is always first on the list, so it appears underneath all other objects. The button objects all appear at the end of the list, and appear in front of the other objects. 
- Selected Items. The items selected in the Template list appear bounded by the Selection Rectangle in the Object View.

- Selection Rectangle. This rectangle appears around the selected objects in the Template View.

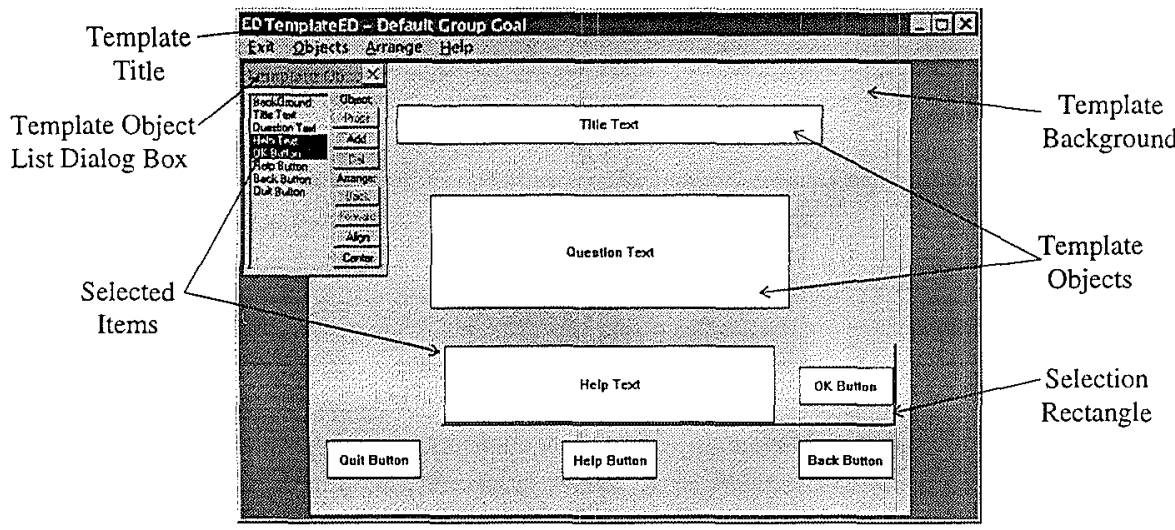

Figure 31: The Template Editor.

Objects may be selected in a number of different ways,

1. Clicking on an object in the Template View. Multiple objects are selected by clicking on objects whilst holding down either the Control or Shift Key.

2. Clicking on an empty part of the screen and dragging the selection rectangle around any number of template objects.

3. Selecting an object from the Template Object List. Multiple objects are selected by clicking on entries whilst holding down either the Control or Shift Key. This is useful for selecting objects that are obscured by other selected objects.

There are a number of operations that can be performed on template objects. These are available through the Template View menu and from the Template List dialog box.

- Add Object. Selecting this operation displays the 'New Object' dialog box, see Figure 32. Select from the list the object to be added to the template and press the 'OK' button. Now when the mouse cursor is over the Template View it will have the word 'Add' next to the point. Simply click on the Template View or drag out an area to place the new object. Note that only one animation object is allowed.

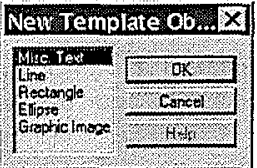

Figure 32: The New Template Object dialog box.

- Delete Object. A single object can be deleted from the template by selecting and the delete operation. Note that only objects added to the template with the Add Object operation can be deleted from the template. The default template objects can not be deleted.

- Arrange Forward. A single object can be moved forward in the template order with the operation. Moving the object forward will move it towards the bottom of the Template list. Note that an object can not be moved forward past the button objects as they sit on top of the screen by default.

- Arrange Back. A single object can be moved back in the template order with the operation. Moving the object back will move it towards the top of the Template list. Note that an object can not be moved back past the background as the background sits at the back of the screen by default.

- Align Objects. A group of objects can be aligned with respect to each other by using this command. Choosing this operation displays the Align Objects dialog box as in Figure 33. Object can be aligned horizontally and/or vertically by choosing the appropriate options. The options are,

1. No Change. Leaves the selected objects unchanged in that particular direction.

2. Left Sides/Tops. Aligns all the selected object's left/top edges to that of the leftmost/topmost object.

3. Centers. Aligns all the selected objects about the average position of all the objects.

4. Right Sides/Bottoms. Aligns all the selected object's right/bottom edges to that of the rightmost/bottom most object.

5. Space Equally. Spaces the selected objects according to the average amount of free height/width between the objects.

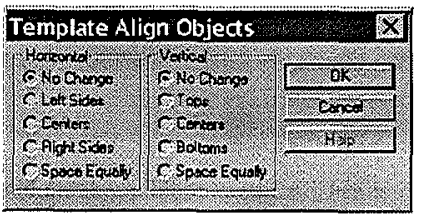

Figure 33: The Align Objects dialog box 
- Center Selection. A group of objects can be centered within the template either horizontally or vertically. A single object will be centered in the middle of the template. A group of objects will be centered as if they were a single object in the middle of the template.

- Move Selection. The current selected objects can be moved with either the keyboard or the mouse. Use the Cursor keys to move the selection up, down, left and right one unit at a time. To move the selection by larger steps, hold down the Shift key. Alternatively, click on the current selection with the mouse and drag the selection rectangle with the mouse. The current selection will be moved to the new position.

- Object Properties. The properties for a single object can be displayed by selecting the option as described above or by the object in the Template View or by double clicking it's entry in the Template List. This will display the object property dialog box for that object. The object properties are discussed in the next section.

To exit the template editor, select the 'Exit' menu item on the menu bar. The current template will be saved and the Tree Editor will appear.

\subsection{Object Properties}

There are four main types of template objects. These are Background, Button, Text and Graphic. Within these four main types there are a number of sub types. These are all discussed following,

- Background. This is the background for the template. It is a single colour rectangle that covers the whole screen. It is possible to have different colour backgrounds for different template. Figure 34 shows the Background object's template dialog box.

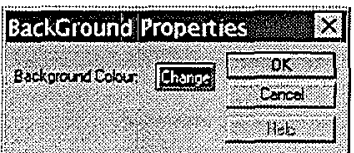

Figure 34: The Background template object's property dialog box.

- Button. There are four buttons used in each template. These are the 'OK' button, the 'Quit' button the 'Back' and the 'Help' button. Figure 35 shows the 'OK' button's property dialog box. Each of these buttons have the following properties,

1. Button Text. Can be used to change the text the button shows. For example the 'OK' button can be changed to 'Start' or 'Continue'.

2. Text Colour. The foreground colour of the button text. Press the associated 'Change' button to choose a different colour.
3. Button Font. The font used to draw the Button Text. Press the 'Change' button to choose a different fon

4. Face Colour. The colour of the button face.

5. Button Size. These fields show the size of the button in template units. Thus the absolute size and position of the button can be set from here.

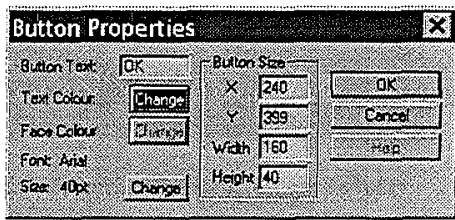

Figure 35: The Button object's properties dialog box

- Text. There are four main types of template text objects. 'Title' text, 'Question' text, 'Help' text and 'Miscellaneous' text. Figure 36 shows the text object's property dialog box. Each text object has the following properties,

1. Text Colour. The foreground colour of the text. Press the associated 'Change' button to choose a different colour.

2. Text Font. The font used to draw the Button Text. Press the 'Change' button to choose a different font

3. Text Alignment. Describes how the text will be formatted within its rectangle. Possible options are left, right, centered and justified.

4. Text Size. These fields show the size of the text object in template units. Thus the absolute size and position of the object can be set from here.

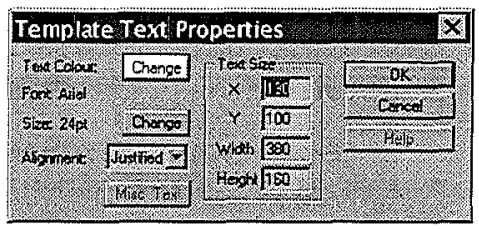

Figure 36: The Text object's properties dialog box

- Graphic. There are five different types of graphic. These are Rectangle, Ellipse, Graphic Image, Animation and Line. The first four graphic types use the graphic object property dialog box in Figure 37. Each of these four graphic objects have the following properties,

1. Fill Colour. The foreground colour of the object. Press the associated 'Change' button to choose a different colour. The graphic image and animation objects do not have this property.

2. Border Colour. The colour used to draw the edge of the object. The graphic image and animation objects do not have this property. 
3. Graphic Size. These fields show the size of the graphic object in template units. Thus the absolute size and position of the object can be set from here.

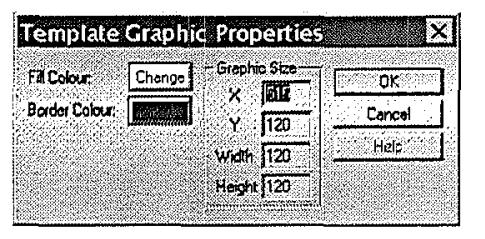

Figure 37: The Graphic object's property dialog box

The Line graphic has a different property dialog box as shown in Figure 38 . The line object behaves slightly differently to the other graphic objects as it can be horizontal or vertical. The Line Properties dialog box has the following controls,

1. Line Colour. The foreground colour of the object. Press the associated 'Change' button to choose a different colour.

2. Mirror Horizontal. Pressing this button flips the line around the vertical axis.

3. Mirror Vertical. Pressing this button flips the line around the horizontal axis.

4. Make Line Horizontal. Pressing this button makes the line horizontal. Once a line is horizontal it can not moved by the normal methods in the Template View. It can only be moved by adjusting the Graphic Size fields.

5. Make Line Vertical. Pressing this button makes the line vertical. Once a line is vertical it can not moved by the normal methods in the Template View. It can only be moved by adjusting the Graphic Size fields.

6. Graphic Size. These fields show the size of the line object in template units. Thus the absolute size and position of the object can be set from here.

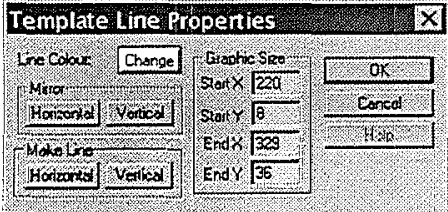

Figure 38: The Line object's property dialog box. 
D.2 QUESTEX USER GUIDE

D.2 QUESTEX USER GUIDE 



\section{The QUEST Questionnaire System}

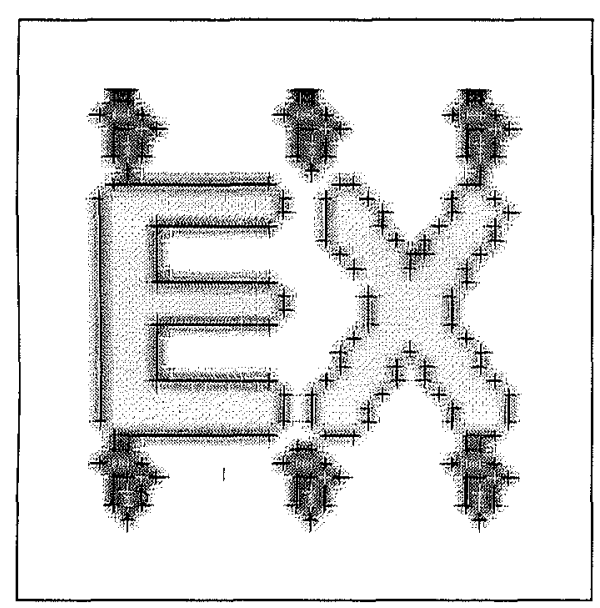

\section{QuestEX User Guide}

\section{Version 2.0}

Preliminary QuestEX User Guide.

This Users guide is divided into two parts. These are the Author's guide and the Respondent's guide. The author's guide explains the steps required to setup the QUEST system. The respondent's guide explains the steps involved in using QuestEX while answering a questionnaire.

1. Author's User Guide.

1.1 Installing QUEST.

QUEST is distributed via floppy disk. To install OUEST insert the floppy disk labeled 'QUEST - Disk \#1' into a floppy disk drive. Then run the setup program on the floppy disk, this can be done by,

1. Double clicking SETUP.EXE in file manager.

Start file manager, and change to the floppy disk drive that contains the QUEST files. Use the mouse to double click on the SETUP. EXE file item, or select the SETUP. EXE file item with the arrow keys and TAB and press ENTER.

2. Using the Run command in either file manager or program manager. Select the Run command from either file manager's or program manager's File menu. Type in the full path for the SETUP. EXE file and press the Enter key or the OK button.

Follow any prompts that are displayed during installation. 


\subsubsection{Running QuestEX.}

QuestEX can be started in a number of different ways

1. By double clicking on a program manager icon or shortcut

This is the recommended way of starting QuestEX. The icon or shortcut should have been created during installation.

2. By double clicking QUESTEX.EXE in File Manager or Explorer.

To start QuestEX this way, run file manager or Explorer and change to the directory containing QuestEX. Use the mouse to double click on the QUESTEX.EXE file item, or select the QUESTEX.EXE file item with the arrow keys and TAB and press ENTER.

3. By using the Run command.

Select the Run command from either lile manager's or program manager's File menu or the Start Menu. Type in the full path for the QUESTEX. EXE file and press the Enter key or the OK button.

4. By file association.

During installation a file association is setup for the extension .QS2 and the program as QUESTEX.EXE. This can be changed so that association is with QUESTEX. EXE. Then QuestEX can be started by double clicking on the QS2 data file in file manager. Note that an association can only be set-up for QuestEX or QuestED, not both.

5. With a command line argument.

When using the above methods 1 and 3 , a path and filename for a .QS2 questionnaire file can be specified after the QuestEX executable filename. This will automatically load the questionnaire file once QuestEX has started. For example, the following command line will start QuestEX and load the questionnaire file HEALTH. QS2.

$C: \backslash Q U E S T \backslash Q U E S T E X$. EXE $C: \backslash Q U E S T \backslash H E A L T H . Q S 2$

If QuestEX is started using a command line argument or by file association, the questionnaire file will be automatically loaded. QuestEX will then proceed straight to the questionnaire introduction. Otherwise a standard file open dialog box, as show in figure 1, will appear. Once a questionnaire has been selected, QuestEX will proceed to the questionnaire introduction. The 'Exit QuestEX' button allows you to exit QuestEX

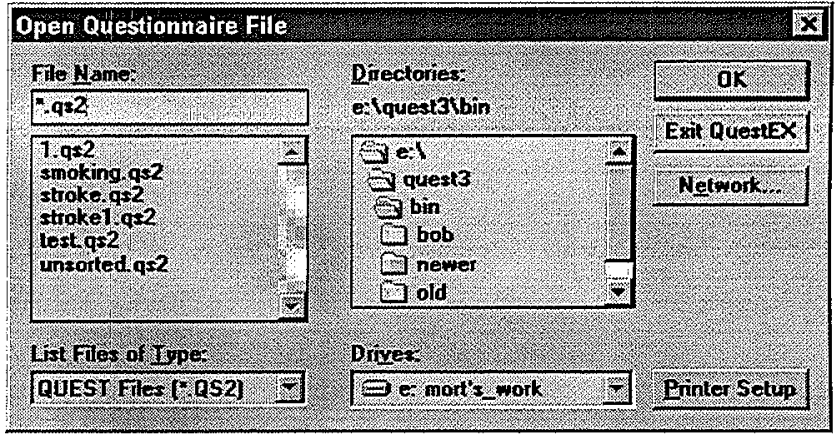

Figure 1: The open questionnaire file dialogue box

\subsubsection{Printer Setup.}

Pressing the 'Printer Setup' button allows you to choose the default printer for QuestEX. The standard printer setup dialog box, as shown in figure 2, will appear.

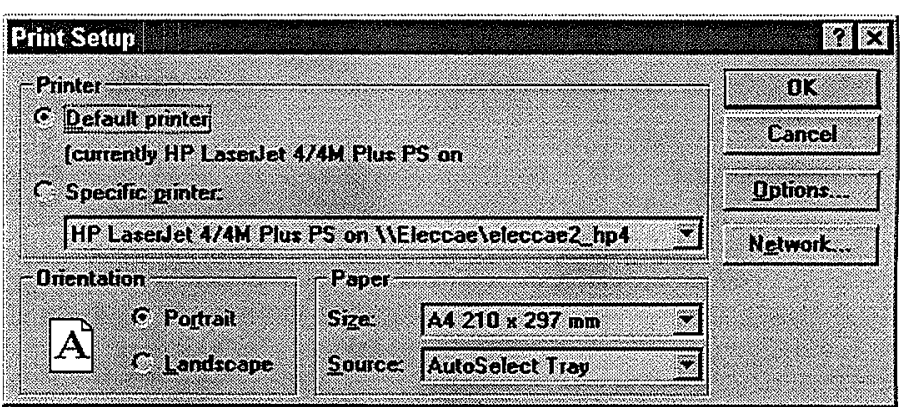

Figure 2: The open questionnaire file dialogue box.

From this dialog box choose the target printer from either the 'Default Printer' or from the list of 'Specific Printers'. Details such as paper orientation and size can also be setup here. For more detailed options press the 'Options...' button. The 'Network button can be used to setup printers on a Network. 


\section{Respondent's User Guide.}

\subsection{The Questionnaire Introduction.}

The first screen the respondent will see is the Questionnaire Introduction screen. This is the start of the questionnaire. The screen appearance of the Questionnaire Introduction is determined by the author of the questionnaire, however there are a number of items which will always appear on the introduction. These are,

- A Questionnaire Title. This just states the name of the questionnaire.

- A Questionnaire Description. The purpose of this description is to inform the respondent on what to expect in the questionnaire. It should 'sign-post' the questionnaire, indicating what will be asked, and in roughly what order

- The 'OK' Button. Pressing this button will start the questionnaire. This button may however have any name. For instance it may appear as the 'Continue' or 'Start' button. Pressing the 'Enter' key has the same effect as pressing the 'OK' button.

Then there are a number of items which may or may not appear. Some of these are,

- The 'Help' Button. Pressing this button will display some information about the questionnaire some-where on the Introduction screen. Pressing the 'Alt- $\mathrm{H}$ ' key combination has the same effect as pressing the 'Help' button.

- The 'Quit' Button. Pressing this button on a Questionnaire Introduction screen allows you to exit QUEST. If the Questionnaire has an Exit Password, then the dialog box in figure will appear, as in figure 3 . Enter the password and press the 'OK', QuestEX will close. If the Questionnaire does not have a password, then a yes/no prompt asking for confirmation will appear. Pressing 'Yes' will close QuestEX, otherwise you will return to the Questionnaire Introduction screen. Pressing the 'Alt-Q' key combination is the same as pressing the 'Quit' button.

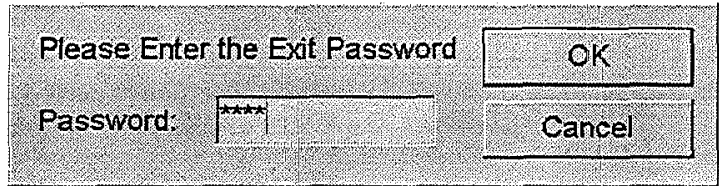

Figure 3: The Exit Password dialog box

- Graphics. Pictures just to enhance the introduction's appearance. Sound and Music may also accompany the introduction screen.

- Animation. A window that shows a video or graphic animation.
Once the 'OK button has been pressed, the questionnaire will start. Figure 4 shows a typical Questionnaire Introduction screen. Here the 'OK' Button has been labeled 'Start'.

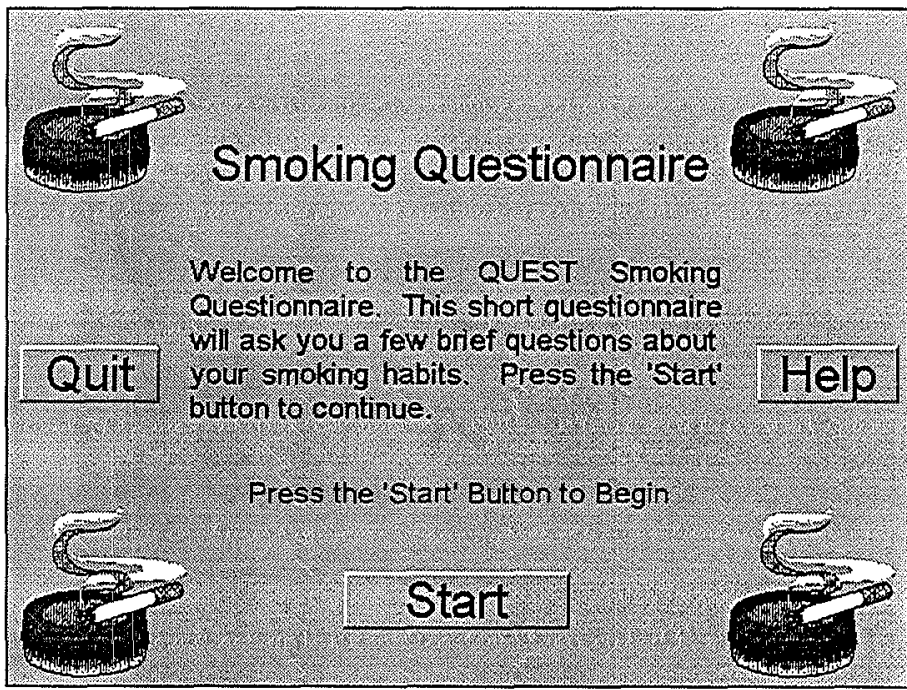

Figure 4: An example Questionnaire Introduction Screen.

\subsection{The Section Introductions.}

The next screen that appears is likely to be a Section Introduction, although this may not be the case. The Section Introductions appear very similar to the Questionnaire Introduction. Their purpose is to introduce each sub-section in the questionnaire. Again the same sort of items can appear in the Section Introduction as in the Questionnaire Introduction. There are also other items which may or may not appear these are,

- The 'Back' Button. Pressing this button will take you back to the last question you answered or the beginning of the questionnaire, which is closer. This allows you to re-enter your answer for that question. Pressing the 'Alt-B' key combination has the same effect as pressing the 'back' button

- The 'Quit' Button. Pressing this button on a section or question screen will initially prompt you for confirmation of the Quit action. If you answer 'Yes' then you will be taken back to the Questionnaire Introduction screen. If you answer 'No' then you will return to the current question or section. 


\subsection{The Questions.}

There are a number of different types of question that QUEST can ask. For each type, the respondent is prompted to reply in a different way. All the question types have number of common features. These are.

- The Question Text. This is the actual question the respondent is to answer.

- An 'OK' button. Pressing this button indicates that the respondent has entered their reply. If the reply has not be entered correctly, then a message is displayed indicating this, and the respondent is prompted to re-enter the reply. Pressing the 'Enter' key has the same effect as pressing the 'OK' button.

- A Reply Box. The reply box varies with the type of question. Each question type is given and the reply box is described.

1. Text Entry: The reply box is a text entry field. The respondent types their reply into this field. Any kind of text reply can be entered.

2. Numeric Entry: As for the Text Entry, the reply box is a text entry field However if the respondent does not enter a number, or if the number entered does not lie within a specific range the respondent is asked to re-enter their reply.

3. Single Selection List: The reply box is vertically scrolling list box. Within the list box is a set of pre-determined replies. One of these replies must be selected. The mouse can be used to select an item by positioning the cursor over the required item and pressing the left mouse button. The Arrow keys maybe used to move the selection bar up and down.

4. Multi-Selection List: Again the reply box is a vertically scrolling list box full of predetermined replies. However the respondent can now choose any number of the replies. This includes no replies. The Arrow keys maybe used to move the selection bar up and down. The Space bar can select and deselect each reply.

5. Information: There is no reply box for this type of question, in fact it isn't even a question. Its only purpose is to display information for the respondent.

If a 'Help' button appears in the question, pressing it will display some predetermined help information some where within the current screen. Also in certain situations this information maybe displayed after a set period of time.

The questionnaire is complete when all the questions have been asked. The Questionnaire Introduction screen is then displayed. Depending on the questionnaire, a summary of the respondent's replies can be printed out. 
D.3 QUESTED TUTORIAL

D.3 QUESTED TUTORIAL 


\section{The QUEST Questionnaire System}

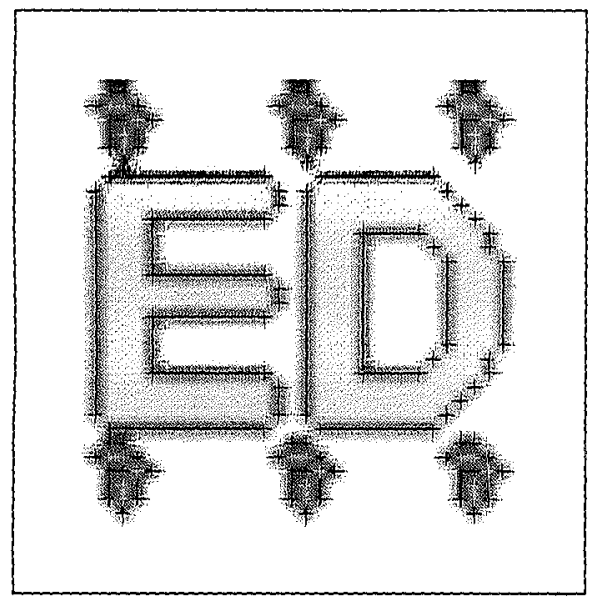

\section{QuestED Tutorial}

Version 2.0
Preliminary QuestED Tutorial.

This Tutorial presents the step by step construction of two simple questionnaires, 'Smoking' and 'Stroke'. These two small questionnaires are included in the Quest examples directory. Also in this directory are several other example questionnaire including 'Health check Staff survey', 'Audit' and the 'National Drug and Alcohol Research Readiness to Change' questionnaires. All these questionnaires demonstrate different aspects of Quest's capabilities.

This tutorial covers the questionnaire, summary and user interface aspects of the 'Smoking' and 'Stroke' questionnaires. For each questionnaire, the information requirements are examined and reduced in to questions. A step by step guide then shows how to enter the questionnaires into QuestED. The 'Stroke' questionnaire tutorial covers the generation of a simple summary, and simple changes to the questionnaire's user interface.

Finally there is an evaluation questionnaire which we would like you to complete. This guestionnaire will allow you to enter any suggestions and criticisms you have found in the Quest System. It will provide useful information and feedback for the developers on the current state of the project. For more information on this, read the developers on the current state of the project
'Readme.txt' file in the Quest directory.

By all means load and experiment with the example questions with both QuestED and QuestEX. This will give you a better feel for the system. 


\section{Smoking Questionnaire.}

This Smoking questionnaire originated from Dr. Keith Carey-Smith at the time of the General Practitioners conference in Christchurch. July 1995. It's original purpose was to test the new capabilities of Quest V2.0. The Smoking questionnaire is just a smal part of what would be a larger questionnaire, but it demonstrates the concepts well. The main aim of the questionnaire is to learn if the questionnaire respondent is possibly addicted to nicotine.

The best way to approach this problem is to break the problem into progressively smaller and smaller picees. This hierarchical approach is very similar to how Quest represents a questionnaire. Quest breaks a questionnaire into 'Goals'. Questions, Calculations and Conditions are goals. Note that these goals all gather information of some sort from the respondent. Group and Info goals however give information to the some sort from

The top level of the problem is to determine the answer to 'Is the patient possibly addicted to nicotine?" The answer is yes if they display the right symptoms. This situation is represented in Figure 1.

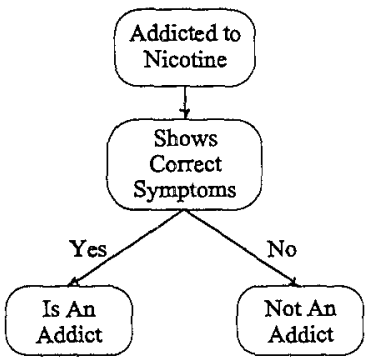

Figure 1: Step I. The simplest level of the smoking questionnair

So what are the correct symptoms? The patient is possibly an addict if

- they crave cigarettes when without.

- or they feel irritable if they haven't smoked for a while.

- or they usually smoke with in thirty minutes of waking.

This second step is shown in Figure 2.

Of course asking these questions to every patient is inefficient. We can do better by focusing on patients who have a greater risk of being an addict. Firstly the patien must at least smoke to be considered at risk. Secondly a heavy smoker has an increased risk.

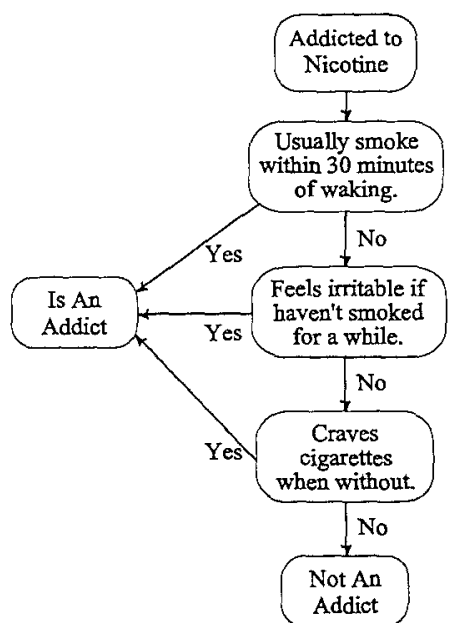

Figure 2: Step 2. Including the detailed symptoms.

By eliminating non-smokers and light-smokers from the equation, we can make the questionnaire more efficient and effective. This latest step is shown in Figure 3. To determine if the patient is a heavy smoker we ask them how many cigarettes they smoke per day. If it is over a certain amount them we can consider them at risk.

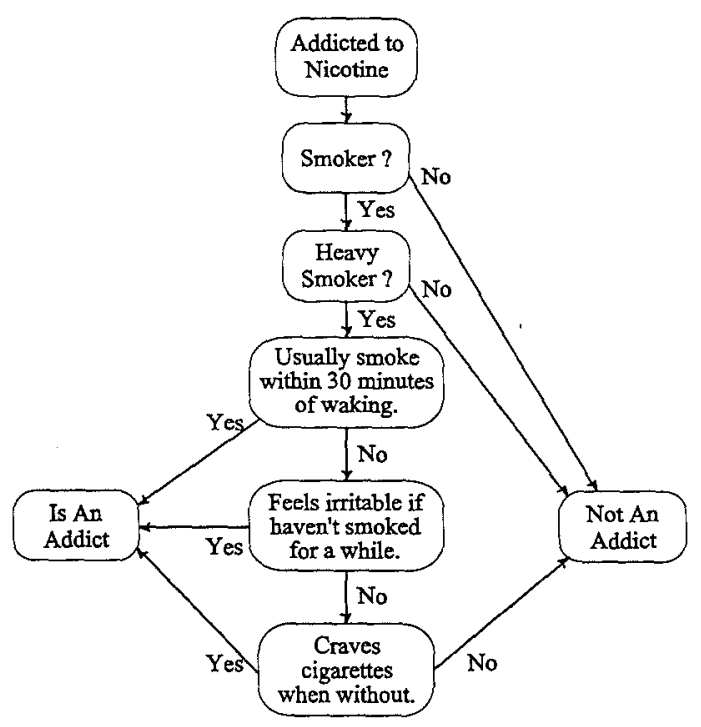

Figure 3: Step 3. Eliminating redundant questions with further condition 
The steps required to enter this questionnaire into QuestED are as follows. If you make a mistake and have to delete questions, use the QuestionnairelRenumber Questionnaire menu command to renumber the questionnaire. A phrase that appears in bold is either a menu command, the name of a dialog box. property panel control or fields or an option that appears within QuestED. A phrase with a box around it is a reference to a goal in the questionnaire tree view.

1. Use the FilelNew menu command to create a new questionnaire. Enter some questionnaire introduction text into the Goal Information: Description field on the property panel. Also enter a title into the Goal Information: Title field.

2. Create a Single Select sub goal of Group 1 by pressing the Sub Goals: Add button. From the Add Sub Goal dialog box, choose the A New Goal option and then the SingleSel List item from the drop down list. Press OK. Select the new SList 2 goal. Enter the question "Do you smoke" in the Goal Information: Question Text and replies 'Yes' and 'No' using the Reply Field: Add button.

3. Select the Group 1 goal, and create the Group 3 sub goal as done in step 2 except select the Group Goal item from the drop down list. As the Group 3 goal will be hidden, select it, press the User Interface: Select button and select None from the Select Template dialog box, press 'OK'.

4. The Group 3 goal is conditional on the answer of Goal 2, so press the Condition Goal: Add button. The condition goal Cond 4 will appear.

5. Select the Cond 4 goal. To link the SList 2 goal to the Cond 4 goal, press the Sub Goals: Add button. From the Add Sub Goal dialog, select the Existing Goal option and choose the SList 2 item and press 'OK'. SList 2 will appear as a referenced child goal of Cond 4. Your questionnaire should look like Figure 4.

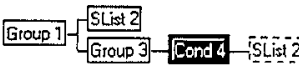

Figure 4: The smoking questionnaire after 5 steps.

6. To create the Goal 4's condition, select the SList 2 item from the Sub Goals: list. Press the Insert into: LHS button. From the Insert Reference dialog box choose the List Index option and press OK. Select the ' $=$ ' sign in the Relation field and enter ' 1 ' into the RHS field.

7. Now select Group 3, and create SList 5 as in step 2. Select the SList 5 goal and enter the question, "How many cigarettes do you smoke per day?" and the responses 'Between 1 and 5', 'Between 5 and 10', 'Between 10 and 20' and 'Above 20'.

8. Select Group 3 and create Group Goal 6 as in step 3. As the Group 6 goal will be hidden, select it and press the User Interface: Select button and select None.
9. Group Goal 6 is conditional on the answer of Goal 5, so press the Condition Goal: Add button. Condition Goal 7 will appear.

10. Select the Cond 7 goal. To link Goal 5 to Goal 7 , press the Sub Goals: Add button. From the Add Sub Goal dialog, select the Existing Goal option and choose the SList 5 item. STist 5 will appear as a referenced child goal of Goal 7 Your questionnaire should look like Figure 5 .

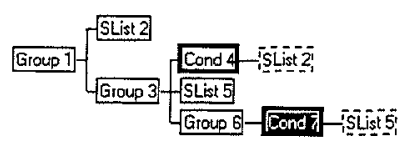

Figure 5: The smoking questionnaire after 10 steps.

11. To create the Goal 7's condition, select the SList 5 item from the Sub Goals: list. Press the Insert into: LHS button. From the Insert Reference dialog box choose the List Index option and press OK. Select the '>=' sign in the Relation field and enter ' 3 ' into the RHS field. This condition will be true if one of the last two responses in Goal 5 were selected by the patient.

12. Select the Group 6 goal and create three Single Select Goals 8,9 and 10 as in step 2. Select SList 8 and enter the question, "Do you feel irritable if you haven't smoked for a while?' and the replies 'Yes' and 'No'. Enable the Reply Field: Use Values check box. A ' 0 ' will appear before each of the responses. Double click on the 'Yes' reply. In the Edit Reply Item with Value dialog box, change click on the 'Yes' reply. In the
the ' 0 ' value to ' 1 ' and press $\mathrm{OK}$.

13. Press the Reply Field: Copy button. The Copy Replies dialog box will appear with a list containing all of the Single Select questions. From the list select SList 9 and SList 10 and press $O K$

14. Select Goals 9 and 10 and enter the questions "Do you usually smoke with in thirty minutes of waking?" and "Do you crave for a cigarette when without them?" respectively. Enable the Reply Field: Use Values option for both goals. Note that the 'Yes' and 'No' replies have been copied from SList 8.

15. To display a warning to the patient, select Group 6 and create Info Goal 11 as in step 2. Your questionnaire should look like Figure 6.

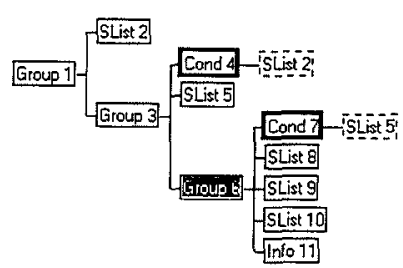

Figure 6: the smoking questionnaire after 15 steps. 
16. Info Goal 11 is conditional on the symptoms, so select it and press the Condition Goal: Add button. The Cond 1p goal will appear. Select Cond 12 and create Calculation Goal 13.

17. To create the Goal 12's condition, select the Calc $\mathbf{1 3}$ item from the Sub Goals: list. Press the Insert into: LHS button. From the Insert Reference dialog box choose the Numeric variable option and press $O K$. Select the ' $>$ ' sign in the Relation field and enter ' 0 ' into the RHS field.

18. Select the Calc 13 goal and create references to Goals 8,9 and 10 by using the Sub Goals: Add button as in step 5 .

19. Enter the calculation " $g(8, v)+g(9, v)+g(10, v)$ " either by hand or by selecting item from the Sub Goals: List and pressing the Insert Into Calc button. Do not press enter at the end of the calculation. The result of this calculation will be press enter at the end of the calculation. The result of this calculation
greater than zero if the patient answers 'Yes' to any of Goals 8,9 and 10.

20. Select the Info 11 Goal, and create a reference to Calc 13. Now enter "You answered $g(13, v)$ out of the last three questions positively. You are likely to be to addicted to nicotine and need help to stop." in to the Goal Information: Information Text field. The goal reference ' $g(13, v)^{\prime}$ can be entered using the Insert in Text button. Your questionnaire should look like Figure 7.

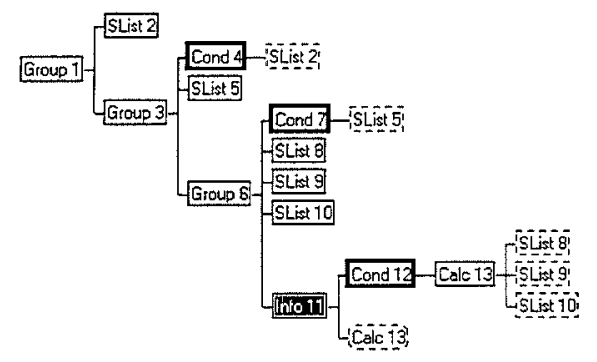

Figure 7: The smoking questionnaire after 20 step

Congratulations you have now entered the basic questionnaire structure for the Smoking questionnaire. Save your yersion of the questionnaire and run the questionnaire with QuestEX. Now load the Smoking questionnaire from the example questionnaire with QuestEX. Now load the Smoking questionnaire from the example These goals take the chance to ask the patient about their motivation to quit smoking, and whether they want more information about the effects of smoking. Examining them is left as a further exercise.

\section{Stroke Questionnaire}

The stroke questionnaire was derived from a journal article which outlined a research study into high risk stroke patients. The research, published in the British Journal of General Practice reports that the most high risk stroke patients could be identified using a simple scoring system. Using age, systolic blood pressure, current cigarette consumption and evidence of anginal chest pain, researches were able to predict 82 per cent of all strokes over a five year period in the high risk group.

One example scoring system is,

- Multiply the patient's age by nine.

- Add 2.85 times the patient's systolic blood pressure.

- Add 70 if angina is present.

- Add 90 if the patient smokes between one an twenty cigarettes per day

- Add 130 if the patient smokes more than 20 cigarettes per day.

- Target patients scoring over 1001 .

For a simple implementation of this scoring system, we need to do the following things,

1. Get the information from the patient for the calculation,

2. Calculation the score,

3. Make a decision based on the target threshold,

4. Display the results of the decision,

5. Generate a hard copy summary of the results.

The steps required to enter this questionnaire into QuestED are as follows,

1. Use the FilelNew menu command to create a new questionnaire. Enter some questionnaire introduction text into the Goal Information: Description field on the property panel. Also enter a title into the Goal Information: Title field.

2. Create the Info 2 goal of Goal by pressing the Sub Goals: Add button. This goal will display a warning to the patient if their score is greater than 1000. Select Inio 2 and create the Cond 3 goal with the Condition Goal: Add button. Select Cond 3.

3. Create the Calc 4 sub goal of Cond 3 by pressing the Sub Goals: Add button. To create the Cond 3's condition, select the Calc 4 item from the Sub Goals: list. Press the Insert into: LHS button. From the Insert Reference dialog box choose the Numeric variable option and press OK. Select the ' $>=$ ' sign in the Relation field and enter ' 1000 ' into the RHS field.

4. Select the Into goal and create a reference to the Calc 4 goal by pressing the Sub Goals: Add button. From the Add Sub Goal dialog, select the Existing Goal option and choose the Calc 4 item. Calc 4 will appear as a referenced child goal of Info 2. 
5. Enter the following information text into the Info 2 goal, "Your risk factor is $\mathrm{g}(4, \mathrm{v})$. This is greater than 1001. You maybe at risk from stroke." and the title "Results - Warning Possible Risk".

6. Select the Calc 4 goal, and create two Numeric Entry Goals 5 and 6 . Then Create two Single Select Goals 7 and 8 . Your questionnaire should look like Figure 8.

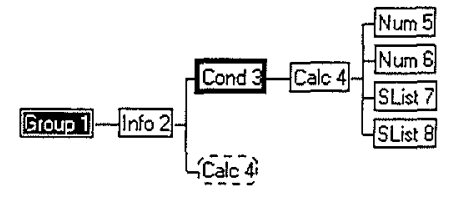

Figure 8: The stroke questionnuire uffer 6 steps.

8. Select the Num 5 goal and enter the question "What is your Age?". Enable the 'Limit' check option and enter 0 and 120 as the lower and upper limits.

9. Select the Num 6 goal and enter the question "What is your systolic blood pressure?". Again enable the 'Limit' check option and enter sensible limit values.

10. Select the SList 7 goal and enter the question "Do you have angina?" and the responses 'Yes' and 'No'. Check the 'Use Values' option and change the value for 'Yes' from 0 to 1 .

11. Select SList g goal and enter the question "How many cigarettes do you smoke per day?" and the responses 'None', 'Between 1 and 20' and 'Over 20'. Check the 'Use Values' option and change the value for 'Between 1 and 20 ' to 90 and the value for 'Over 20 ' to 130 .

12. Select the Calc 4 goal and enter the calculation " $9 * \mathrm{~g}(7, \mathrm{v})+2.85 * \mathrm{~g}(6, \mathrm{v})+70^{*} \mathrm{~g}(5, \mathrm{v})$ $+g(8, v)$ " either by hand or by selecting a item from the Sub Goals: List and pressing the Insert Into Calc. Do not press enter at the end of the calculation.

13. Select the Group goal and create Information Goal 9. This Info Goal will be conditional so select it and create Condition Goal 10 with the Condition Goal: Add. Select Cond 10

14. Link Calc 4 to Cond 10 with the Sub Goals: Add button, as in step 4 .

15. To create the Cond 10 's condition, select the Calc 4 item from the Sub Goal: list. Press the Insert into: LHS button. From the Insert Reference dialog box choose the Numeric variable option and press OK. Select the ' $<=$ ' sign in the Relation field and enter ' 1000 ' into the RHS field.

16. Select the Info 9 goal and create a reference to Calc 4 as in step 4, Calc 4: will appear as a referenced child goal of Info 9 .
17. Enter the following information text into Info 9 , "Your risk factor is $g(4, v)$. This is not greater than 1001 , so you are not currently considered at risk.". Also enter the title, "Results - Patient Not At Risk".

You have now created the stroke questionnaire. It should appear as in Figure 9.

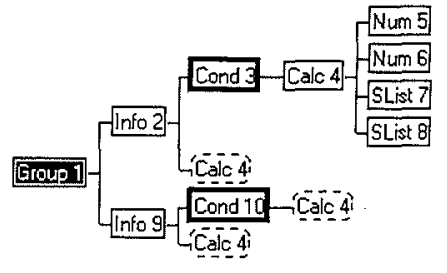

Figure 9: The correct appearance of the Stroke Questionnaire.

Next to create a simple summary for the stroke questionnaire follow these steps,

1. Choose the SummarylView Summaries menu option. The View Summaries dialog box will appear, press the Add button. Press the 'OK' button the Summary Style dialog box.

2. In the Summary Edit dialog box, enter your summary name into Name: field.

3. Select all of the items in the Questionnaire List except for the two condition items. Press the Add $\rightarrow$ Button. These items will be transferred to the Summary List.

4. Use the 'Up' and 'Down' buttons to re-order the items in the Summary List. Make the order; $1,5,6,7,8,4,9$ and 2 .

5. For each of the items in the Summary List double click on it and change the default text to suit. Make sure you leave any ' $\wedge$ ' symbols in each entry's text. Use the delimiter option to format and group the summary entries. For example use the 'Blank Line' delimiter for Goals 1 and 4, and ' $1 / 2$ Page Tab' for Goals 5 and

The example Stroke Questionnaire from the example directory produces the following summary output, 


\section{Patient Stroke Evaluation}

$\begin{array}{ll}\text { Patient Age?: } 32 & \text { Blood pressure: } 169 \\ \text { Angina?: Yes } & \text { Smokes per day? : Be }\end{array}$

Risk $=929.65$

Smokes per day?: Between 1 and 20

Patient is not at Risk

To make some simple user interface changes to the stroke questionnaire, select the Group 1 goal. Then press the User Interface: Edit button. The main window changes to show the screen layout for the Default Group Goal Template. This is the template the Group 1 goal is using. To change the colour and font of the template title,

1. Select the rectangle with the label 'Title Text'.

2. Press the Props button on the Template Object dialog box. Alternatively you can double click on the rectangle itself. The Template Text Properties dialog box will appear.

3. Use the two Change buttons to alter the colour, font and font size of the title text

4. The Alignment of the title text can also be changed to either left, centered, right or justified.

5. Select the Exit menu option to return to the questionnaire editor

6. To see the changes you have made to the template, select the User Interface: Test button. This will show you the goal exactly as it will appear in QuestEX. Press the OK button to return to the questionnaire.

The properties of any object in the Template Editor display can be shown by double clicking on the selected object

Finally make sure you save and run each of the tutorial questionnaires with QuestEX. 